Dagmar Abendroth-Timmer / Eva-Maria Hennig (eds.)

\title{
Plurilingualism and Multiliteracies
}

International Research on Identity Construction in Language Education 


\section{Dagmar Abendroth-Timmer / Eva-Maria Hennig (eds.)}

\section{Plurilingualism and Multiliteracies}

LANGSCAPE is a plurilingual and multicultural international research network on language acquisition and language education. The current research focus is on Identity Construction in Language Education. This volume summarizes some research results of the last four years by presenting empirical research projects as well as theoretical concepts. The contributions all deal with topics linked to plurilingualism or to certain aspects of the concept of multiliteracies like globalization, language policy, multiculturalism, multimodal communication processes, intercultural learning etc. The authors conceptualize or analyse identity construction processes of learners and educators in different plurilingual and multicultural learning environments or media based settings.

\section{The Editors}

Dagmar Abendroth-Timmer: professor at the University of Siegen (Didactics of French and Spanish languages and cultures). Research interests: multiliteracies, multiculturalism, intercultural learning, CLIL, motivation, teacher education.

Eva-Maria Hennig: research assistant and $\mathrm{PhD}$ student at the University of Siegen (Didactics of French and Spanish languages and cultures). Research interests: multiliteracies, didactics of literature/film/ media, inter- and transcultural learning, emotions in foreign language learning. 
Plurilingualism and Multiliteracies 


\section{KOLLOQUIUM FREMDSPRACHENUNTERRICHT \\ Herausgegeben von Daniela Caspari, Lars Schmelter, Karin Vogt und Nicola Würfel}

BAND 50

Zu Qualitätssicherung und Peer Review der vorliegenden Publikation:

Die Qualität der in dieser Reihe erscheinenden Arbeiten wird vor der Publikation durch alle vier Herausgeber der Reihe geprüft.
Notes on the quality assurance and peer review of this publication:

Prior to publication, the quality of the work published in this series is reviewed by all four editors of the series. 


\section{Dagmar Abendroth-Timmer / Eva-Maria Hennig (eds.)}

\section{Plurilingualism and Multiliteracies}

International Research on Identity Construction in Language Education 


\section{Bibliographic Information published by the Deutsche Nationalbibliothek}

The Deutsche Nationalbibliothek lists this publication in the Deutsche Nationalbibliografie; detailed bibliographic data is available in the internet at http://dnb.d-nb.de.

\section{Library of Congress Cataloging-in-Publication Data}

Plurilingualism and multiliteracies : international research on identity construction in language education / Dagmar Abendroth-Timmer, Eva-Maria Hennig (eds.).

pages $\mathrm{cm}$. - (Kolloquium Fremdsprachenunterricht ; Band 50) Articles in English, French, and German. ISBN 978-3-631-62926-0

1. Language acquisition-Study and teaching. 2. Language and languagesStudy and teaching. 3. Language and education. 4. Multilingualism.

5. Literacy. I. Abendroth-Timmer, Dagmar, editor of compilation.

II. Hennig, Eva-Maria, 1983- editor of compilation.

P118.15.P58 2013

$306.44^{\prime} 6-\mathrm{dc} 23$

2013019869

Cover logo:

Christoph Baum

ISSN 1437-7829

ISBN 978-3-631-62926-0 (Print)

E-ISBN 978-3-653-03229-1 (E-Book)

DOI 10.3726/978-3-653-03229-1

(c) Peter Lang $\mathrm{GmbH}$

Internationaler Verlag der Wissenschaften

Frankfurt am Main 2014

Peter Lang Edition is an Imprint of Peter Lang GmbH

Peter Lang - Frankfurt am Main · Bern · Bruxelles .

New York · Oxford $\cdot$ Warszawa $\cdot$ Wien

This book is an open access book and available on www.oapen.org and www.peterlang.com.

This work is licensed under the Creative Commons

Attribution-NonCommercial-NoDerivs 4.0 which means that the text may be used for non-commercial purposes, provided credit is given to the author. For details go to http://creativecommons.org/licenses/by-nc-nd/4.0/

\section{(cc) BY-NC-ND}

This book is part of the Peter Lang Edition list and was peer reviewed prior to publication. 


\section{Content}

\section{Preface}

Dagmar Abendroth-Timmer / Gerhard Bach / Eva-Maria Hennig

LANGSCAPE - Networking the International Research Community on

Language Acquisition and Language Learning................................9

\section{Introduction}

Dagmar Abendroth-Timmer / Eva-Maria Hennig

Plurilingualism and Multiliteracies: Identity Construction in Language

Education.

\section{Contexts of Mobility and Ecology of Multilingualism}

Anne Pauwels

Rethinking the Learning of Languages in the Context of Globalization and

Hyperlingualism

Christiane Fäcke

Le plurilinguisme est-il un objectif européen pour l'Allemagne?

Martha C. Pennington / Itesh Sachdev / Lawrence Lau (†)

Language Use by London Bangladeshi and Chinese Adolescents:

Some Language Diary Data.

Sabine Ehrhart

Le développement plurilingue et interculturel en milieu éducatif ouvert

à la diversité - étude et bilan de trois projets universitaires avec la

participation d'une écologie linguistique « à la luxembourgeoise »

Claus Gnutzmann / Jenny Jakisch / Frank Rabe

Englisch als europäische Lingua franca.

Identitätsentwürfe, Erfahrungen und Einstellungen von Studierenden

\section{Multiliteracies and the Construction of Plurilingual Identities in Foreign Language Learning and Teaching}

Stephan Breidbach / Lutz Küster

'Bildung', 'Multiliteracies' and 'Identity' - Key Concepts in Language

Education in the Light of Sociocultural Theory and Dynamic Systems

Theory 
Martina Weyreter / Britta Viebrock

Identity Construction in Adult Learners of English for Specific

Purposes (ESP): Exploring a Complex Phenomenon........................145

Alice Chik

Constructing German Learner Identities

in Online and Offline environments.

Daniela Elsner

Multilingual Virtual Talking Books (MuViT) - A Project to Foster

Multilingualism, Language Awareness, and Media Competency.............. 175

Eva De Mesmaeker / Katja Lochtman

Belgian CLIL Teachers' Professional Identity

\section{Teacher Education and the Development of Multiliteracies}

Jean-Paul Narcy-Combes / Marie-Françoise Narcy-Combes

Formations hybrides en milieu pluriculturel:

comment concilier théories, pratiques et contraintes.

Anja Wildemann / Mahzad Hoodgarzadeh / Olga Esteve / Rebecca Walter

Ein Beitrag zur Sensibilisierung für eine Mehrsprachigkeitsdidaktik in der Lehrerbildung.

Claudia Frevel

Zur Ausbildung und Entwicklung adaptiver Lehrkompetenz angehender

Fremdsprachenlehrerinnen und -lehrer durch Videofeedback.... .243

Özlem Etuş / Katrin Schultze

English as a Lingua Franca and the Envisioning of Pre-service

English Language Teacher Education in the 21st Century:

Narrative Insights from İstanbul and Berlin

Mark Bechtel / Maud Ciekanski

Comment retracer les compétences interculturelles en interaction

dans la formation en ligne des enseignants de langues?

Une réflexion méthodologique.

Dagmar Abendroth-Timmer / Jose I. Aguilar Rio

Accompagner la formation de futurs enseignants de langue en tandem interculturel médiatisé: la sensibilisation aux fonctions du tutorat.

Authors 


\section{Preface}





\title{
LANGSCAPE - Internationale Forschergruppe zur sprachlichen Bildung
}

\author{
Dagmar Abendroth-Timmer / Gerhard Bach / Eva-Maria Hennig
}

LANGSCAPE begann die Forschungsaktivitäten zum Spracherwerb und Sprachenlernen mit einer Kerngruppe europäischer Partner im Jahre 1998 und wurde seither stetig erweitert. LANGSCAPE ist eine internationale Forschergruppe mit einer offenen, netzwerkeigenen Struktur. Das Netzwerk entsprang dem Forscherwillen von Gisèle Holtzer, Gerhard Bach und Michael Wendt und wurde von vielen Kolleginnen und Kollegen vor Ort und auf internationaler Ebene mitgeprägt und ausgestaltet. 2009 übernahm Dagmar Abendroth-Timmer die Funktion der Koordinatorin, unterstützt von dem ehemaligen Koordinator Gerhard Bach als wissenschaftlichem Berater. Der LANGSCAPE Koordinator trägt die Verantwortung, Partnern und Interessierten Informationen zugänglich zu machen, Mitglieder zu vernetzen, aktuelle Forschungsprojekte anzuregen und gemeinsame Tagungen zu unterstützen. Dies wird wiederum unterstützt durch das LANGSCAPE-Office, welches die Weiterleitung von Informationen im Forschungsnetzwerk und die Organisation von Tagungen gewährleistet. Dieses wurde von 2006 bis 2009 von Lena Bellingrodt (Bremen) und von 2009 bis 2013 von Eva-Maria Hennig (Siegen) geführt. Damit wurden jeweils Promotionsstellen geschaffen und von den Universitäten Bremen und Siegen finanziert. Die hohe Qualität aller LANGSCAPE-Forschungsaktivitäten wird vom wissenschaftlichen Berater unterstützt. Eine repräsentative Zahl an Partnern bringt im internationalen Vorstand ihre Expertise in die LANGSCAPE-Aktivitäten ein. Von 2013 an wird LANGSCAPE von Berlin aus unter der Leitung von Stephan Breidbach und Lutz Küster fortgeführt.

Seit 1999 hat LANGSCAPE 14 internationale Tagungen abgehalten. Die jeweiligen Beiträge wurden von internationalen Herausgebergruppen in mehrsprachigen Sammelbänden in der Reihe Kolloquium Fremdsprachenunterricht (KFU) publiziert und sind bei Peter Lang erschienen. Viele weitere Kongresse wurden vor Ort von LANGSCAPE-Mitgliedern organisiert. 2009 wurden Expertentreffen eingeführt, um Kerngruppen zu bilden und über die weitere Ausrichtung von LANGSCAPE zu entscheiden. Mit dem Ziel, andere Forschungskontexte, Bildungssysteme und Universitäten kennenzulernen sowie gemeinsam Doktorandinnen und Doktoranden in binationalen Promotionsverfahren (co-tutelle) $\mathrm{zu}$ betreuen, wurden Erasmus-Mobilitäten genutzt. Hierdurch ermöglicht LANGSCAPE den Austausch von Expertenwissen und die ertragreiche Weitergabe von Forschungsergebnissen und von Praxisbeispielen, die Modellcharakter haben (samples of good practice). Die LANGSCAPE- 
Aktivitäten geben Nachwuchswissenschaftlerinnen und -wissenschaftlern die Möglichkeit, andere Forschungsansätze kennen zu lernen, ihre Forschungsmethodik zu hinterfragen und auf einer internationalen Ebene integriert $\mathrm{zu}$ werden. Bei dieser gemeinsamen Arbeit verfolgt die LANGSCAPE-Forschergruppe folgende Prinzipien:

- Mehrsprachigkeit: LANGSCAPE versteht sich als mehrsprachige Forschergemeinschaft. Für die gemeinsame Forschung und die transnationalen Doktorandenprogramme wurden daher Grundprinzipien der Mehrsprachigkeit festgelegt und Strategien entwickelt, die sprachlicher Abgrenzung entgegenwirken sollen.

- Vielfalt und Vernetzung: LANGSCAPE bringt Partner aus aller Welt mit dem Ziel zusammen, unterschiedliche Forschungsansätze zu vergleichen und diese dann auch in anderen Kontexten einzusetzen.

- Zugang und Transfer: LANGSCAPE macht internationales Fachwissen auf lokaler Ebene für Forschung, Bildung und Fortbildung zugänglich.

- Kompetenz: LANGSCAPE stärkt den Zusammenschluss der Mitglieder für Forschungsprojekte auf nationaler und internationaler Ebene.

Die Forscherinnen und Forscher wollen sprachlicher, kultureller und methodischer Abgrenzung in der Fremdsprachenforschung entgegenwirken, indem sie Mehrsprachigkeit, Vielfalt und Multiliteralität stärken: Im Sinne sprachlicher und kultureller Handlungskompetenz und Integration schenken sie der sozialen Bedeutung von Mobilität, Migration, Medien und Gender besondere Beachtung. Dem liegt die Vorstellung einer Forschung zugrunde, die fachspezifische und geographische Grenzen überschreitet. Auf Basis der Annahme, dass sprachliche Fähigkeiten die zentrale Grundlage der Identitätsbildung darstellen, beschäftigen sich die LANGSCAPE-Forscherinnen und -Forscher eingehend mit dem Zusammenhang zwischen sprachlichen Fähigkeiten und der Konstruktion von sozialer Identität und Mehrsprachigkeit.

Daher ist der derzeitige Hauptfokus der Forschergruppe die Erfassung von Prozessen der Identitätskonstruktion in der sprachlichen Bildung. Innerhalb dieses Forschungsbereichs unterscheidet LANGSCAPE drei Säulen:

- Kontexte von Mobilität und Sprachenpolitik

- Institutionelles Lehren und Lernen von Sprachen

- Lehrerbildung und -professionalität

Um Forschungsergebnisse zu diesen Themenkomplexen auszutauschen und Forscherinnen und Forscher zusammenzubringen, die auf verwandten Gebieten forschen, wurde eine Reihe von Expertentreffen veranstaltet. Die Artikel dieses Sammelbandes spiegeln das Ergebnis dieses intensiven und fortwährenden Forschungsprozesses wider, der zum Zusammenschluss kleinerer internationaler 
Forscherteams führte; zu nennen sind das von Comenius geförderte MuVit-Projekt (Deutschland, Spanien, Türkei) oder das deutsch-französische INFRALProjekt, das über den DAAD/PROCOPE finanziert wurde, das blendedlearning-Projekt mit Lehramtsstudierenden aus Paris und Siegen, und zwischenzeitlich auch aus Berlin, und das größere Projekt Existing in languages von Partnern aus China, Frankreich, Deutschland und der Türkei.

Eine Vielzahl an lokalen Projekten und Einzelprojekten vervollständigen die Forschung zu Mehrsprachigkeit, Multiliteralität und Identitätskonstruktion beim Lehren und Lernen von Sprachen und werden in diesem Sammelband vorgestellt.

\section{LANGSCAPE - Networking the International Research Community on Language Acquisition and Language Learning}

\section{Dagmar Abendroth-Timmer / Gerhard Bach / Eva-Maria Hennig}

LANGSCAPE started research activities on language acquisition and language learning with a core group of European partners in 1998 and, over the last decade and a half, has expanded steadily. LANGSCAPE is an open-structured and international research group. It arose from the research interests of Gisèle Holtzer, Gerhard Bach and Michael Wendt, and was inspired by their local and international colleagues. In 2009, Dagmar Abendroth-Timmer took over the function of coordinator from Gerhard Bach, who now assists the network as research consultant. The LANGSCAPE coordinator takes responsibility for disseminating information on LANGSCAPE activities, networking, developing current research projects and enhancing common conferences. These functions are managed by the LANGSCAPE office which ensures the distribution of information within the research network and the organization of conferences. The office was managed by Lena Bellingrodt (Bremen) from 2006 to 2009, and from 2009 to 2013 by Eva-Maria Hennig (Siegen). In this way, two positions for $\mathrm{PhD}$-students were created and financed by the Universities of Bremen and, respectively, Siegen. The high quality of all LANGSCAPE research activities is supervised by the research consultant. A representative number of partners serving on the international board of directors account for all LANGSCAPE activities. In 2013, LANGSCAPE will move from the University of Siegen to the Humboldt University of Berlin, then coordinated by Stephan Breidbach and Lutz Küster. 
Since 1999, 14 international conferences were organized by LANGSCAPE partners whose proceedings were published in succeeding volumes in the network's multilingual series Kolloquium Fremdsprachenunterricht (KFU, Peter Lang Publishers), many of these in plurilingual form edited by international editorial groups. Several further conferences were conducted by LANGSCAPE members at the local level. In addition, expert meetings have been held from 2009 onwards with the purpose of networking major research partners and groups and with the focus on LANGSCAPE's future developments. The Erasmus mobility program was utilized to expand and differentiate diverse research contexts, to learn about different educational systems and university programs, and in particular to supervise $\mathrm{PhD}$-students in a joint $\mathrm{PhD}$-program (co-tutelle). Across the entire range of its activities, LANGSCAPE allows for the exchange of expertise and the profitable dissemination of research results and samples of good practice. The LANGSCAPE activities give junior researchers the opportunity to become acquainted with different research approaches, to investigate and query their own methods and approaches, and to network on an international level. In all of this, LANGSCAPE researchers pursue the following principles:

- Plurilingualism: LANGSCAPE itself operates as a plurilingual research community. It uses this expertise to develop effective strategies to counteract linguistic fragmentation by implementing its principles of plurilingual research and transnational doctoral programs.

- Networking Diversity: LANGSCAPE integrates partners all over the world and thus actively supports distinct as well as comparative approaches to research.

- Accessibility and Transfer: LANGSCAPE makes its international expertise accessible by sharing its results with local knowledge in research, education, and training.

- Building Excellence: LANGSCAPE strengthens research consortia at national and international levels.

Thus, LANGSCAPE counteracts tendencies of linguistic, cultural, and methodological fragmentation in foreign language research by strengthening plurilingualism, diversity and multiliteracies, and by giving special consideration to the social impact of mobility, migration, media and gender as expressions of linguistic and cultural empowerment and integration. The research concept projects beyond disciplinary and geographic boundaries. In spite of the widely acknowledged fact that language skills are one of the key resources of identity formation, the nexus between language skills and the formation of social identity and plurilingualism is a key issue for LANGSCAPE researchers. 
Consequently, the current main research focus is on Identity construction in language education. Within this research focus, LANGSCAPE distinguishes the following three pillars:

- Contexts of mobility and ecology of multilingualism

- Language learning/teaching in formal settings

- Teacher education and professionalization

Several expert meetings have been organized over the past few years to exchange research results on these topics and to bring together researchers interested in related fields of investigation. The articles published in this volume reflect this intensive and still on-going research process which has, in the meantime, led to the organisation of smaller international research teams, among these the Comenius-funded MuViT-project (Germany, Spain, Turkey), the French-German INFRAL-project funded by DAAD/PROCOPE, the blended learning project for teacher-students partnered by Paris and Siegen, which in the meantime has expanded to Berlin, and the overarching project Existing in languages with partners from China, France, Germany and Turkey.

In addition, diverse other projects have been developed at the local level by individuals or teams of researchers. They are also represented in this volume. They fittingly complement the research on plurilingualism, multiliteracies and identity construction in language learning and teaching as fostered by LANGSCAPE in general.

\section{LANGSCAPE - Réseau de recherche international sur l'acquisition et l'apprentissage des langues}

\section{Dagmar Abendroth-Timmer / Gerhard Bach / Eva-Maria Hennig}

LANGSCAPE a commencé les recherches sur l'acquisition et sur l'apprentissage des langues avec un groupe principal de partenaires européens en 1998 qui depuis a été continuellement élargi. LANGSCAPE est un réseau international de chercheurs qui se qualifie d'une propre structure ouverte. Les ambitions en recherche de Gisèle Holtzer, Gerhard Bach et de Michael Wendt ont amené à la réalisation du projet, qui a été marqué et développé par de nombreux collègues au niveau régional et international.

En 2009, Dagmar Abendroth-Timmer a pris la fonction de coordinatrice, dans laquelle elle était soutenue par l'ancien coordinateur Gerhard Bach en tant que conseiller de recherches scientifiques. Le coordinateur de LANGSCAPE est responsable de l'accessibilité des informations entre les partenaires et les 
intéressés, et de l'interconnexion des membres. Egalement il suggère de nouveaux projets de recherche et soutient les congrès par la contribution de l'office de LANGSCAPE qui assure la transmission d'informations à travers le réseau de chercheurs et l'organisation des congrès. L'office était dirigé de 2006 à 2009 par Lena Bellingrodt (Bremen) et de 2009 à 2013 par Eva-Maria Hennig (Siegen), ce qui a amené à l'institution de deux postes de doctorantes financés par les universités de Bremen et de Siegen. La grande qualité de toutes les activités de recherche entreprises par LANGSCAPE est garantie en premier lieu par le conseiller de recherches. Un nombre représentatif de partenaires apporte leur expertise aux activités de LANGSCAPE au comité directeur international. Depuis 2013, LANGSCAPE est poursuivi sous la direction de Stephan Breidbach et Lutz Küster à Berlin.

LANGSCAPE a organisé depuis 1999 quatorze congrès internationaux dont les conférences ont été publiées par des groupes d'éditeurs internationaux sous forme de recueils plurilingues dans la série Kolloquium Fremdsprachenunterricht (KFU) de la maison d'édition de Peter Lang. Beaucoup d'autres congrès ont été organisés par des membres de LANGSCAPE dans leurs régions. En 2009, des rencontres d'experts ont été établies afin de former des groupes principaux pour décider de la future orientation de LANGSCAPE. Par le biais des mobilités offertes par Erasmus on a pu découvrir différents contextes de recherche, d'autres systèmes d'éducation ainsi que d'autres universités. Les mobilités Erasmus ont également favorisé les co-tutelles dans le cadre binational ce qui a rendu possible l'échange du savoir des experts et la transmission productive de résultats et d'exemples de la pratique qui servent de modèles (samples of good practice). Grâce à ses activités, LANGSCAPE offre aux futurs chercheurs la possibilité de découvrir d'autres approches, de remettre en question leurs méthodes de recherche et d'être associés sur le plan international.

Lors de cette collaboration, LANGSCAPE poursuit les principes suivants:

- Plurilinguisme: LANGSCAPE se considère comme une communauté internationale de chercheurs. A la base de cette compétence se développent des stratégies pour agir contre une ségrégation au niveau des langues. En égard de ce même objectif, les principes de base du plurilinguisme destinés à la recherche coopérative ainsi qu'aux programmes de promotion transnationaux ont été fixés.

- Diversité et interconnexion: LANGSCAPE regroupe des partenaires du monde entier afin de comparer et d'appliquer les différentes approches à la recherche.

- Accès et transmission: LANGSCAPE rend accessible au niveau local un savoir technique international sur la recherche, l'éducation et la formation continue.

- Compétence: LANGSCAPE renforce le regroupement des membres pour des projets de recherche au niveau national et international. 
Les femmes et hommes chercheurs veulent agir contre une ségrégation linguistique, culturelle et méthodique dans la recherche sur les langues étrangères en renforçant le plurilinguisme, la diversité et la multilittératie: Poursuivant les principes de l'intégration et de la compétence linguistique et culturelle, ils accordent une attention particulière aux enjeux de la mobilité, de la migration, des médias et du gender, ce qui est fondé sur la notion d'une recherche qui transcende les frontières géographiques et disciplinaires. S'appuyant sur l'hypothèse que les compétences linguistiques sont la base centrale de la formation d'une identité, les femmes et hommes chercheurs de LANGSCAPE analysent profondément le rapport entre les compétences linguistiques et la formation d'une identité sociale et d'un plurilinguisme.

Pour cela, le groupe de chercheurs se focalise en premier lieu actuellement sur le recensement de procédés de la formation d'identités dans l'éducation linguistique. Dans ce domaine de recherche, LANGSCAPE distingue trois piliers:

- Des contextes de mobilité et de la politique des langues

- L'enseignement et l'apprentissage institutionnels de langues

- La formation et la professionnalité des enseignants de langues

Dans le but d'échanger les résultats des séries de thèmes et pour rassembler les chercheurs travaillant dans des domaines similaires, une série de regroupements d'experts a été organisée. Les contributions du recueil présent reflètent le résultat de ce processus de recherches intensif et perpétuel, qui a amené au regroupement de petites équipes internationales de chercheurs. Il faut mentionner dans ce contexte le projet-Comenius MuVit (l'Allemagne, l'Espagne, la Turquie), ainsi que le projet franco-allemand INFRAL qui a été financé par DAAD/ PRO-COPE, de même que le projet en tandems binationaux entre les étudiants de Paris et Siegen, et entre-temps aussi Berlin, ainsi que pour conclure le plus grand projet Existing in Languages géré par des partenaires en Chine, France, Allemagne et Turquie.

Vous trouverez également dans ce recueil une multitude de projets locaux et de projets indépendants qui complètent la recherche sur le plurilinguisme, la multilittératie et la formation d'identités dans l'enseignement et l'apprentissage des langues. 


\section{LANGSCAPE - Grupo internacional de investigación sobre la adquisición y la enseñanza de lenguas}

\section{Dagmar Abendroth-Timmer / Gerhard Bach / Eva-Maria Hennig}

LANGSCAPE empezó en 1998 su actividad en el campo de la investigación sobre la adquisición y el aprendizaje de lenguas con un núcleo de asociados europeos y ha ido creciendo de forma continuada. LANGSCAPE es un grupo de investigación internacional con una estructura abierta y en forma de red. Surgió de los deseos investigadores de Gisèle Holtzer, Gerhard Bach y Michael Wendt y muchos compañeros y compañeras de sus instituciones respectivas y otros a una escala internacional ayudaron a crearlo y a darle cuerpo. En 2009 Dagmar Abendroth-Timmer asumió la función de coordinadora, apoyada por el anterior coordinador Gerhard Bach como asesor científico. El coordinador de LANGSCAPE tiene la responsabilidad de hacer la información accesible para los asociados y las personas interesadas, de conectar a sus miembros, de animar los proyectos de investigación actuales y de estimular las jornadas de investigación. La oficina de LANGSCAPE apoya todo este trabajo, que garantiza la distribución de la información entre el conjunto de investigadores y garantiza la organización de jornadas. Estas tareas fueron llevadas a cabo de 2006 a 2009 por Lena Bellingrodt en Bremen y de 2009 a 2013 por Eva-Maria Hennig en Siegen. Para ello se crearon dos plazas de doctorado financiadas por las universidades de Bremen y Siegen. Un asesor de investigación apoya la gran calidad de todas las actividades investigadoras de LANGSCAPE y un número representativo de socios de la directiva internacional aportan su experiencia a las actividades de LANGSCAPE. Desde 2013 Stephan Breidbach y Lutz Küster dirigen LANGSCAPE desde Berlín.

Desde 1999 LANGSCAPE ha celebrado 14 jornadas de investigación internacionales que han sido publicadas por grupos editoriales en antologías en varios idiomas en la colección Kolloquium Fremdsprachenunterricht (KFU) (Coloquio sobre el aula de lenguas extranjeras) en la editorial Peter Lang. Algunos socios de LANGSCAPE también organizaron otros muchos congresos y en 2009 se introdujeron los encuentros de expertos para formar grupos que decidieran acerca de la reorganización de LANGSCAPE. Con el objetivo de conocer otros contextos de investigación, nuevos sistemas educativos y otras universidades, además de asesorar a doctorandos y doctorandas en programas de doctorado binacionales (co-tutelle) se utilizaron los programas de movilidad Erasmus. De esta forma, LANGSCAPE posibilita el intercambio de conocimientos entre expertos y una transmisión productiva de resultados de investigación y de ejemplos de prácticas investigadoras que se toman como modelo (samples of good practice). Las actividades de LANGSCAPE ofrecen a los jóvenes investigadores e investigadoras 
la posibilidad de conocer otros enfoques de investigación, de preguntarse acerca de sus métodos de investigación y de integrarse a un nivel internacional. Con este trabajo conjunto el grupo de investigación de LANGSCAPE persigue los siguientes principios:

- Plurilingüismo: LANGSCAPE se considera una agrupación plurilingüe. Con esta competencia como base se desarrollan estrategias que contrarresten las fronteras lingüísticas. Se han establecido principios básicos de plurilingüismo para la investigación internacional y los programas de doctorado transnacionales.

- Diversidad e interconexión: LANGSCAPE une a socios de todo el mundo con el objetivo de comparar e introducir distintos métodos de investigación.

- Acceso y transferencia: LANGSCAPE acerca los conocimientos técnicos internacionales a un nivel local para la investigación, la formación y la formación continua.

- Competencia: LANGSCAPE fomenta la unión entre los miembros de los proyectos de investigación a una escala nacional e internacional.

Los investigadores y las investigadoras quieren contrarrestar las barreras lingüísticas, culturales y metódicas de la investigación en lenguas extranjeras fomentando el plurilingüismo, la diversidad y la multiliteralidad. Para conseguir capacitación lingüística y cultural e integración le dan mucha importancia a la movilidad, la migración, los medios de comunicación y la igualdad entre géneros. Para ellos su idea de la investigación traspasa las fronteras geográficas y de cada especialidad. Con la suposición como base de que las capacidades lingüísticas representan el fundamento de la formación de la propia identidad, los investigadores y las investigadoras de LANGSCAPE se ocupan en detalle de la relación entre las capacidades lingüísticas y la construcción de la identidad social y el plurilingüismo.

Por ello el tema central del grupo de investigación es el registro de los procesos de la construcción de la identidad en la adquisición y enseñanza de lenguas. Dentro de este ámbito de investigación LANGSCAPE diferencia tres grandes pilares:

- El contexto de movilidad y las políticas lingüísticas

- El aprendizaje y la enseñanza institucionales de las lenguas

- La formación de profesores y su profesionalidad

Para intercambiar resultados acerca de estos temas y acercar a investigadores e investigadoras que están investigando en ámbitos parecidos se organizaron una serie de encuentros de expertos. Los artículos de este volumen ilustran el resultado de este intensivo y perdurable proceso de investigación que dio lugar a la fusión de pequeños equipos de investigación internacionales. Entre ellos hay 
que mencionar el proyecto MuVit, (Alemania, España, Turquía) promovido por el programa Comenius, o el proyecto franco-alemán INFRAL, financiado por DAAD/PROCOPE, el proyecto de blended learning con estudiantes de magisterio de París y Siegen y temporalmente también de Berlín y el mayor proyecto, Existing in Languages, con socios de China, Francia, Alemania y Turquía.

En este volumen se presentan un sinnúmero de proyectos locales e individuales que completan la investigación sobre plurilingüismo, multiliteralidad y construcción de la identidad en el aprendizaje y la enseñanza de lenguas.

\section{LANGSCAPE - Dil eğitimi konusunda uluslar arası araştırmacı grubu}

\section{Dagmar Abendroth-Timmer / Gerhard Bach / Eva-Maria Hennig}

Avrupa'nın birçok farklı ülkesinden araştırmacıların bir araya gelmesi ile 1998 yılında dil edinimi ve dil öğretimi ile ilgili bilimsel araştırma faaliyetlerine başlayan LANGSCAPE grubu günümüze kadar etki ve faaliyet alanını genişletmiştir. LANGSCAPE grubu ağ örgüsü (network) özellikleri taşımaktadır. Gisele Holtzer, Gerhard Bach ve Michael Wendt'in araştırmacı ruhlarına dayanarak ve onların ön ayak olması ile ilk çalışmalarına başlayan LANGSCAPE grubu hızla büyüyerek ulusal ve uluslararası bir çok araştırmacının desteği ile gelişmiş ve büyümüştür. Gerhard Bach'ın bilimsel danışmanlığı altında 2009 yılında Dagmar Abendroth-Timmer, çalışma grubunun/ağının koordinasyonunu üstlenmiştir. Langscape koordinatörü birçok görevden sorumludur, bunların arasında partner ve konu ile ilgilenen kişilere bilgi aktarmak, üyeleri biraraya getirmek, ortak araştırma projeleri ve ortak toplantılar yapmayı teşvik etmek yer almaktadır. Tüm bu çalışmalar LANGSCAPE ofisi tarafından desteklenmek ve yürütülmektedir. LANGSCAPE ofis çalışmaları 2006 ile 2009 yılları arasında Lena Bellingrodt (Bremen) ve 2009 ile 2013 yılları arasinda ise Eva-Maria Hennig (Siegen) tarafindan yürütüldü. Böylelikle Bremen ve Siegen Üniversiteleri tarafindan finanse edilen asistanlık görevleri oluşturuldu.

LANGSCAPE grubu/ağ1 üyeleri tarafından yürütülmekte olan tüm nitelikli araştırma ve çalışmalar bilimsel danışmanlar tarafindan desteklenmektedir. Ülke partnerlerinin temsilcilerinden oluşan LANGSCAPE'in uluslararası yönetim kurulunda her bir üye kendi uzmanlığını çalışmalara katarak farklı uzmanlık alanlarının bir araya gelmesini sağlanmaktadır. Nisan 2013 yılından beri LANGSCAPE faaliyetleri Berlin'den Stephan Breidbach ve Lutz Küster tarafından devam ettirilmektedir. 
1999 y1lından başlayarak LANGSCAPE 14 farklı bilimsel toplantı düzenlemiştir. $\mathrm{Bu}$ toplantılarda sunulan bildirilerin birçoğu Kolloquium Fremdsprachenunterricht (KFU) adı altında bir seri yayın halinde Peter Lang Verlag tarafından yayınlanmışırı.

Bunun yanısira sayısız kongre ve toplantılar da LANGSCAPE'in üyeleri tarafindan düzenlenmiş ve gerçekleştirilmiştir. 2009 yılından başlayarak düzenlenen uzman toplantılarında ise alt çalışma grupları oluşturularak LANGSCAPE' in yapılandırılmasına ilişkin fikirler yürütülerek uygulanmıştır.

Farklı araştırma ortamları, farklı eğitim-öğretim modelleri ve üniversiteleri tanıma ve görme ile çift danışmanlı uluslararası doktora çalışmaları (co-tutelle) başlatabilmek için Erasmus programı ile LANGSCAPE uzman değişimini, araştırma çalışmalarını ve bu çalışmaların sonuçlarının ve model oluşturabilecek iyi uygulama örneklerinin paylaşılmasını sağlanmaktadır.

LANGSCAPE faaliyetleri genç araştırmacılara farklı araştırma yaklaşımlarını tanıma ve araştırma yöntemlerini sorgulama olanağı vererek genç araştırmacıların uluslararası bilim dünyasını tanımaya ve onunla bütünleşmesine olanak sağlamaktadır.

Ortaklaşa yürütülen tüm bu faaliyet ve etkinliklerde LANGSCAPE aşağıdaki ilkeleri gözetmektedir.

- Çok dillilik: LANGSCAPE kendini çok dilli araştırma grubu olarak tanımlamaktadır. Bu ilke doğrultusunda dilsel engelleri aşmak için stratejiler geliştirmek ve ortak araştırma için uluslararası doktora programları için çok dilliliğin temel ilkelerini oluşturmaktır.

- Çeşitlilik ve bağlantılar kurmak: LANGSCAPE farklı bilimsel yaklaşımları karşılaştırmak ve uygulamak amacı ile farklı ülkelerden araştırmacıyı bir araya getirmektedir.

- Erişebilirlik ve aktarım: LANGSCAPE uluslararası düzlemdeki bilimsel bilgiyi araştırma ve eğitim için erişebilir yapmaktadır.

- Yeti: LANGSCAPE ulusal ve uluslararası platformlardaki bilimsel projeler için üyelerinin birlikteliğini güçlendirmektedir.

LANGSCAPE üyeleri çok dilliliği, dil çeşitliliğini ve çoklu okuryazarlığı güçlendirererek dilsel, kültürel ve yöntemsel sınırlamalara karşı çıkmak istemektedirler. Temelinde alan ve coğrafi sınırları aşmayı hedefleyen bir yaklaşımı izleyen LANGSCAPE, dilsel ve kültürel boyutta eylemde bulunmak ve bütünleşmek için hareketliliği, göç olgusunu, medya ve cinsiyeti özellikle vurgulamak istemektedirler.

Dilsel yetilerin kişilik oluşumunun temelini oluşturmaktadır düşüncesinden hareketle LANGSCAPE'in araştırmacıları dilsel yetiler ile sosyal kimliğin ve çokdilliliğin oluşturulması arasındaki bağlantıyı irdelemketedirler. 
$\mathrm{Bu}$ nedenden dolayı araştırmacı grubun şu anki çalışma alanı Dil ögretiminde kimlik oluşumu sürecinin anlaşılması ve araştırmasıdır. Bu araştırma alanı kendi içinde üç önemli konuyu irdelemektedir.

- Hareketlilik ve dil politikaları

- Dil öğrenimi ve öğretimi

- Öğretmen yetiştirimi ve uzmanlık

$\mathrm{Bu}$ konuları tartışmaya açmak ve farklı boytlarını diğer araştırmacılar ile irdelemek amacı ile bir dizi uzman toplantısı düzenlenmiştir. Bu kitapta yer alan makalelerin tümü bu verimli ve sürekli araştırma süreçlerinin ürünü olarak ortaya çıkmış bulunmaktadır. Tüm bu çalışmalar bir dizi uluslararası araştırma gruplarının oluşmasına neden olmuştur.

$\mathrm{Bu}$ çalışmalardan bazıları şu şekilde özetlenebilir. Comenius Projesi olarak desteklenmekte olan MuVit-Projesi (Almanya, İspanya, Türkiye), DAAD/PROCOPE tarafindan finanse edilen Fransız-Alman ortak projesi olan INFRALProjekt. Paris ve Siegen'den, zaman zaman da Berlin'den öğretmen adaylarının katılımı ile gerçekleştirilen blended-learning projesi. Ayrıca Çin, Fransa, Almanya ve Türkiye'den katılımcıların yer aldığı Existing in languages projesi.

Tüm bu konuların yanı sıra dil öğretimi ve öğreniminde çokdillilik, çoklu okuryazarlık ve kimlik oluşumnunu konu alan bir dizi ulusal/bölgesel ve bireysel projelerle ilgili yazılar da bu kitapta yer almaktadır. 


\section{Introduction}





\title{
Plurilingualism and Multiliteracies: Identity Construction in Language Education
}

\author{
Dagmar Abendroth-Timmer / Eva-Maria Hennig (Siegen)
}

Touché par le phénomène de la globalisation, l'enseignement des langues doit faire face à un défi particulier. Le développement des technologies de communication, la mobilité transnationale croissante et l'ampleur de l'anglais en tant que lingua franca (tout comme d'autres langues enseignées comme p. ex. l'espagnol) agit énormément sur le processus de formation d'identité des enseignants et des apprenants, et au-delà sur le procédé d'apprentissage. Finalement, ces développements influencent aussi les exigences de formation des enseignants. L'article suivant offre un aperçu théorique sur tous ces aspects qui dans les articles suivants seront approfondis, remis en question et complétés de façon théorique et empirique.

Fremdsprachenbildung steht in Zeiten der Globalisierung vor besonderen Herausforderungen. Die weltweite Entwicklung von Kommunikationstechnologien, die steigende transnationale Mobilität und die zunehmende Bedeutung von Englisch als Lingua franca (oder auch weiterer Schulfremdsprachen wie z. B. Spanisch) beeinflussen maßgeblich Prozesse der Identitätskonstruktion von Fremdsprachenlernenden und Fremdsprachenlehrenden sowie darüber hinaus unterrichtliche Lehr- und Lernprozesse. Schließlich wirken sich diese Veränderungen auch auf Erfordernisse der Lehrerbildung aus. Der folgende Artikel liefert einen Überblick über diese Aspekte, die in den weiteren Artikeln theoretisch und empirisch vertieft, hinterfragt und ergänzt werden.

\section{$1 \quad$ Plurilingualism ${ }^{1}$ and multiculturalism in European educa- tional systems and beyond}

In times of globalization and of cultural and linguistic hybridization, communication processes change. At all institutional levels, learners and teachers come into contact with numerous different languages, language varieties, cultures, communication tools and text genres (cf. Pauwels in this volume). They have various social backgrounds and are exposed to diverse or even contradicting views of the world which highly impact their learning processes (cf. Vygotskij 1978) or notions of 'good' teaching. In everyday life, learners as well as teachers have to cope with heterogeneous communicative situations and cannot always rely on competences or skills they have acquired through earlier experiences (cf. The New London Group 1996). Consequently,

\footnotetext{
${ }^{1}$ The term 'multilingualism' refers to the coexistence of several languages within one society, whereas the term 'plurilingualism' rather refers to the individual and his or her identity concept (cf. Cuq 2003).
} 
both, teachers and learners can be defined as "plural actors", as the French sociologist Lahire explains (2011: XI):

The scientific programme I offer here in The Plural Actor is that of a sociology that is indissociably both dispositional and contextual. It consists in taking into account the embodied past, the earlier socialising experience of the actors studied (experiences that are crystallized in the form of more or less strong and constant dispositions dispositions to believe, think and act in a certain fashion), while not claiming that we are at every moment $[\ldots]$, in every one of our acts - in a kind of synthesis of everything that we have previously experienced $[\ldots]$.

Against the background of these assumptions, we define individuals as social practitioners who are able to reflect meaning and to adapt their behavior to contextual or situational demands (cf. Lahire 2001: 132). Individuals internalize meaning on the basis of their individual dispositions, i. e. attitudes, emotions, motivations, interests, self-confidence and estimations concerning one's own self-efficacy etc. (cf. Berger/Luckmann 2007; Krüger 2011; Lahire 2011). They reflect and judge social meaning and position themselves (Harré/van Langenhove 1999; Lahire 2001).

In a globalized multilingual world, not all learners have equal access to linguistic, economic and cultural resources (cf. Bourdieu 1979). Consequently, not all of them have the chance to be heard and to express their attitudes, learning needs or feelings. Educational institutions favor and support certain languages, whereas other languages are excluded from everyday practice (cf. Abendroth-Timmer/Fäcke 2011). This is often due to the prestige which is attributed to languages (cf. Gogolin 2007) because of economic and political reasons. Learners often know (consciously or unconsciously) which languages they are supposed to speak and which ones they should rather avoid in educational contexts (cf. Gogolin 2007: 63). This is why, according to $\mathrm{Hu}$ (2003), multilingual speakers are sometimes less self-confident and therefore in turn less successful in language learning. Language education should counteract these tendencies by promoting linguistic and cultural identity formation processes in a favorable way and by supporting the esteem of diversity and alterity (cf. Council of Europe 2001). The aim is to support the personal development of all learners by integrating their linguistic and cultural resources (languages of origin, foreign languages, regional and minority languages, dialects). Linguistic and cultural diversity should be regarded as a source of enrichment which needs to be protected against tendencies of discrimination and prejudice (cf. Council of Europe 2001).

Different nations and societies have reacted differently to the societal developments mentioned so far at political and institutional levels. Education policy determines, e. g., which languages are to be taught, the time quotas available for teaching, the competences which should be acquired and how learning outcomes are to be achieved. In the European context, The Common 
European Framework of Reference for Languages (Council of Europe 2001) has been of considerable importance. The Council of Europe (2001) demands of all its members to protect languages of origin, regional languages and dialects. All Europeans are encouraged to develop plurilingual identities (mother tongues plus two foreign languages). Minority languages and languages of migrants, e. g., should not be artificially suppressed in educational contexts, but they should be connected to the target language conveyed in the classroom in a more favorable way. It is obvious that these aims are far from being achieved (cf. Fäcke 2008; cf. Fäcke in this volume) and that more research is needed in this field.

Some nations are multilingual not only because of migration processes but also on a political level, and they therefore foster several official languages. Within these countries, as e. g. in Luxembourg (cf. Ehrhart in this volume) or in Belgium, language learners continuously act as plurilingual social practitioners in multilingual institutional contexts. Forms and organizational structures of educational institutions are closely linked to linguistic policy. Researchers should explore the ways in which linguistic diversity is conceptualized and treated in multilingual school or university systems from a pedagogical and political perspective, as well as the way linguistic diversity is experienced by children, their teachers and parents. Institutional structures, procedures and tools should be adapted to the professionalization of multilingualism in order to ensure full participation within multilingual societies. The didactics of multilingualism (cf. Bär 2009) as well as the concept of multiliteracies (cf. The New London Group 1996) are of special interest within this context.

Multilingual communication processes also affect research on foreign language education itself. English has emerged as a lingua franca (cf. Gnutzmann et al. in this volume). As a consequence, writing and publishing in English has become the norm in almost all scientific disciplines. Since journal publications in English have become the essential indicator for research performance and assessment internationally, it seems obvious that non-native English researchers have to cope with an additional problem - a fact which very often puts them at a communicative disadvantage. Consequently, research in this field of interest should first explore the difficulties researchers face in this context in order to develop strategies to cope with this new demand.

Before detailing the demands all of this has on foreign language education, some definitions and models of identity need to be described. They will be evaluated with regard to their usefulness in researching and modeling linguistic, cultural and professional processes of education - i. e. identity construction processes - in heterogeneous contexts. 


\section{Models of identity}

There are numerous definitions and models of identity. Therefore, it is not possible to summarize the entire scientific discourse in this introduction. Instead, we are going to present some approaches which are of special interest with regard to the fields of research explored by LANGSCAPE members. (Empirical) studies dealing with the topic of identity always have to define what is actually implied by this term. Norton/Toohey (2011: 413) state an explosion of research activities dealing with the topic of identity in the last fifteen years. This seems to be connected to discourses on multilingual learning and on the construction of plurilingual (cf. Hu 2003) or transcultural identities (cf. Schumann 2008).

The discourse on identity mainly emerged in the period of modernism (cf. Klika 2000: 294) when people began traveling and exploring other countries. In the period of enlightenment, people in German-speaking parts of Europe also began to define the notion of 'culture' (cf. Altmayer 1997: 2). In a parallel development, the term 'civilization' was used (cf. ibid.) in French, English or other European languages. Science, education and the detachment from clerical and aristocratic authorities not only changed people's lives but also their ways of thinking. Philosophers tried to define the notion of 'subjectivity' which had not been present before. According to Descartes the 'I' is the subject of cognition, for Fichte it is the subject of action who never reaches the collective Geist, Kant connects identity to the ideal of morality, Marx relates the 'I' to a person's behavior in the social world which is structured according to social conditions (cf. Kon 1983 according to Krewer/Eckensberger 1998: 574). These and various other philosophical discourses on identity have been picked up by sociological and educational disciplines. As a result, three different movements emerged in these contexts: a biological, a psychological and a socio-dialogical concept of identity (cf. ibid.).

From a psychological perspective, adolescence is the sensitive period for the development of the 'I'. In this period, developments in the frontal part of the brain, which regulates emotions, cognitions and socially adequate behavior, enable the individual to be more self-reflective (cf. Oerter/Dreher 2008: 304). In sociological approaches, self-reflection is also crucial for defining identity. Mead (2005) differentiates the psychological 'I' from the social 'me'. The 'I' contains the subconscious needs of the individual, whereas the social 'me' reflects the impact of society on the individual. Consequently, identity emerges from a dialogical process of communication and reflection. The 'me' limits the personal freedom of development. While playing 'father, mother, child', for instance, children take over the perspectives of different social roles. In this process, they test various 'mes' and thus learn to preview how another person might act and think (cf. Mead 2005: 192). This enables them to adapt their behavior to social norms. Erikson (2011) proposes a similar dialogical model of 
identity. He also differentiates between 'I' and 'me', but in contrast to Mead's theory, his ideas are closely linked to Freud's psychosexual theory. According to Erikson (2011), the 'I' is the feeling of a coherent unity as regards the multiple experiences of the 'I'. The problem connected with this theory is that it is linked to ideas which arose in post-war times and are not up to date in our postmodernist era.

In contrast to Erikson's modernist theory of identity, poststructuralist and postmodernist theories rather highlight the disconnectedness of several parts of a person's identity. In times of multiculturalism and plurilingualism, the notion of identity is becoming fuzzier. Compound structures such as "patchwork identities" or "fluid identities" try to illustrate how identity is structured (cf. Elkind 1990): it is regarded as a set of fragments which is in a continuous process of shift and reorganization. According to socio-constructivist theories, these identities are created through communicative processes in various social contexts (cf. Altmayer 2002; Berger/Luckmann 2007; Klika 2000: 295; Mead 2005). Migration as well as educational and professional mobility contribute to the multiplication of possible social experiences. Languages and cultures merge and this in turn leads to the development of plurilingual (cf. Kramsch 2009) and transcultural identities (cf. Welsch 1999).

In addition, linguistic, cultural and economic resources are unevenly distributed within modern societies. Therefore, the social space is not only linguistically and culturally fragmented but also in a socioeconomic way. All in all, referring to socio-constructivist theories, this is not meant to say that the individual is forced to cling to social meaning. Social meaning can only become relevant for the individual if it is internalized. The concept of 'habits' (cf. Bourdieu 1979) describes these internalization processes (cf. Krüger 2011). In a further development, Lahire (2001: 131) reflects and expands on Bourdieu's theory of habits. He is predominantly interested in the question of how social meaning is internalized. Despite of social connections, the individual develops individual dispositions (attitudes, interests, motivations, emotions etc.) in social contexts. These dispositions form the repertoire of competences and skills from which the 'I' can choose in different situations. As different situations demand contradicting dispositions, the individual can also develop contradicting attitudes. Consequently, in a postmodern society, identity should no longer be defined as a stable product but as a situational and contextual construct which is influenced by the individual's dispositions developed in social interaction.

Defining identity according to Krewer/Eckensberger (1991) as a construct consisting of the individual's self-concept, his or her self-esteem and his or her self-confidence, linguistic, cultural and social identity can be described on the basis of the following categories: 
- Self-concept: What languages does the individual speak in which contexts and in which situations? How does the individual define him- or herself as a linguistic and cultural person? How does the individual describe and define his or her (plurilingual) communication practices? In which social contexts does the individual live? What is his/her (socioeconomic/linguistic etc.) status in society?

- Self-esteem: What value and prestige does the individual assign to his or her languages and the respective (socio-)cultural contexts and in what way is this estimation influenced by migration contexts or intercultural contact experiences? How is the individual viewed by people having more/less access to power in society?

- Self-confidence: How does the individual perceive and evaluate his or her competences with regard to his or her different languages? In how far can the individual contribute to changes in society?

Languages are crucial for constructing identity because they do not only convey meaning in a literal sense but they also convey norms, attitudes, beliefs about what is normal, esthetic or ethnic beliefs and cultural memories (cf. Hu 2007: 2). Depending on what languages individuals speak, they might define themselves in completely different ways (cf. Kramsch 2009). This fact also impacts foreign language learners and teachers who not only learn or convey a language system but who gradually become members of different discourse communities (cf. Chic; Pennington/Sachdev/Lau; Weyreter/Viebrock, all in this volume).

\section{Identity construction in foreign language education}

\subsection{The perspective of the learner}

As mentioned above, it is crucial to understand the structure of language learner identities and the processes that lead to the construction of plurilingual identities. Research in this field should focus on how learning environments, methods and tasks impact the formation of plurilingual and pluricultural identity constructs in formal and informal foreign language learning contexts. There is a need for a fundamental theory in foreign language education which is sensitive to issues of language learner identities (cf. Breidbach/Küster in this volume). Nevertheless, identity discourses in educational sciences do not only describe what identity is, but also what it should be and how it could be developed. It thus has a normative character (cf. Klika 2000: 296). In the last decades, several didactic approaches have been proposed which focus on how to foster the development of different identity concepts in foreign language classrooms.

The communicative approach of intercultural learning (cf. Byram 1997) proposes the model of the "intercultural speaker" as identity concept (cf. ibid.). 
The intercultural speaker should be open and curious with regard to cultural and linguistic otherness and willing to reflect his/her own cultural perspectives (savoir être). Besides, he/she should be able to interpret different perspectives in their cultural contexts (savoir comprendre) and to gain knowledge about different cultures and interactional processes on an individual and a societal level (savoir). As language is a crucial part of cultural identity and the means to negotiate and to transmit culture, the intercultural speaker should also dispose crosslinguistic knowledge. Furthermore, he/she has to be able to detect ideological and stereotypical ways of thinking (savoir s'engager) and should be able to learn autonomously by developing efficient strategies which help to cope with intercultural communication processes (savoir apprendre/faire). He/she develops a critical cultural and political awareness which allows him or her to protect diversity and otherness him- or herself.

Bennett (1986) rather focuses on the affective-cognitive pillar of intercultural learning and describes the stages learners pass through while developing an "intercultural sensitivity", i. e. the capacity to integrate different cultural perspectives and to switch between perspectives and the respective language system in a spontaneous and flexible way. Thereby, learners are enabled to cope with a world characterized by hybridization and to live together peacefully. This identity concept is mainly referred to in hermeneutic approaches of intercultural learning similar to the Didaktik des Fremdverstehens developed by the graduate research group of the same name (cf. Bredella 2010). When working with texts in the foreign language classroom, students are encouraged to reflect on their own linguistic and cultural identities before temporarily taking over the perspectives of literary characters they are confronted with (e. g. by doing creative tasks through which they have to speak or write from the perspective of a figurative character; cf. Caspari 2000). In a dialogue between the student's own perspective and the perspective of the literary character, the learner is expected to overcome stereotypes and ethnocentric viewpoints (cf. Bredella 2010). In a meta-reflective process, the learner should negotiate these perspectives and integrate or reconstruct different viewpoints. Ideally, this learning process will lead to the development of empathy and openness towards different perspectives and to the development of transcultural identities (cf. Schumann 2008), in which different perspectives merge and mingle.

In the context of the didactics of multilingualism (cf. Reissner 2011), identity is usually defined on the basis of the concept of "linguistic awareness" (cf. Eichler/Nold 2007). This concept includes different domains of competence: affective competences (attitudes and emotions towards language), cognitive competences (views of the world, analysis of linguistic units), metacognitive competences (sociolinguistic awareness, the ability to detect ideologies) and communicative competences (performance) (cf. Gnutzmann1997: 232ff.). The EuroCom-Project which adds a practical dimension to this theoretical concept 
(cf. Klein/Stegmann 2000) focuses on cross-linguistic structures existing in language families (parallel syntactic structures, morpheme-grapheme relations etc.). Crosslinguistic learning strategies (cf. Bär 2009), for instance, can facilitate the decoding of a yet unknown language. Learners become more autonomous by making use of all their linguistic resources, including their languages of origin.

The steady growth of cultural and linguistic diversity is accompanied by an increased use of different communication channels and thus forces educators to rethink literacy learning and teaching. The pedagogy of multiliteracies (cf. The New London Group 1996) "overcomes the limitations of traditional approaches by emphasizing how negotiating the multiple linguistic and cultural differences in our society is central to the pragmatics of the working, civic, and private lives of students" (ibid.: 1). It includes the immersion in experience, the interpretation of knowledge, critical analyses of learning processes in connection to the respective context as well as the transfer of knowledge and the capacity to apply this to new or different contexts (ibid.: 21f.). Plurilingual literacy plays a crucial role in ensuring participation in social contexts. As societies change with regard to their linguistic and cultural heritage, the pedagogy of literacy has to change as well in order to meet the new demands of the individual. Multilingual talking books, which are books that are made on the computer and used on screen, may be the key to a first practical realization of this so far rather theoretical concept (cf. Elsner in this volume).

The identity concept which can be attributed to this didactic approach is that of a European identity as described by Küster (2007: 40). What is crucial for defining European identity is a consciousness for heterogeneity with regard to linguistic, ethnic and cultural entities and especially with regard to multi- and plurilingualism (cf. ibid.). This is what, in addition to common historical memories, unites all Europeans. All in all, Küster (ibid.) defines European identity as a continuum including individual and collective levels. The latter might range from local to global identities forming overlapping networks. Modern media of communication and digital social networks contribute to the worldwide interconnection of speakers (cf. ibid.: 41) and thus enhance the merging of different languages and cultures.

\subsection{The teacher perspective}

Professional identity can be described on the basis of individual attitudes, knowledge and teaching practices (cf. Miller 2009: 175). The teaching practices of both, student teachers and in-service teachers, do not necessarily correspond with their professional self-concept. When student teachers find themselves under pressure to act immediately, mental representations emerge (Blömeke 
2005). Furthermore, their practical realization of didactic principles and methodological guidelines might form a distinct contrast to their professional ideals.

The ongoing development of professional teacher identities therefore hinges on reflecting on what seems personally, institutionally, and socially doable in classrooms, how change is effected, and how knowledge, pedagogy, and identity intersect. (Miller 2009: 178)

Undoubtedly, it is essential that teachers become aware of these subjective limitations if they wish or need to adapt their teaching practices to their professional self-concept. Such an awareness can only be achieved through diverse reflection processes which relate scientific or theoretical knowledge to real teaching situations (cf. Frevel in this volume). In times of globalization and of corresponding learners' pluriculturalism, these processes need also refer to the question of how to deal with individual plurilingualism and multiculturalism in the language classroom.

In her study on the professional self-concept of foreign language teachers, Caspari (2003: 282) claims that there are not sufficient empirical research projects that try to explore the impact of subjective theories on the act of teaching. In this context, it is crucial to focus on teachers' (language) learning experiences, on the one hand, and their views on teachers' role in language pedagogy and educational functions, on the other (cf. Abendroth-Timmer 2011). Existing studies point to the relation between teachers' institutional learning experiences and their development of self-efficacy. It seems clear that it is on the basis of their experience that student teachers develop expectations regarding their self-efficacy. This fact must not be ignored, because these expectations tend to be rather immutable. It stands to reason that student teachers have developed assumptions about learning and teaching (of languages) which influence their willingness to deal with new learning theories and to adopt new teaching concepts. Therefore, it seems to be crucial that those assumptions can be made accessible through approaches of reflective teacher education.

A question that still remains unanswered is that of how exactly theoretical knowledge can be transferred into practical knowledge. It is assumed that such a transfer is a highly complex process in which theory and practice have to be interrelated (Herzig et al. 2005: 51f.). The reflection of individual premises thus needs to be established in teacher education at university level (cf. De Mesmaeker/Lochtman; Etuş/Schultze, all in this volume). Moreover, future foreign language teachers have to (re-)reflect on their professional identity and their teaching practices with the focus on multilingual and multicultural classrooms and with the objective to avoid a monolingual and monocultural habitus. In order to address the multicultural makeup of their future classrooms in an appropriate way, it is necessary that student teachers of foreign languages be 
exposed to intercultural and multilingual learning situations themselves. If reflected upon, these situations can potentially raise an awareness that supposedly influences the way teachers deal with the diversity of languages and cultures of their students in a positive way. Online seminars offer new options for participants with different languages and cultures to work together via learning platforms and to experience multilingual and intercultural communication processes (cf. Bechtel/Ciekanski in this volume). Experiences which are systematically reflected on the basis of theoretical knowledge might help to convert action-oriented knowledge into action-oriented competences.

Research needs to find an answer to the multifarious question of how reflection based on both theory and classroom reality can be initiated and guided in order to enable teachers (at every stage in their career) to overcome individual/subjective patterns of thinking and acting, and thus to tackle in a flexible manner the complexity of teaching situations in a pluralistic society (cf. Wildemann et al. in this volume).

\section{Perspectives for academic and practical contexts}

\subsection{Consequences for foreign language education research}

In the area of language policy, research will have to focus on governance (Bologna, European Common Framework of Reference) and educational standards. Research focusing on the foreign language learner should especially be directed towards the investigation of attitudes towards multiculturalism and plurilingualism as well as towards the investigation of learning processes. In this context, researchers should differentiate between cognitive, affective, metareflective, communicative and methodological domains (of competence). In language policy research and in the didactics of multilingualism (cf. Bär 2009), a lot of work has already been invested in this research area (cf. Baur/Hufeisen 2011) by studying the impact of the mother tongue on second language acquisition processes and by developing and investigating strategies of crosslinguistic language learning. Furthermore, research should focus on how certain learning tasks or settings impact the quality of these processes. Currently, there is still a lack of praxis-oriented work that would be able to illustrate how a pedagogy of multiliteracies would function in the (foreign language) classroom. Tasks have to be developed and tested which can foster the development of plurilingual identities and/or the development of a European identity. On a metareflective level, we can investigate how learners evaluate their learning processes on the background of certain settings (virtual, face-to-face, more or less formalized or institutionalized). As identity constructs define the preconditions for learning processes, researchers should always be sensitive to 
the learners' individual dispositions (attitudes, emotions, motivations and interests towards/on languages and cultures) and the learners' identities.

Moreover, the attitudes of teachers towards pluri- and multilingualism and towards multiculturalism have to be investigated. Pedagogical approaches which claim to change habits or individual dispositions will have to be put to the test. It is crucial that teachers become aware of multilingual didactic approaches and of the respective media that can stimulate and foster cultural und plurilingual learning. In this research area, the development of teaching methodologies and theories still remains a necessity for research in order to find out how bi- and multilingualism can be integrated into both language classrooms and teacher education. The question of the role of new media for triggering (inter)cultural and multilingual learning processes and the additional question of how they may affect plurilingual identities have to be addressed as well.

\subsection{Consequences for classroom practices}

International projects (cf. e. g. Comenius projects) foster institutional or online exchanges of learners and teachers. They ensure the cooperative work of subjects with diverse cultural and linguistic backgrounds and thereby initiate discussions on different living conditions, attitudes and beliefs or perspectives (cf. Abendroth-Timmer/Aguilar Río; Bechtel/Ciekanski; Chik; Narcy-Combes/ Narcy-Combes; Weyreter/Viebrock, all in this volume). Through these exchanges, learners and teachers are enabled to act in multilingual and multicultural learning communities with which they are supposed to identify in their role as learner or teacher. On the basis of these interactions, they are encouraged to describe, open and reflect on their cultural and linguistic identities. The results of the evaluation of these projects can then be taken back to the respective institutions (universities, schools, school administration etc.) and thus directly influence learning processes.

Plurilingualism and transcultural identities can also be developed through Content and Language Integrated Learning (CLIL) approaches. By focusing on the subject matter, the content is in the center of interest, whereas the language functions as a medium of communication and not as an object of analysis. Working with texts written in different languages can provide learners with a wide range of different perspectives. From the teacher perspective, national teaching traditions are questioned (cf. Breidbach 2004: 160 f.) and discussed. By contrasting different concepts and perspectives learners are supported to process information more deeply (cf. Bonnet 2004).

Content-orientation can also be established on the basis of task-based approaches (cf. Ellis 2003). Learners are supposed to manage semi-authentic communicative situations within complex contexts. The latter should include 
topics which seem to be relevant for learners on a personal level. In this way, language learning materials, cultural topics and diverse media become central means to elicit language learning processes which do not only depart from the learners' experiences but which are also co-determined by learners. With regard to tasks, it might be interesting to investigate how they can support not only linguistic but also cultural and multilingual learning processes. They might also be a suitable means in order to implement the theoretical program of The New London Group (1996) in the foreign language classroom. Tasks based on literature, films and music can be used in order to make students reflect upon their cultural and linguistic identity, as long as they address learners not only cognitively but also emotionally.

\section{$5 \quad$ Summary and outlook}

This introduction article can only provide a rough overview of theories, research approaches and possibilities of eliciting and investigating processes of identity construction in multilingual and multicultural language learning and teaching contexts. Nevertheless, it should be obvious that the notion of identity is not a new fashion term or fashion concept. Instead, it is able to bring together central concepts which are crucial in language education research in a fruitful way; foremost among these being multilingualism, multiculturality, intercultural and multimedia communication, multiliteracies, and the development of competences.

The articles presented in this volume are intended to depict and to elaborate on several of the aspects mentioned here. In chapter I, contexts of mobility and the ecology of multilingualism in European or national contexts will be examined. The second chapter focuses on identity construction processes in foreign language learning and teaching contexts. Finally, the third chapter focuses on professional identity constructs of novices and highly experienced teachers.

\section{References}

Abendroth-Timmer, Dagmar (2011). Reflexive Lehrerbildung: Konzepte und Perspektiven für den Einsatz von Unterrichtssimulation und Videographie. Zeitschrift für Fremdsprachenforschung 22/1: 3-41.

Abendroth-Timmer, Dagmar / Fäcke, Christiane (2011). Migrationsbedingte Mehrsprachigkeit. In: Meißner, Franz-Joseph / Krämer, Ulrich (eds.): Spanischunterricht gestalten. Wege zu Mehrsprachigkeit und Mehrkulturalität. Seelze-Velber: Klett-Kallmeyer. 16-48.

Altmayer, Claus (1997). Zum Kulturbegriff des Faches Deutsch als Fremdsprache. Zeitschrift für Interkulturellen Fremdsprachenunterricht (ZIF) 2/2: 1-25. Online: http://zif.spz.tudarmstadt.de/jg-02-2/beitrag/almayer3.htm (last retrieval [10.03.2012]). 
Altmayer, Claus (2002). Lernstrategien und autonomes Lernen. Teilaspekte eines 'konstruktivistischen' Fremdsprachenunterrichts? Babylonia 2: 7-13. Online: http://babylonia.ch /fileadmin/user_upload/documents/2003-3-4/Baby3-4_03altmayer.pdf (last retrieval [12.05.2012]).

Bär, Marcus (2009). Förderung von Mehrsprachigkeit und Lernkompetenz. Fallstudien zu Interkomprehensionsunterricht mit Schülern der Klassen 8 bis 10. Tübingen: Narr.

Baur, Rupprecht S. / Hufeisen, Britta (eds.) (2011). „Vieles ist sehr ähnlich“: Individuelle und gesellschaftiche Mehrsprachigkeit als bildungspolitische Aufgabe. Hohengehren: Schneider Verlag.

Bennett, Milton J. (1986). A developmental approach to training for intercultural sensitivity. International Journal of Intercultural Relations 10/2: 179-196.

Berger, Peter L. / Luckmann, Thomas (2007). Eine gesellschaftliche Konstruktion der Wirklichkeit. Eine Theorie der Wissenssoziologie. Frankfurt/M.: Fischer.

Blömeke, Sigrid (2005). Lehrerausbildung - Lehrerhandeln - Schülerleistungen. Perspektiven nationaler und internationaler empirischer Bildungsforschung. Antrittsvorlesung 10.12.2003. Berlin: Humboldt-Universität zu Berlin, Philosophische Fakultät IV, Institut für Erziehungswissenschaften. 1-18. Online: http://edoc.hu-berlin.de/humboldt-vl/139/ bloemeke-sigrid-3/PDF/bloemeke.pdf (last retrieval [15.03.2013]).

Bonnet, Andreas (2004). Chemie im bilingualen Unterricht. Kompetenzerwerb durch Interaktion. Wiesbaden: Verlag für Sozialwissenschaft.

Bourdieu, Pierre (1979). La distinction. Critique sociale du jugement. Paris: Minuit.

Bredella, Lothar (2010). Das Verstehen des Anderen. Tübingen: Narr.

Breidbach, Stephan (2004). Bildung, Kultur, Wissenschaft: Grundzüge einer reflexiven Wissenschaftsdidaktik für den bilingualen Sachfachunterricht. In: Bonnet, Andreas / Breidbach, Stephan (eds.): Didaktiken im Dialog: Konzepte des Lehrens und Wege des Lernens im bilingualen Sachfachunterricht. Frankfurt/M.: Lang. 153-164.

Byram, Michael (1997). Teaching and assessing intercultural communicative competence. Clevedon: Multilingual Matters.

Caspari, Daniela (2000). Kreative Textarbeit als Beitrag zum Fremdverstehen. Fremdsprachenunterricht 44/2: 81-86.

Caspari, Daniela (2003). Fremdsprachenlehrerinnen und Fremdsprachenlehrer. Studien zu ihrem beruflichen Selbstverständnis. Tübingen: Narr.

Council of Europe (2001). Common European Framework of Reference. Online: http://www. coe.int/t/dg4/linguistic/source/framework_en.pdf (last retrieval [15.03.2013]).

Cuq, Jean-Pierre (2003) (ed.). Dictionnaire de didactique du français. Langue étrangère et seconde. Paris: CLE International.

De Florio-Hansen, Inez/Hu, Adelheid (2007) (eds.): Plurilingualität und Identität. Zur Selbst- und Fremdwahrnehmung mehrsprachiger Menschen. Tübingen: Narr.

Eichler, Wolfgang / Nold, Günter (2007). Sprachbewusstheit. In: Klieme, Eckhard / Beck, Bärbel (eds.): Sprachliche Kompetenzen. Konzepte und Messung. DESI-Studie (DeutschEnglisch-Schülerleistungen-International). Weinheim, Basel: Beltz. 63-82. 
Elkind, David (1990). Total verwirrt: Teenager in der Krise. Bergisch Gladbach: Bastei Lübbe.

Ellis, Rod (2003). Task-based language learning and teaching. Oxford: Oxford University Press.

Erikson, Erik H. (2011). Identität und Lebenszyklus: drei Aufsätze. Frankfurt/M.: Suhrkamp.

Fäcke, Christiane (2008). Mehrsprachigkeit im Kontext europäischer Sprachenpolitik. In: Frings, Michael/Vetter, Eva (eds.): Mehrsprachigkeit als Schlüsselkompetenz: Theorie und Praxis in Lehr- und Lernkontexten. Akten zur gleichnamigen Sektion des XXX. Deutschen Romanistentages an der Universität Wien (22. Bis 27. September 2007). Stuttgart: ibidem. 11-26.

Gnutzmann, Claus (1997). Language Awareness. Geschichte, Grundlagen, Anwendungen. Praxis des neusprachlichen Unterrichts 44/3: 227-236.

Gogolin, Ingrid (2007). „Das ist doch kein gutes Deutsch!“ - Über Vorstellungen von guter Sprache und ihren Einfluss auf Mehrsprachigkeit. In: De Florio-Hansen, Inez/Hu, Adelheid (eds.). 59-71.

Harré, Rom/van Langenhove, Luk (1999). The dynamics of social episodes. In: Harré, Rom/van Langenhove, Luk (eds.): Positioning theory: Moral contexts of intentional action. Oxford: Blackwell. 1-14.

Herzig, Bardo / Grafe, Silke / Reinhold, Peter (2005). Reflexives Lernen mit digitalen Videos - zur Auseinandersetzung mit dem Theorie-Praxisverhältnis in der Lehrerausbildung. In: Welzel, Manuela / Stadler, Helga (eds.): Nimm doch mal die Kamera! Zur Nutzung von Videos in der Lehrerbildung - Beispiele und Empfehlungen aus den Naturwissenschaften. Münster: Waxmann. 45-64.

Hu, Adelheid (2007). Mehrsprachigkeit, Identitäts- und Kulturtheorie: Tendenzen der Konvergenz. In: De Florio-Hansen, Inez / Hu, Adelheid (eds.). 1-23.

$\mathrm{Hu}$, Adelheid (2003). Schulischer Fremdsprachenunterricht und migrationsbedingte Mehrsprachigkeit. Tübingen: Narr.

Klein, Horst G. / Stegmann, Tilbert D. (2000). EuroComRom - Die sieben Siebe. Romanische Sprachen sofort lesen können. Aachen: Shaker.

Klika, Dorle (2000). Identität - ein überholtes Konzept? Kritische Anmerkungen zu aktuellen Diskursen außerhalb und innerhalb der Erziehungswissenschaft. Zeitschrift für Erziehungswissenschaft 3: 285-301.

Korthagen, Fred A. J. (2002). Schulwirklichkeit und Lehrerbildung, Reflexion der Lehrertätigkeit. Hamburg: EB-Verlag.

Kramsch, Claire (2009). The multilingual subject: what foreign language learners say about their experience and why it matters. Oxford: Oxford University Press.

Krewer, Bernd / Eckensberger, Lutz H. (1998). Selbstentwicklung und kulturelle Identität. In: Hurrelmann, Klaus/Dieter, Ulrich (eds.): Handbuch der Sozialforschung. Weinheim [u. a.]: Beltz. 573-594.

Krüger, Paula (2011). Sprachlernhabitus und Bedeutungskonstruktion beim Fremdsprachenlernen. Frankfurt/M.: Lang. 
Küster, Lutz (2007). Multiliteralität und Europa-Identität. Zielsetzungen und Realisierungsperspektiven vor dem Hintergrund europäischer Sprach- und Bildungspolitik. In: Elsner, Daniela / Küster, Lutz / Viebrock, Britta (eds.): Fremdsprachenkompetenz für ein wachsendes Europa. Das Leitziel „,Multiliteralität“. Frankfurt/M.: Lang. 35-48.

Lahire, Bernard (2001). De la théorie de l'habitus à une sociologie psychologique. In: Lahire, Bernard (ed.): Le travail sociologique de Pierre Bourdieu. Dettes et critiques. Paris: La Découverte. 121-152.

Lahire, Bernard (2011). The Plural Actor. Cambridge: Polity Press.

Mead, George Herbert (2005). Geist, Identität und Gesellschaft aus Sicht des Sozialbehaviorismus. Frankfurt/M.: Suhrkamp.

Miller, Jennifer (2009). Teacher identity. In: Burns, Anne / Richards, Jack C. (eds.): Second Language Teacher Education. New York: Cambridge University Press. 172-181.

Norton, Bonny/ Toohey, Kelleen (2011). Identity, language learning, and social change. Language Teaching 44/4: 412-446. Online: http://journals.cambridge.org/action/display Abstract? fromPage $=$ online \&aid $=8361283$ (last retrieval [12.03.2013])

OECD (2005). DeSeCo-Projekt, Definition und Auswahl von Schlüsselkompetenzen. Zusammenfassung. Online: http://www.oecd.org/dataoecd/36/56/35693281.pdf (last retrieval [11.03.2013]).

Oerter, Rolf / Dreher, Eva (2008). Identität: das zentrale Thema des Jugendalters. In: Oerter, Rolf / Montada, Leo (eds.): Entwicklungspsychologie. Weinheim, Basel: Beltz. 303-315.

Reissner, Christina (2011): Europäische Interkomprehension und ihre institutionelle Verankerung. In: Reissner, Christina (ed.): Romanische Mehrsprachigkeit und Interkomprehension in Europa. Akten der gleichnamigen Sektion auf dem XXXI. Romanistentag 2009 an der Universität Bonn. Stuttgart: ibidem. 181-197.

Schön, Donald A. (1983). The Reflective Practitioner: how professionals think in action. London: Temple Smith.

Schumann, Adelheid (2008). Transkulturalität in der Romanistischen Literaturdidaktik. Kulturwissenschaftliche Grundlagen und didaktische Konzepte am Beispiel der "littérature beur". Fremdsprachen lehren und lernen 37: 81-94.

The New London Group (1996). A Pedagogy of Multilieracies: Designing Social Futures. Havard Educational Review 66/1. Online: http://www.hepg.org/her/abstract/290 (last retrieval [11.03.2013]).

Vygotskij, Lev S. (1978). Mind in society. The development of higher psychological processes. Cambridge: Harvard University Press.

Welsch, Wolfgang (1999). Transculturality - the Puzzling Form of Cultures Today. In: Featherstone, Mike/Lash, Scott (1999) (eds.): Spaces of Culture. City, Nation, World. London: Sage. 194-21. 

I.

Contexts of Mobility and Ecology of Multilingualism 



\title{
Rethinking the Learning of Languages in the Context of Globalisation and Hyperlingualism
}

\author{
Anne Pauwels (London)
}

In this paper the major challenges to the learning of languages in the context of globalisation are explored: increased diversification of learners' linguistic profiles and learning experiences, new communication technologies and their impact on communicative practices as well as the increased commodification of language. The main focus is on how aware and prepared university language teachers are to deal with these developments.

In diesem Beitrag werden die bedeutendsten Herausforderungen für den Fremdsprachenunterricht untersucht, die auf Globalisierungsprozesse zurückzuführen sind: die verschärfte Differenzierung der sprachlichen Lernerprofile, neue Kommunikationstechnologien und deren Einfluss auf den Sprachgebrauch und auf Kommunikationsweisen sowie die erhöhte Kommodifizierung von Sprache. Der Hauptfokus des Beitrages liegt jedoch in der Erforschung der Frage, wie bewusst sich Universitätsdozentinnen und -dozenten dieser Herausforderungen sind und wie sie damit im Unterricht zurechtkommen.

\section{Globalisation}

In one of the first volumes dealing with language teaching and globalisation, the editors note that "globalisation is nothing if not a fashionable term - it pervades contemporary political rhetoric and is a keyword of both academic and popular discourse on economy, technology and culture" (Block/Cameron 2002: 1). A decade later the use of the term has intensified as well as become omnipresent in societies around the world. Its centrality in exploring, examining and understanding developments and events is taken as self-evident. Linguistic evidence of this centrality is found in the fact that the term is now thoroughly embedded in dictionaries of languages around the world. Although there are of course differing views, perspectives and opinions about globalisation, definitions of the term tend to include similar elements: globalisation is seen as a process (social, cultural, political, economic) that has an impact on everyday life. It overcomes geographical constraints potentially reducing other boundaries or differences (social, cultural, linguistic, economic). The factors that facilitate globalisation include vastly enhanced communication and transportation technologies that have allowed for a massive increase in short-term and longterm transnational mobility of people, as well as economic and trade systems that require access to resources world-wide and that are increasingly servicebased. 
These factors of globalisation all have an impact on communication, specifically through the medium of language. Language is indeed a key element in the globalisation process: it shapes and is affected by the process.

\section{Globalisation and language(s)}

In the previous section I alerted to the important role that language plays in the globalisation process. Here we briefly touch upon three aspects: the centrality of language in the 'new' economy, the linguistic consequences of increased transnational mobility and of new communication technologies.

\subsection{Language and the 'new' economy}

The globalised 'new' economy is one that is heavily service-oriented, having moved away from a focus on manufacture and an exploitation of primary resources. Information (exchange), services around products and (symbolic) goods/artefacts are key to this economy. The provision of services as well as the assurance of quality are heavily reliant on appropriate and effective communicative and linguistic practices. This view foregrounds the 'skills' component of language and has led to an explosion in (self-help) manuals, guides and handbooks outlining what constitutes good and effective communication and how individuals and corporations can improve their skills to suit the new service economy. An increasing number of linguistic scholars have critically commented on this trend pointing out that communication training has now become de rigueur for almost every worker, even those whose jobs involve limited contact with clients, customers or colleagues (e. g. Cameron 1995, 2002; Duchêne/Heller 2012; Heller 2003). Another consequence of this focus on effective communication is the desire for a language that can operate at a global level, a global lingua franca that reduces the potential for confusion and miscommunication. Having access to this lingua franca provides the speaker with significant linguistic capital that will not only open up economic markets but also provide increased educational, employment and sociocultural opportunities. English has become that lingua franca, at least for the time being. In 2.4.1 we shall look at the impact this has had on the language learning scene.

\subsection{Hypermobility and language}

Globalisation is intricately linked to enhanced mobility largely facilitated through improved and expanded transportation systems. Transnational and transcontinental movements for education, business, employment and leisure (tourism) have increased in frequency and are affecting the lives of many more people than a few decades ago. Furthermore, improvements in transportation 
systems have allowed for much more frequent contact between people separated by vast distances: the cost of long-distance travel has come within reach of a larger sector of the population. For example, for migrants and refugees who $\mathrm{had} /$ were settled in areas far removed from their homeland, visits to and from the homeland are no longer a 'once-in-a-lifetime' occasion. Another facet of this extreme mobility, increasingly labelled 'hypermobility', is the creation of hyperdiverse metropolitan hubs and cities. Of course, cities around the world have long been sites of diversity bringing together people from all walks of life, from different countries, cultures, religions, ethnolinguistic and racial groups. Globalisation has intensified this diversity aspect of large cities, although it has also brought a 'homogenising' aspect to them. This is very well illustrated in the visual hallmarks of such cities. On one hand, their linguistic landscapes display a multitude of languages reflecting the ethnolinguistic diversity of inhabitants, yet there are also the visual icons of global, transnational fast food and coffee chains, shops, banking and trade, exuding sameness (e. g. Blommaert 2010; Gorter 2006). Linguistic practices in these sites similarly demonstrate the hallmarks of globalisation: besides a plethora of plurilingual modes practiced by large sections of the community, there is the widespread use of the 'unifying' or common linguistic code. With regard to the former, globalisation has led not only to augmented levels of multilingualism and language contact but also to more rapid and frequent changes in linguistic constellations. For example, the most recent UK Census (2011) revealed that Polish has displaced South Asian languages like Punjabi, Urdu, Bengali and Gujarati in London. These languages had dominated the London scene for quite a few decades. Globalisation and mobility have also stimulated the further establishment of diaspora communities. Many of these communities see language(s) as a key component of their identity and wish to pass the language(s) on to their offspring. These communities will also have needs to acquire the dominant language(s) of their new environment. Both these factors will have an impact on language learning in a global context.

\subsection{Language and the new communication technologies}

In a globalised world mobility is also enhanced through technological developments that overcome the real-space constraints of communication: the internet, sophisticated telephony and media systems have paved the way for 24/7 world-wide communication to be within reach of many people. These developments in communication technologies, especially those involving the internet, have had a dramatic impact on language and communication practices. It has given rise to new modes of communication such as various forms of videoconferencing, to new genres including email, blogs, short text messages (SMS), and 'tweets'. It is also leading to a marked shift in the preferred channels of communication, at least among younger generations. This generation is more 
likely to interact using technology-mediated means of communication, foregrounding quite dramatically 'written' or 'typed' modes of communication. We will see in 2.4.3 that these new communication technologies also affect the learning of other languages.

\subsection{Globalisation and the learning of languages}

The impact of globalisation is also strongly felt within the sphere of the learning of languages. A small but nevertheless remarkable consequence of globalisation and the factors contributing to it relates to the nomenclature of the field-foreign language learning. This term reflects the ideological stance that favours a 'one nation - one language' policy. Such a policy considers any language, other than the national or official language as foreign. In today's multicultural societies the linguistic landscapes are multilingual, which makes the term foreign language a misnomer. Yet it persists in both scholarly and popular contexts. In this paper, however, I will refer to the learning of 'foreign' languages (FLL) predominantly as 'language learning' (LL).

More substantial consequences of globalisation on LL occur at the level of policy and planning, as well as at the level of the actual learning (processes). Here I will focus briefly on one major impact on LL policies - the commodification of LL - leading to the global LL scene being dominated by a handful of languages. With regard to the impact on LL itself, I will briefly touch upon how learner profiles have changed or are changing, and how changes in modes of communication affect LL. My main focus, however, will be on the other key agent in the learning process - the teacher: I will examine to what extent language teachers are aware of these impacts and how they accommodate them in their teaching practices. The site for analysis of the latter is the university (tertiary education). The choice of this site is linked to the crucial role of universities in the lifelong learning process: they generate and advance (new) knowledge through research, and they integrate those advances in and disseminate them through their teaching. The recipients of this teaching process in turn disseminate these new knowledges through their own (teaching) practices.

\subsubsection{English as the desired linguistic capital}

The centrality of language and communication in the new economy has highlighted the commodification of language: language undoubtedly has market value. As the new economy is played out in a global market place, the language or languages that give(s) access to this global arena are perceived as highly desired linguistic capital. Currently, English clearly dominates this market place 
but languages like Chinese, Spanish, and Arabic are becoming major players and languages like French, Russian and German still play some role in transglobal or at least trans-regional communication. Nevertheless, English is regarded as the global language, at least for the time being. The status of English as global lingua franca has had major effects on the LL scene: English has become the language most widely studied as second, third or 'foreign' language. The number of such learners is now estimated to be over 1 billion (Graddol 2006). English occupies the status of first 'foreign' language in many countries around the world. This has led to a significant expansion of the learning of English and thus in LL per se. In some communities in which LL was not an established part of the curriculum, the decision to introduce English as a second or foreign language has indeed led to massive increases in learners. Prime examples are Japan and China, with the latter rolling out a policy of universal provision of English across the entire schooling system (Hu 2005). In communities that have an established tradition in LL (e. g. many parts of Europe), the desire to learn English has led to shifts in the hierarchy of languages on offer: often English moves up the priority ladder displacing former dominant foreign or second languages. This is for example the case in some Nordic countries where English has clearly displaced German as well as French. The 2005 Eurobarometer language survey mentioned that in 19 out of 29 countries English is the most widely spoken (learnt) language besides the mother tongue: this is especially the case in Sweden (89\%), Malta (88\%) and the Netherlands (87\%). The 2012 survey confirmed this, with only minor changes in the top countries: Netherlands $(90 \%)$, Malta $(89 \%)$ and Sweden $(86 \%)$. For Europe (EU countries), the ascendancy of English may also have affected the number of people who can communicate in two other languages: in 2005 this was $28 \%$ and by 2012 it had dropped to $25 \%$. It seems that the dominance of English and its status as global lingua franca may reduce the need to acquire skills in another language.

In communities where English is the dominant and/or official language, the status of English as the main global language is a powerful factor in the continuing struggles to increase LL. Despite strong rhetoric backed up by evidence that 'English is not enough' (e. g. Graddol 1997, 2006), Anglophone societies tend to trail in LL rankings. For example, both the United Kingdom and Ireland find themselves at the lower end of LL in Eurobarometer surveys: in 2012 Ireland was ranked last with $66 \%$ not able to speak an additional language and the United Kingdom second last with $62 \%$. Whilst these countries may have seen an expansion in the languages offered for study including some 'heritage' languages, this has not yet led to a sizeable increase in language learners.

Furthermore, the commodification of language and the view that the acquisition of another language is primarily directed at increasing one's linguistic capital continue to affect both attitudes and policies towards the 
learning of languages in education. Languages that do not rate high in terms of market value, among them many languages of ethnolinguistic minorities, immigrants and marginalised groups, may well be 'valued' in state discourses but their learning is not seen as a state responsibility and is thus often relegated to the language group or community in question. This stance has influenced the attitudes of many heritage speakers themselves internalising views that their languages do not have a place in state-based LL.

Finally, the dominant status of English in the LL scene has been supported by a massive English language learning industry that constantly produces materials, designs new curricula and modes of delivery for their products. It is also backed up by a wealth of scholarly research that explores socio-political as well as linguistic and pedagogical aspects of English language learning. Developments in the learning of other languages often draw upon findings from research on English language learning and teaching, thus strengthening further the influential role of English in LL theory and practice.

\subsubsection{New profiles of language learners at university}

The effects of globalisation and increased trans-global mobility are also strongly felt in the educational sector, especially at tertiary/university level. Within Europe, schemes such as ERASMUS actively promote student and staff mobility and expose students to different linguistic and cultural contexts. Beyond Europe, student mobility is also extensive but possibly lacks the exchange element that characterises intra-European mobility. The direction of mobility is from eastern and southern world regions to western and northern ones, especially if the latter offer education through the medium of English. The majority of universities in these regions have significant international student populations transforming their campuses into multilingual and multicultural hubs. Another contributing factor to the multilingual and multicultural nature of student populations is the increasing participation of immigrants and ethnolinguistic minorities in higher education (Pauwels 2007). It is therefore not surprising that the linguistic profile of university language learners, and university students per se, is much more diverse than it was even a few decades ago. Today's language students are likely to come from very diverse linguistic backgrounds, have varying degrees of linguistic competencies both in the target language as well as in other languages. This will also be the case for their preuniversity language learning experiences and exposures, motivation and attitudes towards language learning as well as reasons for language study.

Whilst factors such as motivation, attitudes and reasons for university language study have been examined extensively in applied and second language acquisition research (e. g. Dörnyei 2001, 2003; Gardner 1985; Gardner/Lambert 
1972), the impact of heightened diversity in the linguistic profiles and language experiences of current learners has not yet received the same attention, although more recent work on plurilingual learners by scholars such as Fenoulhet/Ros i Solé (2012), Kramsch (2006) and Zarate (2010) is starting to make a change. Particularly under-explored remains the question of how these changed learner profiles affect language teaching practices.

\subsubsection{Communication technologies and LL}

The impact of rapid advances in communication technologies on language practices and modes of communication is a major topic of research within many branches of linguistics. Among the more prominent areas are machine translation, the use of ICT (Information and Communication Technology) in language learning and the development of new genres or communicative practices such as blogging, web chats, SMS. In many respects, the language learning field was a trailblazer as language professionals saw the potential of various forms of ICT to enhance the language learning process (e.g. CALL, online language learning, video conferencing). Even if the university language teaching environment may not have moved at the same pace as other language learning environments in adopting ICT, there is nevertheless growing evidence of its use in the teaching of languages (e. g. Felix 2001; Levy 2009; Salaberry 2001). In relation to the impact of communication technologies on the shaping of communicative practices, university language curricula show far less evidence of adoption or adaptation. With the exception of language courses specifically geared towards reading in another language (often for research purposes), most university language curricula include a communicative focus. Hence advances in communicative technologies will impact on both modes of language learning as well as on the learning of communicative practices. Although technology-mediated communication between people has not yet replaced face-to-face communication in 'real time and space', there is strong evidence that our modes of communication have not only diversified but also shifted away from more traditional forms of interaction. Of course there are significant differences among language users in terms of accessibility to, as well as uptake of, these modes of communication. For adolescents and young adults, the future and primary 'clients' of university courses, there is mounting evidence that they not only engage to a high degree in technology-mediated interaction but also often prefer this mode of communication for both learning and social purposes. Many of these new modes of communication demand advanced keyboarding skills foregrounding the writing mode. In the context of second and foreign language learning this is a very interesting development as more recent language learning methodologies and approaches (e.g. audio-visual, communicative language teaching) have foregrounded speaking skills. Whilst 
university language classes have not ignored the skills of reading and writing, the teaching and practicing of these skills have not received the same attention as they did during the era of the grammar-translation method. Furthermore, secondary school LL curricula whose orientation is predominantly communicative focus primarily on oral skills development. Consequently, language students often bring less knowledge of reading and writing to the university language classroom. On the other hand, their use of social media - in their dominant language(s) - centres on written use of language through SMS, blogs, twitter, and other forms of online communication. A cursory inspection of university language syllabi revealed considerable variation among languages with regard to the incorporation of new modes of communication and communicative practices: syllabi of German, French, Italian and other main European languages were more likely to choose the text type "email" through which to demonstrate informal writing than those of Polish, Russian, Arabic or Japanese. When it comes to the syllabi of many less widely taught languages, there is little if any evidence of the inclusion of new genres or modes of communication. This observation is symptomatic of a widening gap between research and practice in this area, affecting in particular the learning of languages seen as less central to the global marketplace.

\subsection{Globalisation and the teaching of languages}

The previous sections have outlined how globalisation and its concomitant factors can or will have an impact on the learning of languages. In this section, I will explore the preparedness of university language teachers to deal with the changes in student profiles and communicative practices resulting from globalisation. My focus on university language teachers is directly related to their key role in transforming language teaching and learning. As mentioned before, the university is a prime site for generating as well as transmitting new knowledge and ideas. University language teachers are either themselves involved in research or are close to researchers investigating the new challenges. They could therefore be expected to be at the forefront in applying new insights to the teaching of languages. In 2008, I started a research project in Australia exploring the extent to which university language teachers were aware of the challenges of globalisation to the learning of languages and to what extent they accommodated these in their teaching. When moving to the United Kingdom in 2010 , I continued the research. In the following sections I provide an overview of the findings from interviews and interactions with sixty-two university language teachers. A more detailed report is in preparation (Pauwels forthcoming). 


\subsubsection{Language teacher profiles}

Sixty-two university language teachers were interviewed in Australia (42) and the United Kingdom (20). Both countries are characterised by a high degree of linguistic diversity with regard to their population. Yet English dominates public life. Their higher education systems share many common features. Furthermore, the availability of languages, of LL as well as the position of languages in universities, also displays considerable similarities.

Among them they taught 16 different languages including major European and Asian languages (French, German, Spanish, Italian as well as Chinese, Japanese and Arabic) and a range of less widely taught languages from these regions $^{1}$.

Their disciplinary and professional profile was very much in line with that of fellow language teachers in Australia and the United Kingdom: they included academics (23), language teaching fellows and assistants (27) and postgraduate students (12). Only 8 of these teachers had undertaken university-level studies focussing on language pedagogy, language acquisition or applied linguistics. The majority of the teachers (48) were trained in literary and cultural studies and had had no real exposure to knowledge about language pedagogy. A further 8 language teachers had no training whatsoever: being native speakers of the language, they had been hired without the need to demonstrate language teaching credentials. The latter group were more likely to be involved in the less widely taught languages where the supply of qualified teaching staff is scarce. With the exception of the postgraduate students, the teachers had usually more than ten years' teaching experience with about a third having 25 years' experience in language teaching at university.

In terms of their linguistic profile, 22 teachers had gained proficiency in the target language primarily through formal study and 40 teachers through various forms of home use, either in a diasporic setting (17) or in the 'homeland' (23). The latter group is normally labelled 'native speakers' and those who used the language in a diasporic setting 'heritage language speakers'. The majority of these teachers (47) had some proficiency in an additional language (i. e. other than the language of instruction, English, and the target language): they had gained proficiency in that additional language either through formal study (26) or through some form of home or community use (21).

Although this group of teachers is not statistically representative of language teachers in these settings, their professional and linguistic profile is (e.g.

\footnotetext{
${ }^{1}$ In order to maintain confidentiality of the teachers it is not possible to provide profiles of teachers by language. In most cases there were only one or two interviewees representing less widely taught languages.
} 
Klapper 2001; Nicholas et al.1993). Therefore the views expressed by this group are not likely to be atypical of their colleagues in these countries.

\subsubsection{Teachers' awareness of the impact of globalisation on LL}

Through interviews I explored the themes of language learners and new communication technologies and asked the following questions that acted as conversation starters:

- Can you tell me something about the linguistic profile of your language students?

- How does the presence of plurilingual students affect the teaching and learning process? This refers to students who are 'native' speakers, community/heritage language speakers as well as students who have proficiency in other languages.

- To what extent do the new communication technologies impact or affect your teaching of the target?

Knowledge of the linguistic profile (1)

Language classes are relatively small so that there is ample opportunity for language teachers to get to know their students quite well. Furthermore, students are often asked to provide information about their linguistic profile so that they can be placed in the appropriate level or class. Despite this closer contact with students, it seemed that few teachers knew much about the linguistic profile of their students and usually 'guessed' the latter's profile. In fact, many teachers (22) indicated that they did not really know and that they did not consider it was relevant to their teaching, except if the student was a 'native speaker'. About half of the teachers (33) guessed that their students were predominantly monolingual. Only a minority thought that their students had some preuniversity language experience (other than school-based) such as living in another country or through home exposure. Teachers of less widely taught languages seemed slightly better informed than the others. The main impression I gained from their answers is one of both lack of knowledge about and a disinterest in the linguistic profiles of students, except where the student displayed signs of native speaker knowledge or of being a heritage language speaker. These two categories received a mention and were followed up with comments and remarks linked to the second question.

Presence of plurilingual learners in the class (2)

Their comments about the presence of plurilingual students and their impact on the teaching were also often bland and unrevealing. Those who had shown little 
knowledge or interest in their students' linguistic profile showed a similar indifference to the second question, irrespective of the type of plurilingualism involved. Their comments ranged from 'there's no real effect, some are good and others not', 'it does not seem to matter', 'not sure, I have not really taken any notice' and 'Actually I don't think it makes a difference, I have never heard a student make a comment about it'. Thirty-five teachers provided some comments that can be broadly categorised as follows:

- Such students usually have a negative effect on the class (23);

- Such students have greater metalinguistic awareness about language, which helps them (8);

- Such students seem to have better learning strategies (4).

Since most teachers assumed that their students had a predominantly monolingual profile, their answers have to be understood as quite speculative. Yet they may reveal underlying attitudes towards plurilingual students. Overall, these comments attest to attitudes that are predominantly negative. These students are seen as upsetting class dynamics, as having a level of arrogance with regard to learning a target language, or as taking language classes because it is an easy option. For example, a British interviewee notes "they think they know how to learn and don't pay attention to details", and a teacher from an Australian university mentions "I don't have positive experiences with such learners, especially if their knowledge has come about through home use or informal learning - they have picked up bad habits which are hard to undo". Only a small number of comments presented positive effects of a student's plurilingual profile or prior language experiences. Comments included "I speak perhaps more from my own experience but there is definitely a link, you draw upon the experiences of your other languages to work out, say, the word order", "Of course it makes a difference, you really acquire a range of strategies of how to learn languages" and "these students know how to learn a language, I feel that they are far less anxious and seem to be more relaxed in their approach". There was no real evidence of a correlation between the teachers' professional and linguistic profile and their views on this issue, except in the case of the positive comments: they came almost exclusively from those language teachers who had had exposure or training in language pedagogy or second language acquisition.

\section{Impact of new communication technologies (3)}

This question generated more varied and expansive responses, although most of them focussed on the use of ICT in the delivery of teaching rather than on their impact on shaping new communicative practices. Thirty-eight teachers mentioned that they actively encourage the use of various ICT practices to their students and 20 said that they make use of them in their teaching. Comments 
included "I tell my students to join chat rooms that will put them in touch with native speakers" (German teacher), "I have compiled a list of sites for my students" (French), "In fact my students, at least the really motivated ones, already know how to use these and they tell others in the class" (Italian), "I think it's very good for more advanced users, I find the sites and then tell my students" (Indonesian). Of course not everyone is enthusiastic about or uses ICT, with some complaining that there is little for their language: "I don't think there is much for [African language] but if there was I am not sure I would use it" (African language), "What I have seen is not suitable for learners, it's corrupted language, badly written and I don't want students to develop bad habits" (Middle Eastern language), "If someone else does it, I would be happy to use it but don't really have the time or skill to do it myself" (South Asian language).

These quotes demonstrate that the teachers' awareness of ICT is primarily linked to its use as a teaching/learning tool in the classroom. Those who integrated ICT in their teaching mentioned various technologies and programs including for character writing (Japanese and Chinese), on line grammar and vocabulary tests, second life, video and audio files and satellite TV. Not surprisingly, teachers of the 'bigger' languages (mostly European) were greater users, largely because so many more resources were readily available. In the case of less widely taught languages, the teachers expressed their disappointment at the limited resources.

Particularly noteworthy in response to the third question is the almost complete absence of references to the impact of ICT on communicative practices and genres. Only five interviewees (all teaching European languages) touched upon this aspect briefly. Their comments concerned the text type 'email' and two mentioned telling their students about some conventions in the use of SMS in French. After more specific prompting on my part, interviewees admitted that they were not really sure about this or that they had not given it much thought.

This small-scale study reveals a teaching body that seems to be rather illprepared to tackle the challenges of globalisation to LL. This seems to result from a combination of limited understanding and little interest in the matter. This in turn may well be the result of the disparate nature of language teaching personnel. In this sample, which is not unrepresentative of teaching personnel in universities, at least in Australia and Great Britain, the majority of teachers (48) were trained in literary and cultural studies and saw language teaching as peripheral to their main tasks. The more senior or established academics in this group often commented, sometimes apologetically, that they were neither qualified nor interested in language pedagogical matters and they ended up teaching some language classes because of personnel shortages or because they 
were native speakers of the language. The lack of interest by such academics in language pedagogy is further fuelled by the low status assigned to language teaching in the university setting (i. e. vis-à-vis the teaching of literary, cultural or linguistic aspects associated with the language). For those teaching less widely taught languages there are a number of additional issues that may limit their engagement with these matters: many of them are part-time teachers sometimes employed on minimal fractions (.1 or .2) with limited if any career advancing prospects. This fact combined with a dearth of appropriate teaching resources for their languages provides little incentive to them to go the "extra mile' in adapting their teaching to meet the challenges of globalisation. The comments and reactions from the teachers in this study point towards the widespread use of the 'apprentice' model of teaching, i. e. teachers model their teaching almost exclusively on how they were taught. Klapper (2001: 19) noted that this was quite widespread in British higher education, where there is limited formal training in language teaching and where support for language teachers is also limited: "teachers, [...] are likely to revert uncritically, as a sort of default, to classroom approaches they themselves experienced as learners". In this context it is therefore not surprising that there is limited awareness and uptake of research findings linked to changes in LL. The likelihood of university language teaching being research-informed is low and seems restricted to those teachers whose disciplinary orientation is LL, language pedagogy or applied linguistics.

This state of affairs, if representative of university language teaching globally, is not only disappointing but may well have further adverse effects on the uptake of LL as well as on the perceived value of such learning by 'employers' in preparing graduates for a globalised world.

\section{$3 \quad$ Next Steps?}

Globalisation presents both challenges and opportunities to the field of language learning. It has an impact on the constellations of languages that are learned or are on offer to be learned. Subsequently, languages that are seen to represent desirable linguistic capital in the context of globalisation are preferred and foregrounded leading to (sometimes) considerable shifts in the language learning scene of a country or a community. Despite the heightened multilingualism, even hyperlingualism resulting from the mobility factor linked to globalisation, many of the languages that make up this multilingual landscape are not gaining ground in the learning scene. Yet there is evidence of increasing demands for competencies in some of these languages linked to security, social welfare and trade. Research into factors and elements that affect the linguistic and communicative practices in the context of globalisation is well-advanced, judging by the phenomenal output in research dealing with multilingualism and language learning. This chapter provided a summary insight into some of the 
research themes and explorations linked to language and globalisation: heightened linguistic diversity leading to extensive multilingualism, even hyperlingualism; the commodification of languages putting a market value on language and seeing it as capital, and the impact of communication technologies on language and communicative practices. The results of research into these themes, however, do not seem to filter through to practice. This seems to be linked to the disconnect between language researchers and those teaching the language as mentioned in 2.5.2. The latter group includes only a small proportion of people with direct links to relevant research or relevant formal training. Although this disconnect is also observed in some other fields (e. g. medicine, music), it is particularly prominent in languages, often due to financial woes affecting language departments (at least in Anglophone countries). This is a state of affairs that is likely to continue for many years to come.

Overcoming this disconnect so that research findings can inform teaching requires actions at the level of policy and practice. At a macro-policy level it is pleasing to see that in Europe, the European Commission in collaboration with the Council of Europe, have taken steps to address the issue of language training for teachers (Kelly et al. 2002): this report provides a programme for language teacher training in Europe recommending a benchmark for training, an accreditation framework and a support network. The main emphasis, however, is on the secondary and primary sector with limited input from, or recommendations for, the tertiary sector. Even if this report had been more focused on universities, it is still a voluntary code that universities in Europe would need to be willing to accept. To date there is limited evidence that European universities are willing to impose or implement this framework. At national level (e. g. the United Kingdom, Australia) there are some initiatives for providing training for university teachers: this training is generic rather than discipline-specific and is voluntary rather than compulsory, especially for established academics. Whilst super-regional and national frameworks for language teacher training may prove to be an incentive for universities, the decision to implement such training ultimately lies with the university itself to implement such training. Here is an important role for Heads of Language Departments to convince their colleagues as well as the senior leadership of the university to adopt such training. Professional language organisations also play a crucial role in this process by acting as facilitators for the spreading of good practice in relation to this matter. The national bodies and agencies for quality assurance in higher education and agencies are also central in this process: certification of quality of an institution could be subject to evidence of relevant staff having undertaken such training. Although I believe that 'cracking' the policy level within higher education is key to the successful implementation of training, actions at the level of the classroom are also needed to make staff and students aware of the changed environment. Here again, the role of senior staff 
willing to champion alternative approaches and facilitating their colleagues to access the vast resources available through applied linguistics and language education research is critical. In the British context, small inroads have been made in this direction through the establishment of consortia around languagebased area studies (covering some Asian/Eastern European and Middle Eastern languages). In these consortia, universities work together to enhance language learning experiences for their students. As a result, more language teaching staff come together across institutions sharing practices in ICT and teaching approaches. Undoubtedly, such initiatives are also found in other higher education systems around the world and are more easily shared through the online world. Finally, it is most likely that language students themselves will demand changes: they have become more vocal and more articulate in expressing their views on teaching at university, including language teaching. If they find that their language learning experiences, communicative practices and needs are not reflected in language teaching at universities, or do not help them with the demands of a globalised work place, they will actively seek change or abandon language learning at universities.

\section{References}

Block, David / Cameron, Deborah (eds.) (2002). Globalization and language teaching. London: Routledge.

Blommaert, Jan (2010). The sociolinguistics of globalization. Cambridge: Cambridge University Press.

Cameron, Deborah (1995). Verbal Hygiene. London/New York: Routledge.

Cameron, Deborah (2002). Globalization and the teaching of communication skills. In: Block, David / Cameron, Deborah (eds.). 67-82.

Dörnyei, Zoltàn (2001). Motivational strategies in the language classroom. Cambridge: Cambridge University Press.

Dörnyei, Zoltàn (ed.) (2003). Attitudes, orientations and motivations in language learning. Oxford: Blackwell.

Duchêne, Alexandre / Heller, Monica (eds.) (2012). Language in late capitalism: Pride and profit. London/New York: Routledge.

Felix, Uschi (ed.) (2001). Beyond babel: language learning online. Melbourne: Language Australia.

Fenoulhet, Jane / Ros i Solé, Cristina (2012) (eds.): Mobility and localisation in language learning. Bern: Lang.

Fenoulhet, Jane / Ros i Solé, Cristina (2012). Introduction. In: Fenoulhet, Jane / Ros i Solé, Cristina (eds.). 3-27.

Gardner, Robert / Lambert, Wallace (1972). Attitudes and motivation in second language learning. Rowley, MA: Newbury House. 
Gardner, Robert (1985). Social psychology and language learning: The role of attitudes and motivation. London: Edward Arnold.

Graddol, David (1997). The future of English. London: The British Council.

Graddol, David (2006). English next. London: The British Council.

Gorter, Durk (ed.) (2006). Linguistic landscape: A new approach to multilingualism. Clevedon: Multilingual Matters.

Heller, Monica (2003). Plurilinguisme et didactique. Textes introductifs. In: Séminaire Langues, cultures, identités en didactique des langues. 27-28 mars 2003. Paris: INALCO.

$\mathrm{Hu}$, Guangwei W. (2005). English language teaching in China: Policies, progress, and problems. Language Policy 4: 5-24.

Kelly, Michael / Grenfell, Michael / Gallagher-Brett, Angela / Jones, Diana et al. (2002). The training of teachers of foreign language: Developments in Europe. Brussels: European Commission.

Klapper, John (ed.) (2001). Teaching languages in higher education: Issues in training and continuing professional development. London: CILT.

Kramsch, Claire (2006). Teaching local languages in global settings: The European challenge. Fremdsprachen Lehren und Lernen 35: 1-10.

Levy, Mike (2009). Technologies in use for second language learning. The Modern Language Journal 93: 760-782.

Nicholas, Howard / Moore, Helen / Clyne, Michael / Pauwels, Anne (1993). Languages at the crossroad. Melbourne: The National Languages and Literacy Institute of Australia.

Pauwels, Anne (2007). Maintaining a language other than English through higher education in Australia. In: Pauwels, Anne / Winter, Joanne / Lo Bianco, Joseph (eds.): Maintaining minority languages in transnational contexts. Basingstoke: Palgrave Macmillan. 107-123.

Pauwels, Anne (forthcoming). Teaching languages in the era of globalization.

Salaberry, M. Raphael (2001). The use of technology for second language learning and teaching: A retrospective. The Modern Language Journal 8/1: 39-56.

Zarate, Geneviève (2010). Language biography and international mobility: On the position of multilingual and multicultural capital in the academy. In: Jane Fenoulhet / Ros i Solé, Cristina (eds.). 29-49. 


\title{
Le plurilinguisme est-il un objectif européen pour l'Allemagne?
}

\section{Christiane Fäcke (Augsburg)}

\begin{abstract}
Starting with a presentation of language policy in the European Union, this article presents an analysis of language policy in Germany, that is to say the different federal policies on foreign language education in the school system. The paper then focuses on the mutual influences between language policy in Europe and in Germany as well as their consequences on the theory and practice of language teaching. The approach is characterized by a hermeneutic methodology in the form of an analysis of different administrative documents and by referring to certain recent empirical studies.

Partant d'une présentation de la politique linguistique de l'Union Européenne, cet article présente une analyse de celle de l'Allemagne en soulignant les différentes politiques fédérales de l'enseignement des langues étrangères dans le système scolaire. La contribution traite ensuite des influences mutuelles entre la politique linguistique de l'Union Européenne et de l'Allemagne et de leurs conséquences sur la théorie et la pratique de l'enseignement des langues. L'approche est caractérisée par une méthodologie herméneutique sous forme d'analyse de différents documents administratifs et par un recours à certaines études empiriques récemment réalisées.
\end{abstract}

\section{La politique linguistique de l'Union Européenne}

L'Union Européenne, cette entité de vingt-huit Etats se définissant par différentes cultures, différents mœurs, différentes convictions et différentes langues s'est dotée en 2000 de la devise «Unie dans la diversité ». En promouvant cette diversité, les valeurs transmises sont celles de la solidarité et de la compréhension mutuelle. Cette devise résume la position linguistique de la Commission Européenne (Commission des Communautés Européennes 2003, 2005) pour favoriser le plurilinguisme en Europe, une décision qui se positionne contre l'existence d'une seule langue officielle - la lingua franca - qui sans conteste est l'anglais.

Cette politique se rapporte à la réalité linguistique en Europe. Alors que le plurilinguisme est considéré comme un fait accompli dans beaucoup de pays du monde entier (cf. Klein 2002: 34), il l'est beaucoup moins en Europe. De plus, il y a souvent un décalage entre un plurilinguisme individuel dû à l'existence de plusieurs dialectes et de langues minoritaires autochtones et allochtones d'un côté, et une politique nationale basée sur une seule langue officielle désignée à représenter l'identité nationale de l'autre (Cichon 2008: 21). Le plurilinguisme existe dans des pays comme le Luxembourg et les Pays-Bas avec un pourcentage de plus de $70 \%$ des habitants parlant plus d'une langue. Par ailleurs, en Hongrie et en Grande-Bretagne, seule 35\% à 40\% de la population disposent de connaissances dans une deuxième langue (Commission Européenne 2012: 17). 
Les connaissances en langues étrangères ne dépendent certes pas d'un talent individuel ou collectif de la population d'un pays, mais plutôt du nombre des locuteurs d'une langue ou encore de ses représentations sociales. Les membres d'une petite communauté linguistique se voient beaucoup plus dans l'obligation d'apprendre des langues étrangères que les membres d'une communauté linguistique dont la langue est la lingua franca ou bien langue de communication internationale. Tandis que les Britanniques constatent que l'anglais est parlé dans la majorité des pays, il en va autrement pour les Luxembourgeois et la langue luxembourgeoise. En général, les compétences linguistiques sont assez étendues dans les états qui disposent de plusieurs langues officielles (Luxembourg) ou dont la langue la plus représentative est globalement relativement peu utilisée (Les Pays-Bas). Ainsi

[...], les compétences linguistiques semblent être légèrement meilleures dans les Etats membres plutôt petits, comme le Luxembourg, les Pays-Bas et la Slovénie, tandis que les citoyens de l'Europe méridionale et des deux pays où l'on parle anglais, c'est-à-dire le Royaume-Uni et l'Irlande, semblent avoir des connaissances linguistiques plutôt modérées. (Commission Européenne 2006: 11)

Ce contexte européen permet de mieux comprendre l'objectif linguistique et politique de la Commission Européenne de favoriser le plurilinguisme (Fäcke 2008).

\section{La politique des langues étrangères en Allemagne}

Comment l'Allemagne réagit-elle face à ces objectifs de l'Union Européenne en faveur du plurilinguisme, alors que l'allemand est la langue qui bénéficie du plus grand nombre de locuteurs au sein de l'Union Européenne? Considérons la position de la conférence des ministres de l'Éducation et des Affaires Culturelles d'un Land ${ }^{1}$, les programmes scolaires de quelques Länder, le choix des langues enseignées dans les établissements scolaires et le nombre d'élèves apprenant une ou plusieurs langues.

Cette focalisation sur les dimensions politiques et sur l'enseignement des langues étrangères dans le système scolaire allemand est dû au fait que l'Allemagne soutient cette forme de plurilinguisme dans une certaine mesure, négligeant le plurilinguisme individuel pratiqué par un nombre considérable de minorités ethniques. La prise en compte de l'apprentissage des langues d'origine a longtemps été considérée comme un moyen pour faciliter la réinsertion des migrants dans leurs pays d'origine respectifs. Ces langues d'origine n'ont jusqu'à ce jour pas encore de place affirmée dans l'enseignement des langues étrangères du système scolaire.

\footnotetext{
${ }^{1}$ Kultusministerkonferenz.
} 
La conférence des ministres de l'Education et des Affaires Culturelles d'un Land étant composée de tous les ministres des différents Länder allemands lesquels déterminent eux-mêmes leur politique éducative, il est alors indispensable de discuter les différentes décisions politiques des Länder afin de les harmoniser jusqu'à un certain point. Cette conférence a trouvé une position commune dans les standards éducatifs ${ }^{2}$ en s'appuyant sur le plurilinguisme comme réalité sociale:

Domaines de compétence de la première langue étrangère (anglais/français) Le plurilinguisme constitue une réalité dans bien des domaines de notre société, cependant dans des qualités assez différentes. Or, il est à attendre de l'enseignement de la première langue scolaire que les compétences communicatives, interculturelles et méthodiques des élèves soient développées afin d'agir dans des situations plurilingues à la fin de la Sekundarstufe I [correspondant à la fin du collège]. (Kultusministerkonferenz 2003: 11) ${ }^{3}$

Compte tenu du système fédéral allemand, chaque Land dispose d'une autonomie au niveau de la politique culturelle et scolaire. Cette autonomie se reflète dans les programmes scolaires, notamment dans des différences qui se manifestent en comparant, par exemple, la Bavière et la Hesse.

En Bavière, le programme scolaire cite le plurilinguisme recommandé par l'Union Européenne en référence à l'anglais, au français, à l'italien, au russe et à l'espagnol.

En cours de français, les élèves acquièrent des connaissances liées à l'application de la langue française contemporaine pour la vie quotidienne, leurs études et le travail. [...] En outre, ils améliorent leur culture générale par le fait qu'ils [...] acquièrent, au lycée, le niveau requis de sensibilisation à la langue et, dans le sens de l'apprentissage continu et du plurilinguisme exigé par l'UE, et qu'ils accèdent à l'apprentissage d'autres langues internationales telles que l'espagnol après leur scolarité. (Bayerisches Staatsministerium 2010: 22)

La Bavière mentionne explicitement dans ses programmes les objectifs européens concernant le plurilinguisme. Cependant, si susciter l'intérêt pour les langues étrangères fait partie des objectifs scolaires, leur accès réel est plutôt

\footnotetext{
${ }^{2}$ Bildungsstandards.

${ }^{3}$ [Kompetenzbereiche der ersten Fremdsprache (Englisch/Französisch)]: Mehrsprachigkeit stellt für nicht wenige Teilbereiche unserer Gesellschaft eine Realität dar, allerdings in unterschiedlichster qualitativer Ausprägung. Vom Fremdsprachenunterricht in der ersten Schulfremdsprache ist daher zu erwarten, dass die kommunikativen, interkulturellen und methodischen Kompetenzen der Schülerinnen und Schüler für ihr Handeln in mehrsprachigen Situationen am Ende der Sekundarstufe I verlässlich ausgebildet worden sind. (Kultusministerkonferenz 2003: 11). (Je remercie Sylvie Méron-Minuth qui a fait les traductions de tous les textes officiels.)
} 
encouragé à la fin des études scolaires. De plus, une politique prenant en compte les langues d'origine n'est nullement prise en considération.

Le programme scolaire de la Hesse mentionne également le plurilinguisme:

Les contenus fondamentaux pour le français langue étrangère en tant que discipline scolaire dans les classes 5 à 12 du lycée l: $^{4}$ ) Tâches et objectifs [:] En raison de l'agrandissement continu de l'Union Européenne et de l'internationalisation de tous les domaines du quotidien, le plurilinguisme des citoyens de l'Union Européenne est essentiel pour la libre circulation de la vie professionnelle et personnelle. [...] L'éducation scolaire en Hesse, notamment l'éducation au lycée, se doit de s'ouvrir à ce développement dynamique ainsi qu'à la demande de réalisation du plurilinguisme des citoyens européens afin de pouvoir se maintenir durablement dans la compétition internationale. (Hessisches Kultusministerium 2008: 3 ) $^{5}$

La Hesse, comparée à la Bavière, souligne explicitement le plurilinguisme et s'oblige à contribuer à sa réalisation à travers l'éducation, surtout dans le second degré (lycée). Cependant, l'argumentation de ce texte est d'utiliser le plurilinguisme pour justifier l'apprentissage du français dans les lycées de la Hesse. Un plurilinguisme qui prendrait en compte d'autres langues d'origine tel le turc ou l'arabe, n'y est pas mentionné, or il s'agit d'une conception favorisant certaines langues au détriment d'autres.

L'adhésion au plurilinguisme et le plaidoyer pour sa réalisation dans les établissements scolaires sont documentés par les deux textes administratifs précédents, même si malgré tout des nuances persistent quant à l'importance de cette adhésion. Ces idées invitent à examiner de plus près la réalité scolaire dans les différents Länder et à élucider la question de savoir si ces déclarations représentent une véritable décision politique ou bien si elles relèvent davantage des discours artificiels. Les statistiques nous renseignent sur les dimensions du plurilinguisme à travers le nombre d'élèves apprenant une langue étrangère en Allemagne. Dans une perspective comparative, tableau 1 montre le développement quantitatif entre les années 2007/2008 et 2011/2012.

\footnotetext{
${ }^{4}$ Les classes 5 et 12 du lycée en Allemagne correspondent à la sixième et à la terminale en France.

${ }^{5}$ Grundlegung für das Unterrichtsfach Französisch in den Jahrgangsstufen 5G bis 12G: 1) Aufgaben und Ziele des Faches[:] Durch die immer weiter fortschreitende Erweiterung der Europäischen Union und der Internationalisierung des Lebens in allen Bereichen wird die Mehrsprachigkeit der Bürger der Europäischen Union unerlässlich für die Freizügigkeit im beruflichen und privaten Bereich. [...] Auf diese dynamische Entwicklung und die damit verbundene Forderung nach Mehrsprachigkeit der europäischen Bürgerinnen und Bürger muss sich schulische Bildung in Hessen, insbesondere gymnasiale Bildung, einlassen, um im internationalen Wettbewerb weiterhin bestehen zu können. (Hessisches Kultusministerium 2008: 3)
} 
Le plurilinguisme est-il un objectif européen pour l'Allemagne?

Tableau 1: L'enseignement général. Sources: Statistisches Bundesamt Deutschland (2012 : $90 \mathrm{a})^{6}$

\begin{tabular}{|l|l|c|l|}
\hline \multirow{2}{*}{ Langues } & \multicolumn{3}{|c|}{ Élèves inscrits dans l'enseignement des langues } \\
& \multicolumn{3}{|c|}{ Années scolaires } \\
\cline { 2 - 4 } & $2007 / 08$ & $2011 / 2012$ & \\
\cline { 2 - 4 } & Effectifs & Effectifs & CA $^{7}$ en \% \\
\hline Anglais & 7372865 & 7551732 & $+2,4$ \\
\hline Français & 1696411 & 1632803 & $-3,8$ \\
\hline Latin & 825275 & 772705 & $-6,4$ \\
\hline Grec (ancien) & 15909 & 14000 & $-12,0$ \\
\hline Espagnol & 285480 & 347664 & $+21,8$ \\
\hline Italien & 52111 & 58020 & $+11,3$ \\
\hline Russe & 99991 & 106620 & $+6,6$ \\
\hline Turc & 10977 & 10011 & $-8,8$ \\
\hline autres langues & 54787 & 65648 & $+19,8$ \\
\hline
\end{tabular}

En termes de nombre d'apprenants de langues étrangères, l'anglais est largement prédominant, suivi par le français et le latin. Bien qu'en chiffres absolus l'espagnol se trouve à un niveau incontestablement inférieur, le nombre d'élèves augmente régulièrement comparé aux autres langues. L'enseignement des langues d'origine, en chiffres absolus, ne semblerait avoir que très peu d'importance dans le système scolaire allemand. On trouve davantage d'élèves apprenant le grec ancien, langue morte, que d'élèves apprenant le turc, langue pratiquée par une des plus anciennes et plus importantes minorités en Allemagne. Le bilinguisme des élèves turcs-allemands n'est pas mentionné dans ces statistiques. Ni l'allemand langue étrangère ni les langues d'origine ne

\footnotetext{
${ }^{6}$ http://www.destatis.de/jetspeed/portal/cms/Sites/destatis/Internet/DE/Content/Statistiken/ BildungForschungKultur/Schulen/Tabellen/Content50/AllgemeinbildendeSchulen FremdsprachUnterricht.psml.

${ }^{7}$ Changement par rapport aux chiffres précédents.
} 
jouent un rôle important. Ceci montre de manière implicite que le plurilinguisme des minorités ethniques en Allemagne reste peu valorisé bien qu'il y ait de plus en plus de programmes de soutien pour l'amélioration de la langue d'origine. Si l'Allemagne souhaitait adhérer aux exigences et objectifs européens de réaliser un plurilinguisme basé sur des connaissances dans trois langues au minimum la langue maternelle plus deux autres langues étrangères (Commission européenne 1995: 47) - il conviendrait ainsi de se concentrer sur l'enseignement de la deuxième langue la plus étudiée, c'est-à-dire le français ou encore de porter son attention sur l'enseignement d'une langue d'origine - l'anglais ayant acquis depuis longtemps une position maitresse.

Ces statistiques présentent encore d'autres chiffres concernant le français. En 2010/11, 1,65 millions d'élèves ont appris le français, ce qui représente un pourcentage de $19 \%$, chiffre relativement stable depuis 20 ans tout comme celui de l'anglais (87\%) et du latin (9,0\%). Dans le secteur de l'enseignement primaire, une très grande majorité d'élèves apprend l'anglais. La plupart des élèves de 11 à 16 ans se trouve au lycée $(34,4 \%)$ et moins dans les autres secteurs de l'enseignement scolaire en Allemagne (Realschule: 26,4\%, Integrierte Gesamtschule: 11,0\%, Schularten mit mehreren Bildungsgängen ${ }^{8}$ : $8,4 \%$ ). En général, le français est enseigné comme deuxième langue vivante (Statistisches Bundesamt 2012b).

${ }^{8}$ Les types d'écoles mentionnés ici représentent différentes filières du premier et du second cycle en Allemagne qui correspondent au collège. 
Cependant, le nombre d'élèves participant à l'enseignement du français varie selon les Länder:

Tableau 2: Enseignement général, enseignement du français dans les établissements scolaires selon les Länder (Statistisches Bundesamt 2012b)

\begin{tabular}{|l|l|}
\hline Land & $\begin{array}{l}\text { Pourcentage dans l'enseignement du français par } \\
\text { rapport au total des élèves }\end{array}$ \\
\hline Bade-Wurtemberg & 26 \\
\hline Bavière & 12,4 \\
\hline Berlin & 17,8 \\
\hline Brandebourg & 18,7 \\
\hline Brême & 12,4 \\
\hline Hambourg & 15,4 \\
\hline Hesse & 22,2 \\
\hline Mecklembourg-Pomér-Occi. & 12,4 \\
\hline Basse-Saxe & 14,0 \\
\hline Rhénanie-du-Nord-Westph. & 18,4 \\
\hline Rhénanie-Palatinat & 24,0 \\
\hline Sarre & 58,0 \\
\hline Saxe & 15,7 \\
\hline Saxe-Anhalt & 12,9 \\
\hline Schleswig-Holstein & 18,2 \\
\hline Thuringe & 23,9 \\
\hline Allemagne & 18,8 \\
\hline
\end{tabular}

Le chiffre assez faible en Bavière s'explique, entre autres, par les restrictions du choix des langues étrangères et par l'ordre chronologique de l'apprentissage des langues. En Bavière, les élèves sont contraints de choisir entre l'anglais et le latin comme première langue étrangère, entre le latin et le français comme deuxième langue suivi par l'espagnol et l'italien comme troisième ou quatrième 
langue. Ceci montre une dominance nette du latin qui supplante le français dans ce Land.

D'autres Länder présentent un choix plus étoffé de langues étrangères que la Bavière. A Berlin par exemple, il est possible d'apprendre l'espagnol, le français, le grec, l'italien, le polonais, le portugais, le russe, le turc ainsi que le japonais et le chinois. Les langues anciennes telles que le latin et le grec sont également enseignées. Généralement, l'anglais est enseigné comme première langue, néanmoins il reste possible de choisir différentes langues dans les écoles primaires et secondaires. De plus, il existe un enseignement bilingue allemandanglais ou allemand-français, mais également allemand-espagnol, allemanditalien, allemand-grec (moderne), allemand-turc ou encore allemand-russe, pour ne nommer ici que quelques exemples (Senatsverwaltung für Bildung, Wissenschaft und Forschung ${ }^{9}$ 2009).

Cette comparaison des données statistiques souligne bien les différentes politiques linguistiques au sein de l'Allemagne en fonction des Länder. La structure fédérale se reflète dans le choix des langues étrangères enseignées dans les écoles des différents Länder. En fonction des traditions scolaires, de la position géographique ou encore de la position dominante de certains partis politiques dans un Land, on peut trouver une variété considérable de langues enseignées dans les écoles (Coste 1998).

Ces statistiques nous informent ainsi sur l'étendue d'un plurilinguisme promu par l'enseignement des langues étrangères dans le système scolaire en Allemagne. En outre, il semble intéressant de déceler une perspective plus individuelle et subjective. Le prestige des langues et les attitudes des élèves visà-vis de celles-ci constituent également des facteurs importants de développement du plurilinguisme.

Les résultats d'une enquête quantitative parmi les étudiants allemands (Meißner/Schröder-Sura 2009) qui analysent de façon rétrospective leurs expériences avec l'enseignement des langues soulignent la dominance indiscutable de l'anglais. Cette langue bénéficie, selon diverses enquêtes européennes, d'une motivation considérable de la part des apprenants, une motivation qui s'explique par le fait que l'anglais soit reconnu comme langue internationale attrayante et dominante dans la culture des jeunes (Meißner/Schröder-Sura 2009: 27). Ainsi, l'anglais ne peut pas être considéré comme une langue étrangère au même titre que les autres langues; elle représente davantage une compétence fondamentale tout comme l'apprentissage de la lecture et de l'écriture.

\footnotetext{
${ }^{9}$ Ministère de l'Éducation et des Sciences de Berlin.
} 
La motivation à apprendre d'autres langues étrangères résulte surtout de la volonté d'acquérir des compétences en communication orale. Cette motivation se voit parfois affaiblie par le fait que les élèves obtiennent souvent de mauvaises notes. Le français reste dans beaucoup d'esprits une langue difficile à apprendre. Les cours de français langue étrangère sont dominés par l'enseignement de la grammaire, la concentration sur les fautes d'orthographe ainsi que le problème des interférences négatives avec l'anglais car les élèves ont tendance à comparer leurs expériences en cours d'anglais avec celles vécues dans les cours d'autres langues étrangères (Meißner/Schröder-Sura 2009).

Ces perspectives quant au plurilinguisme montrent que le choix de langues est influencé par des critères personnels ou sociohistoriques tels les traditions éducatives, l'offre de langues, les politiques linguistiques mais également le prestige des langues et leur influence culturelle sur le marché international.

\section{Perspectives européenne et allemande: interdépendances?}

Quelles conséquences pouvons-nous tirer des faits contextuels énumérés cidessus et de leur imbrication? Quelles influences peut-on constater entre les décisions politiques, la réalité scolaire de l'enseignement des langues étrangères et la didactique des langues en Allemagne?

Les influences de la politique européenne, et plus concrètement les effets du Cadre européen commun de référence pour les langues (CECRL, Conseil de l'Europe 2001) se réalisent au niveau didactique dans le domaine de compétences à atteindre et de tests, au niveau du choix des langues possibles selon les contextes et les politiques linguistiques éducatives des Länder. Le CECRL permet ainsi une autre perspective de la conception de l'enseignement des langues grâce à l'introduction de la notion de compétences (de $\mathrm{A} 1 \mathrm{à} \mathrm{C} 2$ ), à la mise en valeur des compétences des élèves (et non de leurs déficits ou lacunes), à la distinction des savoirs, et à une approche qui souligne une égale importance de toutes les langues. Par conséquent, ces innovations ont invité à questionner la didactique, à orienter les pratiques d'enseignement qui pourraient se concentrer davantage sur les compétences orales, à placer les déficits et les fautes en seconde position et surtout à se référer davantage aux connaissances plurilingues des élèves. Il en résulte au fur et à mesure de nouvelles décisions didactiques, de nouveaux règlements et programmes scolaires (Bildungsstandards), ainsi qu'un développement de nouvelles pratiques de l'enseignement (p. ex. approche par tâches) et par-là même de l'évaluation.

De plus, le CECRL a pour objectif de prendre en compte toutes les langues modernes, c'est-à-dire d'aller à contre-courant d'une certaine hiérarchie des langues enseignées traditionnellement à l'école, et de respecter les langues d'origine traditionnellement oubliées dans cet enseignement. Cependant, nous 
pouvons constater que la politique éducative en Allemagne n'est - pour des raisons surtout financières - pas encore en mesure de réaliser les recommandations du CECRL impliquant l'ensemble des langues modernes. Proposer l'enseignement d'un choix varié de langues étrangères dans les établissements scolaires reste davantage onéreux que de proposer une seule langue. La politique linguistique de certains Länder, notamment de la Bavière, souligne cette résistance.

Si les objectifs du CECRL étaient intégrés au fur et à mesure dans les programmes scolaires et si plus d'importance était accordée non seulement aux compétences orales mais aussi plurilingues, les attitudes des élèves en seraient alors influencées de manière positive. Comme l'enquête de Meißner/SchröderSura (2009) le souligne, c'est surtout l'oral qui motive les élèves dans l'apprentissage d'une langue. Or, accroître l'importance de l'oral dans les programmes scolaires, ce qui est également l'objectif du CECRL, pourrait contribuer à augmenter la motivation et la réussite des élèves à apprendre des langues étrangères. De plus, développer des compétences plurilingues orales pourrait également favoriser non seulement la motivation mais aussi l'efficacité des apprentissages linguistiques.

\section{$4 \quad$ Vers une dynamique de la didactique des langues}

Quelles seraient les répercussions sur une didactique qui ne peut négliger une approche européenne théorique et pratique de l'enseignement des langues? Étant donné que la politique européenne vise à encourager le plurilinguisme ainsi qu'une approche positive de l'enseignement des langues, la didactique se voit contrainte d'en tirer des conséquences et de renouveler son questionnement par des recherches empiriques dans ce domaine. Ainsi, il conviendrait de poursuivre les trois perspectives suivantes:

\section{Concentration sur l'oral}

Dans l'enseignement des langues étrangères en Allemagne, les compétences écrites continuent de jouer un rôle important dans la notation des élèves. Ce fait nécessite un changement car maîtriser une langue étrangère signifie en premier lieu acquérir des compétences orales. Ainsi, la didactique est invitée à développer des approches et des méthodologies qui contribueraient à se concentrer davantage sur l'oral, à placer la compréhension et la production orales au centre de l'enseignement. Il s'agirait donc de développer une formation initiale et continue des enseignants pour les encourager à intégrer ces nouvelles orientations. De surplus, du côté politique on prévoit le remplacement d'un contrôle écrit par un orale. Dans le cadre d'excursions dans un pays francophone, par exemple, il est indispensable pour les élèves de comprendre 
leurs interlocuteurs et de participer à des conversations, et il en est de même lors d'échanges de classe en visioconférences. L'enseignement du français devrait ainsi trouver des solutions pour transmettre ces compétences aux élèves et de les inclure dans la notation.

\section{Nouvelle culture d'évaluation}

Il conviendrait également de changer les processus d'évaluation des compétences. Ces derniers devraient respecter davantage les critères de qualité d'évaluation, telles que l'objectivité, la validité et la fiabilité pour les tests standardisés mais la qualité de l'évaluation passe également par des critères d'éducation d'apprendre à apprendre. Ceci signifie par exemple de développer d'autres formats de tests, un développement qui a déjà été amorcé. La recherche en didactique des langues étrangères se doit de participer de façon plus engagée et active à cette discussion en menant des enquêtes et études empiriques sur toutes les dimensions de l'évaluation.

\section{Introduction de plusieurs langues dans le système scolaire}

Une didactique qui prend en compte le plurilinguisme (Holtzer 2004) devrait repenser les questions suivantes: Comment et jusqu'à quel point pourrait-on inclure plusieurs langues - les langues scolaires comme les langues d'origine des apprenants - dans le système scolaire? Ces questions ont déjà été débattues mais elles ne sont pas encore résolues. Le système scolaire allemand devrait respecter davantage les langues d'origine comme le turc et considérer autant les compétences des élèves turcs face à l'allemand que celles des élèves allemands face à l'anglais. De plus, il serait souhaitable de repenser l'offre des langues proposées à l'école et de rééquilibrer le choix des langues face à l'anglais.

Il résulte que la didactique des langues est invitée à, voire obligée de, réfléchir à l'ensemble de ces questions d'ordre scientifique et pratique, de se positionner face à la politique linguistique européenne en faveur du plurilinguisme, et de développer de nouvelles notions et de nouveaux dispositifs pour son implémentation.

\section{Bibliographie}

Bayrisches Staatsministerium für Unterricht und Kultus (2010). Der Lehrplan für das Gymnasium in Bayern im Überblick. ISB / Staatsinstitut für Schulqualität und Bildungsforschung (ed.). Online: http://www.verwaltung.bayern.de/Anlage3999497/LehrplanGymnasiumBayern Ueberblick2010.pdf (dernier accès [30.07.2013]).

Cichon, Peter (2008). Unterschiedliche Traditionen des sozialen und politischen Umgangs mit Mehrsprachigkeit in Europa. Mehrsprachigkeit für Europa - sprachen- und bildungspolitische Perspektiven. Osnabrücker Beiträge zur Sprachtheorie 74: 21-31. 
Commission des Communautés Européennes (2003). Communication de la Commission au Conseil, au Parlement Européen, au Comité Economique et Social et au Comité des Régions. Promouvoir l'apprentissage des langues et la diversité linguistique: un plan d'action 2004-2006. COM (2003) 449 final. Online: http://ec.europa.eu/education/doc/official /keydoc/actlang/act_lang_fr.pdf (dernier accès [30.07.2013]).

Commission des Communautés Européennes (2005). Communication de la Commission au Conseil, au Parlement Européen, au Comité Economique et Social et au Comité des Régions. Un nouveau cadre stratégique pour le multilinguisme. COM (2005) 596 final. Online: http://ec.europa.eu/education/languages/archive/doc/com596_fr.pdf (dernier accès [30.07.2013]).

Commission européenne (1995). Enseigner et Apprendre: vers une société cognitive. Bruxelles. Online: http://ec.europa.eu/languages/documents/doc409 en.pdf (dernier accès [30.07.2013]).

Commission européenne (2012). Eurobaromètre Spécial 386. Les européens et leurs langues. Rapport. Online: http://ec.europa.eu/public_opinion/archives/ebs/ebs_386_fr.pdf (dernier accès [30.07.2013]).

Conseil de l'Europe (2001). Un Cadre Européen commun de Référence pour les langues. Apprendre, enseigner, évaluer. Paris: Didier.

Coste, Daniel (1998). Quelques remarques sur la diversification des langues en contexte scolaire. In: Billiez, Jacqueline (éd.): De la didactique des langues à la didactique du plurilinguisme. Grenoble: CDL-LIDILEM, Univ. Stendal-Grenoble III. 259-270.

Fäcke, Christiane (2008). Mehrsprachigkeit im Kontext europäischer Sprachenpolitik. In: Frings, Michael / Vetter, Eva (éds.). Mehrsprachigkeit als Schlüsselkompetenz: Theorie und Praxis in Lehr- und Lernkontexten. Akten zur gleichnamigen Sektion des XXX. Deutschen Romanistentages an der Universität Wien (22. bis 27. September 2007). Stuttgart: ibidem. 11-26.

Hessisches Kultusministerium (2008). Lehrplan Französisch. Gymnasialer Bildungsgang. Jahrgangsstufen $5 \mathrm{G}$ bis $12 \mathrm{G}$. Online: http://www.kultusministerium.hessen.de/irj/HKM Internet?uid=3b43019a-8cc6-1811-f3ef-f91921321b2 (dernier accès [30.07.2013]).

Holtzer, Gisèle (2004). Voies vers le plurilinguisme. Besançon: Presses Universitaires de l’Université de Franche-Comté.

Klein, Horst G. (2002). Das Französische: die optimale Brücke zum Leseverstehen romanischer Sprachen. Französisch heute 33/1: 34-46.

Kultusministerkonferenz (2003). Bildungsstandards für die erste Fremdsprache (Englisch / Französisch) für den mittleren Schulabschluss. Beschluss vom 4.12.2003. Online: http://www.kmk.org/schul/Bildungsstandards/1.Fremdsprache_MSA_BS_04-12-2003.pdf (dernier accès [30.07.2013]).

Meißner, Franz-Joseph / Schröder-Sura, Anna (2009). Studierende erinnern sich an ihren schulischen Französischunterricht. Eine quantitative Untersuchung. Französisch heute 40/1: 24-36. 
Senatsverwaltung für Bildung, Wissenschaft und Forschung (2009). Bildung für Berlin. Berlin spricht viele Sprachen. Wegweiser für die Fremdsprachenwahl in der Grundschule und den weiterführenden Schulen. Schuljahr 2009/2010. Online: http://www.berlin.de/imperia/md/content/senbildung/besondere_paedagogische_konzepte/sprachen_lernen/fremds prachen_berliner_schule.pdf?start\&ts $=1234878714 \&$ file $=$ fremdsprachen_berliner_schule. pdf (dernier accès [30.07.2013]).

Statistisches Bundesamt (2012a). Bildung und Kultur. Allgemeinbildende Schulen. Schuljahr 2011/2012. Fachserie 11, Reihe 1. Online: https://www.destatis.de/DE/Publikationen/ Thematisch/BildungForschungKultur/Schulen/AllgemeinbildendeSchulen2110100127004. pdf;jsessionid=B0654152A91AD8A48C31131AE48DD37D.cae2? _blob=publicationFile (dernier accès [30.07.2013]).

Statistisches Bundesamt (2012b). Schulen auf einen Blick. Online: https://www.destatis.de/ DE/Publikationen/Thematisch/BildungForschungKultur/Schulen/BroschuereSchulenBlick 0110018129004.pdf?_blob=publicationFile (dernier accès [08.11.2013]). 



\title{
Language Use by London Bangladeshi and Chinese Adolescents: Some Language Diary Data
}

\author{
Martha C. Pennington / Itesh Sachdev / Lawrence Lau ( $†$ ) \\ (Hong Kong, London)
}

This study examined patterns of language use by London Bangladeshi and Chinese adolescents who are the first UK-born generation of their families, based on self-reports in language diaries. Both adolescent groups reported using English, their heritage language (Bengali for the Bangladeshis and Cantonese for the Chinese), and mixtures of these, depending on the circumstances of the interactions. The Chinese adolescents reported greater use of English than the Bangladeshis, less use of the heritage language, and more mixed language use. In contexts of intimacy, heritage languages and combinations of these with English were reported as common, with Bengali predominating for the Bangladeshis, and mixed (Cantonese-English) language predominating for the Chinese except in speaking to younger relatives, when English was the language of choice. English predominated in the diary data for both groups outside these contexts of intimacy, though the Chinese reported significant mixed language usage in public settings. The findings support a view of these secondgeneration children of immigrants as adopting the main community language, English, while also using their heritage languages.

Cette enquête a examiné les profils d'utilisation de la langue par des adolescents bangladais et chinois de Londres qui sont nés comme la première génération de leur famille au Royaume Uni. Elle se base sur des autodéclarations des adolescents dans des journaux intimes. Les deux groupes ont déclaré qu'ils ont utilisé l'anglais, leur langue d'origine (le bengali pour les bangladais et le cantonais pour les chinois), et les mélanges de ceux-ci, selon les circonstances des interactions. Les adolescents chinois ont signalé une plus grande utilisation de la langue anglaise que les bangladais, moins d'utilisation de la langue d'origine, et plus d'utilisation de langue mixte. Dans les contextes privés défini par l'interlocuteur et la situation, les langues patrimoniales et combinaisons de ces derniers avec l'anglais étaient aussi courantes. Le bengali prédominait pour les bangladais et la langue mixte (cantonais-anglais) prédominait pour les chinois, sauf en s'adressant aux plus jeunes membres de la famille, lorsque l'anglais était la langue optée. D'après les données des journaux intimes, l'anglais était la langue utilisée le plus souvent par les deux groupes. Par contre, l'anglais prédominait dans les données de journal de langue pour les deux groupes en dehors de ces contextes privés. Néanmoins, les chinois ont signalé l'utilisation fréquente de langue mixte en milieu public. Les résultats soutiennent une vue de ces enfants de deuxième génération d'immigrants adoptant la langue de la communauté principale, l'anglais, tout en maintenant la vitalité de leurs langues d'origine. 


\section{$1 \quad$ Introduction $^{1}$}

An important issue in multicultural societies is the degree to which minority ethnic groups acquire and use the majority language while maintaining and also developing their heritage languages and associated cultural and social practices. The research reported here investigates this issue by exploring patterns of linguistic and cultural adaptation by second generation members of two of the largest and most visible, yet under-researched, immigrant communities in London: Bangladeshis and Cantonese-speaking Chinese. The study investigates the use of English and the heritage languages of these two groups, Bengali and Cantonese, through language diaries kept by secondary students who are the first generation of UK-born children in their families. Language use is not examined directly but rather through participants' perceptions of their language choices. This investigation is one in a series of studies using different research methods to investigate the language and identity of London Bangladeshis and Chinese as two ethnically distinctive groups with differing social profiles (see also Pennington et al. 2011; Sachdev/Pennington 2013).

Bangladeshis in London are relatively recent arrivals with only one UK-born generation having reached adulthood. Although the Hong Kong Chinese have a longer history of immigration to London that began in the nineteenth century, this initial wave of Chinese was expulsed before World War II. The current population of Hong Kong origin Chinese in London have an immigration history which is similar to that of the Bangladeshis (i. e., post-World War II), with generally only one UK-born generation having reached adulthood. Members of these groups are also similar in that they both largely began their occupational lives in London by being engaged in the catering trade. They have in common as well the fact that they originate from former outposts of the British Empire. They are, however, different in a number of respects: the Bengali-speaking community is larger and more concentrated in specific areas of London than the Cantonese-speaking community. In addition, the Bangladeshis who have settled in London are of rural rather than urban background and are considerably more disadvantaged than the London Chinese in terms of income, education, and other measures. Given these similarities and differences in background and demographics, both similarities and differences can be expected in the language

1 The research this article is based on was part of the project 'Language and Identity of Bangladeshis and Chinese in London', carried out with the aid of funding in 2004-2006 by a grant (ref. no. RES-000-22-0567) from the UK Economic and Social Research Council (ESRC) to Professors Itesh Sachdev and Martha C. Pennington, with Dr. Lawrence Lau (now deceased) serving as full-time researcher on the project. We gratefully acknowledge the enabling support provided by the ESRC grant for our research. 
use of these two groups, which is explored in a study of members of the second generation from these two migrant communities in London.

For speakers who have a knowledge of more than one language, the choice to use one language or another, or a combination of languages, the way in which the languages are used, such as in various forms of code-mixing, codeswitching, and other creative "translanguaging" (García 2007: xii) modes and the purposes to which individual and multiple languages are put in specific contexts, can all express social orientation and identity (Blackledge/Creese 2008; Sachdev et al. 1977; Sachdev/Bourhis 2005; Sachdev/Giles 2004; Fishman 1980; Le Page/Tabouret-Keller 1985; May 2005; McNamara 1987; Myers-Scotton 1993). Speakers' orientations to and patterns of usage, both formal and functional, of different languages may be an "important and constitutive factor of their individual, and at times, collective identities" (May 2005: 330) signifying their personal and social orientation in both intragroup and intergroup communication (Sachdev/Bourhis 1991, 2001, 2005; Sachdev/Giles 2004) and expressing "their sense of belonging and selfhood" (Blackledge/Creese 2008: 535) as well as their sense of distance and difference (Sachdev/Bourhis 2001; Sachdev et al. 2012). The connection of language use to social orientation and identity is however not straightforward, since neither language nor identity, nor their interrelationships, are static. Rather, they are evolved by speakers for their own purposes. Both the forms and functions of languages, including so-called 'heritage' and 'majority' languages, are continually changed by those who use them (Pennington 2000, forthcoming), and their changing profiles are linked in new and different ways to social functions and meanings - and so to speaker identity as related to those social functions and meanings.

A knowledge of multiple languages provides diverse means for evolving and expressing different facets of identity and aspects of social meaning through the choices speakers make of which language(s) to use and the specific forms and functions of their language usage in different circumstances. Language choice within social or ethnic groups as studied by the language diary method has been found to vary according to factors such as whether the speaker is talking to family members, friends, or others, whether the talk takes place in a private or a public space and whether the topic of speech is institutional or non-institutional (Pennington et al. 1992; Gibbons 1987; Lawson/Sachdev 2004). Through an analysis of language diaries as kept by adolescents in the first UK-born generation of the two focal groups of this study, we are able to gain a comparative view of the acculturation pattern of Bangladeshis and Chinese in the UK context that includes their use of the majority language, English, as well as maintenance of their 'heritage' languages of Bengali and Cantonese. 


\section{Background to the research}

\subsection{Bangladeshis and Chinese in the UK}

According to the UK Census (2001), the number of people in Great Britain who came from a minority (non-white) ethnic group grew by $53 \%$ in the last decade of the twentieth century, reaching 4.6 million in 2001. The number of people of non-European origin at the beginning of the twenty-first century comprised about $9 \%$ of the working-age population of England (Dustmann et al. 2003), over $38 \%$ of the population of inner-city London, and a much higher proportion in some neighborhoods (Office of National Statistics 2002). Over half of the non-European migrants in the UK population have come from South and East Asia (India, Pakistan, Bangladesh and Hong Kong). Census figures show Bangladeshis and Hong Kong origin Chinese making up $6.1 \%$ and 5.3\% respectively, of the non-white population of Britain (UK Census 2001).

Bangladeshis originally came to Britain as Bengali-lascars for the East India Company and later, in the 1960s, to work in the mills of West Yorkshire and Lancashire, and in engineering in the Midlands (Eade 1997). The majority of Bangladeshis in the UK today are of Muslim origin from the region of Sylhet, a rural part of northeastern Bangladesh (Eade 1990; Peach 2005). The language variety from Sylhet, referred to as Sylheti, is a vernacular variety that stands in a diglossic relationship with Bengali, the high-status, literate language of Bangladesh (Blackledge/Creese 2008; McPake/Sachdev 2008). Their common Bangladeshi background and their Muslim religious and social practices are important factors unifying Bangladeshis, as is the fact that they tend to concentrate in the same region of England, the southeast, especially London, and in London in the same neighborhoods of Camden and Tower Hamlets (UK Census 2001). According to Baker/Eversley (2000), Bengali and Sylheti constitute the second largest home languages (after English) of pupils in London's schools, accounting for $4.5 \%$ of the population. In five London school districts, Bengali and Sylheti rank first in terms of languages (other than English) being spoken in the home, reaching a high of over $50 \%$ of pupils in the Tower Hamlets district (McPake/Sachdev 2008).

The Chinese originally settled in the late nineteenth century in several port cities of the UK, opening businesses such as laundries, restaurants, and boarding houses aimed primarily at other Chinese customers. In the 1950s and early 1960s, ex-servicemen returning to Britain after their tours-of-duty in Pakistan, India, Singapore, Malaya, Hong Kong and Korea created a clientele for Asian cuisine and helped spread its popularity. Unlike the early immigrants, most of the Chinese migrants in the period since World War II were Cantonese-speaking with Hong Kong citizenship who came to Britain voluntarily to be employed in Chinese catering. Because of the direct flights and the number of Chinese restaurants there, London proved to be the most popular destination in Britain 
for Hong Kong migrants, although they did not concentrate in one area as their predecessors had (e. g., in East London). According to Owen (1994: 5), the distribution of ethnic Chinese in ten London boroughs ranged from 1.1 to $1.6 \%$ percent of the local population, with the highest percentage being found in Westminster which encompasses Soho and Chinatown. (For more detail on the history of the Hong Kong Chinese in London, see Lau 2003: Ch. 4).

Within the UK, Bangladeshis and Chinese represent contrasting groups on a number of features. An analysis of figures from the British Labour Force Survey for the years 1979-2000 shows that Bangladeshis, along with Pakistanis, had the highest level of unemployment and the lowest English language proficiency level of immigrant groups in the UK, whereas Chinese and Indians did well on both of these indicators (Dustmann et al. 2003; Modood 2005). A number of sources (e. g. Dustmann et al. 2003; Eade 1997; Modood 2005; Peach 2006; UK Census 2001) confirm that the UK Bangladeshis are a group suffering from a range of problems including: reduced employment opportunities and high unemployment, poor quality and overcrowded housing, poor health, low literacy and English language proficiency and low educational attainment. According to an annual Local Area Labour Force Survey (Office of National Statistics 2001/2002), both Bangladeshi and Chinese men (ages 16-64) had high male economic inactivity rates $-31 \%$ for each group - though the reasons for economic inactivity in these two groups were very different. Three-quarters of economically inactive Chinese men were students compared with just under half of inactive Bangladeshi men, $40 \%$ of whom were long-term sick or disabled. Bangladeshi women had the highest female economic inactivity rate at $78 \%$. The majority of these women were looking after their family or home. In terms of educational attainment, the 2001/2002 Labour Force Survey showed Bangladeshis as the least likely group to have degrees with approximately $44 \%$ having no qualifications, whereas fewer than $20 \%$ of Chinese were without qualifications. In addition, Chinese pupils were the most likely in England in 2002 to have high GCSE (General Certificate of Secondary Education) performance, achieving five or more GCSE grades A*-C (Bhattacharyya et al. 2003). Comparing the two groups, $77 \%$ of Chinese girls and $71 \%$ of Chinese boys had high GCSE performance whereas $50 \%$ of Bangladeshi girls and $40 \%$ of Bangladeshi boys had high GCSE performance.

Although relatively little sociolinguistic research exists on the two communities, there appear to be more studies on the London Chinese community (Lau 2001, 2003; Sachdev et al. 1991; Taylor 1987) than on the London Bangladeshi community (Lawson/Sachdev 2004). Recent research on Hong Kong born Chinese (Cantonese) adolescents in London (Lau 2001, 2003) suggests that members of this group who arrived in Britain at an early age and who socialize with friends from the majority group have a greater orientation to English and to the majority culture than those who do not. An orientation to the 
majority culture, as seen in their literacy practices and aspirations for the future, coexisted in this group of Cantonese adolescents with an orientation to the home community, as seen in practices involving Cantonese food, music, films, and social networks (Lau 2003). Like the Chinese adolescents observed by Hall/Sham (2007) in the Northwest of England, these London Chinese adolescents play an important bilingual role in functioning as 'language brokers' for their families even as they go beyond this mediator role to position themselves sociolinguistically in relation to the London speech community. As evidence of this positioning, Lau (2003) reports that the majority of the Chinese adolescents, and especially the females, adopt only middle class linguistic features (e. g., they have no double negatives).

Our research (Pennington et al. 2011) added similar comparative data for second-generation adolescent members of the London Cantonese and Bangladeshi communities. We found that while both groups are adopting features of the London vernacular, they did not show the same pattern of adoption of the forms and functions of London English. Both groups had a similar pattern of adoption of the 'urban-cool' discourse marker 'innit' and of non-adoption of the white-stereotyped glottal variant of $/ t /$, contrasted with distinctive usage of three other variables. The Bangladeshis had high occurrence of the non-standard feature of negative concord (double negative) coupled with low usage of high rising intonation in declaratives (HRT) and of be like used in quotative function, whereas the Chinese have low occurrence of negative concord, high occurrence of HRT, and moderate occurrence of be like in quotative function. This differing pattern of adoption of features of London English suggests that the Chinese are locating themselves through their linguistic performance more squarely in the middle class than are the Bangladeshis, who would appear to be positioning themselves by their linguistic usage more in alignment with the working class.

\subsection{Language and Identity}

Sociolinguistic and social psychological evidence shows that language choice is not merely concerned with communication efficiency or maintenance of the status quo. It is a central aspect of social and ethnic identity (Sachdev/Bourhis 2001, 2005; Giles et al. 1977; Sachdev/Giles 2004; Le Page/Tabouret-Keller 1985; Taylor/Moghaddam 1994), and it often aims to challenge the status quo (Blackledge/Creese 2008). In communities where the home language is different from that of the school and the larger society, the two languages will often be associated with different practical uses and symbolic values (Fishman 1980). Language serves as

a referent for loyalties and animosities, an indicator of social status and personal relationships, a marker of situations and topics as well as of the societal goals and the 
large-scale value-laden arenas of the interaction that typify every speech community (Fishman 1972: 4).

The mother tongue is generally associated in attitudes research with the values of tradition, community, solidarity, home, and family, whereas the language of the larger society is generally associated with the values of innovation or modernity, 'conspicuous consumption' and economic success, and academic achievement (Pennington 1994, 1995). By the second generation, an ethnic or minority group may "show a commitment to the enduring symbolic function of [the mother tongue] but not necessarily to its communicative function" (McNamara 1987: 226) [italics in original]. Thus,

they may show their affiliation to the mother tongue in terms of attitudes and affect but not in terms of actual usage. Thus, it may be that each succeeding generation of immigrants identifies with their ethnic language on an increasingly abstract level and less and less in terms of any concrete behaviors. There is always the chance in ethnic minority groups, however, of a social revival of mother tongue use, though without any definite societal function, the majority language will generally predominate. (Patri/Pennington 1998: 344)

Previous studies of Chinese and Indians in Hong Kong (Axler et. al. 1998; Patri/Pennington 1998; Pennington/Yue 1994; Pierson et al. 1980) have revealed a pattern of attitudes to English and their heritage languages that appears to represent "both an affirmation of their ethnic identity and a form of societal accommodation or communal identity" (Patri/Pennington 1998). In a study of Indians in Britain, Punetha et al. (1987: 230) found that these Asians "attempt to maintain their cultural values and traditions and to transmit them to succeeding generations." A recent study of Bangladeshis attending 'complementary' Bangladeshi schools in Birmingham, UK, found that these young people creatively used and evolved their own relationships to Bengali and Sylheti in ways that positioned them in opposition to traditional 'heritage' views of those languages:

The young people's attitudes to their languages, and their multilingual practices, constituted a sophisticated response to their place in the world, as they negotiated subject positions which took them on a path through language ideological worlds constructed by others. The young people were flexible and adaptable in response to their environment, as they negotiated identities which were more complex and sophisticated than the 'heritage' positions ascribed to them institutionally. (Blackledge/Creese 2008: 552)

\section{$3 \quad$ Methodology}

\subsection{Participants}

A total of 117 participants, 57 Bangladeshis (30 males, 25 females, and 2 of unknown gender) and 60 Chinese (16 males and 44 females) 13 to 18 year-olds 
randomly chosen from several London schools, kept a record of all language use for four days. Participants were recruited in areas of inner London (Camden, Kentishtown, Tower Hamlets, Westminster) - the Chinese from several Chinese (complementary) and mainstream schools and the Bangladeshis from mainstream schools and youth centers for Bangladeshis.

\subsection{Procedure}

Each student was provided with a bound diary booklet, which they kept with them for seven days after an orientation session in class. Following previous studies (Pennington et al. 1992; Pennington et al. 2005; Gibbons 1987; Lawson/Sachdev 2004), participants were instructed to record information about three main aspects of the interaction:

- context of conversation (time, place, circumstance, style, subject under discussion and who they were talking to);

- characteristics of others (interlocutors' education level, knowledge of languages, age, and ethnicity); and

- language medium (language or languages used in the interaction).

The booklets they were provided contained a sufficient number of pages formatted according to the categories of analysis. Participants were also asked to supply information about their background characteristics (e. g. sex, age, place of birth and language proficiency). For the purposes of the current research, the language choices were given as English, Bengali or Cantonese, and "mixed language'. Similar to the Lawson/Sachdev (2004) study, the differentiated mixed language categories reported were all collapsed into one category of 'mixed language' (either mixed Cantonese and English or mixed Bengali and English). Since this was a study with secondary school participants, the researchers determined that it would not have been possible to represent a more 'nuanced' classification of mixed language. The omission of the category of Sylheti was based on its very low reported occurrence in the Lawson/Sachdev (2004) study. $^{2}$

As a research method, language diaries are clearly open to the same criticism that can be leveled at any self-reporting methodology, i. e., that there can be

\footnotetext{
${ }^{2}$ It is impossible to know whether this low reporting of Sylheti was due to its actual low usage, participants' lack of clear differentiation of Sylheti/Bengali, the low status of Sylheti, and/or the high status of Bengali. Blackledge/Creese (2008: 544) noted that among the UK Bangladeshis in their study, those who spoke Sylheti, representing the majority of the Bangladeshis in Britain, thought that Sylheti and Bengali were "almost the same as each other".
} 
discrepancies between subjects' actual and reported behavior and that the task of completing a diary may itself influence participants' behavior in the phenomenon of 'reactance' (Breakwell 2006: 263). In addition, given the valueladen nature of language and its interconnections with personal and social identity, it is to be expected that a person filling out a diary that focuses attention on language may be led by underlying values and frames of reference to over- or under-report use of a certain language or variety. The language diary data is nevertheless a useful method for revealing participants' perceptions of their language choice. Language diaries supply information about participants' perceptions of the speech events they record, thus providing some insight into the motivations for their language behavior (Pennington et al. 1992; Gibbons 1987). Although completing a language diary requires a relatively high level of sustained co-operation on the part of the participants, it is nevertheless a type of task which has some elements of familiarity (e. g., from writing personal diaries and from filling out other types of forms) that make it a suitable task for younger participants.

\subsection{Diary data and analysis}

The following categories were derived from previous diary studies and from the patterns of our data:

\section{Setting}

- School: classroom; other school location.

- Private: speaker's home; home of relatives and friends.

- Ingroup public: youth centers; places of worship.

- Wider public: shops; parks; on public transport.

\section{Topic}

- School: school work; teachers; what happened in school.

- Social: what to do after school; gossip about other people.

- Family: members of the immediate family.

- Household: shopping; housework; meals.

- Media: what's on TV; music; films.

- Others: sports; etc. 
Interlocutor

- Older relatives: parents; aunts, uncles; etc.

- Younger relatives: siblings; cousins.

- Mixed relatives: with at least one each from the Older relatives and Younger relatives categories.

- Friends: participants' friends; classmates.

- Others: teachers; shopkeepers.

The categories of the data were derived for purposes of comparison with the previous diary studies. In the category of setting, 'School' was distinguished as a key setting in previous studies for use of English, whereas the 'Private' home setting was distinguished as a potentially key setting for use of heritage languages. Of public settings, a distinction was made between 'Ingroup public' settings such as youth centers and places of worship, where the heritage languages might be selected for communication with other speakers of those languages, versus 'Wider public' settings, those of the wider society where either English or heritage languages might be used depending on interlocutors and type of communication. Topics were categorized as those related to 'School' versus 'Social life' and 'home life', the latter divided into the two categories of 'Family' (people-focused) and 'Household' (not people-focused). Based on previous findings, 'Media' was distinguished as a topic where both English and heritage languages might be used. All other topics (e. g., sports) were placed in the category of 'Other'. Interlocutors were distinguished as those outside the family, both 'Friends' and non-peers or unknown or casually known 'Others' such as teachers and shopkeepers, and those inside the family, as this had proved an important dimension related to language choice in previous studies. Family members were categorized depending on whether they were 'Older relatives' (e. g. parents, grandparents, uncles, and aunts) or 'Younger relatives' (siblings and cousins) and thus closer to participants' age or not, or 'Mixed family' groups of older and younger relatives.

\subsection{Findings}

Each diary entry by each participant was considered as an individual data point. The diaries yielded a total of 1548 entries $($ Chinese $=622$, Bangladeshi $=926$ ) . Examination of overall patterns of participants' language use in Table 1 shows that the languages most widely spoken by participants were English for Chinese participants and Bengali for Bangladeshi participants. 
Table 1: Frequencies (and percentages) of diary entries about language use by ethnicity

\begin{tabular}{llcll}
\hline Group & $\begin{array}{c}\text { Total Number } \\
\text { of Entries }\end{array}$ & $\begin{array}{c}\text { Frequency and } \\
\% \text { Use } \\
\text { Heritage } \\
\text { Language }\end{array}$ & $\begin{array}{c}\text { Frequency and } \\
\% \text { Use English }\end{array}$ & $\begin{array}{c}\text { Frequency and } \\
\% \text { Mixed } \\
\text { Language Use }\end{array}$ \\
Chinese & 622 & $76,12.2 \%$ & $333,53.5 \%$ & $213,34.2 \%$ \\
Bangladeshi & 926 & $365,39.4 \%$ & $341,36.8 \%$ & $220,23.8 \%$ \\
& & $\chi^{2}=135, \mathrm{df}=2, \mathrm{p}<.001$ & \\
\hline
\end{tabular}

The Chinese participants reported using English most frequently $(53.5 \%)$ in their interactions whereas the Bangladeshi participants reported using Bengali most frequently (39.4\%), though their use of English (36.8\%) was reportedly nearly as high as that of Bengali. The Chinese group's use of their heritage language, Cantonese (12.2\%), was low relative to the Bangladeshi group's use of their heritage language, Bengali, though the Chinese adolescents reported a greater frequency of mixed language than the Bangladeshis (34.2\% vs. $23.8 \%)$. These overall patterns would appear to reflect the general dominance of English that is characteristic amongst second-generation immigrants in the UK. As is typical of multilingual communities (Sachdev/Bourhis 2001; Pennington 1994, 1998, forthcoming), substantial levels of mixed language were also reported by both groups.

Since an overall picture can mask important underlying patterns of variation, further analyses were conducted to see how multilingual behavior varied as a function of setting, topic, and interlocutor. Both groups of participants (B: Bangla; C: Cantonese) reported highest levels of English use in the institutional settings of school $(62.5 \% \mathrm{~B} ; 90.2 \% \mathrm{C})$ and those classified as in-group public settings $(75 \% \mathrm{~B}, 70 \% \mathrm{C})$, where the Bangladeshis (relative to the Chinese) reported substantial use as well of the heritage language in those contexts $(13.5 \% \mathrm{~B}, 4.2 \% \mathrm{C})$. In the wider public settings, reports of English use were high $(42 \% \mathrm{~B}, 39.6 \% \mathrm{C})$. Both groups reported substantial levels of mixed language $(30.7 \mathrm{~B} ; 46.8 \% \mathrm{C})$, with Chinese participants reporting a higher frequency of mixed language in the wider public settings than any other variety. In the private settings of the homes of family, relatives, and friends, although the use of heritage languages was stated to be high, it was Bangladeshi respondents who reported the highest use of their heritage language alone $(57.7 \% \mathrm{~B}, 17.5 \%$ C), while Chinese respondents reported more use of mixed English and Cantonese in the home setting $(51.9 \% \mathrm{C})$ than Cantonese alone.

For the Chinese participants, English reportedly dominated for all topics (over $50 \% \mathrm{C}$ for all) except those involving family members and household matters (under 30\%), where mixed language predominated (between 30-45\%) and where 
use of Cantonese was higher than for other topics (20-30\%). For Bangladeshi participants, Bengali predominated in the diary entries (over 40\%) except when discussing topics related to school (30.3\%) and social affairs (27.3), for which English was the dominant language choice (50\% or over).

For both groups, English was the most common choice recorded for speaking to those outside the family, including friends as well as non-intimate others (around $80 \%$ of the time for both groups). For the Chinese group, English was also recorded as the language used when speaking with younger relatives (53.7\%) whereas for the Bangladeshi group, Bengali was more often reported for speaking with this group (44.6\%). For speaking with older relatives, the Bangladeshis said they mainly used Bengali $(90.2 \%)$ whereas the Chinese said they mainly employed a combination of Cantonese and English (59.4\%). In family groups of mixed ages, the Chinese reported being even more likely to use the two languages together $(72.2 \%)$ whereas in mixed-age family groups the Bangladeshis reported using Bengali alone (50.8\%) and combinations of Bengali and English (46.8\%), with the reported use of unmixed Bengali predominating slightly in their reports of language use.

Thus, in contexts of intimacy defined by interlocutor and setting, the heritage languages and combinations of these with English were reported to be common, with the heritage language (Bengali) reportedly predominating for the Bangladeshi group, and mixtures of Cantonese and English predominating for the Chinese group. English was generally recorded as the language choice for both groups outside these contexts of intimacy. Within this general pattern, English was recorded to be the most common choice when speaking to those outside the family, and this included friends as well as others. Against the general pattern of the findings, the Chinese informants recorded English being used when speaking with younger relatives and mixed Cantonese and English occurring more frequently than English in wider public settings. The greater use of English with younger than older relatives, whether perceived or actual, may be a sign of increasing dominance of English down the generations within the London Chinese community as contrasted with the London Bangladeshi community.

In the context of Britain's civic ideology, private settings in the home and public in-group settings as operationalized in this study may be considered the most important bastions of in-group identity, and where the use of a heritage language (or languages) is valorized. Clearly, Bangladeshi participants' use of their heritage language in these settings, even if this is in part a matter of perception of the extent to which they report using it, augurs well for maintenance of their in-group identity and language, while the findings amongst Chinese participants suggest that they could be on their way to linguistic assimilation. Whereas a high level of use, or perceived use, of a heritage 
language in private settings would seem to be directly related to in-group identity, high levels (perceived or actual) of mixed language in those settings may signify both erosion and bilingual maintenance of the heritage language in the second generation. Language mixing has been reported to be especially common in the adolescent peer group, among the Cantonese-speaking youth in Hong Kong (Gibbons 1987; Pennington 1994, 1998, 2000) and more generally in other adolescent groups (Myers-Scotton 1993; Rampton 1995). The especially high frequency of participants' reports of mixed language in the Chinese group in the current study, as also reported for Cantonese-speaking people in Hong Kong and elsewhere - in the Northwest of England (Hall/Sham 2007) and in New Zealand ( $\mathrm{Ng} / \mathrm{He} 2004)$ - may signify that language mixing is a characteristic, and perhaps long-standing, feature of language use among Cantonese speakers which is not as strong a feature among Bangladeshis.

\section{Conclusion}

This language diary study has focused on the London-born children of two groups of migrants whose numbers in the UK, and specifically in London, have increased dramatically in the past 60 years, forming significant minority communities. In the case of the Bangladeshis, the migrant community is concentrated in a few areas whereas in the case of the Chinese, the community is more dispersed. As noted by Hall/Sham (2007: 19):

Families moving into a country using a principal language not spoken by them face many challenges. To some extent these can be mitigated either by moving into an area which has already been extensively settled by other members of their original community, or through being part of a network within which families can maintain ties with each other while geographically separated. In England, the first may be more typical of some South-Asian community groups while the second may be more typical of Chinese families. Either way there is a limit to the extent that any distinct minority language community can remain distanced from the social and institutional structures of the new national language group. Encounters with legal, medical, educational, administrative, social and economic aspects of life may well involve eventually, even if not initially, language interaction with people who do not speak or write the family language.

The results of the present study show significant adaptation to the majority English-speaking community by the first generation of UK-born children of Bangladeshi and Cantonese-heritage Chinese migrants to London. As compared to the Chinese, the Bangladeshi adolescents appear to be maintaining their heritage language to a greater extent. As compared to the Bangladeshis, the Chinese adolescents appear to use more mixed language. While London Cantonese-heritage Chinese adolescents may be assimilating linguistically at a faster rate than Bangladeshi adolescents to Anglo-British norms, they maintain a distinctive identity through mixed usage of English and Cantonese within their 
interactions with other Chinese. Whether this mixed-language feature of their communication and identity is transitional to monolingualism or will be maintained by speakers living in the Cantonese-heritage Chinese community in London, under conditions of continuing contact with Hong Kong and its people and culture, is a matter for further study.

The study reported here has provided rich data concerning self-reported language behavior as a function of the immediate sociolinguistic context. The findings show that the self-reported language behavior of the Chinese and Bangladeshi adolescents varied systematically as a function of setting, topic, and interlocutor, as these two groups of UK-born members of migrant communities negotiated their bilingual identities within the home and the surrounding context. The findings overall can be reported as these second-generation migrants adopting the main community language, English, while also maintaining the vitality of their heritage languages. In so doing, each of these groups adds its own distinctive contributions to the complex and ever-shifting patterning and interaction of languages and identities that has long been the profile of London.

\section{References}

Axler, Marie / Yang, Anson / Stevens, Trudy (1998). Current language attitudes of Hong Kong Chinese adolescents and young adults. In: Pennington, Martha C. (ed.): Language in Hong Kong at century's end. Hong Kong: Hong Kong University Press. 329-338.

Baker, Philip / Eversley, John (eds.) (2000). Multilingual capital: The languages of London's schoolchildren and their relevance to economic, social and educational policies. London: Battlebridge.

Bhattacharyya, Gargi / Blair, Maud / Ison, Liz (2003). Minority ethnic attainment and participation in education and training: The evidence. Research Topic Paper RTP01-03. Department for Education and Skills. Online: http://webarchive.nationalarchives.gov.uk/ 20130401151715/https://www.education.gov.uk/publications/standard/publicationDetail/P age1/RTP01 (latest retrieval [13.11.2013]).

Blackledge, Adrian / Creese, Angela (2008). Contesting 'language' as 'heritage': Negotiation of identities in late modernity. Applied Linguistics 29/4: 533-554.

Breakwell, Glynis M. (2006). Using self-recording: Diary and narrative methods. In: Breakwell, Glynis M. / Hammond, Sean / Fife-Schaw, Chris / Smith, Jonathan A. (eds.): Research methods in psychology. 3rd edition. London: Sage. 254-273.

Dustmann, Christian / Fabbri, Francsca / Preston, Ian / Wadsworth, Jonathan (2003). Labour market performance of immigrants in the UK labour market. Home Office Online Report 05/03. Online: /http://www.homeoffice.gov.uk/rds/pdfs2/rdsolr0503.pdf/ (last retrieval [06.06.2008]). 
Eade, John (1990). Nationalism and the quest for authenticity: The Bangladeshis in Tower Hamlets. New Community 16: 493-503.

Eade, John (1997). Keeping the options open: Bangladeshis in a global city. In: Kershen, Anne J. (ed.): London: The promised land? The migrant experience in a capitol city. Aldershot: Avebury. 91-105.

Fishman, Joshua A. (1972). The sociology of language: An interdisciplinary social science approach to language in society. Rowley, MA: Newbury House.

Fishman, Joshua A. (1980). Bilingualism and biculturalism as individual and as societal phenomena. Journal of Multilingual and Multicultural Development 1: 3-15.

García, Ofelia (2007). Foreword. In: Makoni, Sinfree / Pennycook, Alastair (eds.): Disinventing and reconstituting languages. Clevedon, UK: Multilingual Matters. Xi-Xv.

Gibbons, John (1987). Code-mixing and code choice: A Hong Kong case study. Clevedon: Multilingual Matters.

Giles, Howard / Bourhis, Richard Y. / Taylor, Don. M. (1977). Towards a theory of language in ethnic group relations. In: Giles, Howard (ed.): Language, ethnicity and intergroup relations. London: Academic Press. 307-348.

Hall, Nigel / Sham, Sylvia (2007). Language brokering as young people's work: Evidence from Chinese adolescents in England. Language and Education 21: 16-30.

Lau, Lawrence (2001). A foot in two worlds: A study of the language use and culture of British Hong Kong adolescents in London. Research Report 4/2. Language Research Centre, University of Bedfordshire.

Lau, Lawrence (2003). Cognitive and social factors explaining language acquisition: A study of the language of adolescents in the Hong Kong Cantonese community in London. Unpublished $\mathrm{PhD}$ thesis. University of Bedfordshire.

Lawson, Sarah / Sachdev, Itesh (2004). Identity, language use, and attitudes: Some SylhetiBangladeshi data from London, UK. Journal of Language and Social Psychology 23: 4969.

Le Page, Robert B. / Tabouret-Keller, Andre (1985). Acts of identity. Cambridge: Cambridge University Press.

May, Stephen (2005). Language rights: Moving the debate forward. Journal of Sociolinguistics 9/3: 319-347.

McNamara, Tim F. (1987). Language and social identity: Israelis abroad. Journal of Language and Social Psychology 6: 215-228.

McPake, Joanna / Sachdev, Itesh (2008). Community languages in higher education: Towards realising the potential. Southampton: University of Southampton LLAS.

Modood, Tariq (2005). Multicultural politics: Racism, ethnicity and Muslims in Britain. Edinburgh: Edinburgh University Press.

Myers-Scotton, Carol A. (1993). Social motivations for code-switching: Evidence from Africa. New York: Oxford University Press. 
Ng, Sik Hung / He, Anping (2004). Code-switching in trigenerational family conversations among Chinese immigrants in New Zealand. Journal of Language and Social Psychology 23: $28-48$.

Office of National Statistics (2002). Annual local area labour force survey 2001/02. Online: http://www.statistics.gov.uk/cci/ (latest retrieval [13.11.2013]).

Owen, David W. (1994). Chinese people and 'other' ethnic minorities in Great Britain: Social and economic circumstances. 1991 Census Statistical Paper 8. University of Warwick: Centre for Research in Ethnic Relations.

Patri, Mrudula / Pennington, Martha C. (1998). Minority and societal identity of Indian adolescents in Hong Kong. In: Pennington, Martha C. (ed.): Language in Hong Kong at century's end. Hong Kong: Hong Kong University Press. 339-362.

Peach, Ceri (2005). Britain's Muslim population: An overview. In: Tahir Abbas (ed.): Muslim Britain: Communities under pressure. London: Zed Books. 18-30.

Peach, Ceri (2006). South Asian migration and settlement in Great Britain, 1951-2001. Contemporary South Asia 15/2: 133-146.

Pennington, Martha C. (1994). Forces shaping a mixed code society: An interpretive review of the literature on language use and language attitudes in Hong Kong. Research Report 35. City University of Hong Kong: Department of English.

Pennington, Martha C. (1995). Pattern and variation in the use of two languages in the Hong Kong secondary English class. RELC Journal 26/2: 80-105.

Pennington, Martha C. (1998). Perspectives on language in Hong Kong at century's end. In Pennington, Martha C. (ed.): Language in Hong Kong at century's end. Hong Kong: Hong Kong University Press. 3-40.

Pennington, Martha C. (2000). Language innovation in Hong Kong. Research Report No. $3 / 2$. University of Luton: Language Research Centre.

Pennington, Martha C. (forthcoming). Introduction to bilingualism and multilingualism: People and languages in contact. New York: Wiley Blackwell.

Pennington, Martha C. / Balla, John / Detaramani, Champa / Poon, Anita / Tam, Frances (1992). Towards a model of language choice among Hong Kong tertiary students: A preliminary analysis. Research Report 18. City University of Hong Kong: Department of English.

Pennington, Martha C. / Lau, Lawrence / Chan, Brian Hok-shing (1998). Establishing discourse coherence bilingually: A comparison of code-switching and code-mixing modes. In: Caron, B. (ed.): Actes du 16e Congres International des Linguistes. Elsevier.

Pennington, Martha C. / Lau, Lawrence / Lee, Yuen-Ping (1996). Communicating in the Hong Kong secondary English classroom: The evolution of second language discourses. Research Monograph No. 7. City University of Hong Kong: Department of English.

Pennington, Martha C. / Lau, Lawrence / Sachdev, Itesh (2011). Diversity in adoption of linguistic features of London English by Chinese and Bangladeshi adolesecents. Language Learning Journal 39/2: 177-199.

Pennington, Martha C. I Sachdev, Itesh (2013). Language attitudes and ethnolinguistic identity of British-born Bangladeshis and Chinese. Unpublished ms. 
Pennington, Martha C. / Yue, Francis (1994). English and Chinese in Hong Kong: Pre-1997 language attitudes. World Englishes 13: 1-20.

Pennington, Martha C. / Yue, Francis / Detaramani, Champa / Young, Jean (2005). Language choice among Hong Kong tertiary students in the new millennium. Research Report. City University of Hong Kong.

Pierson, Herbert D. / Fu, Gail S. / Lee, Sik-yum (1980). An analysis of the relationship between the language attitudes and English attainment of secondary school students in Hong Kong. Language Learning 30: 289-316.

Punetha, Deepa / Giles, Howard / Young, Louis (1987). Ethnicity and immigrant values. Journal of Language and Social Psychology 6: 229-241.

Rampton, Ben (1995). Crossing: Language and ethnicity among adolescents. London: Longman.

Sachdev, Itesh / Bourhis, Richard Y. (1991). Power and status differentials in minority and majority group relations. European Journal of Social Psychology 21: 1-24.

Sachdev, Itesh / Bourhis, Richard Y. (2001). Multilingual communication. In: Robinson, Peter / Giles, Howard (eds.): The new handbook of language and social psychology. Chichester and New York: Wiley and Sons. 407-428.

Sachdev, Itesh / Bourhis, Richard Y. (2005). Multilingual communication and social identification. In: Harwood, Jake / Giles, Howard (eds.): Intergroup communication: Multiple perspectives. New York: Lang. 65-92.

Sachdev, Itesh / Bourhis, Richard Y. / Phang, Sue-wen / D’Eye, John (1987). Language attitudes and vitality perceptions: Intergenerational effects amongst Chinese Canadian communities. Journal of Language and Social Psychology 6: 287-307.

Sachdev, Itesh / Giles, Howard (2004). Bilingual accommodation. In: Bhatia, Tej K. / Ritchie, William C. (eds.): Handbook of bilingualism. Oxford: Blackwell. 353-378.

Sachdev, Itesh / Giles, Howard / Pauwels, Anne (2012). Accommodating multilinguality. In: Tej K. Bhatia / William C. Ritchie (eds.): Handbook of bilingualism and multilingualism. Oxford: Blackwell. 391-416.

Tarone, Elaine (1988). Variation in interlanguage. London: Edward Arnold.

Taylor, Donald M. / Moghaddam, Fatthali M. (1994). Theories of intergroup relations: International social psychological perspectives. Westport, CT, and London: Praeger.

Taylor, Monica J. (1987). Chinese pupils in Britain: A review of research into the education of pupils of Chinese origin. Windsor: NFER-Nelson Publishing Co.

U.K. Census (2001). National Statistics website. Online: www.statistics.gov.uk/census2001 (latest retrieval [13.11.2013]) 



\title{
Le développement plurilingue et interculturel en milieu éducatif ouvert à la diversité - étude et bilan de trois projets universitaires avec la participation d'une écologie linguistique « à la luxembourgeoise »
}

\author{
Sabine Ehrhart (Luxembourg)
}

\begin{abstract}
Die aktuelle Forschung trennt nicht mehr kategorisch zwischen natürlichem Spracherwerb und institutionellem Sprachenlernen. Gerade durch das Miteinbeziehen von kulturellen Aspekten in möglichst authentischen Lernumgebungen entstehen ganz neue Situationen für die Lernenden in mehrsprachigen Umgebungen. Die langjährige Mitarbeit in LANGSCAPE und der dort erfolgte Austausch mit Vertretern von mehrsprachigen Universitäten haben mich auch bei eigentlich von LANGSCAPE unabhängigen Projekten inspiriert, nämlich bei LACETS (2008-2011), UniGrR (2009-2012) und TEMPUS IV (2010-2013).

Современное исследование больше не разделяет так категорично естественное усвоение и целенаправленное изучение языка. Совершенно новые возможности для учащихся в многоязычных средах возникают вследствие привлечения культурных аспектов в аутентичную учебную среду. Многолетняя работа в Langscape и имевший место обмен опытом с представителями многоязычных университетов сподвигли меня к собственно независимым от Langscape проектам, таким как LACETS (2008-2011), UniGrR (2009-2012) и TEMPUS IV (2010-2013).
\end{abstract}

\section{Introduction}

Traditionnellement, le succès dans l'apprentissage d'une langue était mesuré par la maîtrise des structures de celle-là. Le présent article propose une approche plus globale et postule que dans l'établissement de contact avec une nouvelle langue, le contexte social, la motivation d'apprendre et d'autres facteurs socioet psycholinguistiques jouent un rôle primordial (cf. Ledegen 2007; Perrefort 2008) au même point que les éléments linguistiques rencontrés. En tant qu'acteur dans plusieurs projets scientifiques dans le domaine de l'éducation, j'ai pu expérimenter l'importance de l'ouverture à la diversité linguistique et culturelle à la fois comme sujet d'étude ou bien en tant que participante à un projet (de Mejía et al. 2006). Les rencontres organisées par LANGSCAPE m'ont aidée à approfondir ces sujets avec des partenaires venant d'horizons très divers. Ces échanges ont enrichi mon travail dans les projets précis avec des partenaires n'appartenant pas au groupement LANGSCAPE et c'est pour cette raison que j'ai décidé de les présenter dans cet ouvrage qui dresse un bilan des activités menées dans ce mouvement. J'ai délibérément choisi la forme d'un Werkstattbericht ('rapport d'atelier') pour ce texte car il me semble que de tels bilans ne sont pas suffisamment divulgués, alors qu'ils pourraient être utiles pour un plus grand 
public, notamment pour ceux qui planifient des colloques ou des collaborations de visée internationale.

Dans le cadre de la classification établie par LANGSCAPE, ma contribution s'inscrit dans le domaine des contextes de mobilité et de l'écologie des langues (Calvet 1999; Creese/Martin 2003; Ehrhart 2012a). Mes descriptions se réfèrent notamment aux défis rencontrés lorsque l'on travaille avec une pluralité de langues dans des contextes internationaux ou globaux (García 2009; Lüdi 1996). Dans les contextes que j'ai étudiés il n'est pas toujours aisé de distinguer entre langue maternelle, langue étrangère ou langue seconde et $\mathrm{j}$ 'utiliserai ces termes avec beaucoup de précaution en donnant une description plus détaillée de chaque situation quand le besoin s'en fera sentir. Par ailleurs, dans mon cas, l'aire géographique couverte peut parfois dépasser le contexte européen.

Dans ma présentation de trois exemples de projets de développement plurilingue et interculturel en milieu éducatif ouvert à la diversité, je ne traiterai pas les trois exemples de la même manière. Après une brève comparaison des paramètres qui ont défini les trois projets, je mettrai l'accent sur le projet LACETS que j'ai pu créer et diriger pendant six ans et sur les contenus desquels j'ai eu un impact plus direct que sur ceux des deux autres. Le projet de recherche LACETS, le séminaire UniGR et le groupe linguistique de TEMPUS sont unis par le souci de développer des orientations innovantes pour la formation des enseignants ou formateurs dans le domaine de la pluralité et de l'ouverture à l'autre, cette approche ne se limitant pas aux enseignants de langues.

\section{Comparaison du cadre}

Dans ce qui suit sera donnée une brève description des trois projets étudiés à travers les paramètres qui ont défini leur mise en place (espace, temps, rythmes des rencontres, types de participants, ancrage disciplinaire, liens sociaux et caractéristiques des langues).

\subsection{Enracinement géographique}

LACETS (Langues en contact dans l'espace et dans le temps et leur impact sur le milieu scolaire au Luxembourg): ce premier projet était situé au Luxembourg en tant que projet de recherche interne à l'université du Luxembourg; je l'ai créé peu de temps après mon arrivée au Luxembourg en 2006 afin de travailler sur la sensibilisation à la diversité linguistique et culturelle dans la formation des futurs enseignants du primaire et du secondaire dans le pays. Ce projet de recherche appliquée a eu une première phase de démarrage entre 2006 et 2009 et ensuite un travail plus vaste avec une équipe de deux ou trois personnes pendant la deuxième phase située entre 2009 et 2011. C'est de cette phase que je vais 
parler ici car elle comporte une plus grande orientation internationale, aussi bien au sein des acteurs de recherche que dans les sujets étudiés. En outre, elle est plus directement comparable à la durée des deux autres projets. Ce projet trouve une prolongation avec NaturaLink, un projet qui étudie davantage les approches naturelles dans l'appropriation des langues et que je mène actuellement avec Sarah Vasco, à l'université du Luxembourg également.

UniGrR: ce projet s'est déroulé dans le cadre de l'université de la Grande Région. Avec des collègues de l'Universität des Saarlandes à Saarbrücken en Allemagne et de l'Université de Lorraine à Metz en France, nous avons créé un séminaire trinational conjoint sur la gestion du plurilinguisme avec notamment, de futurs enseignants et chercheurs de nos trois universités. L'ensemble du groupe, professeurs et étudiants confondus, s'est déplacé sur les trois sites universitaires et a expérimenté l'ambiance didactique de chaque lieu de manière collective. J'ai guidé la délégation luxembourgeoise dans ce projet et nous avons organisé un séminaire trinational par an en 2009, 2010 et 2011. L'université de la Grande Région a fonctionné comme projet de manière virtuelle jusqu'en avril 2013, elle prendra une forme plus durable à partir de cette date.

TEMPUS (TEMPUS IV 159328-TEMPUS-FR-TEMPUS-SMHES Life-LongLearning Framework for Medical University Teaching Staff): ce dernier projet est de grande envergure et il a relié plusieurs partenaires européens à un ensemble d'universités russes, avec l'objectif d'instruire des formateurs dans les académies de médecine de ce vaste pays. TEMPUS est financé par la Commission Européenne et a comme but de moderniser l'enseignement supérieur dans les pays voisins de l'Union Européenne. Un projet précurseur a fonctionné avec une équipe plus petite (Omsk-Strasbourg-Luxembourg) avec une forte présence de la langue française avant de passer principalement à l'anglais pendant la phase d'agrandissement. J'ai dirigé le groupe de recherche linguistique et interculturelle dans ce projet, en étroite collaboration avec mes partenaires russes. Une demande de prolongation dans le cadre de TEMPUS V et TEMPUS VI n'a pas été retenue, mais l'équipe continuera sa coopération sous d'autres formes plus libres, comme un travail sur la motivation d'apprendre des langues par les étudiants des universités partenaires.

\subsection{La durée des projets}

La durée a été de trois ans pour chacun des projets. La durée avait été prévue pour LACETS et TEMPUS, quant à UniGR, nous avons prolongé un contrat sur une année, à deux reprises, sans savoir si la demande de prolongation allait aboutir.

En principe, le cadre plus sûr d'un contrat fixé sur trois ans permet davantage de planifier dans le temps et de construire des systèmes de collaboration 
durables, mais l'absence de contrat pour le deuxième projet a été récompensée par la souplesse et la flexibilité de ses acteurs.

Comme je l'ai expliqué dans le chapitre précédent, les trois projets s'inscrivent dans une perspective plus vaste et dans une durée plus longue. Les trois projets ont été suffisamment motivants pour que nous ayons envie de continuer avec nos partenaires et pour chacun des trois, notre collaboration trouve actuellement une continuation sous d'autres conditions (publications conjointes, organisation de colloques et de journées d'études en synergie, fonction d'experts dans des processus d'évaluation etc.).

\subsection{Le lien entre temps et espace: le type de mobilité engagée}

En fonction de l'espace couvert, le type de mobilité varie considérablement d'un projet à l'autre. Alors que les membres de LACETS se trouvaient dans le même bureau, sur le même campus, dans la même ville et le même pays tout en ayant des nationalités bien différentes (allemande, française, australienne et luxembourgeoise), les deux autres projets demandaient à chaque rencontre de franchir des frontières géographiques et de faire des trajets plus ou moins longs. Grâce aux moyens de communications actuelles, la distance n'a pas toujours été ressentie comme un obstacle, le facteur 'éloignement' donne parfois un attrait exotique qui peut être porteur ou au moins accompagnateur d'un échange scientifique et humain. Toutefois, des rencontres personnelles et régulières sont nécessaires si la recherche veut porter ses fruits; de simples échanges virtuels peuvent faire passer de l'information claire et précise, mais sans s'immerger dans le contexte matériel et culturel du partenaire, un chercheur ne peut pas pleinement développer l'esprit de communauté qui est à la base d'une création commune de qualité.

\subsection{Le profil des participants, les objectifs et le public visé}

LACETS a choisi l'école maternelle et primaire au Luxembourg comme terrain de recherche, les collaborateurs scientifiques étaient des doctorants ou avaient un niveau post-doc, ils étaient étudiants mais aussi professeurs d'université. Les autres membres et collaborateurs étaient des enseignants diplômés allant du diplôme d'Etat luxembourgeois au doctorat. Le groupe cible avec lequel nous voulions surtout partager les résultats de nos recherches étaient les étudiants en formation pour être enseignants à l'école maternelle et primaire au Luxembourg. Nous avions l'espoir que ces résultats puissent aussi apporter des éléments intéressants pour la gestion de la diversité linguistique et culturelle dans les écoles d'autres pays. 
Dans le séminaire UniGR, les professeurs des trois pays sont restés les mêmes, avec quelques petits changements dus à la mutation de la collègue sarroise en allemand langue étrangère. D'une année à l'autre, les étudiants ont changé, la continuité était assurée par les professeurs et leurs équipes sur place dans chacun des trois pays. Le thème central dans ce projet était la gestion des langues dans l'enseignement supérieur et surtout secondaire.

TEMPUS s'est créé autour de deux personnes phares, un médecin russe francophone et un médecin franco-luxembourgeois russophone, deux éminents spécialistes en pneumo-oncologie qui ont créé une équipe autour de ce partenariat motivant et porteur. L'orientation de TEMPUS, une initiative fondée par la Commission Européenne, est d'apporter l'innovation dans les pratiques disciplinaires de l'enseignement supérieur des pays voisins de l'Union Européenne. C'est la deuxième phase du projet entre 2009 et 2012 qui avait davantage mis l'accent sur la formation des formateurs, médecins et professeurs de langues dans les Académies de Médecine des villes partenaires russes. La première tranche du projet avait montré l'importance d'une approche interculturelle en parallèle avec la formation purement médicale, notamment pour préparer et accompagner les stages à l'étranger qui pouvaient avoir une durée de plusieurs mois, voire des années. Une formation disciplinaire est plus prometteuse si elle se fait dans de bonnes conditions sociales et humaines, le processus d'apprentissage et de mémorisation peut être freiné ou même bloqué par un trop-plein de nostalgie et de mal-être dans le pays étranger (Perrefort 2008). C'est dans ce contexte que le groupe linguistique et interculturel s'est constitué. Bien qu'intéressés particulièrement par la langue de discipline, le français pour les médecins et les métiers paramédicaux, notre groupe linguistique et interculturel avait pris soin de ne pas adopter une attitude ancillaire par rapport à la médecine, mais de nous affirmer comme discipline indépendante et partenaire égale de celle-ci.

\subsection{Les langues utilisées}

Les langues utilisées n'ont pas été les mêmes tout au long des projets. Les lieux de rencontre et surtout les compétences linguistiques des participants ainsi que leur potentiel de développement ont été les facteurs les plus déterminants.

LACETS avait commencé avec deux Allemandes, la directrice du projet trilingue dans les langues officielles de l'Université (français-allemand-anglais, la dernière étant la plus faible dans le trio) et une bilingue (allemand-portugais), les principales langues d'observation pour le groupe d'élèves choisi. Elles avaient été rejointes par un Australien ayant des origines françaises qui parlait l'anglais et le français, la première étant la langue la plus forte. Les zones de recoupage linguistique ont été quasiment nulles entre la deuxième Allemande et 
le nouveau collaborateur. Entre la directrice et le collaborateur, le français a été la langue la plus fréquemment utilisée et l'anglais était utilisé dans les conversations sous forme de bilinguisme réceptif (une personne parlait en français, une en anglais, chacune dans sa langue la plus forte), pour certaines tâches et à des moments précis du projet. Au milieu du projet, la première collaboratrice a dû abandonner le projet pour des raisons familiales et une nouvelle collaboratrice a été engagée pour le temps restant comme secrétaire scientifique. Comme elle parlait et écrivait les trois langues universitaires, le lien entre les membres s'est renforcé et des échanges plus profonds se sont développés, notamment de manière directe entre les collaborateurs, sans qu'ils aient toujours recours à la directrice du projet.

TEMPUS était au départ un projet franco-russe dans sa première phase et au tout début, l'emploi de l'anglais avait été vivement critiqué à certains moments (notamment quand par manque de traducteurs qualifiés franco-russes, on avait fait appel à des traducteurs anglais-russe). Pour la deuxième phase entre 2009 et 2012, l'anglais a tout de suite pris une place centrale, car le projet s'était ouvert à une dizaine d'autres universités russes dont la plupart des membres mâ̂trisaient davantage l'anglais que le français. Les coordinateurs du projet basés à Omsk étaient généralement plus à l'aise en anglais, avec des connaissances partielles pour certains et une maîtrise bilingue pratiquement équilibrée pour d'autres. La branche luxembourgeoise du projet a regretté de ne pas parler le russe, mais le temps n'a pas suffi pour combler cette lacune. Mis à part le noyau dur du projet et les directeurs de programme, les membres présents aux différentes réunions n'étaient pas toujours les mêmes, ce qui n'a pas permis d'améliorer concrètement les compétences dans la langue de l'autre.

Dans le projet du séminaire trinational, trois groupes se sont rencontrés, en provenance de la France, du Luxembourg et de l'Allemagne. En ce qui concerne le professorat, il y avait un bilinguisme franco-allemand (ou mosellan) plus ou moins développé et la communication se déroulait aisément dans une langue commune, même pour les sujets complexes. Parmi les étudiants (une quinzaine de Sarrois, et une demi-douzaine en provenance de la Lorraine et du Luxembourg), la situation était différente. Les Sarrois avaient en règle générale de bonnes voire de très bonnes connaissances en langue française. Pour des sujets très techniques ou complexes, les francophones devaient parfois légèrement réduire le débit de leurs paroles pour être parfaitement compris. Les Lorrains étaient d'origine très diverse ayant une ouverture interculturelle très riche, mais parmi les langues qu'ils maîtrisaient, seulement le français avait un dénominateur commun avec les étudiants des autres pays. Les autres langues parlées (du Caucase, des Balkans, du Maghreb...) ne pouvaient pas être employées comme moyen d'échange général. Les Luxembourgeois étaient au moins plurilingues en luxembourgeois, français, allemand et anglais, parfois, ils avaient encore d'autres langues à leur disposition comme l'italien, l'espagnol ou le portugais. 
Après un moment d'hésitation où ils se comparaient aux étudiants des deux autres pays se caractérisant par des habitus davantage monolingues, ils ont osé parler dans plusieurs de leurs langues malgré la représentation parfois assez négative qu'ils se faisaient de leurs compétences linguistiques notamment dans les langues principales des pays voisins, la France et l'Allemagne. D'après eux, leur maîtrise n'était pas à 100\% parfaite de chacune des langues utilisées, le français et l'allemand et cela leur causait une gêne pas toujours compréhensible pour les étudiants originaires des autres pays, surtout comme dans la majorité des cas, elle n'était pas accompagnée d'un manque structural visible ou audible. Du point de vue de leurs collègues venant de France et d'Allemagne, ils semblaient être complètement à l'aise en allemand, en français et souvent aussi en anglais et le passage entre les langues se faisait avec une grande facilité comparée aux participants ayant suivi une scolarité dans un autre pays à tendance plutôt monolingue.

D’une manière générale, la langue principale pour les échanges pour tous les participants fut donc le français, avec quelques éléments en allemand qui étaient fréquemment traduits pour assurer la compréhension complète des francophones.

\subsection{Les stratégies de communication plurilingues employées}

Une question centrale qui se pose quand on regarde les projets d'un point de vue linguistique, notamment écolinguistique, est celle de la circulation du savoir: quels formes et modèles linguistiques et culturels ont été mis en pratique afin d'assurer le bon fonctionnement des échanges (Byram/Dervin 2008)? En outre, il serait intéressant de savoir si la durée des projets a été suffisamment longue pour faire évoluer les habitudes linguistiques des partenaires, entre le début et la fin des projets.

Un trait commun entre LACETS, TEMPUS et UniGR est le fait que le sujet d'étude et la manière pratique de travailler ont été fortement liés dans le déroulement de la recherche. Les trois projets ont eu comme sujet d'étude la circulation des savoirs et la communication en milieu éducatif plurilingue et multiculturel, mais ils ont eu des manières différentes de mettre en pratique la gestion les ressources disponibles. Ce qui les unit encore une fois est le fait que pour aucun des projets, une politique linguistique explicite ait été mise en place avant ou pendant la durée des échanges. LACETS a essayé de réaliser les échanges à l'intérieur de l'équipe - et aussi au-delà - de manière flexible, UniGR a utilisé la traduction et l'interprétariat ad-hoc avec les membres luxembourgeois fortement plurilingues comme pivots et TEMPUS a développé notamment la capacité de décoder les niveaux para-verbal et non-verbal, car les langues employées (français ou anglais et russe) n'étaient nullement intercompréhensibles et rarement 
maîtrisées toutes les deux par les mêmes personnes. En règle générale, trois ans de recherche commune n'ont pas suffi pour développer ou rapprocher les compétences linguistiques des différents membres. Cependant, tout au long de ces années, nous avons observé un changement dans les attitudes des participants vers la compréhension interculturelle et la volonté plus forte de collaboration, et ceci dans le rythme de travail des trois projets.

\subsection{Fruits et rendements}

Dans les trois projets que nous décrivons, on peut observer un rendement scientifique riche, malgré les barrières linguistiques et/ou culturelles: LACETS a organisé des colloques, publié un livre et plusieurs articles et les résultats obtenus continuent à contribuer à la formation des enseignants au Luxembourg et dans d'autres pays (Madagascar, Nouvelle-Calédonie, Roumanie). UniGR a créé une base de collaborations scientifiques entre plusieurs pays (colloques, séminaires, publications d'articles et de documents numériques, comme un grand séminaire avec l'Université Ouverte des Humanités avec la publication de matériel en ligne; cf. Trepos et al. 2013). TEMPUS a donné lieu à plusieurs productions écrites comme résultat de notre collaboration (Ehrhart 2012; Markovina et al. 2013; Musochranowa/Ehrhart 2008). Ces publications se situent soit en médecine générale dans différentes disciplines, soit en linguistique appliquée et en didactique des langues avec notamment un manuel d'accompagnement interculturel pour les médecins russes qui se rendent dans d'autres pays pour raisons professionnelles.

\section{Présentation détaillée d'un projet portant sur la construc- tion d'identité et l'éducation des langues}

\subsection{Localisation géographique et sociolinguistique du projet}

Dans ce qui suit, j'ai choisi de présenter plus en détail les objectifs, le déroulement et une partie des résultats d'analyses du projet LACETS - Langues en contact dans l'espace et dans le temps et leur impact sur le milieu scolaire au Luxembourg. Des trois projets présentés auparavant, c'est celui sur lequel j'ai pu avoir le plus d'impact direct et j'ai essayé d'y mettre en œuvre une attitude d'écologie linguistique respectueuse de l'altérité, aussi bien dans le choix des sujets d'étude que dans l'organisation de la recherche conjointe.

Ce chapitre portera donc sur les résultats partiels du projet Lacets qui a été mené à l'Université du Luxembourg de septembre 2008 à septembre 2011 sous ma direction et qui comprenait des collaborateurs ayant des profils divers, aussi bien en provenance des écoles que de l'université. En septembre 2011, à la fin du projet, nous avons présenté un rapport final destiné à l'administration de 
notre université. Nous reprenons ici les éléments de ce rapport interne qui pourraient intéresser un plus grand public.

Les lacets dans le titre du projet symbolisent le développement du lien social dans la vie d'un être humain. D'après les enseignantes, le fait de savoir nouer ses lacets est un indicateur de maturité qui peut également être relié à la capacité de socialisation. C'est le premier stade de la vie que nous avons observé dans notre projet. A l'approche de l'adolescence, le jeune montre l'envie de ne plus nouer les lacets de ses chaussures et il le vit comme un refus des normes de la société qui l'entoure. Cette attitude correspond au deuxième groupe d'âge qui nous a intéressés pour le projet LACETS, celui des élèves de l'enseignement secondaire. Principalement, nous avions centré notre intérêt sur l'école maternelle et l'école primaire qui sont regroupées sous le toit de l'école fondamentale depuis la réforme scolaire de 2009/2010. Par la suite, nous avons élargi notre horizon de recherche aux écoles secondaires, afin de pouvoir suivre l'ensemble de la biographie de l'apprenant scolaire.

Nous nous étions fixé pour objectif d'échanger les résultats de notre recherche de manière intensive, puis de le mettre en pratique à travers de nombreuses activités au niveau national et international. Nous avons organisé plusieurs journées d'études, des ateliers de recherche, des symposiums et des conférences internationales au Luxembourg. Lors de conférences à l'étranger, nous avons présenté les résultats de notre recherche, ainsi que le modèle luxembourgeois de la gestion des langues à l'école et nous avons réalisé une série de publications dans des revues de renom ainsi qu'un livre sur la formation des enseignants en milieu plurilingue (Ehrhart e al. 2010). Notre objectif était de montrer des exemples d'enseignement dans des environnements caractérisés par une pluralité des langues et cultures qui seraient susceptibles de servir d'exemples de bonne pratique, d'encouragement et de source d'inspiration au-delà des frontières de leur pays d'origine.

Sur la base des travaux de LACETS, j'ai pu développer des modules d'enseignement pour différents secteurs de formation des enseignants au Luxembourg, parmi lesquels je citerai en premier lieu le Cours 2 - Plurilinguisme, cultures et langues à l'école pour la formation des enseignants du secondaire. Ce séminaire qui est obligatoire pour l'ensemble des stagiaires traite les sujets du plurilinguisme individuel et du multilinguisme collectif, de la didactique du plurilinguisme, de l'approche interculturelle et de la mise en valeur ethnolinguistique de la diversité linguistique de la classe et il se base directement sur les résultats de la recherche du projet LACETS.

Pendant la durée du projet, les membres de LACETS donnaient en moyenne 200 heures d'enseignement universitaire annuel à l'Université du Luxembourg dans des domaines qui se rapprochent des objectifs fixés auparavant comme la didactique du plurilinguisme, la gestion de la diversité linguistique et culturelle 
en classe et l'écologie linguistique. Ainsi, entre 2008 et 2011, j'ai régulièrement enseigné les cours Conceptualiser et réaliser des séries d'enseignement et d'apprentissage et Développer des approches interdisciplinaires à l'apprentissage au sein de la Formation Pédagogique pour les Enseignants du Secondaire au Luxembourg, un module L'écologie de la classe pour le BScE (Bachelor en Sciences de l'Education). J'ai également organisé des séminaires en formation continue pour le SCRIPT, organisme de formation du Ministère de l'Education au Luxembourg, avec des cours intitulés: Kannersproochen (Les langues des enfants; avec Evelyne Roth-Dury); Eveil aux langues et Gérer la complexité des situations d'apprentissage ainsi qu'un module d'accueil pour les étudiants ERASMUS à la Faculté des Lettres de l'Université du Luxembourg intitulé $\mathrm{La}$ mobilité et l'ouverture aux langues et aux cultures en février et mars 2010. Au sein de la formation pédagogique, je suis coordinatrice de module et membre du comité de pilotage de la formation pédagogique et représentante du corps des enseignants-chercheurs de la Faculté des Lettres au conseil universitaire ce qui me permet de rappeler l'importance d'une gestion raisonnée du plurilinguisme à différents niveaux de la structure universitaire.

Actuellement, en 2013, des résultats de LACETS inspirent toujours les enseignements au sein de notre université: le cours sur le plurilinguisme est installé de manière durable dans le programme de la formation pédagogique, j'accompagne des travaux de bachelor des futurs professeurs du primaire portant sur l'écologie linguistique de la classe et j'enseigne dans un Master dédié au plurilinguisme, également au sein de notre université. Plusieurs projets internationaux (dont les deux présentés au chapitre 2 de ma contribution) profitent des expériences de LACETS pour aller plus loin dans la communication interculturelle dans le domaine éducatif et scientifique.

Le projet de recherche LACETS 2 était domicilié à la Faculté des Lettres, des Sciences Humaines, des Arts et des Sciences de l'Education de l'Université du Luxembourg (FLSHASE), aujourd'hui Faculté des Lettres, et il était étroitement lié à la priorité de recherche "L'éducation et l'apprentissage en contexte multilingue et pluriculturel » qui a fait partie du programme du plan quadriennal universitaire de 2010 à 2013. Ses activités s'inséraient dans celles de l'unité de recherche LCMI (Languages, Culture, Media and Identities) et plus particulièrement de son aire de recherche LEPL (Language Ecology and Plurilingual Literacies, aujourd'hui Institut pour le Plurilinguisme). Son domaine de recherche est le multiplurilinguisme scolaire au Luxembourg. Du point de vue des disciplines traditionnelles, LACETS se situe au point de rencontre entre les domaines de la sociolinguistique, de la linguistique appliquée et celui de la politique linguistique. Il observe (par une approche ethnométhodologique) et il analyse (en utilisant, entre autre, l'analyse critique du discours) des attitudes et des stratégies de communication employées à l'école plurilingue, entre les enseignants et les élèves et entre les élèves eux-mêmes, dans des situations où 
différentes langues sont présentes et/ou employées. L'écologie linguistique de la classe propose de créer un lien dynamique entre les sphères individuelles et collectives, notamment entre les ressources d'un élève et la richesse de la classe considérées comme un ensemble. C'est pour cette raison que nous avons choisi cette nouvelle orientation scientifique comme ligne directrice. Au cours des trois années de durée du projet de septembre 2008 à septembre 2011, nous avons tenté de développer un nouveau cadre conceptuel holistique, dynamique et pluridimensionnel pour l'interprétation de la diversité linguistique et culturelle des élèves luxembourgeois ayant pour objectif la création de nouvelles pistes pour l'aménagement de l'espace éducatif plurilingue. Nous avons particulièrement observé les endroits où plusieurs langues étaient en contact, notamment quand ce contact a été provoqué par la mobilité ou la migration d'une partie de la population observée. La mention 'et dans le temps' dans le titre indique que notre démarche était celle d'une étude longitudinale qui a cherché dans le présent et le passé des situations plurilingues les clés pour une organisation harmonieuse de l'avenir de la société.

\subsection{LACETS dans le contexte national luxembourgeois}

Cette partie se limite à décrire les moments décisifs dans le déroulement et le rayonnement du projet, dans une démarche de recherche-action en citant des activités multiples adressées à un public très divers: élèves, parents d'élèves, enseignants de l'école fondamentale, de l'enseignement secondaire et universitaire, chercheurs, formateurs, responsables administratifs et décideurs politiques.

Le Luxembourg compte parmi les pays européens les plus riches en langues: cette diversité linguistique s'explique par sa situation historiquement fondée à la limite des mondes germanophone et " romanophone " (Ehrhart/Fehlen 2011c: 15), et par une mobilité démographique actuelle très forte (Ehrhart 2012b). On observe notamment une importante immigration au cours du 20ème siècle qui a amené des locuteurs de nombreuses langues européennes et non-européennes dans ce pays d'un demi-million d'habitants et d'une superficie de $2586 \mathrm{~km}^{2}$. Il est intéressant de constater que les différentes langues qui sont parlées au Luxembourg ne sont pas réparties par zones géographiques (dans un multilinguisme selon le modèle suisse) ou isolées dans des compétences individuelles (dans le plurilinguisme typique de nos grandes métropoles par exemple), mais qu'elles se côtoient et s'entrecroisent de manière intense dans les interactions sociales de chaque personne; les individus monolingues sont pratiquement inexistants au Luxembourg. Sur la base de nos recherches de terrain et de nos discussions avec les étudiants à l'université du Luxembourg, nous avons appelé ce phénomène « multiplurilinguisme». 
Concrètement, ce multiplurilinguisme luxembourgeois s'agence de la manière suivante dans le système éducatif luxembourgeois: au niveau précoce et préscolaire, un élève s'exprime d'abord en luxembourgeois, puis il ou elle se familiarise avec l'allemand, au moment de son alphabétisation à l'école fondamentale, vers 6 ans. Dans un troisième temps vient progressivement le français, à partir de l'âge de 8 ans. A ces trois langues principales se rajoute l'apprentissage de plusieurs langues vivantes: l'anglais, l'espagnol ou l'italien et d'autres langues encore. Traditionnellement, les langues de l'école reflétaient assez fidèlement les langues parlées dans les familles. Aujourd'hui, près de la moitié des enfants scolarisés au Luxembourg sont originaires de familles issues de l'immigration: par les langues et les traditions culturelles de leurs parents et grands-parents, les enfants apportent une richesse linguistique et culturelle que les enseignants apprennent à valoriser. Au niveau des enseignants, le multiplurilinguisme se montre dans le fait qu'ils sont de plus en plus enclins à prendre en compte la richesse linguistique et culturelle de leurs élèves, en valorisant toutes les langues présentes et en créant des liens entre elles, dans une approche d'intercompréhension et d'ouverture aux langues (Vasco Correia à paraître).

\subsection{Choix méthodologiques}

Pour notre étude sur la richesse linguistique luxembourgeoise, nous avons choisi de suivre l'orientation méthodologique de l'écologie linguistique. Tout comme l'écologie qui étudie le lien entre les éléments vivants dans un espace donné, l'écologie linguistique étudie le lien entre les langues, ou plutôt entre les personnes qui parlent certaines langues, à un endroit donné. L'écologie linguistique comporte ainsi une vision dynamique de la langue qui met l'homme et ses interactions sociales au premier plan. C'est un changement décisif par rapport à d'autres approches en sciences du langage qui avaient tendance à se concentrer sur les systèmes linguistiques et leur fonctionnement interne. Cette vision dynamique dépasse l'attitude de l'habitus monolingue qui a dominé presque partout et pendant une bonne partie du 20ème siècle (Bourdieu 1980; Gogolin 1994). Elle place l'être humain comme acteur au centre de l'environnement social qui crée des liens qui apportent du sens à ses mots (Pennycook/Makoni 2006). Plus récemment, l'écologie linguistique a trouvé une application dans l'environnement scolaire (Creese/Martin 2003) et promet de mettre en place des solutions nouvelles pour les défis auxquels l'actualité nous confronte. Elle étudie en effet le lien entre les langues de l'école et celles de la société qui l'entoure, et peut ainsi proposer des orientations pour la politique éducative et l'aménagement linguistique qui donneront les meilleures chances de réussite au plus grand nombre d'élèves, ce qui contribue à une plus grande cohésion sociale d'un pays ou d'une région. En résumé, l'écologie linguistique possède les traits suivants: 
- Elle est proche de la sociolinguistique et de l'étude du contact des langues.

- Son objectif est de considérer toutes les langues présentes à un endroit ou dans le répertoire d'une personne.

- Elle recherche une orientation équilibrée en évitant d'entrer dans un combat pour une langue ou la défense d'une autre langue.

- Elle observe les processus dynamiques ou fluides au lieu d'un état stable ou solide.

- Elle met l'être humain au centre de son intérêt.

\subsection{Bilan et orientations futures}

Nous avons suivi plusieurs classes au niveau préscolaire et primaire dans une étude portant au total sur cinq années et nous avons évalué l'apport des différentes approches en didactique plurilingue, pour faire le constat que la prise en compte de toutes les langues apportées par les élèves a été plus bénéfique pour tous les participants et pour toutes les langues que le fait de s'appuyer juste sur une seule. Trois années d'enseignement dans la formation des stagiaires de l'enseignement secondaire nous ont fait parvenir à la même conclusion. Cet effet de synergie n'est pas encore bien présent dans la littérature scientifique où l'on continue plutôt à argumenter dans le sens de la dynamique d'une seule langue sans regarder celles qui l'entourent. Les approches de l'éveil aux langues (surtout au niveau des jeunes élèves) et de la didactique du plurilinguisme au niveau des plus grands ont cette perspective holistique apportée par l'écologie des langues. La prise en compte peut se passer de manière plutôt symbolique pour certaines langues, à quelques moments bien précis dans la vie de l'école, comme les salutations, ou une chanson souhaitant «bon appétit » et " joyeux anniversaire " pour les élèves des premiers cycles de l'enseignement. Le fait que toutes les langues aient le droit de résonner dans le cadre de l'école libère la parole chez les enfants de toutes les classes d'âge, notamment pour l'apprentissage d'autres langues, et ceci sans distinction entre les langues officielles de l'école et les autres qui sont apportées grâce aux biographies variées des élèves. En résumé, nous voyons trois orientations centrales en ce qui concerne le développement d'un potentiel plurilingue chez les enfants (Leibenath/Ehrhart 2010): 
- les prédispositions des enfants comme l'âge, le talent et la motivation,

- le programme pédagogique des institutions éducatives,

- l'environnement familial et notamment son attitude par rapport à la pluralité linguistique et culturelle et aux institutions éducatives du pays où vit la famille.

Le plus grand succès dans le développement personnel d'un enfant se présente quand il y a une harmonisation entre ces différents domaines, quand l'évolution de l'élève correspond aux rythmes scolaires et quand les attentes familiales sont transparentes pour l'enfant et sans contradiction importante avec celles de l'institution scolaire. Ce cadre lui permettra de trouver sa voix et ainsi de devenir toujours davantage l'acteur de son propre destin.

La formation initiale et continue des enseignants occupe une place centrale dans cette démarche. Comme nous l'avons expliquée plus haut, outre l'observation sur le terrain, l'enquête par questionnaires et des interviews dirigés avec les enseignants et les enfants et leurs familles, les membres du projet LACETS intervenaient et interviennent toujours dans le Bachelor en Sciences de l'Education et dans la Formation Pédagogique à l'Université du Luxembourg ainsi que dans des séminaires de formation continue du SCRIPT (Service de Coordination de la Recherche et de l'Innovation Pédagogique et Technologique) du Ministère de l'Education Nationale. Les séminaires proposés portent sur la diversité linguistique et culturelle, l'écologie linguistique de la classe, l'ouverture aux langues, l'étude de ses propres attitudes et pratiques par une observation ethnographique, l'interdisciplinarité et l'hétérogénéité des stratégies d'apprentissage et d'enseignement. En tant que responsable du projet LACETS, j'étais également en contact avec les services de Ministère de l'Education Nationale (groupe d'experts pour le Plan d'Action Langues en collaboration avec les Institutions Européennes, groupe de travail sur l'ouverture aux langues avec le Service de Scolarisation des Enfants Etrangers). Ces contacts nous ont permis de communiquer et de vérifier les résultats de notre recherche.

La recherche au sein du groupe LACETS a certes donné des réponses à certaines questions, surtout en ce qui concerne la gestion de la classe plurielle, mais en même temps il a soulevé de nombreux autres questionnements, notamment en ce qui concerne l'acquisition naturelle d'une langue ou de plusieurs langues pendant l'enfance et l'adolescence. Le projet NaturaLink, un autre projet interne universitaire collectif sous ma direction et avec la collaboration de Sarah Vasco se penche sur cette question depuis 2011 et jusqu'en 2014 et assurera ainsi une continuité et une consolidation des acquis scientifiques de son projet précurseur.

A partir de septembre 2011 et dans le cadre de sa thèse de doctorat, Rahel Stoike étudie le plurilinguisme au sein de l'Université du Luxembourg, plus précisément dans des Masters plurilingues: le fait d'analyser les comportements 
dans l'espace plurilingue de notre environnement professionnel est important pour que nous nous rendions mieux compte de nos propres pratiques langagières au sein de notre institution, cette démarche nous rend plus crédibles vis-à-vis des autres institutions que nous sommes en train d'étudier dans notre recherche.

\section{$4 \quad$ Epilogue}

Après un début de parcours de chercheur plutôt solitaire en ce qui concerne l'accompagnement scientifique, mais en collaboration avec des tribus du Pacifique Sud, c'est depuis 15 ans environ que je participe activement à des projets internationaux. A chaque fois, c'est une expérience enrichissante et variée aussi bien sur le plan scientifique qu'humain. Les facteurs qui assurent le succès d'un projet sont la volonté de ses membres de travailler ensemble et de se décentrer dans une aventure interculturelle. Une bonne base administrative, ainsi qu'une durée de temps prédéfini et éventuellement prolongeable sont également des garants de qualité. Bien souvent, les appels à soumission des grands projets internationaux ne laissent pas assez de temps pour bien connaître ses partenaires et leurs objectifs, avant de se mettre en route ensemble. Il est souhaitable qu'une première phase de prise de contact ait déjà eu lieu avant de se lancer dans la rédaction d'un projet conjoint. LANGSCAPE est un lieu idéal pour rencontrer des partenaires solides et fiables avec lesquels nous sommes susceptibles de construire et déconstruire notre vision concernant l'identité et l'éducation des langues, dans un échange à la fois communautaire et respectueux de l'autre.

\section{Bibliographie}

Bourdieu, Pierre (1980). Le sens pratique. Paris: Minuit.

Byram, Mike / Dervin, Fred (2008). Students, Staff and Mobility in Higher Education. Newcastle: Cambridge Scholar Publishing.

Calvet, Louis-Jean (1999). Pour une Ecologie des Langues du Monde. Paris: Plon.

Creese, Angela / Martin, Peter (2003). Multilingual Classroom Ecologies: Interrelationships, Interactions and Ideologies. International Journal of Bilingual Education and Bilingualism 6/3-4: 161-167.

Ehrhart, Sabine (2012a). L'écologie des Langues de Contact: le tayo, créole de NouvelleCalédonie. Paris: L'Harmattan.

Ehrhart, Sabine (2012b). Der Umgang mit der sprachlichen Vielfalt in der Luxemburger Spillschoul. In: Mazurkiewicz-Sokołowska, Jolanta / Westphal, Werner / Gąszczyk, Alicja (éds.): Mehrsprachigkeit und Spracherwerb in Theorie und Praxis. Hamburg: Verlag Dr. Kovac. 63-74. 
Ehrhart, Sabine / Fehlen, Fernand (2011). Luxembourgish: A Success Story? A Small National Language in a Multilingual Country. In: Fishman, Joshua A. / García, Ofelia (éds.): Handbook of Language and Ethnic Identity - The Success-Failure Continuum in Language and Ethnic Identity Efforts. Volume II. Oxford: Oxford University Press. 285298.

Ehrhart, Sabine / Hélot, Christine / Le Nevez, Adam (éds.) (2010). Plurilinguisme et Formation des Enseignants: une approche critique. Plurilingualism and Teacher Education: A Critical Approach. Frankfurt/M.: Lang.

García, Ofelia / Bartlett, Lesley (2007). A speech community model of Bilingual Education: Educating Latino Newcomers. The U.S. International Journal of Bilingual Education and Bilingualism 10, 1-25.

García, Ofelia (2009). Bilingual Education in the 21st Century: a global perspective. New York: Wiley-Blackwell.

Gogolin, Ingrid (2008). Der monolinguale Habitus der multilingualen Schule. 2ième édition. Münster, New York: Waxman.

Ledegen, Gudrun (éd.) (2007). Pratiques linguistiques des jeunes en terrains plurilingues. Paris: L'Harmattan.

Leibenath, Renate / Ehrhart, Sabine (2010). Wie Grundschulen in Luxemburg auf die Sprachenvielfalt eingehen. Jeder kriegt eine Chance - praktische Beispiele für einen differenzierten Unterricht. Frühes Deutsch 21, 52-56.

Lüdi, Georges (1996). Mehrsprachigkeit. In: Goebl, Hans / Nelde, Peter H. / Starý, Zdeněk / Wölck, Wolfgang (éds.): Kontaktlinguistik. Ein internationales Handbuch zeitgenössischer Forschung. Volume 1. Berlin: de Gruyter. 320-327.

Markovina, Irina / Pisklakova, Svetlana / Zhura, Victoria (2013). Get Ready for Academic Mobility! How to participate in an International Conference: A Tutorial on Intercultural Communication for the Medical Profession. Moscow: Medical Information Agency Publication.

Musochranowa, Margarita / Ehrhart, Sabine (2008). Die Bestimmung pädagogischer Ausbildungsziele, Reihe „Ressourcen der Erde. Der Mensch.“ Beiträge der VI. regionalen Konferenz zur Wissenschaft, Praxis und Methodik. Persönlichkeit des Arztes im Kontext der Weltanschauung, Gesellschaft, Wissenschaft. Philosophie Zeitschrift OMSKI NAUČNYJ VESTNIK der Medizinischen Akademie Omsk (OmGMA): 107-113. (Traduction du russe à l'allemand par Maria Mushchinina, Dez. 2008), Manuscrit.

Pennycook, Alastair / Makoni, Sinfree (éds.) (2006). Disinventing and Reconstituting Languages. Clevedon: Multilingual Matters.

Perrefort, Marion (2008). Changer en échangeant: Mobilités et expériences langagières. In: Dervin, F. / Byram, Michael (éds.): Echanges et mobilités académiques - Quel bilan? Paris: L'Harmattan.

Trepos, Jean-Yves (2013). Arts de dire et formes de contrôle en situation de plurilinguisme. Université Ouverte des Humanités. Online: www.canal-u.tv/producteurs/canal_socio (dernier accès [26.7.2013]). 
Vasco Correia, Sarah (à paraître). La scolarisation peut-elle promouvoir un maintien et une revalorisation de la langue d'origine chez les enfants issus de l'immigration au Luxembourg? Présentation lors du colloque organisé par l'équipe PLURI-L à Angers en 2012. 



\title{
Englisch als europäische Lingua franca. Identitätsentwürfe, Erfahrungen und Einstellungen von Studierenden
}

\section{Claus Gnutzmann / Jenny Jakisch / Frank Rabe (Braunschweig)}

\begin{abstract}
With English usually being the most important language in encounters between Europeans who do not share the same mother tongue, the process of identity construction of young Europeans is not only influenced by their first languages, but increasingly affected by the wide use of English as a lingua franca (ELF) in Europe or, respectively, Euro-English. Against this backdrop, the European Union's attempts to foster the development of a common European identity on the basis of language diversity and individual plurilingualism raise the question as to what extent young Europeans' identity formation takes place under the impact of ELF. Drawing on an analysis of 620 answers to an open question from a questionnaire survey conducted at the University of Braunschweig (Germany), the article investigates how students conceptualize identity with regard to different forms of English.

L'anglais étant habituellement la langue la plus répandue dans les rencontres entre Européens de langue maternelle différente, le processus de construction de l'identité des jeunes Européens n'est pas seulement influencé par leur première langue, mais elle est de plus en plus affectée par l'usage de l'anglais en tant que lingua franca (ELF) ou encore 1'Euro-English. Sur cette trame, les efforts de l'Union européenne afin de promouvoir le développement d'une identité européenne commune - reposant sur la diversité des langues et le plurilinguisme - soulèvent la question de déterminer à quel point la formation de l'identité des jeunes Européens est influencée par l'ELF. Se fondant sur l'analyse de 620 réponses libres à un questionnaire d'étude effectué à l'Université de Braunschweig (Allemagne), l'article examine la façon dont les étudiants conceptualisent leur identité à travers leur perception des différentes formes d'anglais.
\end{abstract}

\section{$1 \quad$ Einleitung}

Es ist allgemein bekannt, dass das Englische nicht nur weltweit, sondern auch innerhalb Europas zur beliebtesten language of choice für die Kommunikation unter Europäern, die nicht dieselbe Muttersprache teilen, avanciert ist. Seine extensive Verwendung als europäische Lingua franca in interkulturellen Begegnungssituationen entspricht allerdings nicht den sprachenpolitischen Vorstellungen der Europäischen Union, die zumindest offiziell Kenntnisse in einer Fremdsprache nur bedingt als ausreichend für die wechselseitige Verständigung unter Europäern und die Entwicklung einer europäischen Identität erachtet. Vielmehr postuliert die EU das Konzept der individuellen Mehrsprachigkeit, verstanden als Kompetenz in der Muttersprache und das Verfügen über (ausbau- 
fähige) Kompetenzen in mindestens zwei, vorzugsweise europäischen Sprachen. Des Weiteren geht sie davon aus, dass sich so die für Europa charakteristische Situation der sprachlichen und kulturellen Vielfalt erhalten lässt, auf deren Grundlage das Zusammenwachsen Europas einschließlich der damit verbundenen Identitätsentwicklung angebahnt werden kann. ${ }^{1}$ Dass dieses Kommunikationsmodell in Konkurrenz zur Nutzung des Englischen als europäischer Lingua franca tritt, wie es insbesondere für junge Europäer längst zum Alltag gehören dürfte, lässt allerdings vermuten, dass nicht nur das Mit- und Nebeneinander vieler Sprachen, sondern vor allem der Gebrauch einer gemeinschaftlich geteilten Sprache den Austausch unter Europäern erleichtern und zum Verständnis der anderssprachigen Nachbarn beitragen kann.

Der vorliegende Beitrag widmet sich der Frage, welche Rolle das Englische speziell in seiner Funktion als europäische Lingua franca sowie in seiner möglichen Ausprägung als Euro-English - im Prozess der europäischen Identitätsentwicklung einnehmen kann. Ausgehend von Überlegungen zum Identitätsbegriff in seiner individuellen und europäischen Dimension (Abschnitt 2) sowie einer Erörterung des Potenzials von (Euro-)Englisch als mögliche Quelle für eine europäische Identität (Abschnitt 3) werden dazu Daten aus einer an der Technischen Universität Braunschweig durchgeführten Fragebogenstudie mit Studierenden des 1. und 2. Studienjahres ${ }^{2}$ diskutiert (Abschnitt 4). Neben quantitativen Ergebnissen bieten vor allem die Analysen der erhobenen Freitexte der Teilnehmer zur Frage, inwiefern Euro-Englisch dazu beitragen könnte, eine eigene europäische Identität $\mathrm{zu}$ schaffen, Einblicke in die Identitätsentwürfe junger Europäerinnen und Europäer und ermöglichen eine Rekonstruktion ihrer diesbezüglichen Erfahrungen und Einstellungen. Der Beitrag schließt mit einer Zusammenfassung und Perspektivierung der Ergebnisse (Abschnitt 5).

\section{Identitätsentwicklung im europäischen Kontext}

Im Zeitalter von Globalisierung, medialer Vernetzung und zunehmender Individualisierung unterliegen Identitätsentwürfe junger Europäer vielfältigen, nicht an nationale Grenzen gebundenen Einflussfaktoren. Identitäten sind dynamisch, mehrschichtig und komplex (vgl. z. B. Faez 2011); insbesondere der Sprache ist eine zentrale Rolle im Prozess der Identitätsbildung zu attestieren (vgl. Norton/Toohey 2011: 413). Folgt man Nortons Auslegung, so gibt der Identitätsbegriff Auskunft darüber, ,how people understand their relationship to the

\footnotetext{
${ }^{1}$ Vgl. hierzu auch das seit 2000 existierende Europamotto „In Vielfalt geeint“, das die sprachliche und die kulturelle Vielfalt der EU thematisiert.

2 Wir bedanken uns sehr herzlich bei allen Studierenden und Lehrenden der TU Braunschweig, die an der Erhebung kooperativ und mit Interesse mitgewirkt haben.
} 
world, how that relationship is constructed across time and space, and how people understand their possibilities for the future" (Norton 1997: 410). Diese Aushandlung zwischen 'Ich' und 'Welt' findet insbesondere über Sprache statt, und sie umfasst sowohl eine individuelle als auch eine kollektive Komponente.

Block (2007: 43) versteht Identität als ein Konstrukt, das aus verschiedenen Perspektiven, wie beispielsweise Klassenzugehörigkeit, Gender oder nationalen Verortungen, betrachtet und analysiert werden kann. In diesem Zusammenhang beschreibt er sprachliche Identität als „,relationship between one's sense of self and different means of communication, understood in terms of language, a dialect or sociolect, as well as multimodality“. Letzteres wird von ihm gewählt, um $\mathrm{zu}$ verdeutlichen, dass über das verwendete sprachliche Zeichensystem hinaus sowohl die räumliche wie auch die gesellschaftlich-soziale Positionierung einen Einfluss auf die Art der Identitätsaushandlung hat: „Clearly, where and how one is physically situated is a big part of who one is" (ebd.: 44).

Die Fähigkeit zu sprachlichem Handeln wird zunächst über die Erstsprache erworben, differenziert sich aber über zusätzliches, institutionelles und lebensweltliches Sprachenlernen weiter aus. Im Fall des Englischen als am häufigsten angebotener erster schulischer Fremdsprache im deutschen Kontext ist davon auszugehen, dass der zu lernenden Sprache vom Großteil der Lernenden Interesse entgegengebracht und von Seiten der Gesellschaft ein hohes 'kulturelles Kapital' unterstellt wird. Daher kann vermutet werden, dass die erworbenen Kenntnisse als Bereicherung des eigenen sprachlichen Handlungsrepertoires empfunden und somit als positive Erweiterung der eigenen Identität wahrgenommen werden. Die Begegnung mit anderen Sprachen, wie sie über den schulischen Fremdsprachenunterricht angeregt wird, bleibt somit nicht ohne Folgen für die Identitätsentwicklung der Lernenden. Sie kann aber auch zu Abwehrreaktionen und einer Distanzierung von der fremden Sprache und der mit ihr assoziierten Kultur führen, vor allem dann, wenn sie weniger erfolgreich ist oder prägende Erfahrungen, die der Lernende nicht einzuordnen vermag, vorliegen.

Auch die Identitätsentwicklung von Studierenden unterliegt vielfältigen (fremd-)sprachlichen Einflussfaktoren. Sie findet nicht mehr nur in den jeweiligen National- bzw. Erstsprachen statt, sondern wird zunehmend dadurch beeinflusst, dass den Studierenden Mehrsprachigkeit in verschiedenen Kontexten, beispielsweise durch die kontinuierlich steigende Anglifizierung von Forschung und Lehre, begegnet. Nicht zuletzt vor dem Hintergrund der zunehmenden Bedeutung des Englischen in seiner Funktion als Lingua franca in Europa (vgl. Jenkins 2007; Seidlhofer 2011a) sollten sie daher in der Lage sein, in mehreren Sprachen zu agieren, sodass sie sich selbst nicht nur in einsprachigen Handlungsfeldern erleben, sondern neue Facetten der eigenen Persönlichkeit entdecken können, wenn die Kommunikation über eine bzw. mehrere Fremdsprachen verläuft. 
Es liegt auf der Hand, dass Identität vor diesem Hintergrund nicht als feststehende, mit einem bestimmten Zeitpunkt in der Persönlichkeitsentwicklung abgeschlossene Größe verstanden werden kann. Vielmehr scheint die Vorstellung eines pluriellen, sich in der Auseinandersetzung mit der (fremd-) sprachlichen Umwelt stetig weiterentwickelnden, dynamischen Konstrukts treffender: „At any given time a person's identity is a heterogeneous set made up of the names or identities, given to and taken up by her" (Tabouret-Keller 1997: 316). Da sprachliches Handeln im Austausch mit anderen erfolgt und auf erwartbaren, gemeinschaftlich geteilten Interaktionsmustern basiert, umfasst Identität immer eine individuelle und eine kollektive Dimension. Letztere bezieht sich sowohl auf gesellschaftliche und nationale Gefüge, meint aber im Kontext des zusammenwachsenden Europas auch, dass „sich Menschen in Europa kognitiv und emotional mit Europa als einem abgrenzbaren Raum verbunden fühlen“ (Nissen 2004: 21). Die Ergebnisse der Eurobarometer-Studie legen nahe, dass eine solche Verbundenheit existiert: 57\% der Deutschen gaben an, sich 'manchmal' oder 'oft' als Europäer zu fühlen, europaweit sind es im Vergleich 54\% (vgl. Europäische Kommission 2007: 37). ${ }^{3}$ Der Wunsch nach einem „europäischen Patriotismus“ (Gauck 2013), wie ihn der deutsche Bundespräsident kürzlich bei einer Rede formuliert hat, zeigt jedoch, dass eine europäische Identität erst im Entstehen begriffen ist und ihre Eckpunkte derzeit noch eher vage sind. ${ }^{4}$

\section{3 (Euro-)Englisch versus Mehrsprachigkeit als Grundpfeiler europäischer Identität}

Die EU hat sich offiziell dem Erhalt und der Förderung der kulturellen und sprachlichen Vielfalt in Europa verschrieben und vertritt die Auffassung, dass auf der Grundlage einer individuellen Mehrsprachigkeit der Unionsbürger der Austausch über Sprachgrenzen hinweg ermöglicht, die Mobilität innerhalb Europas gestärkt und die Entwicklung einer gemeinsamen europäischen Identität begünstigt werden können. Dass insbesondere die ersten beiden Funktionen

\footnotetext{
${ }^{3}$ Den Daten des Eurobarometers 75 (Europäische Kommission 2011: 35f.) zufolge erweisen sich die Reisefreiheit (45\%) sowie der Euro (38\%) als zentrale gemeinsame Bezugspunkte der Europäer. Aber auch Geldverschwendung, Frieden, mehr Mitsprache in der Welt, Bürokratie, kulturelle Vielfalt und Demokratie (24-20\%) sind Vorstellungen, die die EU-Bürger mit Europa assoziieren.

${ }^{4}$ Zur Verwirklichung des Prinzips Mehrsprachigkeit hebt der Bundespräsident vor allem auf die Verwendung der Muttersprache und des Englischen ab: „Ich bin überzeugt, dass in Europa beides nebeneinander leben kann: die Beheimatung in der eigenen Muttersprache und in ihrer Poesie und ein praktikables Englisch für alle Lebenslagen und Lebensalter" (Gauck 2013: 11).
} 
häufig vom Englischen übernommen werden, das sich längst zur faktischen Lingua franca Europas entwickelt hat, wird allerdings von der EU nur bedingt anerkannt und teilweise sogar tabuisiert (vgl. Gnutzmann 2008: 21; House 2006: 91; Seidlhofer 2011b: 134). Die von der EU präferierte Gleichberechtigung aller europäischen Sprachen, von denen das Englische nur eine ist, hat in der Realität jedoch kaum Bestand, denn ,it is common knowledge that some languages in the European context are more equal than others" (House 2008: 64). Zudem scheinen Kompetenzen in mehreren Sprachen wenig hilfreich, wenn das Gegenüber diese nicht teilt oder wenn man mit einem Sprecher der vielen anderen europäischen Staaten kommunizieren will, dessen Sprache man selbst nicht beherrscht (vgl. Seidlhofer 2011b: 134). Angesichts der Vielzahl europäischer Sprachen erscheint - zumindest auf den ersten Blick - die Möglichkeit, auf eine Sprache wie das Englische zurückgreifen zu können, daher weitaus erfolgversprechender. Ob die Sorge vor einer zunehmenden Dominanz angloamerikanischer Werte und der drohenden Übermacht des Englischen über das Konzept der europäischen Mehrsprachigkeit zerstreut werden kann, ist daher fraglich. ${ }^{5}$

Allerdings hat die Verwendung des Englischen als paneuropäisches Kommunikationsmittel auch Nachteile. So müssen beispielsweise Nichtmuttersprachler z. T. erheblichen Aufwand betreiben, um die benötigten Kompetenzen im Englischen $\mathrm{zu}$ erwerben. Ferner kann der raumgreifende Gebrauch des Englischen, wie er beispielsweise in den Fachdiskursen der Wissenschaften immer stärker voranschreitet, zu Domänenverlusten in den jeweiligen Muttersprachen führen. Hinzu kommt, dass die Nutzung einer gemeinsamen Sprache innerhalb Europas dem plurilingualen und mehrkulturellen Charakter Europas nicht ausreichend Rechnung tragen würde. So argumentierte Mundschau (1995: 345) schon vor fast zwanzig Jahren:

Die Sprache ist [...] der Schlüssel zur europäischen Einigung. Der Wille dahin wird an der Qualität des zukünftigen Sprachverhaltens gemessen. Eine europäische Einheitssprache wird es niemals geben. Sprache bedeutet Identität, und mit dem Verlust der eigenen Identität möchte niemand zum Europäer werden.

Die besondere sprachliche Situation innerhalb Europas wirft daher die Frage auf, ob sprachliche Unterschiede, wie im Mehrsprachigkeitsansatz der europäischen Sprachenpolitik verankert, identitätsstiftend sein können oder ob nicht vielmehr sprachliche Gemeinsamkeiten, wie sie zum Beispiel der Gebrauch des Englischen als europäische Lingua franca herstellen könnte, die Basis für eine europäische Identität bilden. Gerade junge Europäer, in deren

5 Weiterführende Überlegungen für eine Fortentwicklung europäischer Sprachen- und Bildungspolitik im Hinblick auf das Leitziel Mehrsprachigkeit finden sich bei Küster (2007: 45f.). 
Leben das Englische eine wachsende Bedeutung einnimmt und die häufig im Alltag davon umgeben sind, dürften sich, entgegen der im vorangehenden Zitat zum Ausdruck gebrachten Auffassung, weniger von einem Sprach- und damit verbundenen möglichen 'Identitätsverlust' durch das Englische betroffen sehen. Eher ist zu vermuten, dass sie - vermutlich ohne sich dessen bewusst zu sein ihre Identität schon längst zu einem Teil durch die englische Sprache definieren.

Es wäre daher zu überlegen, ob die Rolle des Englischen als europäische Verkehrssprache aus (sprachen-)politischen Erwägungen weiterhin eingeschränkt oder ob ihre de facto-Position nicht nur toleriert, sondern sogar unterstützt werden sollte. Ein identitätsstiftendes Potenzial könnte insbesondere von einem sogenannten Euro-English ausgehen, d. h. einer Form des Englischen, die in Europa gesprochen wird und sich bezüglich Aussprache, Grammatik und Wortschatz vom amerikanischen und britischen Englisch unterscheidet. So nehmen einige Forscher an, dass ELF nicht nur Medium der paneuropäischen Kommunikation ist, sondern von den Nutzern unter Verzicht auf die inner circle-Normen englischer Muttersprachler (Kachru 1985) weiterentwickelt und eigenständig geprägt wird.

Anhand der Daten des VOICE-Korpus (Vienna Oxford International Corpus of English) stellten beispielsweise Seidlhofer und ihr Team fest, dass ELFVerständigung von bestimmten strukturellen Merkmalen und Formulierungen gekennzeichnet ist, „which by native-speaker standards would be 'errors' but are generally unproblematic and no obstacle to communicative success" (Seidlhofer 2005: s. p.). Dazu zählen u. a. die Reduzierung der Verbkonjugation durch Verzicht auf das third-person singular present tense $-s$ (she like) oder die Pluralbildung für Nomen, die im Standardenglischen keine Mehrzahl haben (informations, advices) (vgl. ebd.). Zudem kann bereits beobachtet werden, dass gängige Ausdrücke aus den europäischen Sprachen durch den Prozess der discoursal nativization (Modiano 2001: 14), d. h. die kommunikative Akzeptanz vormals als inkorrekt erachteter Strukturen und Ausdrucksweisen, Eingang in das in Europa gesprochene Englisch gefunden haben. Der Annahme widersprechend, dass ELF als ein „'native-culture-free' code“ (Pölzl 2003: 5) angesehen werden kann, vertritt Fiedler (2011: 86f.) die Position, dass insbesondere fortgeschrittene ELF-Sprecher sich auch anglophon-muttersprachliche Phraseologie und kulturspezifische Wendungen zu eigen machen. ${ }^{6}$ Indizien wie diese untermauern die Annahme, dass ELF-Sprecher keine 'kulturlose', streng funktionale Kommunikation praktizieren, sondern durchaus Komponenten der

6 Fiedler (2011: 86) verweist unter anderem auf das folgende, auf einen englischen Hochzeitsspruch Bezug nehmende Beispiel eines norwegischen Sprechers: "In my paper you will find something old (.) something new something borrowed (.) something blue (.) let me start by ...." 
anglophonen Kulturen und ebenso Bestandteile ihres eigenen kulturellen Hintergrundes in die Nutzung des Englischen als Lingua franca einfließen lassen.

$\mathrm{Ob}$ Euro-Englisch jedoch den Status einer eigenen Varietät für sich beanspruchen kann, ist ebenso umstritten wie die Frage, ob dies überhaupt von den Europäern akzeptiert wird oder werden könnte. So argumentiert beispielsweise James ( $\left.{ }^{2} 2008: 140\right)$, dass sich die Europäer an den Standardvarietäten des britischen und amerikanischen Englisch orientieren und somit Belege für die Entwicklung eines Euro-English fehlen. Modiano (2003: 35) gibt diesbezüglich zu bedenken, dass ein Euro-Englisch nicht als eigenständige Form des Englischen anerkannt würde, solange ein Muttersprachlerideal als Vorbild das Englischlernen in Europa dominiert und damit eine Sprachenhierarchie besteht. Auch empirische Belege für die Existenz von Euro-Englisch als endonormative Varietät sind rar, sodass Mollin (2006: 1) festhält: „Euro-English seems to be the Yeti of English varieties: everyone has heard of it, but no one has ever seen it." Wenngleich sich also das in Europa gesprochene Englisch vom britischen und amerikanischen Englisch unterscheidet, kann der Prozess zur Bildung und besonders zur Akzeptanz eines Euro-Englisch - sollte es denn existieren derzeit bestenfalls als in den Anfängen befindlich beschrieben werden.

Ungeachtet dessen, ob es ein solches Euro-Englisch bereits gibt, könnte in ihm ein besonderes identitätsstiftendes Potenzial liegen. Eine eigene Form des Englischen in Europa, die von Nichtmuttersprachlern geformt wird, wäre also ein möglicher Lösungsweg aus dem Dilemma, dass einerseits eine gemeinsame Sprache einer europäischen Identität zuträglich wäre, andererseits aber eine Nationalsprache als einzige Verkehrssprache in Europa als Bedrohung der Mehrsprachigkeit gesehen wird (vgl. Seidlhofer 2011b: 133). Euro-English böte die Möglichkeit, zweitsprachliche Identitäten zu schaffen, die den kulturellen und muttersprachlichen Hintergrund der Einwohner der verschiedenen Mitgliedsländer berücksichtigen. Grundlage dafür wäre jedoch, dass es von einer Mehrheit der Europäer als eigenständige Form des Englischen anerkannt und verwendet wird. Dafür müsste es aber auch in prestigeträchtigen Kontexten, beispielsweise als Unterrichts- und Behördensprache, gebraucht werden (vgl. Gnutzmann 2008: 30). Dies ist allerdings insofern problematisch, als EuroEnglish bzw. eine europäische Lingua franca Englisch häufig mit mangelnder Sprachbeherrschung in Verbindung gebracht wird. Es wäre daher zu untersuchen, ob Englisch in Europa in absehbarer Zeit eine eigenständige Sprache werden kann, die ihren mehrsprachigen Sprechern 'gehört' und von diesen geformt wird, oder ob die Orientierung an muttersprachlichen Normen trotz zunehmender paneuropäischer Kommunikation dominant bleiben wird. 


\section{Die empirische Erhebung}

\subsection{Methodisches Vorgehen}

Um der Frage nachzugehen, wie junge Europäer den Einfluss von ELF auf ihre Identitätsbildung wahrnehmen, wurden in einer im Wintersemester 2011/12 durchgeführten empirischen Erhebung an der Technischen Universität Braunschweig 1061 Studierende aus den Fachrichtungen Anglistik, Germanistik, Lebenswissenschaften und Maschinenbau im 1. und 2. Studienjahr befragt. Vor dem Hintergrund der Tatsache, dass die meisten Studierenden mit dem Englischen vertraut sein dürften, ist zu vermuten, dass sich ein Einfluss der englischen Sprache auf eine europäische Identität am deutlichsten bei jungen Europäern und damit der gewählten Zielgruppe zeigt. Mit Hilfe des für die Untersuchung gewählten Instrumentes, eines aus 22 geschlossenen und einer offenen Frage bestehenden Fragebogens ${ }^{7}$, konnten Daten von einer großen Anzahl Teilnehmender erhoben werden.

Inbesondere die Nutzung der den Fragebogen abschließenden offenen Frage ermöglichte es, Begründungen von den Studierenden dazu einzufordern, ob ein eigenständiges, sich vom britischen und amerikanischen Englisch unterscheidendes Euro-Englisch dazu beitragen könnte, eine eigene europäische Identität zu schaffen. Zur Auswertung der Daten mussten zunächst sämtliche von den Studierenden verfassten Freitexte transkribiert werden (vgl. Tab. 1).

Tabelle 1: Ausfüllstatistik - Verhältnis der ausgefüllten Fragenbögen zu verfassten Freitexten

\begin{tabular}{|l|c|c|c|}
\hline \multicolumn{1}{|c|}{ Fach } & $\begin{array}{c}\text { Anzahl } \\
\text { Fragebögen }\end{array}$ & $\begin{array}{c}\text { Anzahl } \\
\text { Freitexte }\end{array}$ & $\begin{array}{c}\text { Freitext } \\
\text { geschrieben in \% }\end{array}$ \\
\hline Anglistik & 164 & 146 & $89 \%$ \\
\hline Germanistik & 250 & 182 & $73 \%$ \\
\hline Lebenswissenschaften & 231 & 111 & $48 \%$ \\
\hline Maschinenbau & 416 & 240 & $58 \%$ \\
\hline GESAMT & $\mathbf{1 0 6 1}$ & $\mathbf{6 7 9}$ & $\mathbf{6 4 \%}$ \\
\hline
\end{tabular}

Wie aus der Tabelle ersichtlich wird, haben 679 Studierende einen Freitext verfasst, wobei prozentual weniger Personen in den Lebenswissenschaften und im Maschinenbau Freitexte geschrieben haben als dies in den Fächern Germanistik und insbesondere Anglistik der Fall war. Dies könnte damit zusammenhängen, dass Studierende der beiden letzteren Fächer einen stärkeren Bezug zum Thema bzw. zu sprachlichen Fragen generell haben. Nicht themenspezifische Aussagen und Freitexte, in denen sich keinerlei Begründung fand

\footnotetext{
${ }^{7}$ Für eine detaillierte Auswertung der geschlossenen Fragen siehe Gnutzmann et al. (2012).
} 
( $\mathrm{n}=59$, z. B. „Ist halt so“), wurden nicht weiter berücksichtigt. Grundlage für die weitere Auswertung waren die verbleibenden 620 Freitexte.

Um kollektiv geteilte Begründungsmuster und unter den Befragten vorherrschende Einstellungen herausarbeiten zu können, galt es, die Fülle an Daten durch Kodieren zu systematisieren. Dabei wurde davon ausgegangen, dass die von den Studierenden verfassten Antworten als eigenständige Texte interpretierbar sind und somit Aufschluss über zugrunde liegende Wahrnehmungen und Einstellungen zum Thema (Euro-)Englisch und Identität geben können. Im Kodiervorgang selbst wurde zuerst ein Sample des Freitextkorpus, das 10\% aller Freitextantworten unter Berücksichtigung aller Studienrichtungen enthielt, induktiv kodiert, d. h., es wurde eine ,Zuordnung von Kategorien zu relevanten Textpassagen“ (Kuckartz 2010: 57) vorgenommen. Dabei wurden inhaltliche Überlappungen in den Codes durch die Vergabe von Ober- und Untercodes verdeutlicht. Der so entstandene Kodierbaum wurde anschließend auf die restlichen Daten angewandt; neue Codes wurden immer dann eingeführt, wenn eine Aussage sich nicht den bereits existierenden Codes zuordnen ließ. Diese 'Schleife' wurde solange wiederholt und im Forscherteam besprochen, bis keine neuen Zweifelsfälle mehr auftauchten und Übereinstimmung unter den Teammitgliedern erreicht werden konnte. Insgesamt wurden den 620 Freitexten 998 Codes zugeordnet, da in einem Text häufig mehrere Themen angesprochen wurden. Diese wurden wiederum zu sieben Themenfeldern gebündelt (vgl. Tab. 2), die im Folgenden nach Häufigkeit der Zuordnungen präsentiert werden.

Tabelle 2: Anzahl der Zuordnungen zu den einzelnen Themenfeldern

\begin{tabular}{|l|c|}
\hline \multicolumn{1}{|c|}{ Themenfeld } & $\begin{array}{c}\text { Anzahl der } \\
\text { Zuordnungen }\end{array}$ \\
\hline Sprache und Identität & 396 \\
\hline Praktikabilität von Euro-Englisch & 175 \\
\hline Wahrgenommene Eigenschaften von Euro-Englisch & 145 \\
\hline Euro-Englisch und Kommunikation & 115 \\
\hline Abgrenzung und Gemeinsamkeit & 64 \\
\hline Alternative Forderungen & 54 \\
\hline Wem gehört das Englische? & 49 \\
\hline
\end{tabular}

Bei der Auswertung der Freitexte ergab sich ein Spannungsverhältnis zwischen den Ansprüchen qualitativer sowie eher quantitativer Ansätze. Einerseits ging es vor allem darum, Identitätsentwürfe der Studierenden sowie ihre Verbindung zu Sprache zu beschreiben, weshalb auch zahlenmäßig vernachlässigbare Codes bei der Auswertung berücksichtigt wurden. Andererseits erschien es ebenfalls interessant, Häufigkeitsaussagen über bestimmte Codes treffen zu können, da dies Anhaltspunkte hinsichtlich der Verbreitung der herausgearbeiteten Auffas- 
sungen gibt. Es wurde deshalb der Versuch unternommen, diese Facetten nutzbringend zu verbinden.

\section{2 $\quad$ Ergebnisse}

Im geschlossenen Teil des Fragebogens, der Antwortoptionen auf einer vierstufigen Likert-Skala vorgab, wurden die Studierenden u. a. um eine Einschätzung der bestehenden Varietäten des Englischen gebeten. Dabei zeigte sich, dass 91\% der Befragten Unterschiede (z. B. bezüglich Aussprache, Grammatik und Wortschatz) zwischen dem von Muttersprachlern und Nichtmuttersprachlern gesprochenen Englisch wahrnahmen, daraus aber nicht den Anspruch ableiteten, eine eigene Form des Englischen innerhalb Europas zu benutzen. Im Gegenteil: Insgesamt 95\% der Teilnehmer lehnten die Aussage ab, dass es in Europa eine eigene Form der englischen Sprache (z. B. Euro-Englisch) geben sollte und bevorzugen stattdessen eindeutig die Orientierung an native speaker-Standards. Es verwundert daher nicht, dass mit 84\% die deutliche Mehrheit der Studierenden die Frage verneint, ob ein solches Euro-Englisch dazu beitragen könne, eine eigene europäische Identität zu schaffen. Aufschluss über mögliche Gründe für diese überwiegende Ablehnung gibt die im Folgenden vorzustellende Auswertung des Freitextkorpus.

\section{Sprache und Identität}

Das erste und zahlenmäßig größte der sieben gebildeten Themenfelder bündelt neun Codes (vgl. Tab. 3, s. unten), die u. a. Aussagen zur Identitätsbildung durch Euro-Englisch (EE) oder durch Nationalsprachen, zur Mehrsprachigkeit in Europa und zur Rolle außersprachlicher Faktoren im Prozess der Identitätsentwicklung enthalten.

Tabelle 3: Codes im Themenfeld „Sprache und Identität“

\begin{tabular}{|c|c|c|}
\hline Obercode (O)/Untercode (U) & \multicolumn{2}{|c|}{ Anzahl/Gesamt } \\
\hline $\mathrm{EE}=$ identitätsstiftend $(\mathrm{O})$ & 66 & \multirow{2}{*}{79} \\
\hline - $\quad$ Nicht-muttersprachliches Englisch schafft europäische Identität (U) & 13 & \\
\hline Identität über Sprachenvielfalt und kulturelle Vielfalt (O) & 71 & \multirow{2}{*}{125} \\
\hline - Identität wird über Nationalsprache und -kultur gestiftet (U) & 54 & \\
\hline EE nicht als alleiniges Fundament für europäische Identität (unbestimmt) $(\mathrm{O})$ & 57 & \multirow{2}{*}{70} \\
\hline - Identität durch Gemeinsamkeiten außerhalb von Sprache (U) & 13 & \\
\hline $\mathrm{EE} \neq$ identitätsstiftend & \multicolumn{2}{|c|}{99} \\
\hline Eine europäische Identität ist bereits vorhanden & \multicolumn{2}{|c|}{10} \\
\hline Europäische Identität wird es nie geben & \multicolumn{2}{|c|}{13} \\
\hline
\end{tabular}


66 Studierende bringen im ersten Obercode zum Ausdruck, dass Euro-Englisch eine identitätsstiftende Wirkung entfalten könne (z. B. „Ich stimme schon zu, dass es eine eigene Identität schaffen würde, weil Europa dann sozusagen etwas ,eigenes ' ist" $\left.[17 \mathrm{~A}]^{8}\right)$. Im dazugehörigen Untercode $(\mathrm{n}=13)$ wird zusätzlich zur obigen Einstellung darauf hingewiesen, dass eine identitätsstiftende Wirkung bereits beim Gebrauch von ELF vorliegt, ohne dass es dazu einer eigenen Form des Englischen bedürfe. Dass eine gemeinsame Sprache für Europa identitätsstiftend wirken könnte, kommt darüber hinaus im Obercode „EE nicht als alleiniges Fundament für europäische Identität“ $(n=57)$ zum Ausdruck. Allerdings wird hier die identitätsstiftende Wirkung von Sprache dahingehend eingeschränkt, dass auch nichtsprachliche Elemente zu einer Identitätsbildung beitragen. Dieses Ergebnis spiegelt sich ebenso im Fragebogen bei der Frage „Was ist für Sie typisch europäisch?" wider, bei der die Befragten aus einer Liste mit 15 vorgegebenen Items drei Nennungen auswählen sollten. Die erhaltene Rangliste (1: der Euro; 2: Reisefreiheit; 3: Demokratie; 4: Sprachenvielfalt; 5: Menschenrechte) verdeutlicht, dass für die Gruppe bei der Bestimmung von 'Eckpunkten' einer europäischen Identität nichtsprachliche Werte im Vordergrund stehen.

Es überwiegen in diesem Themenfeld allerdings Äußerungen, in denen die Auffassung zum Ausdruck kommt, dass Euro-Englisch nicht identitätsstiftend wirken könne ( $\mathrm{n}=99$; z. B. „Euro-Englisch würde sich nicht durchsetzen, geschweige denn eine europäische Identität schaffen" [10G]). Eine Rekonstruktion der Einstellungen lässt zudem deutlich werden, dass einige Befragte Englisch ausschließlich als Instrument zur Kommunikation sehen, das in keinerlei Zusammenhang zur Identitätsentwicklung steht (,Sprachen sind nur Werkzeuge zur Kommunikation und tragen für mich nicht $\mathrm{zu}$ irgendeiner Art von ,Identität' bei“" [323L]). Außerdem sind einige Befragte der Meinung, dass eine europäische Identität bereits vorhanden sei $(n=10)$, was die Einführung von Euro-Englisch hinfällig mache, oder sie widersprechen der Annahme, dass es überhaupt eine europäische Identität geben könne $(\mathrm{n}=13)$.

Mit insgesamt 125 Zuordnungen dominieren die nächsten beiden Codes, ein Ober- und Untercode, von der Anzahl her das Themenfeld. Obwohl die Codes auf den ersten Blick disparat wirken, können sie als zwei Seiten derselben Medaille verstanden werden: Der Obercode „Identität über Sprachenvielfalt und kulturelle Vielfalt" $(\mathrm{n}=71)$ thematisiert zwar den Wunsch nach Mehrsprachigkeit, häufig wird darunter aber die mosaikartige Koexistenz verschiedener Nationalsprachen und -kulturen innerhalb der EU verstanden (vgl. Gal 2011: 24)

\footnotetext{
${ }^{8}$ Die Zahlen verweisen auf die jeweiligen Texte im Freitextkorpus, die Buchstaben auf die Fachgebiete: A steht für Anglistik, G für Germanistik, L für Lebenswissenschaften und M für Maschinenbau. Die Beispielsätze sind unter Erhalt der Originalorthografie aus dem Korpus übernommen.
} 
und nicht das individuelle Verfügen über mehrere Fremdsprachen („Die europäische Identität ergibt sich aus den verschiedenen europäischen Sprachen und Kulturen“ [19A]). In diesem Sinne erfasst der Untercode „Identität wird über Nationalsprache und -kultur gestiftet" $(\mathrm{n}=54)$ nur eine andere Facette derselben Botschaft: Identitätsstifend ist die Nationalsprache, diese reiche aus, um 'Vielfalt' in Europa zu gewährleisten („Eine neue Form des Englischen würde sich nicht durchsetzen, weil jedes Volk sich anhand seiner eigenen Sprache identifizieren will“" $[3 \mathrm{G}])$.

\section{Praktikabilität von Euro-Englisch}

Dieses Themenfeld vereint Codes, die die Effizienz oder Umsetzbarkeit verschiedener Formen des Englischen thematisieren und damit überwiegend pragmatische Gesichtspunkte betonen (vgl. Tab. 4).

Tabelle 4: Codes im Themenfeld „Begründete Ablehnung von Euro-Englisch“

\begin{tabular}{|l|c|c|}
\hline \multicolumn{1}{|c|}{ Obercode/Untercode } & \multicolumn{2}{c|}{ Anzahl/Gesamt } \\
\hline Vorhandene englische Sprache praktischer/ausreichend & \multicolumn{2}{|c|}{110} \\
\hline Umsetzbarkeit von EE schwierig (ohne weitere Begründung) & 29 & \multirow{2}{*}{65} \\
\hline • EE verursacht erhöhte Lernaufwand oder Lernprobleme & 36 & \\
\hline
\end{tabular}

Euro-Englisch wird mit der Begründung abgelehnt, dass die bereits existierenden englischen Varietäten praktischer bzw. ausreichend seien $(n=110)$. Die Befragten, die diese Begründung wählten, sind mit der jetzigen Situation zufrieden und würden sich lieber weiterhin an muttersprachlichen Standards orientieren, selbst wenn sie diese nicht erreichen sollten (,Jeder erkennt das amerikanische Englisch. Wozu also noch mehr verschiedene Englischsprachen schaffen?!“ $[5 \mathrm{M}])$. Einige Studierende $(\mathrm{n}=29)$ wenden gegen Euro-Englisch ein, dass sich die Umsetzung des Konzeptes in der Praxis schwierig gestalten würde, ohne dafür Gründe anzugeben. Eine Untergruppe $(n=36)$ führt diesen Punkt jedoch weiter aus und thematisiert einen höheren Lernaufwand, der mit einer Einführung von Euro-Englisch aus ihrer Sicht einhergeht („Da durch eine gemeinsame Sprache jeder wieder auf etwas ,umgeschult" werden müsste“ [307L]). 
Wahrgenommene Eigenschaften von Euro-Englisch

In diesem Themenbereich stehen dem Euro-Englischen zugeschriebene Eigenschaften wie Künstlichkeit oder mangelnde sprachliche Reinheit im Vordergrund (vgl. Tab. 5).

Tabelle 5: Codes im Themenfeld „Wahrgenommene Eigenschaften von Euro-Englisch“

\begin{tabular}{|l|l|l|}
\hline \multicolumn{1}{|c|}{ Obercode/Untercode } & \multicolumn{2}{|l|}{ Anzahl/Gesamt } \\
\cline { 1 - 2 } Annahme, Euro-Englisch sei künstlich geschaffen & 85 & \multirow{2}{*}{145} \\
\cline { 1 - 2 } • $\quad$ Forderung, Euro-Englisch solle sich natürlich entwickeln & 27 & \\
\hline$\bullet \quad$ Annahme, Euro-Englisch sei schlechtes Imitat/unrein & 33 & \\
\hline
\end{tabular}

Insgesamt 145 mal wurden die Art der Entwicklung von Euro-Englisch sowie die damit verbundene Authentizität bzw. Künstlichkeit thematisiert. Allein der Obercode dieses Themenfeldes (,Annahme, Euro-Englisch sei künstlich geschaffen") ist mit 85 Nennungen einer der am häufigsten genannten Codes des Gesamtkorpus (z. B. „Man kann keine Sprache erfinden und diese den Bürgern aufzwängen" $[13 \mathrm{M}])$. Der Befund legt nahe, dass sich die Studierenden die Einführung von Euro-Englisch eher als konzertiertes top-down Vorhaben vorstellen. Eine weitere Deutung dieser Wahrnehmung könnte jedoch auch darin liegen, dass ein Großteil der Studierenden sich kein Bild davon machen konnte, wie sich ein europäisches Englisch im alltäglichen Gebrauch entwickeln würde. In jedem Fall legt dieses Ergebnis nahe, dass eine als künstlich wahrgenommene Sprache von vielen Studierenden nicht willkommen geheißen wird. Im ersten Untercode $(n=27)$ wird diese Idee aufgegriffen und gefordert, dass, wenn es schon ein Euro-Englisch geben müsse, dieses sich wenigstens 'natürlich' entwickeln sollte.

Davon etwas abgehoben ist die geäußerte Annahme, bei Euro-Englisch handle es sich um ein schlechtes Imitat einer 'richtigen', also als authentisch empfundenen Varietät des Englischen ( $\mathrm{n}=33$, „Das ist Unsinn und würde lediglich die engl. Sprache verschandeln“ [210G]). Das Urteil, dass Euro-Englisch lediglich eine minderwertige Kopie sei, kommt in negativ konnotierten Begriffen wie „Verfälschung“, „Mischmasch“, „,nachgemacht“, „verhunzt“, „,unrein“ oder „verschandelt" zum Ausdruck. Euro-Englisch ist dieser Wahrnehmung nach also keine für Europa akzeptable Mischform, in die sich die Herkunft ihrer Sprecher einbringen ließe, sondern eine 'zweitklassige' Kopie, deren Gebrauch nicht erstrebenswert erscheint. 


\section{Euro-Englisch und Kommunikation}

Dieses Themenfeld bündelt insgesamt sieben Codes, die sich hauptsächlich auf den von den Studierenden vermuteten kommunikativen Wert von Euro-Englisch beziehen (vgl. Tab. 6).

Tabelle 6: Codes im Themenfeld „Euro-Englisch und Kommunikation“

\begin{tabular}{|c|c|c|}
\hline Obercode/Untercode & \multicolumn{2}{|c|}{ Anzahl/Gesamt } \\
\hline Euro-Englisch erschwert Kommunikation (ohne weitere Begründung) & 41 & \multirow{4}{*}{88} \\
\hline - beschränkte Reichweite von EE: europäische versus globale Kommunikation & 33 & \\
\hline - $\quad$ EE erschwert Kommunikation mit Muttersprachlern & 8 & \\
\hline - EE verursacht Ausspracheprobleme & 6 & \\
\hline Euro-Englisch vereinfacht Kommunikation (ohne weitere Begründung) & 15 & \multirow{3}{*}{27} \\
\hline - EE könnte Eigenarten aller europäischen Sprachen berücksichtigen & 7 & \\
\hline - EE schafft gleiche/bessere Ausgangsbedingungen für alle & 5 & \\
\hline
\end{tabular}

Der Themenkomplex beinhaltet zwei Obercodes, die entweder eine kommunikative Beeinträchtigung $(\mathrm{n}=88)$ oder eine kommunikative Erleichterung durch Euro-Englisch $(n=27)$ beschreiben. Die 41 Nennungen, in denen nicht weiter begründet wird, warum Euro-Englisch die kommunikative Situation erschweren würde (z. B. „Ein Euro-Englisch verkompliziert den Sprachgebrauch“ [220A]), könnten dahingehend interpretiert werden, dass eine neue Form des Englischen von einigen Studierenden automatisch mit mehr Verwirrung und Problemen assoziiert wird. Neben diesem Obercode ist besonders ein Untercode hier von Interesse: 33 Studierende waren der Meinung, dass Euro-Englisch eine geringere Kommunikationsreichweite hätte, was wiederum mit der wahrgenommenen Aufgabe von Englisch als weltweitem Kommunikationsmedium konfligiere: „Im Zeitalter der Globalisierung wird nicht nur europaweit verhandelt, gesprochen, sondern weltweit" [5A]. Im Umkehrschluss hieße dies, dass diese Gruppe davon auszugehen scheint, dass ein Euro-Englisch nicht für Sprecher anderer Englishes verständlich wäre und somit ein auf den europäischen Kontext begrenztes Englisch sein müsste. Ähnliche Auffassungen spiegeln sich auch in den Codes „EE erschwert Kommunikation mit Muttersprachlern“ $(\mathrm{n}=8)$ sowie „EE verursacht Ausspracheprobleme“ $(\mathrm{n}=6)$.

Lediglich eine kleine Gruppe Studierender kann Euro-Englisch in kommunikativer Hinsicht etwas Positives abgewinnen $(\mathrm{n}=27) .15$ Antworten enthalten keine weiterführende Begründung; sieben weitere Studierende sehen einen kommunikativen Vorteil von Euro-Englisch darin, dass es Spezifika der Erstsprachen aufgreifen könnte („Besonderheiten europäischer Sprachen [könnten] dabei berücksichtigt werden" [73A]). Die in die Fachdiskussion eingebrachten Argumente für ein anpassungsfähiges und von Nichtmutter- 
sprachlern geprägtes Englisch scheinen somit nur von wenigen Studierenden geteilt zu werden.

\section{Abgrenzung und Gemeinsamkeit}

Dieses Themenfeld ist dem oben behandelten Komplex „Sprache und Identität“ ähnlich, bezieht sich jedoch speziell auf die abgrenzende bzw. Gemeinsamkeiten schaffende Funktion von Euro-Englisch (vgl. Tab. 7).

Tabelle 7: Codes im Themenfeld „Abgrenzung und Gemeinsamkeit“

\begin{tabular}{|l|c|c|}
\hline \multicolumn{1}{|c|}{ Obercode/Untercode } & \multicolumn{2}{|c|}{ Anzahl/Gesamt } \\
\cline { 1 - 2 } Identität über positiv empfundene Abgrenzung & 26 & \multirow{2}{*}{34} \\
\hline $\begin{array}{l}\text { Förderung von Identität, aber negativ empfundene Abgrenzung gegenüber } \\
\text { anderen }\end{array}$ & 8 & \multicolumn{2}{|c|}{30} \\
\hline Negativ empfundene Abgrenzung durch EE & \multicolumn{2}{|c|}{} \\
\hline
\end{tabular}

26 Studierende denken, dass Euro-Englisch einen separierenden Einfluss gegenüber anderen Englischvarietäten und deren Kulturen hätte und empfinden diese Trennung als identitätsstiftend und positiv (,ein ,Euro-Englisch“ würde sicherlich helfen, sich von den Briten/Amerikanern gedanklich ,abzugrenzen “" $[36 \mathrm{M}])$. Einige wenige Teilnehmer $(\mathrm{n}=8)$ geben allerdings zusätzlich zu bedenken, dass eine solche sprachliche Grenzziehung zu einer von ihnen als negativ empfundenen Isolation führen könnte. Von 30 Studierenden wird Euro-Englisch mit der Gefahr einer ausschließlich als negativ empfundenen Abgrenzung in Verbindung gebracht („Isoliert Europa von den anderen Staaten $\rightarrow$ könnte Aussenseiter werden“" [162L]).

\section{Alternative Forderungen}

Einige Befragte formulieren in ihren Freitextantworten Alternativen zu EuroEnglisch und dessen potenziell identitätsstiftender Wirkung (vgl. Tab. 8).

Tabelle 8: Codes im Themenfeld „Alternative Forderungen“

\begin{tabular}{|l|c|}
\hline \multicolumn{1}{|c|}{ Code } & Anzahl \\
\hline Neue Sprache entwerfen & 21 \\
\hline Eine einzige Sprache sollte als Weltsprache überall gelten & 20 \\
\hline Andere Sprachen für internationale/europäische Kommunikation & 13 \\
\hline
\end{tabular}

21 Studierende sehen in der Entwicklung einer komplett neuen Sprache (z. B. „Eurisch“ [17A]) eine Alternative zu Euro-Englisch. Diese Forderung gründet sich auf die Annahme, dass Euro-Englisch auf einem englischen Kern basiere, der nur durch eine Plansprache umgangen werden könne. 20 Studierende 
fordern, dass auf der ganzen Welt eine Weltsprache Gültigkeit haben sollte. Sie scheinen also die Auffassung zu vertreten, dass Variation für eine Sprache, die einen solchen globalen Status anstrebt, nicht wünschenswert sei. Nur sehr wenige Studierende $(n=13)$ möchten eine der vorhandenen Nationalsprachen außer Englisch, wie z. B. Deutsch, Französisch oder Spanisch, zur Kommunikation in Europa nutzen. Dieses Ergebnis könnte dahingehend gedeutet werden, dass sich die Mehrheit der Studierenden mit der Dominanz des Englischen abgefunden hat, auch wenn Deutsch mit 90 Millionen Sprechern die am meisten gesprochene Muttersprache in Europa ist.

\section{Wem gehört das Englische?}

Obwohl dieses Themenfeld zahlenmäßig mit 49 Zuordnungen weniger interessant erscheint (vgl. Tab. 9), ist es vor dem Hintergrund der Debatte um den 'Besitz' der englischen Sprache (z. B. Widdowson 1994) und der Orientierung an muttersprachlichen Standards für die Auswertung relevant.

Tabelle 9: Codes im Themenfeld „Wem gehört das Englische?““

\begin{tabular}{|l|l|c|}
\hline \multicolumn{1}{|c|}{ Obercode/Untercode } & \multicolumn{2}{|c|}{ Anzahl/Gesamt } \\
\hline Englisch gehört den Muttersprachlern & 36 & \multirow{2}{*}{49} \\
\hline$\bullet \quad$ Probleme für Muttersprachler des Englischen & 13 & \\
\hline
\end{tabular}

Die Ablehnung von Euro-Englisch mit der Begründung, Englisch gehöre seinen Muttersprachlern $(n=36)$, legt eine gewisse 'Verehrung' für die englische Sprache als Eigentum anderer nahe, aber auch die generelle Überlegung, dass man als Nicht-Muttersprachler kein Recht habe, in andere Sprachen einzugreifen und diese für eigene Zwecke zu normieren. Die Studierenden denken dabei offenbar vorwiegend an inner circle-Länder („Die englische Sprache ist korrekt, so wie sie in Großbritannien gesprochen wird. Ein Englisch, das davon abweicht, wäre also falsch und kein Englisch mehr" [211M]). In inhaltlicher Nähe zu diesem Code befindet sich die Annahme, dass Euro-Englisch zu Problemen für die Muttersprachler des Englischen führen würde $(n=13$, „Wenn es eine euro-englische Sprache gibt, in welchem Englisch sollen sich die Briten verständigen?" [19A]). In diesen Aussagen wird den Muttersprachlern also implizit zugestanden, dass sie die rechtmäßigen 'Eigentümer' des Englischen sind. Zusätzlich wird ein Perspektivwechsel vollzogen, bei dem antizipierte Probleme für Muttersprachler im Zusammenhang mit Euro-Englisch thematisiert werden. 
Die Auswertung abschließend und zur besseren Übersicht der Daten in den einzelnen Themenfeldern bietet Abbildung 1 (siehe unten) eine Auflistung der neun am häufigsten vergebenen Codes. Dazu wurden zusammengehörige Oberund Untercodes jeweils addiert und nur solche Zuordnungen berücksichtigt, die mindestens 40 mal vergeben wurden. Von den insgesamt 998 ausgewerteten Codes im Freitextkorpus sind 830 und dementsprechend ca. 83\% aller vorhanden Antworten in der Gesamtschau enthalten.

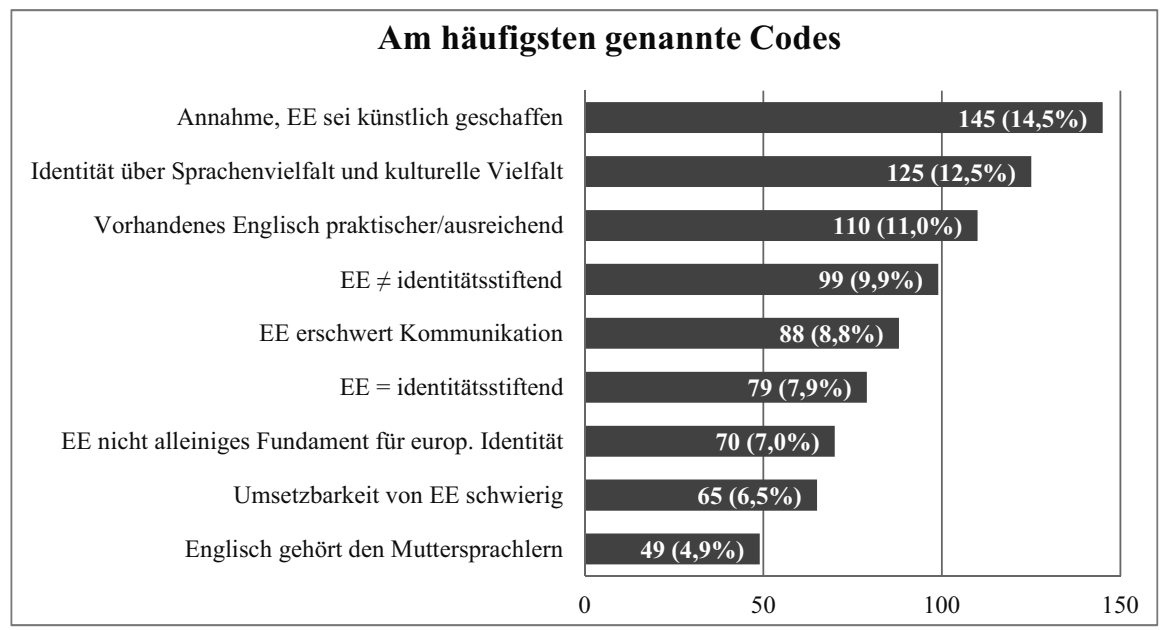

Abbildung 1: Die neun am häufigsten vergebenen Codes in der Auswertung der Freitexte

\section{$5 \quad$ Zusammenfassung und Ausblick}

Ausgehend von der Frage, ob Englisch als Verkehrssprache in Europa eine identitätsstiftende Wirkung entfalten kann, wurden zunächst verschiedene Identitätskonzepte im Hinblick auf den Zusammenhang zwischen Sprache und Identität diskutiert sowie sprachenpolitische Spannungsfelder der EU erörtert. Die Hauptergebnisse der darauf folgenden Freitextauswertung können wie folgt zusammengefasst werden:

- Die überwiegende Mehrheit der Befragten spricht sich dagegen aus, dass Euro-Englisch zu einer europäischen Identität beitragen kann. Viele Studierende ziehen eine 'natürliche' Entwicklung hin zu einer europäischen Form des Englischen durch die alltägliche Kommunikation nicht in Betracht und nehmen stattdessen an, dass ein Euro-Englisch planmäßig geschaffen werden müsse. Sie kritisieren in Folge dessen die als mangelhaft wahrgenommene Authentiziät einer solchen Form des Englischen: Eine als 'künstlich' bewertete Sprache wird von den Teilnehmern der Studie nicht als identitätsstiftend eingeschätzt. 
- Es herrscht eine starke Orientierung an muttersprachlichen Standards vor. Die Studierenden sehen überwiegend keine Notwendigkeit, eine eigene Form des Englischen $\mathrm{zu}$ sprechen und sind der Meinung, dass der Status quo ausreichend ist. Sie bringen zum Ausdruck, dass die Nutzung der bisherigen Varietäten des Englischen (vor allem britisches oder amerikanisches Englisch) sogar effizienter sei, auch weil sie annehmen, dass weitere Formen des Englischen nur zu mehr Verwirrung hinsichtlich der geltenden Standards führen würden. Einige Studierende sorgen sich sogar um die 'Eigentumsrechte' der Muttersprachler an der englischen Sprache und zeigen damit deutlich, dass sie die Möglichkeit, das Englische für ihre eigenen Zwecke zu modifizieren, für sich nicht in Anspruch nehmen wollen.

- Dass eine europäische Identität hingegen auf Mehrsprachigkeit basieren solle, wird ebenfalls häufig von den Studierenden angeführt. Viele dieser auf den ersten Blick für die EU-Sprachenpolitik sprechenden Aussagen lassen bei näherer Betrachtung jedoch ein anderes Verständnis von Mehrsprachigkeit erkennen: Die Studierenden meinen damit in den meisten Fällen die Koexistenz der Nationalsprachen innerhalb der EU, seltener jedoch beziehen sie sich auf die individuelle Mehrsprachigkeit.

Durch die Untersuchung konnte ermittelt werden, dass die Befragten in ihrer großen Mehrheit die Vorstellung ablehnen, dass eine eigenständige Lingua franca Englisch tauglich wäre, identitätsstiftend zu wirken. Ihre Identitätsentwürfe sehen die Studierenden in erster Linie von den Nationalsprachen bestimmt; es besteht offenbar nicht der Wunsch, die eigene sprachliche Identität über den Gebrauch einer europaspezifischen Variante des Englischen deutlich zu machen.

\section{Bibliographie}

Block, David (2007). Second Language Identities. London: Continuum.

Europäische Kommission (2007). Eurobarometer 66. Herbst 2006. Die öffentliche Meinung in der Europäischen Union. Online: http://ec.europa.eu/public_opinion/archives/eb/eb66/ eb66_de_nat.pdf (letzter Aufruf [26.03.2013]).

Europäische Kommission (2011). Eurobarometer 75. Frühjahr 2011. Die öffentliche Meinung in der Europäischen Union. Online: http://ec.europa.eu/public_opinion/archives/eb/ eb75/eb75_publ_de.pdf (letzter Aufruf [26.03.2013]).

Faez, Farahnaz (2011). Reconceptualizing the native/nonnative speaker dichotomy. Journal of Language, Identity and Education 10/4: 231-249.

Fiedler, Sabine (2011). English as a lingua franca - a native-culture-free code? Language of communication vs. language of identification. Apples - Journal of Applied Language Studies 5/3: 79-97. 
Gal, Susan (2011). Sociolinguistic regimes and the management of 'diversity'. In: Duchêne, Alexandre / Heller, Monica (Hrsg.): Language in Late Capitalism: Pride and Profit. London: Routledge. 22-42.

Gauck, Joachim (2013). Rede zu Perspektiven der europäischen Idee. Online: http://www.bundespraesident.de/SharedDocs/Reden/DE/Joachim-Gauck/Reden/2013/02/ 130222-Europa.html (letzter Aufruf [26.03.2013]).

Gnutzmann, Claus (2008). Can Euro English or English as a European lingua franca contribute to establishing a European identity? In: Rings, Guido / Ife, Anne (Hrsg.): NeoColonial Mentalities in Contemporary Europe? Language and Discourse in the Construction of Identities. Cambridge: Cambridge Scholars Publishing. 19-34.

Gnutzmann, Claus / Jakisch, Jenny / Koenders, Joana / Rabe, Frank (2012). Englisch als Verkehrssprache in Europa - identitätsstiftendes Medium für junge Europäer? Fremdsprachen Lehren und Lernen (FLuL) 41/2: 60-83.

House, Juliane (2006). Unity in diversity: English as a lingua franca for Europe. In: Leung, Constant / Jenkins, Jennifer (Hrsg.): Reconfiguring Europe: The Contribution of Applied Linguistics. London: Equinox. 87-103.

House, Juliane (2008). English as a lingua franca in Europe today. In: Extra, Guus / Gorter, Durk (Hrsg.): Multilingual Europe: Facts and Policies. Berlin: de Gruyter. 63-83.

James, Allan (2008). The challenges of the Lingua franca: English in the world and types of variety. In: Gnutzmann, Claus / Intemann, Frauke (Hrsg.): The Globalisation of English and the English Language Classroom. 2. Aufl. Tübingen: Narr. 133-144.

Jenkins, Jennifer (2007). English as a Lingua Franca: Attitude and Identity. Oxford: Oxford University Press.

Kachru, Braj (1985). Standards, codifications and sociolinguistic realism: The English language in the outer circle. In: Quirk, Randolph / Greenbaum, Sidney (Hrsg.): English in the World: Teaching and Learning the Language and Literatures. Cambridge: Cambridge University Press. 11-30.

Kuckartz, Udo (2010). Einführung in die computergestützte Analyse qualitativer Daten. 3. Aufl. Wiesbaden: VS Verlag für Sozialwissenschaften.

Küster, Lutz (2007). Multiliteralität und Europa-Identität: Zielsetzungen und Realisierungsperspektiven vor dem Hintergrund europäischer Sprach- und Bildungspolitik. In: Elsner, Daniela / Küster, Lutz / Viebrock, Britta (Hrsg.): Fremdsprachenkompetenzen für ein wachsendes Europa: Das Leitziel „,Multiliteralität“. Frankfurt/M.: Lang. 35-48.

Modiano, Marko (2001). A new variety of English. English Today 17/4: 13-14.

Modiano, Marko (2003). Euro-English: A Swedish perspective. English Today 19/2: 35-41.

Mollin, Sandra (2006). Euro-English: Assessing Variety Status. Tübingen: Narr.

Mundschau, Heinz (1995). Europa 2000. Praxis des Neusprachlichen Unterrichts 42/4: 339345.

Nissen, Sylke (2004). Europäische Identität und die Zukunft Europas. Aus Politik und Zeitgeschichte 38: 21-29.

Norton, Bonny (1997). Language, identity, and the ownership of English. TESOL Quarterly 31/3: 409-429. 
Norton, Bonny / Toohey, Kelleen (2011). Identity, language learning, and social change. Language Teaching 4/4: 412-446.

Pölzl, Ulrike (2003). Signalling cultural identity: the use of L1/Ln in ELF. Vienna English Working Papers 12/2: 3-23.

Seidlhofer, Barbara (2005). English as a lingua franca. In: Hornby, Albert S. (Hrsg.). Oxford Advanced Learner's Dictionary of Current English. Oxford: Oxford University Press. Reference section 92 (s.p.).

Seidlhofer, Barbara (2011a). Understanding English as a Lingua Franca. Oxford: Oxford University Press.

Seidlhofer, Barbara (2011b). Conceptualizing 'English' for a multilingual Europe. In: De Houwer, Annick / Wilton, Antje (Hrsg.): English in Europe Today: Sociocultural and Educational Perspectives. Amsterdam: Benjamins. 133-146.

Tabouret-Keller, Andrée (1997). Language and identity. In: Coulmas, Florian (Hrsg.): The Handbook of Sociolinguistics. Oxford: Blackwell. 315-326.

Widdowson, Henry G. (1994). The ownership of English. TESOL Quarterly 28/2: 377-389. 
II.

Multiliteracies and the Construction of Plurilingual Identities in Foreign Language Learning and Teaching 



\title{
'Bildung', 'Multiliteracies' and 'Identity' - Key Concepts in Language Education in the Light of Sociocultural Theory and Dynamic Systems Theory
}

Stephan Breidbach / Lutz Küster (Berlin)

\begin{abstract}
Theoretische und empirische Studien der jüngeren Fremdsprachenforschung haben den Blick geschärft für die Pluralität der Erscheinungsformen und für die Komplexität der Bedingungsfaktoren sowohl von Sprachgebrauch als auch von Sprachenlernen. Dabei hat die Rezeption von Ansätzen der Sociocultural Theory zu einem verstärkten Bewusstsein für die soziale und interaktive Kontexteinbettung von Sprachlernen geführt. Pluralität und Komplexität sind daher aus gutem Grund konstitutive Merkmale der in fremdsprachendidaktischen Diskursen diskutierten Schlüsselbegriffe 'Bildung', 'Identität' und 'Multiliteralität'. Insbesondere Komplexität und Emergenz bilden darüber hinaus Leitaspekte der vorwiegend im englischsprachigen Raum verbreiteten Dynamic Systems Theory. Der vorliegende Beitrag geht den wechselseitigen Bezügen der mit den genannten Konzepten verbundenen fremdsprachendidaktischen Forschung nach und versucht hiervon ausgehend, Perspektiven für die theoretische Rahmung reflexiven Sprachenlernens aufzuzeigen.

Deux courants actuels de la recherche en Linguistique Appliquée marquent le cadre de référence du présent article: les théories socioculturelle et émergentiste (cette dernière étant connue dans l'espace anglophone sous le nom de Dynamic Systems Theory). L'une comme l'autre mettent l'accent sur l'importance du contexte en tout apprentissage. En cela, elles révèlent un certain nombre de parallèles avec les concepts mis en avant dans le titre de cet article. L'objet de celui-ci est de dresser un bilan des liens qui peuvent être établis entre les différents discours mentionnés et qui pourraient - telle est sa conclusion pour l'instant très provisoire - servir de cadre théorique du concept d'apprentissage réflexif.
\end{abstract}

\section{Introduction ${ }^{1}$}

'Plurilingualism' and 'multiliteracies' - the two central concepts of this volume - have common ground in that they reflect the wide-ranging transformations of public, private and professional life in the age of globalisation. In the academic discourse, we find them used both in a descriptive and in a normative way. In descriptive terms, plurilingualism is considered a reality not only in traditionally multilingual areas, but also a result of growing linguistic and cultural heterogeneity in societies with strong migrational influences. Likewise, the term

\footnotetext{
${ }^{1}$ We would like to thank Claudia Schmidt and José Medina for their helpful comments on a previous version of this article. However, we remain fully responsible for any inconsistencies and errors.
} 
'multiliteracies' comprises communicative practices we use daily in dealing with multimodal information, for example on the internet. In normative terms, both concepts describe educational aims: In order to cope with the plurality and complexity of communicative codes and situations, young people will need qualifications different from those taught to former generations. Hence, concepts of language education will need to look beyond teaching single languages individually, and a much broader perspective will be required on multilingual education, integrated into a wide-ranging system of "language across the curriculum'. Such concepts will have to recast the traditional dichotomy between the oral and the written while also taking into account the variety and hybridity of texts and communication.

In this chapter, we will look at some key concepts we consider to be elements of a pedagogical framework, which could equally inform classroom practices for plurilingualism and multiliteracies and empirical research in the field of second/further language education. However, our argument will be tentative and preliminary in the way that we will try to assess the potential offered by Sociocultural Theory (SCT) and Dynamic Systems Theory (DST) for theorising notions such as Bildung, multiliteracies and identity, all of which have some currency in language education at the moment. Our aim is neither to provide an in-depth analysis of each theoretical discourse and concept we will refer to in the following, nor a pedagogical blueprint, but rather to point out directions for further foundational work and future empirical research.

In the following, our argument will be developed in eight sections. After a short prelude, in which we will reflect on the significance of context in language education and Second Language Acquisition (SLA) research, we will continue with two brief and arguably selective readings of SCT and DST approaches. In the following three sections, we will put the focus subsequently on the concepts of Bildung, multiliteracies and identity. We will then propose elements taken from these concepts as analytical tools in an exemplary discussion of narrative data from a language learner, and finally highlight some issues for further research.

\section{Prelude: The significance of 'context'}

To start with, we would like to address the significance of 'context' which has become a central concern in Second Language Acquisition (SLA) research. Taking a sociocultural perspective, we consider learning not as a purely mental and individual activity, but also as a social one, based on interpersonal interaction. Language in the words of Bonny Norton "is not conceived of as a neutral medium of communication, but is understood with reference to its social meaning" (Norton 2000: 5). Starting from here, language use and language 
learning are in our understanding closely linked to processes of negotiation of meaning, but also to meaning-making in a broader, Humboldtian sense: Language is not just a means of communicating pre-existing ideas; it is to no lesser degree a means of constructing knowledge in search for personal orientation. In Wilhelm von Humboldt's terminology, this search is a process of Bildung, which mainly consists in clarifying one's own relationship to the world and to the self (Selbst- und Weltverhältnis). Hence, language learning can, of course, be considered in a technical sense as the expansion of available communication tools, but also in a more holistic way as the transformation of one's concept of self and one's position in and towards the world.

When we focus on Bildung, multiliteracies or identity and extend our perspective by discussing these concepts $v i s-\grave{a}-v i s$ some of the main tenets of Sociocultural Theory (SCT) and Dynamic Systems Theories (DST), we will do so for the following reasons. As far as we can see, SLA-studies based on DST mainly take into view the mental processes of language acquisition, whereas in SCT a perspective on the social framing of language acquisition and use prevails. Still, for both theoretical approaches, dealing with what in SCT terminology is 'context' (in DST terms: complexity) is a major concern. In DST, complexity refers to the supposition that language acquisition relies on a multitude of interrelating factors, whose interaction can neither be fully described nor can the outcome of such interaction precisely be predicted. In SCT, context refers to the (Vygotskian) notion that humans are culturally "mediated beings" (Lantolf 2006: 69). While contemporary conceptualisations of Bildung or multiliteracies explicitly draw on SCT, emergentist approaches can be found in recent studies on motivation, learner autonomy and identity (cf. Dörnyei 2010; Murray et al. 2011). Against this common division, we assume that theorising Bildung, multiliteracies and identity from both SCT and DST perspectives may indeed be fruitful.

\section{A brief look at socioculturalist approaches in SLA-research}

Vygotsky's understanding of language acquisition as an internalization of social interaction may be considered the baseline of all sociocultural approaches. Learning a language relates to communication with others and to acting by speaking in specific communicative situations, while the language is gradually embodied by the learner (cf. Kramsch 2000; Küster 2012; Lantolf/Thorne 2006). Based on the activity theory put forth by Vygotsky and Leontiev, it was assumed that mental development cannot evolve as separated from active interaction with one's material and symbolic environment, but rather emerges from this 
negotiation. ${ }^{2}$ At the same time, an evolving self guides one's own acting by providing personal aims. Accordingly, theoretical interdependencies between identity constructions and learner motivation on the one hand, and the course of communicative interaction and language learning on the other hand, can be found (cf. Lantolf/Thorne 2006: 209-262; Pavlenko/Lantolf 2000: 171-174).

The concept of 'communities of practice' introduced by Lave/Wenger (1991) and Wenger (1998) is based on the general idea that all learning is to be understood within the context of interpersonal experiences. What proved to be a leading concept in particular is that of 'legitimate peripheral participation': Learning, and especially second language learning, is seen as a process of individual experience, in which the learner is gradually involved and integrated in the speech community. Starting as a marginally integrated novice s/he, by increasingly participating in social interaction, expands her/his competences. In the wake of Lave and Wenger's work, specific learning contexts, mainly those related to non-directed language acquisition, came to the fore in research (cf. Block 2007: 865; Norton 2001). Positioning theory, for example, made it possible to understand the contingent nature of identity constructions made by individuals through their own active positioning within communicative situations (cf. Norton/Toohey 2011: 418). In terms of context dependency, connections can be drawn to the concept of 'situated identities' (cf. Ushioda 2011: 205).

Following Bourdieu, the aspect of the "relations of symbolic power" (Bourdieu 1977: 646), which becomes manifest in the question of how the individual gains access to second language communities and, if necessary, responds to resistance, is emphasized by Norton. She reports on case studies which show in many facets how adult learners struggle not to be marginalized, humiliated, embarrassed or even excluded in contexts of language learning and verbal communication (Norton 2000, 2001). Finally, Bourdieu's notion of cultural and social capital lays the foundation for Norton's idea that intentionally learning a second/foreign language can be conceptualized as investment (investment theory).

A conjunction between sociocultural, poststructuralist and humanistic tendencies is to be found in the wide-spread Language Socialization (cf. Duff/ Talmy 2011) and Language Ecology approaches. Kramsch/Wellmon (2008), for example, combine Language Ecology, German theories of Bildung and Kramsch's concept of Symbolic Competence in a systematic way. They under-

\footnotetext{
${ }^{2}$ Larsen-Freeman/Cameron (2008: 157, 160, note 16) note that much of the conceptual compatibility of DST and Vygotsky-related SCT approaches to language learning depends on whether the relation between mental and external social activity is thought of as dialectic or dialogic. Accordingly, only the latter view would grant commonalities with DST.
} 
line that 'Language Ecology' is far from being founded on a coherent theory of language, its core element being rather "a holistic way of viewing language in its social and cultural environment" (ibid.: 219; cf. also Kramsch 2008; Kramsch/Whiteside 2008). In contrast to other studies informed by sociocultural thought, this approach explicitly refers to Complexity Theory.

\section{Some elements of emergentist approaches in SLA-research}

Emergentism has its origins in natural sciences, namely in the science of chaos/complexity. Chaos Theory and its derivatives Complexity Theory and Dynamic Systems Theory (or Dynamic Adaptive Systems Theory) were developed to understand processes which cannot be explained within a logic of linear causality, for example in disciplines such as meteorology and astronomy. 'Emergence' as a central concept of these approaches describes the phenomenon of qualitative change in a developmental process. But contrary to linear causality, which explains changes as direct consequences of preceding states or interceptions, emergence is not predictable in time or nature. The reason for this is that it depends on the interactions of different parts within a complex system, and neither the exact result of such interactions nor their temporal dimension can be fully predicted. A popular example is the so-called butterfly effect, i.e. "the notion that a butterfly fluttering its wings in a distant part of the world today can transform the local weather pattern next month" (Larsen-Freeman 1997: 144). However, the course of such events is not characterized by randomness. Emergentist studies, in fact, reveal that processes within complex systems tend to give rise to moments of stability called 'attractor states'. These are patterns characterized by a fractal structure, fractals being phenomena that show characteristics of self-similarity at different stages.

Diane Larsen-Freeman (1997) first introduced elements of chaos/complexity theory into SLA-research. Meanwhile, this approach has gained some ground in the discipline (e.g. de Bot et al. 2007; Mercer 2001; van Geert 2008), and Larsen-Freeman herself continued investigating in this direction (cf. e.g. Ellis/Larsen-Freeman 2006; Larsen-Freeman 2011; Larsen-Freeman/Cameron 2008). What attracted researchers to DST was the perception that previous language acquisition theories had not been able to explain the interaction of complex factors. Variables that are thought to have an impact on language acquisition could not be controlled empirically to a sufficiently high degree to put researcher in a position to project and predict outcomes in detail. While DST claims this unpredictability to be inevitable, it still offers implications for the learning/teaching practice. According to DST-based learning theory (e.g. Emergentism), a complex learning environment is necessary to facilitate emergence. This clearly parallels the main ideas related to a rich learning context in 
constructivism. Further consequences pertain to the importance of the initial phases of a learning process and to the use of resources.

\section{$5 \quad$ Bildung, Sociocultural Theory and Emergentism}

The notion of Bildung originates in the era of late Rationalism and early Romanticism of the late $17^{\text {th }}$ and early $18^{\text {th }}$ centuries. Bildung describes the individuals' becoming an identifiable subject through a process of increasingly complex and diverse ways of relating to the world. Wilhelm von Humboldt conceived of the process of Bildung as an on-going interaction between the individual and the experiential world. In the course of this process, the individual draws on a distinctly human resource, which Humboldt calls Kraft (resp. Kräfte), by virtue of which people generate a meaningful concept of themselves and the world (cf. also Wimmer 2003: 185, note 1). While Humboldt thought of Bildung as a reconciled and ultimately as a balanced totality of each of these Kräfte, more recent reformulations of the notion of Bildung draw on postmodern epistemology, leading to a stronger emphasis of divergence - as opposed to classical convergence - of such modes of accessing the world. Contemporary models of Bildung see the individual engaged in negotiating rather than reconciling diverse modes of relating to the world.

For our context, the theoretical and empirical work done by Hans-Christoph Koller (2012) offers an interesting perspective. Koller's understanding of Bildung is based on a differentiation of 'learning' and Bildung. While learning denotes any acquisition of information or knowledge within an existing schema, processes of Bildung are to be thought of as a particular kind of "higher-order learning" (ibid.: 15, our translation), through which the "fundamental figurations of how a person relates self and world change" (ibid., our translation). ${ }^{3}$ Drawing on the work by Rainer Kokemohr (2007), Koller continues to argue that changes of this kind may be triggered in situations in which a person fails to assimilate new knowledge according to his/her existing mental schemas, and simultaneously experiences this failure as a cognitive, emotional or any other sort of crisis.

Following from this, Koller (2012: 16ff.) raises four major issues which he believes a contemporary theory of Bildung should cover:

- How can the structure of 'fundamental figurations of self and world' appropriately be theorised?

\footnotetext{
${ }^{3}$ Koller's own summary account of what constitutes processes of Bildung reads: "Bildungsprozesse bestehen demzufolge also darin, dass Menschen in der Auseinandersetzung mit neuen Problemlagen neue Dispositionen der Wahrnehmung, Deutung und Bearbeitung von Problemen hervorbringen, die es ihnen erlauben, diesen Problemen besser als bisher gerecht zu werden.” (Koller 2012: 16)
} 
- How can the structure of such instances of crisis (Problemlagen) be theorised that (potentially) initiate processes of Bildung?

- How can such processes of higher-order learning be conceptualised to explain the emergence of new fundamental figurations?

- What are the appropriate methodological instruments to access processes of Bildung empirically?

We cannot present Koller's account of the concept of Bildung here in full. However, his understanding of Bildung seems to share a number of conceptual characteristics with aspects of Sociocultural and Dynamic Systems Theory.

For the moment, we leave aside the first and second question from the list above and turn to the third. Koller (ibid.: 112ff.) points out that the subject may neither be aware of nor be in control of higher-order learning transformations at the moment of their occurrence. Koller refers to Ulrich Oevermann (1991), who speaks of the 'emergence of the new' and argues that the outcome of a higherorder learning process, on top of being invisible and undecipherable to a person while she/he is undergoing this process, is not predictable. Only retrospectively do new figurations become recognisable. However, it remains an open question whether 'Bildung' should be distinguished from 'identity' by the metacognitive ability to reflect on one's "relationship to the world" (Norton 2000: 5). If we do so, referring to 'Bildung' would necessarily imply an element of metacognition, whereas referring to 'identity' would not (while at the same time not excluding it).

Taking a final step before turning to the concept of multiliteracies, we would like to raise a point for further discussion, again without being able to provide answers here and now: If the process of Bildung can be described as an emergent phenomenon, can other key concepts of Dynamic Systems Theory also be brought into play for theorising Bildung? Will it, consequently, be feasible to apply DST in research of processes of higher-order learning within the context of second or foreign language learning? If we follow Humboldt's notion that Bildung occurs in and through the individual's interaction with his/her material and (social) world, processes of Bildung become fundamentally dependent on context. Context is therefore not to be excluded from the picture as 'noise'. In the same vein, de Bot et al. (2007) argue that DST allows to "merge the social and cognitive aspects of SLA and show how their interaction can lead to development" (ibid.: 19).

It would be tempting to explore other features of higher-order learning through the lens of DST as dynamic, recursive and reflexive. It would be equally tempting to explore the tools of empirical research used in both contexts. For reasons of limited space, we will do neither and turn to the concept of multi- 
literacies for a similar investigation of underlying links with the theory of Bildung, DST and Emergentism.

\section{Multiliteracies, Sociocultural Theory and Emergentism}

Having been developed in the late 1990 s, the concept of multiliteracies is meant to provide pedagogical answers to questions which derive directly from the social changes in western societies and from the changes in media-based communication. The demands seen by a group of researchers and practitioners, named The New London Group, relate to preparing young learners for a life in contexts of multilingual, multicultural and, in a Bakhtian sense, multivocal communication, digital communication, global and local participation and a complex knowledge society. The main idea is that traditional linear literacy focusing on the alphabet of one single language is no longer suited to cope with these demands. Future education should in fact take into account the increasing linguistic and cultural diversity in the classroom, foster children's language competencies in both schooling and heritage languages and introduce them to visual and computer literacies. According to the idea of education for citizenship, learners should also be enabled to develop critical literacy and social awareness rather than mere instrumental skills. This is basically what the New London Group in the subtitle of their manifesto refers to as the "design of social futures".

The didactic and methodological outlines proposed by the New London Group are marked by a sociocultural approach taking a holistic view of the human: "Our view of mind, society and learning is based on the assumption that the human mind is embodied, situated and social" (The New London Group 1996: 18). In this perspective, learners' social identity and social participation are points of major interest. In brief, the concept of multiliteracies (or 'multimodal literacies') can be defined as the capacity of learners to negotiate and generate (new) meaning in linguistically and culturally heterogeneous lifeworlds, using 'old' and 'new' media and adopting responsibility for themselves as well as for the community. This capacity requires above all the ability to combine and to integrate heterogeneous, complex elements in learning, thinking and acting.

If we ask about the relationship between the concept of multiliteracies and sociocultural theory, the answer is quite obvious as the writings of the New London Group explicitly refer to Vygotskian ideas. Learning is seen as a social process, or more precisely as a constantly modified result of social interaction, which concerns the individual as a whole not only in contexts of formal learning but in contexts of private, professional and political life as well. To our knowledge, no research has yet been done concerning emergence in multiliteracy 
acquisition or practices. In view of the looming complexity, a DST-informed mindset might best be suited for an analysis of these processes.

As we have seen, the concepts of multiliteracies and Bildung both depart from a holistic view of the individual and emphasize his/her interaction with environmental factors particularly within sociocultural contexts. Whereas theorizing the notion of Bildung has also given rise to qualitative empirical investigations concerning the identity implications of Bildung (mainly through biographical narrative inquiry and reconstructive designs in the field of Bildungsgangdidaktik), comparable research has yet to be tackled for an understanding of the development of multiliteracies.

\section{Identity, Sociocultural Theory and Emergentism}

The concept of identity has been an issue of controversial debate for quite a long time. Early sociological studies thought of identity as an essentially static construct of affiliating to social groups (cf. Ricento 2005: 897). At present, however, identity is seen as an individual and, after all, never-ending process of selfrelating in changing social contexts. Thus, individual identity constructions are always dynamic and at the same time multi-faceted, as reflected in the concepts of fluid, situated and multiple identities. Bonny Norton, for example, underlines its dynamic and temporary character by defining 'identity' as "how a person understands his or her relationship to the world, how that relationship is constructed across time and space, and how that person understands possibilities for the future" (Norton 2005: 5).

Learning-/learner-oriented identity research was formed not only by sociocultural but also by post-structuralist influences (cf. Norton/McKinney 2011; Norton/Toohey 2002). Pointing to the sensitive issue of power relationships in language practice, Norton/Toohey (2011: 415) see language learning "as a process of struggling to use language in order to participate in specific speech communities". Additionally, poststructuralist thought is reflected in the way subjectivity and language are considered as inextricably intertwined (cf. ibid.: 417): The individual is subject to language as well as its creative user. Based on these principles, Bonny Norton (2000: 127f.) developed a theory of identity as it is encapsulated in the frequently quoted notion of identity as a field of controversy and as a "complex site of struggle", where people fight for recognition (cf. also Block 2007: 866f.; Dörnyei/Ushioda 2009b: 4f.). Empirical findings suggest that learning a second/foreign language interferes in the interdependencies of self- and world-perception. Learning and processes of selfpositioning are hence seen as irresolvably interwoven.

Sociocultural identity research carries rich potential due to its contiguity to language learning approaches based on theories of Bildung, especially within the 
so called Bildungsgangdidaktik (cf. Decke-Cornill/Hu/Meyer 2007; Meyer/ Reinartz 1998). This line of research particularly focuses on long-term manifestations of learning within learners' overall personalities. It thus draws attention to the personal relevance of foreign language learning and to its developmental perspective over longer periods of time. However, it remains a matter of debate whether sociocultural perspectives allow for a sufficient explanation of mental learning processes. In this respect, approaches in learning theory may usefully be based on Complexity Theory and its derivatives, DST and Emergentism. A link between these two is also suggested in studies on identity constructions of foreign language learners, which increasingly consider both theoretical approaches to be mutually compatible. Ushioda (2011) argues that language learning motivation can be conceptualised as a function of identity. In fact, complexity as a metaphor and the theory of Complex Adaptive Systems as a new paradigm can provide important stimuli for identity research. Sade (2011: 45), for example, puts the focus on the concept of attractors and the fractal dimension as tools for understanding the dynamics of identity constructions. In this perspective, empirical research may particularly benefit from longitudinal designs.

As case studies by Sade, Lamb, Paiva and Oxford (all 2011) and others demonstrate, the theoretical background of current identity research proves prolific in the context of second/foreign language learning. As an example, the dimension of collective identities has been conceptualized using DST inventory, above all by the notion of attractors (cf. Sade 2011: 46). All in all, complexity approaches have gained influence in motivation research related to language learning (cf. Dörnyei/Ushioda 2009c; Dörnyei 2010; Ushioda 2009). On the whole, however, research in this particular field is still at its outset. Taking a slightly different direction, we now briefly turn to phenomena of reflective learning.

\section{Reflective learning and foreign language education}

DST stipulates emergence (Larsen-Freeman/Cameron 2008: 58ff.) in learning processes. Being associated with higher-order learning, processes of Bildung probably follow this pattern as well. As we suggested with Koller (2012), for a change in the 'fundamental figurations' of one's relations to the world and self to accur, a situation of crisis, in which formerly held schemas become dysfunctional, may be required as a necessary pre-condition. The imminent transformation may build up over time resulting in an ad-hoc change, like a breaking wave, or it may come unnoticed through continuous smaller changes. Sarah Mercer (2011) describes agency as a complex dynamic system. She shows how in the case of a student teacher of English and Italian, learning experiences and a changing sense of linguistic competence function as contexts for the 
development of a sense of agency, leading to a growth in professional identity. The student teacher in question moves beyond a restricted professional identity to a more expansive way of relating to her professional self, and to language teaching as her future professional field.

For an investigation of reflective learning in languages, the context of preservice language teachers is particularly interesting. In order to establish a professional identity, student teachers need to negotiate 'identity options' within at least three dimensions more or less simultaneously: language learner, user and prospective teacher (Chik/Breidbach 2011: 554). Reconstructing 'identity options' and tracing their professional development may lead to a deeper understanding of how reflective language learning works.

In the following excerpt, Tess ${ }^{4}$, who at the time of writing was a student teacher at a German university, tells her story of her year abroad. We use this part of her written language learning history to illustrate what we recognise as the emergence of an integrated understanding of language learning.

[...] Becoming part of South African society, I wanted to be able to express myself among others as appropriately as possible. I figured out that English provides expressions with certain connotations that don't have an exact substitute in German and vice versa. While I experienced the differences in languages I became more conscious of a meta-level of language and realized that one doesn't only carry facts by words, but that the words I choose to express a subject suggest how I feel about it.

This led me to a simple conclusion: The more words I have stored in my mind, the more likely it is that I find the words that best suit my thoughts and feelings. [...] So I listened and talked, went out to many cultural occasions, took part in church services, cooked with Indian women, went to dances in the temple, worked in children's homes, travelled and every experience became a language experience as well. [...]

Starting from the desire to interact appropriately in a new language and social context, Tess finds that English expands her possibilities of expression; this leads her to reflect on the communicative capacities of language. To be able to use this capacity in full, she sets herself a learning aim, which is to learn as many words as she can 'store' in her memory. For this, she seeks participation in social and cultural events, and she even finds a workplace. For Tess, in this process of becoming aware of her own learning leads to the next. Eventually, she is able to experience her being part of "South African society" as a language experience - and vice versa. The structure and wording of her narrative suggests that at some stage in the process, a qualitative change has occurred from a rather linear and accumulative to a dynamic and arguably more complex and holistic understanding of language learning.

\footnotetext{
${ }^{4}$ The real name of the student has been changed.
} 
The text also shows that Tess negotiates her 'identity options' as a language learner and language user. As a language user, she aims to become a part of South African Society, which is concomitant for her with a need to express herself affectively and emotionally. As a language learner, her efforts to overcome the social distance affect her way of thinking about language. This is what seems to happen when she becomes aware of the capacities of language for allowing herself to adopt a new voice. The last sentence suggests that a new figuration has emerged when Tess writes of herself in terms of learner as user, who experiences language learning through active involvement in social events. In what particular way this new idea about language learning and using will affect her developing teacher identity does not seem to be a part of her explicit narrative. From a DST perspective, however, in which all of Tess's identity positions can be seen as interdependently related, we may assume that in the long run, some effect will be made. However, when this will happen, in which way and with what outcome, is beyond prediction.

\section{Research perspectives}

Since the publication of Larsen-Freeman's seminal article in 1997, DST has gained ground in Applied Linguistics and SLA research beyond a purely metaphorical use. Within the German context, Bleyhl even as early as 1989 (cf. Bleyhl 1989: 33f.) draws on concepts such as self-organisation and metaphorises language learning as a 'meandering river', suggesting an 'ecologic' understanding of language didactics ("ökologische Didaktik", ibid.: 34). The development of language within and outside classroom contexts has attracted much interest of researchers in the meantime. What seems to be missing is wide-ranging research on the development of the language learner. Also, much of what is discussed as 'intercultural learning' builds on the assumption that language learning affords opportunities for reflective learning. However, a satisfactory conceptual (as opposed to a mainly metaphorical) understanding of how this should come about in actual teaching, has not yet been developed.

Both such and similar research lead us to the general question whether language learning can function as an ecological parameter for Bildung in the sense of leading to a change in the 'fundamental figurations' of how a person relates to the world and himself/herself.

- Are there existing threshold levels for linguistic skills to become critical for the perception of learners' sense of self/identity?

- Are such thresholds dependent on other contextual parameters? And in particular: In which way does the quality of learning experiences (or the perception of these) form an ecologic variable for processes of Bildung to emerge? 
- To what extent does second/further language learning interact with acquisition processes of multiliteracies?

- What potential impact do incidents of reflective learning ('becoming aware') have on system dynamics in learners? How do such incidents interact with internal and contextual parameters?

We know remarkably little about how - albeit plurilingual - language learning interacts with the acquisition of multiliteracies. We know little about processes of Bildung in a broad sense in conjunction with language learning. We believe, however, that a combined SCT and DST approach can help to close this gap.

\section{References}

Atkinson, Dwight (2011) (ed.). Alternative Approaches to Second Language Acquisition. Abingdon: Routledge.

Bleyhl, Werner (1989). Psycholinguistische und pragmatische Überlegungen zum handlungsorientierten Fremdsprachenunterricht. In: Bach, Gerhard / Timm, Johannes-Peter (eds.): Englischunterricht. Tübingen: Francke. 22-41.

Block, David (2007). The Rise of Identity in SLA Research, post Firth and Wagner (1997). The Modern Language Journal 91: 863-876.

Bourdieu, Pierre (1977). The economics of linguistic exchange. Social Sciences Information 16/6: 645-668.

Chik, Alice / Breidbach, Stephan (2011). Online Language Learning Histories Exchange: Hong Kong and German Perspectives. TESOL-Quarterly 54/3: 553-564.

de Bot, Kees / Lowie, Wander / Verspoor, Marjolijn (2007). A Dynamic Systems Theory aproach to second language acquisition. Bilingualism: Language and Cognition 10/1: 7-21.

Decke-Cornill, Helene / Hu, Adelheid / Meyer, Meinert A. (eds.) (2007). Sprachen lernen und lehren. Die Perspektive der Bildungsgangforschung. Opladen / Farmington Hills: Budrich.

Dörnyei, Zoltan (2010). The relationship between language aptitude and language learning motivation: individual differences from a dynamic systems perspective. In: Macaro, Ernesto (ed.): Continuum Companion to Second Language Acquisition. London: continuum. 247-267.

Dörnyei, Zoltan / Ushioda, Ema (eds.) (2009a). Motivation, Language Identity and the L2 Self. Bristol: Multilingual Matters.

Dörnyei, Zoltan / Ushioda, Ema (2009b). Motivation, language identities and the L2 Self: A theortical overview. In: Dörnyei/Ushioda (2009a): 1-7.

Dörnyei, Zoltan / Ushioda, Ema (2009c). Motivation, language identities and the L2 self: Future research directions. In: Dörnyei/Ushioda (2009a): 350-356.

Duff, Patricia A. / Talmy, Steven (2011). Language socialization approaches to Second Language Acquisition. Social, cultural, and linguistic development in additional languages. In: Atkinson, Dwight (ed.): 95-116. 
Ellis, Nick C. / Larsen-Freeman, Diane (2006). Language emergence: implications for applied linguistics: Introduction to the special issue. Applied Linguistics 27/4: 558-589.

Kokemohr, Rainer (2007): Bildung als Welt- und Selbstentwurf im Fremden. Annäherungen an eine Bildungsprozesstheorie. In: Koller, Hans-Christoph / Marotzki, Winfried / Sanders, Olaf (eds.): Bildungsprozesse und Fremdheitserfahrung. Beiträge zu einer Theorie transformatorischer Bildungsprozesse. Bielefeld: transcript. 13-69.

Koller, Hans-Christoph (2012). Bildung anders denken. Einführung in die Theorie transformatorischer Bildungsprozesse. Stuttgart: Kohlhammer.

Kramsch, Claire (2000). Social discursive constructions of self in L2 learning. In: Lantolf, James P. (ed.): Sociocultural theory and second language learning. Oxford: Oxford University Press. 133-153.

Kramsch, Claire (2008). Ecological perspectives on foreign language education. Language Teaching 41/3: 389-408.

Kramsch, Claire / Wellmon, Michael Chad (2008). From Bildung durch Sprache to Language Ecology: The uses of symbolic competence. In: Doff, Sabine / Hüllen, Werner / Klippel, Friederike (eds.): Visions of Languages in Education. Berlin: Langenscheidt. 215-225.

Kramsch, Claire / Whiteside, Anne (2008). Language Ecology in multilingual settings. Towards a theory of Symbolic Competence. Applied Linguistics 29/4: 645-671.

Küster, Lutz (2012). Vernetzendes Lernen: Eckpunkte eines Forschungsvorhabens zu Zielen und Praktiken der Multiliteralität in ihrer Relevanz für Lerneridentitäten. In: Leitzke-Ungerer, Eva / Blell, Gabriele / Vences, Ursula (eds.): English-Español: Vernetzung im kompetenzorientierten Spanischunterricht. Stuttgart: ibidem. 315-328.

Lamb, Martin (2011). Future selves, motivation and autonomy in long term EFL learning trajectories. In: Murray, Garold / Gao, Xuesong / Lamb, Terry (eds.): Identity, Motivation and Autonomy in Language Learning. Bristol: Multilingual Matters. 177-194.

Lantolf, James P. (ed.) (2000). Sociocultural theory and second language learning. Oxford: Oxford University Press.

Lantolf, James P. (2006). Sociocultural Theory and L2. State of the Art. Studies in Second Language Acquisition (SSLA) 28: 67-109.

Lantolf, James P. / Thorne, Steven L. (2006). Sociocultural theory and the genesis of second language development. Oxford: Oxford University Press.

Larsen-Freeman, Diane (1997). Chaos/complexity science and second language acquisition. Applied Linguistics 18/2: 141-165.

Larsen-Freeman, Diane (2011). A complexity theory approach to second language development/acquisition. In: Atkinson, Dwight (ed.): 48-72.

Larsen-Freeman, Diane / Cameron, Lynne (2008). Complex Systems and Applied Linguistics. Oxford: Oxford University Press.

Lave, Jean / Wenger, Etienne (1991). Situated learning. Legitimate peripheral participation. Cambridge: Cambridge University Press.

Mercer, Sarah (2011). Understanding learner agency as a complex dynamic system. System 39: $427-$ 436. 
Meyer, Meinert A. I Reinartz, Andrea (eds.) (1998). Bildungsgangdidaktik. Denkanstöße für pädagogische Forschung und schulische Praxis. Opladen: Leske \& Budrich.

Murray, Garold / Gao, Xuesong / Lamb, Terry (eds.) (2011). Identity, Motivation and Autonomy in Language Learning. Bristol: Multilingual Matters.

Norton, Bonny (2000). Identity and language learning. Gender, ethnicity and educational change. Harlow: Pearson Education.

Norton, Bonny (2001). Non-participation, imagined communities and the language classroom. In: Breen, Michael P. (ed.): Learner contributions to language learning: new directions in research. Harlow: Pearson Education. 159-171.

Norton, Bonny / McKinney, Carolyn (2011). An Identity Approach to Second Language Acquisition. In: Atkinson, Dwight (ed.): 73-94.

Norton, Bonny; Toohey, Kelleen (2002). Identity and language learning. In: Kaplan, Robert B. (ed.): The Oxford Handbook of Applied Linguistics. Oxford: Oxford University Press. 115-123.

Norton, Bonny / Toohey, Kelleen (2011). Identity, language learning and social change. Language Teaching 44/4: 412-446.

Oevermann, Ulrich (1991). Genetischer Strukturalismus und das sozialwissenschaftliche Problem der Entstehung des Neuen. In: Müller-Dohm, Stefan (ed.): Jenseits der Utopie. Theoriekritik der Gegenwart. Frankfurt/M.: Suhrkamp. 267-336.

Oxford, Rebecca L. (2011). Meaning-making, border-crossing, complexity, and new interpretive techniques: expanding our understanding of language learner narratives. Zeitschrift für Fremdsprachenforschung 22/2: 221-241.

Paiva, Vera L. M. de O. (2011). Identity, motivation and autonomy in Second Language Acquisition from the perspective of Complex Adaptive Systems. In: Murray / Gao / Lamb (eds.): 57-72.

Pavlenko, Aneta / Lantolf James P. (2000). Second language learning as participation and (re)construction of selves. In: Lantolf, James P. (ed.): Sociocultural theory and second language learning. Oxford: Oxford University Press. 155-177.

Ricento, Thomas (2005). Considerations of identity in L2 learning. In: Hinkel, Eli (ed.): Handbook of Research in Second Language Teaching and Learning. Mahwah, NJ: Lawrence Erlbaum. 895-911.

Sade, Liliane A. (2011). Emerging selves, language learning and motivation through the lens of chaos. In: Murray / Gao / Lamb (eds.): 42-56.

The New London Group (1996). A pedagogy of multiliteracies:. In: Harvard Educational Review 66/1. Online:http://wwwstatic.kern.org/filer/blogWrite44ManilaWebsite/paul/articles/A_Pedagogy_of_ Multiliteracies_Designing_Social_Futures.htm [last retrieval (24.07.2013)].

Ushioda, Ema (2009). A person-in-context relational view of emergent motivation, self and identity. In: Dörnyei / Ushioda (2009a): 215-228.

Ushioda, Ema (2011). Language learning motivation, self and identity: current theoretical perspectives. Computer Assisted Language Learning 24/3: 199-210.

van Geert, Paul (2008). The dynamic systems approach in the study of L1 and L2 acquisition: an introduction. The Modern Language Journal 92/2: 179-199.

Wenger, Etienne (1998). Communities of practice. Learning, meaning, and identity. Cambridge: Cambridge University Press. 
Wimmer, Michael (2003). Ruins of Bildung in a Knowledge Society: Commenting on the debate about the future of Bildung. Educational Philosophy and Theory 35/2: 167-187. 


\title{
Identity Construction in Adult Learners of English for Specific Purposes (ESP): Exploring a Complex Phenomenon
}

\author{
Martina Weyreter / Britta Viebrock (Frankfurt/Main)
}

\begin{abstract}
Der vorliegende Beitrag untersucht die Zusammenhänge zwischen Fremdspracherwerb und der Konstruktion von Identität im spezifischen Lernkontext erwachsener Fachenglischlerner in Deutschland. Da Identitätskonstruktion in Sprachlernkontexten bisher hauptsächlich im Hinblick auf Migration, Mehrsprachigkeit bzw. Studienaufenthalte im Ausland diskutiert wurde, erscheint der Kontakt zu Muttersprachlern noch immer als unabdingbare Voraussetzung für erfolgreichen Spracherwerb. Solche Theorien können jedoch Identitätskonstruktion in einem Fachsprachlernkontext, in dem die Entwicklung von Berufsidentität und die Anwendung des Englischen als lingua franca auf internationaler Ebene Vorrang haben, nicht adäquat darstellen. Der vorliegende Beitrag stellt daher ein neues, kontextspezifisches Modell vor, das diesem besonderen Lernkontext Rechnung trägt. Der Beitrag schließt mit Vorschlägen zur Umsetzung in die Praxis.
\end{abstract}

The aim of this paper is to explore identity construction in adult learners of English for Specific Purposes (ESP) in Germany and to lay the conceptual foundations for further research on the construct of identity in the ESP context. As research on language and identity has mainly concentrated on contexts relating to migration, plurilingual societies and sojourns abroad, contact with a community of L1 speakers is invariably seen as the essence of identity construction in language learning. However, this seems hardly relevant to German ESP learners in a globalised economic environment, who are instead more likely to aspire to an enhanced professional identity where they use English as a lingua franca in an international context with other L2 speakers. By re-interpreting existing models of identity, a new context-specific model is derived to integrate factors that adequately reflect the language learning reality of adult ESP learners in Germany and their negotiation of identity. An outlook on practical applications of the new model is given.

\section{Introduction}

In the course of increasing migration flows and processes of globalisation, identity and identity construction have come into the focus of attention in foreign language education and research. As identity has been explored from many angles, it is difficult to define the construct; however, for the purpose of this study, it is assumed that identity is self-perceived and self-constructed, that it can and indeed does change throughout an individual's life, and that multiple identities can co-exist in one individual.

On the whole, the negotiation of identity in language learners has most frequently been examined in contexts where learners are in direct contact with 
L1 ('native') speakers, either as members of multilingual societies or as foreign language learners sojourning in a location where the target language is spoken as an L1. This paper aims to explore identity in a different learning context, that of adult learners of English for Specific Purposes (ESP) in Germany. Compared to foreign language education at school, ESP courses for adults (e. g. Business English, English for Academic Purposes, English for Science and Technology, etc.) tend to have a much more functional purpose and a clear vocational orientation where language skills are usually acquired for communication in lingua franca contexts rather than for communication with L1 speakers. Although a large percentage of adult learners of English in Germany attend ESP courses, their perspective of identity and identity construction processes through language learning has remained largely unexplored.

This paper begins by outlining relevant aspects of identity construction in ESP and subsequently presents a short overview of the various scientific approaches to identity and language learning, comparing and contrasting previous research from the fields of anthropology, social psychology, and sociolinguistics. While these approaches seem to take into account aspects of both individual identity and group identities in language learning, they invariably see contact with an English-speaking community and culture as the essence of identity construction in this context, and fail to account for learners for whom achieving native-like language competence and familiarity with another culture may take low priority. Instead, as this paper suggests, ESP learners are more likely to aspire to an enhanced professional identity where they are able to use English as a lingua franca, improve their career prospects and position themselves as true global players, without uprooting their stable core identity.

A new model of identity construction in the ESP context is therefore proposed, adopting some aspects known from previous models but modifying and adding to them to reflect the realities of ESP learning among adults in Germany today.

\section{Identity construction in ESP}

To understand issues of identity and identity construction among adult ESP learners in Germany, it is necessary to gain an overview of their learning environment and their reasons for embarking on ESP courses, as well as an understanding of ESP itself. The concept of English for Specific Purposes was first developed in the 1960s and 70s (cf. Howatt/Widdowson 2004; Swales 2000) when, in times of increasing globalisation, more and more working adults needed to use English for work-related communication and discovered that the kind of English they had learnt at school was insufficient for their 
requirements. Specialised ESP courses emerged, focusing e.g. on Business English, English for Academic Purposes or English for Science and Technology (Hutchinson/Waters 1987; Swales 2000), although highly specialised professional courses in Aviation English, English for Law Enforcement, English for the Pharmaceutical Industry or the Financial Sector also became available.

The study of ESP may include not only lexical and grammatical aspects of work-related language but also cultural insights into the respective profession, and train learners in practical skills (e. g. telephoning or giving presentations in English) so as to enable them to function competently as a member of the discourse community in their field. In particular, they may need English to read international publications (in print or online), communicate with international business contacts, attend conferences or trade fairs, design their own company website in English or even - for scientists and academics from all fields - to publish in international English-language journals. Yet ESP is equally of interest to students in higher education and to young people in vocational training because it is a valuable skill to have when entering the job market for the first time. At present, ESP is in fact the fastest growing sector in English language teaching (Vogt 2011) and - particularly in the Frankfurt/M. area of Germany (where the authors of this chapter are located) with its strong economic base in industry and finance - there are now more EFL teachers on the regional adult education market teaching ESP than general English (ELTAF 2008). Presumably, the situation is similar in other highly industrialised countries or regions (e. g. North East China, Li 2013).

However, when looking at this booming market for ESP, it becomes obvious that learning objectives and learner motivation have shifted. As an international lingua franca used worldwide in business, academia and many other communicative environments, English has become an increasingly prestigious language which by now has more non-native than native speakers worldwide (cf. Kachru 1992). It follows that adult learners around the world today study English not in order to travel to an English-speaking country or even to communicate with L1 speakers, but mainly to use it as a lingua franca in international work-related contexts with other L2 speakers. Eschmann (2005) found that in fact only $32 \%$ of learners of English at a German Volkshochschule (community adult education institute) had ever been to an English-speaking country, compared to e. g. $85 \%$ of learners of Italian who had been to Italy.

Consequently, existing models and research approaches to identity construction in foreign language learning will have to be modified or reinterpreted to suit these changed objectives. 


\section{$3 \quad$ Identity and foreign language learning: A variety of approaches}

In order to define the construct of identity and to understand its role in foreign language learning, particularly in ESP, it is first of all necessary to explore and juxtapose a number of past and present research approaches relating to the subject.

\section{Anthropology}

The idea of a connection between language and identity, particularly when it comes to the acquisition of a second or foreign language, has been of interest to anthropologists, social psychologists, sociolinguists and educators alike. Historically, anthropological theories of a connection between language and national identity reach back as far as Herder and Humboldt (cf. Risager 2006). In the early part of the 20th century, anthropologists such as Boas, Sapir and Whorf successively developed the linguistic relativity theory which claims that "the structure of a language determines a native speaker's world view. Different languages are assumed to lead to different world views" (Carroll 1999: 364) and, consequently, "learning a language changes the way a person thinks" (ibid.: 365). Despite a lack of empirical evidence, these theories are at the root of a research tradition reaching into the $20^{\text {th }}$ and $21^{\text {st }}$ century.

\section{Social psychology}

Around the early 1960s, a research tradition on attitude and motivation in second language acquisition (SLA) emerged in the field of social psychology. Canadian scholars, trying to investigate why English and French speakers in Canada were often unsuccessful at learning and using each other's languages, showed that social psychological variables such as an open and friendly attitude towards the speakers of the other language (the L2 community) and the degree of identification with this community could have a strong positive effect on language acquisition (Gardner/Lambert 1959). In other words, to acquire a second language to a high level, the learner had to integrate this language and its community into his or her individual identity, to make it a part of his/her very self. Based on the social identity theory of intergroup behaviour (Giles/Byrne 1982; Tajfel/Turner 1979), Gardner (1985) eventually developed the socioeducational model of second language acquisition which was highly influential because it stressed the importance of individual difference variables in SLA, not only integrativeness (identification with a group of proficient speakers) but also attitudes and motivation, and showed their interrelation.

In this manner, the social psychological approach was the first to move away from the hitherto dominant idea of innate language aptitude as the main 
predictor of achievement at second or foreign language acquisition (FLA) (Carroll/Sapon 1959), and to include factors specific to the individual's personal identity. In addition, Gardner's socio-educational model suggests that language learning may yield 'non-linguistic outcomes', which are of great interest when investigating identity changes and identity construction in foreign language acquisition. Despite admitting that "the nature of these proposed changes is not well detailed" (Gardner 1985: 143), Gardner (ibid.: 84) defines them as byproducts of the language learning experience: "If attitudes and motivation influence how well someone learns a second language, is it not equally possible that the experience of learning a second language influences attitudes and motivation?" This circular interpretation of the socio-educational model illustrates the idea that many of the variables influencing language study in the first place may later resurface as - ideally positive - non-linguistic outcomes of language study. Empirical evidence seems to substantiate these claims, at least for students on intensive immersion courses in the target country and in migration contexts, i. e. where there is contact with an L2 community (Block 2007; MacIntyre et al. 2003; Pavlenko 2006). However, as described above, the ESP learning context in Germany which the present paper aims to examine usually offers little or no direct contact with an L2 community. In fact, with multicultural societies being the norm in our globalised $21^{\text {st }}$ century, the cultural dichotomy presented by the intergroup model (L1 vs. L2 community) may be considered irrelevant in a context where a foreign language is learnt for purposes other than to communicate with a community of native speakers. Consequently, one must ask how learners can possibly perceive integrativeness when there is no longer an L2 community to integrate and identify with! For all these reasons, it is necessary to modify and update the existing model to account for a different, changed reality (see chapter 4).

At the same time, Gardner (1985) contrasted integrative orientation towards learning a language (which may affect identity) with instrumental orientation. The latter describes an individual's motivation to learn another language fuelled by the prospect of external rewards such as valuable qualifications, employment prospects, promotion or a higher salary. Clearly, such external rewards can positively affect self-identity and are likely to be highly relevant to adult learners acquiring ESP for professional purposes. Dörnyei (2005, 2009) and Ushioda/Dörnyei (2009) connect language learning to an individual's quest for the 'ideal self' (fuelled by personal hopes, aspirations and wishes) out of a choice of 'possible selves', as well as to a quest for societal status by fulfilling the expectations of others: the 'ought-to self' (what one believes one should be). This is in line with Oerter/Dreher (2008) who describe a natural human desire to reduce any discrepancy between current and ideal self. Hence, the 'ideal L2 self' (Ryan 2009) might be a person who speaks English confidently, who has an interesting job where they communicate in English on an international level, 
who is successful and admired - all of which forms part of his/her self-identity. Interestingly, studies in countries as varied as Hungary (Czisér/Kormos 2009), Japan (Ryan 2009), and Iran (Taguchi et al. 2009) have confirmed these theories, taking into account not only the individuals' personal aspirations but also the diverse cultural contexts they may live and study in, especially with respect to the 'ought-to self'.

\section{Sociolinguistics}

While most of the social psychological theories described above focus on the individual self, sociolinguistic research emphasises the fact that human beings are social beings and do not exist in a vacuum. Hence, sociolinguists define individual identity as being constructed out of social identity and, as a consequence, out of many different group identities (Joseph 2004). In other words, people are not thought to interact and communicate purely as individuals but as members of the groups they belong to and identify with (Giles et al. 2010) and where they attach emotional significance to such membership (Benwell/Stokoe 2006). Multiple identities of an individual may include e. g. ethnic identity, national identity (Joseph 2004) or cultural identity (Thomas 1992) amongst others - but also, on a lower degree of abstraction, familial identity (Giles/Johnson 1981) or identity as a member of a sports club or similar social group. Such a multitude of identities is thought to be necessary because each individual has roles to play with regard to others, e. g. their parents, spouse, colleagues, boss, or friends (Joseph 2004). What is more, these identities are not fixed but constantly self-created and re-negotiated in the act of communication (Giddens 1991).

This raises the question as to which identities can change or be actively reconstructed when learning a foreign language, and, more specifically, English for Specific Purposes. Because of the different scientific approaches to identity, it is necessary to define the construct in a way that enables us to carry out further research relating to a specific learning context and environment. For the purpose of this research, it can therefore be summarised that self-identity

- is self-perceived, not objective but subjective,

- is not assigned but can change and be self-constructed,

- consists of multiple identities that co-exist and complement each other, e. g. individual identities and group identities,

- is created in communication with other individuals or groups,

- is "an ongoing lifelong project" (Block 2006: 35) as its construction and reconstruction have become a fact of life in today's globalised world,

- includes an awareness of what one is compared to what one would like to be. 
Of course, despite the necessity for ongoing change, every human being wishes to retain a stable core identity in a quest for security and safety in their life (Norton 1997). So when it comes to foreign language learning, individuals may experience a "pull in opposite directions" (Jenkins 2006: 87): the attraction of English as a global language of communication that is sure to bring instrumental achievement, self-confidence and the respect of others, versus the safety and belonging offered by their L1 language of identification; modern-time identity flux versus desire for stability. This may have diverse effects on the learner, such as fear of assimilation (Clément 1980), resistance to speak the L2 in an attempt to protect one's identity as a competent and articulate individual (Worth 2007), or a feeling of simultaneously embracing and rejecting the language learning experience (Granger 2004). In other words, learners will most likely refuse to 'change' identity in the sense of giving up one thing to obtain another. Instead, language learning ought to offer a positive opportunity to add something to what one already has, thereby enriching the total and allowing the learner to form an integrated self where all identities coexist peacefully.

Hence, learning about and experimenting with new identities in the course of language learning may be an attractive prospect, but - as explained above - ESP learners in Germany are unlikely to identify with native English speakers and their communities in any sense that could be called integrativeness. The reason for this is simply that English-language communicators and communicative situations, especially in the world of work (which ESP learners' objectives are focused on) are becoming increasingly heterogeneous and detached from any clearly-defined national community (Dörnyei et al. 2006). So the question is how can there be identity construction in FLA when there is no longer an L2 community to identify with and when, rather than being geographically remote, such a community has in fact ceased to exist? Who will provide a tangible group identity or set an example students might strive to emulate? Or is motivation to learn ESP strictly limited to instrumental factors, i. e. the wish to learn a skill that may bring about promotion at work, a higher income, and the respect of others? And could purely instrumental motivation, although very likely to be present in ESP students, ever affect something so personal and emotional as self-identity?

\section{Towards a new context-specific model of ESP identity}

As language learning contexts change over time to reflect modern-day requirements and the specific needs of learners, it is obvious that the underlying constructs and research contexts also need to adjust, in order to keep pace with $21^{\text {st }}$ century realities. However, it has been shown that the existing concepts and models of identity construction in FLA are no longer applicable in some context-specific $21^{\text {st }}$ century language learning environments. Therefore, a new 
model is needed in order to analyse and explain the nature of identity construction in adult ESP learners in Germany, to include newly relevant factors other than the ones described in the existing social psychological and sociolinguistic literature. This does not mean having to discard the concept of the integrative motive altogether, as some scholars have claimed (Coetzee-van Rooy 2006; Ushioda 2006), but it does create a need to redefine this motive to suit a new context. If there is no longer a tangible ethnic L2 community whose identity an ESP student may wish to integrate with, its place must be taken by something else: for example, a professional community with a professional identity.

So far, professional identity development (e. g. Glaser-Segura et al. 2010; Gordon/Luke 2012; Helmich et al. 2010), despite being a popular research topic in psychology, has rarely been linked to language. Yet it is a construct highly relevant to ESP as, after all, learners would expect to use their newly-acquired linguistic skills in a professional context. However, the quest for professional identity is quite distinct from purely instrumental considerations such as obtaining a good job, promotion and financial rewards. It can be defined as the integration of knowledge, skills, self-concept, values, attitudes and autonomy (Glaser-Segura et al. 2010), all of which involves a characteristic style of thinking based on solid knowledge of one's discipline, coupled with self-motivation and enthusiasm (Farrell 2000). In other words, it is assumed that one's profession - and with it, membership of a professional 'community of practice' (Wenger 1998) - is an identity-giving aspect of one's life.

So how is identity constructed and reconstructed with regard to one's profession and career? First of all, an individual's quest for the 'ideal self' (Ushioda/Dörnyei 2009, see above) is likely to include an ideal professional self, and if a command of ESP forms a desirable part of this professional self, the individual is likely to be motivated to acquire ESP to a high level. Yet professional identity is not purely how individuals experience themselves as competent workers and fulfil their personal professional ambitions. It is also constructed socially via the perception of oneself as a member of a professional community, be it real or imagined. Such a community of practice may be characterised by "mutual engagement, a joint enterprise and a shared repertoire" (Wenger 1998: 73) whilst also sharing and maintaining a historical and social context, routines, structures, anecdotes and humour in everyday communication in the working environment.

This is also the point where language becomes relevant: To be a member of a professional group, one needs to share this group's language and participate in the domain-specific discourse; in fact, language can be used by this group to "differentiate between in-group and out-group members" (Glaser-Segura et al. 2010: 200). So, if the language of one's current or desired professional 
community is English - or ESP, to be more precise - then successful acquisition of ESP is the entry ticket to this community and therefore a highly relevant factor of self-identity and identity construction. Even if this professional community is not (or not yet) present in a learner's immediate environment, they may already imagine themselves as a future member. For example, GlaserSegura et al. (2010) believe that professional socialisation begins as soon as professional studies are taken up.

It may be said, therefore, that the presence of an integrative motive in learners of ESP is of great importance to ESP linguistic achievement and successive socialisation in an international professional community or working environment. Hence, Gardner's and Lambert's (1959: 271) original definition of integrativeness as "a willingness to be like valued members of the language community" still applies in the modern-day ESP context, even though the L2 community in question is no longer a national one with its cultural characteristics, but a professional one with its own cultural environment. ESP learning can thus be both instrumentally and integratively motivated, without any inherent dichotomy between the two (cf. Ryan 2006), as integration into a professional community and instrumental achievement become closely interrelated and even interdependent.

Finally, an added factor of identity construction important to learners is the global aspect of ESP. At least for learners in Germany, an English-speaking professional community is likely to be an international one where this language is used as a lingua franca. Its members may not be in the same place geographically, but may otherwise share all the characteristics of a professional community so that - depending on the working environment - they are likely to identify as members of a global professional community (cf. Dörnyei et al. 2006) in a real or imagined sense. The learner has become a 'global player' who uses a prestigious language that makes them stand out as an individual yet identifies them as a member of an in-group. Of course, any kind of identity construction in ESP is likely to depend on an individual's personal learning background as well, and there tends to be a great amount of difference between individual learners. Identity construction is highly likely to be influenced by how much time and effort an individual invests in ESP learning and how much personal importance he or she attaches to this effort. However, it is safe to say that most of them would feel attracted to the idea of eventually becoming a member of a community of globally involved professionals.

In summary, and taking into account the multiple identities of relevance to ESP acquisition, it is proposed that the following six factors are likely to play a part in an ESP learner's identity construction (cf. figure 1):

- 'Instrumental': L2 being useful at work and promising economic gain 
- 'Professional': individual identity as a professional, membership of real and imagined professional L2 communities of practice

- 'Individual': intrinsic quest for the ideal self, positive differentiation of the speaker

- 'Global': global identity, membership of a global community, prestige, positive differentiation of the language

- 'Personal learning background': social and historical learning background and learner's relationship to the language, investment in language learning

- 'L1 identity maintenance': managing multiple cultural and social identities, retaining a stable core identity while exploring L2 culture and identities

As the model shows, six factors are believed to affect identity construction in ESP learners, and all these factors are likely to relate to each other. With multiple identities present or emerging simultaneously in an individual, identity construction in ESP learners is certainly a complex issue worthy of closer investigation in the future. The complexities include the need to balance individual versus group identities as well as the quest for membership of a professional L2 community of practice versus the need to maintain an integrated self.

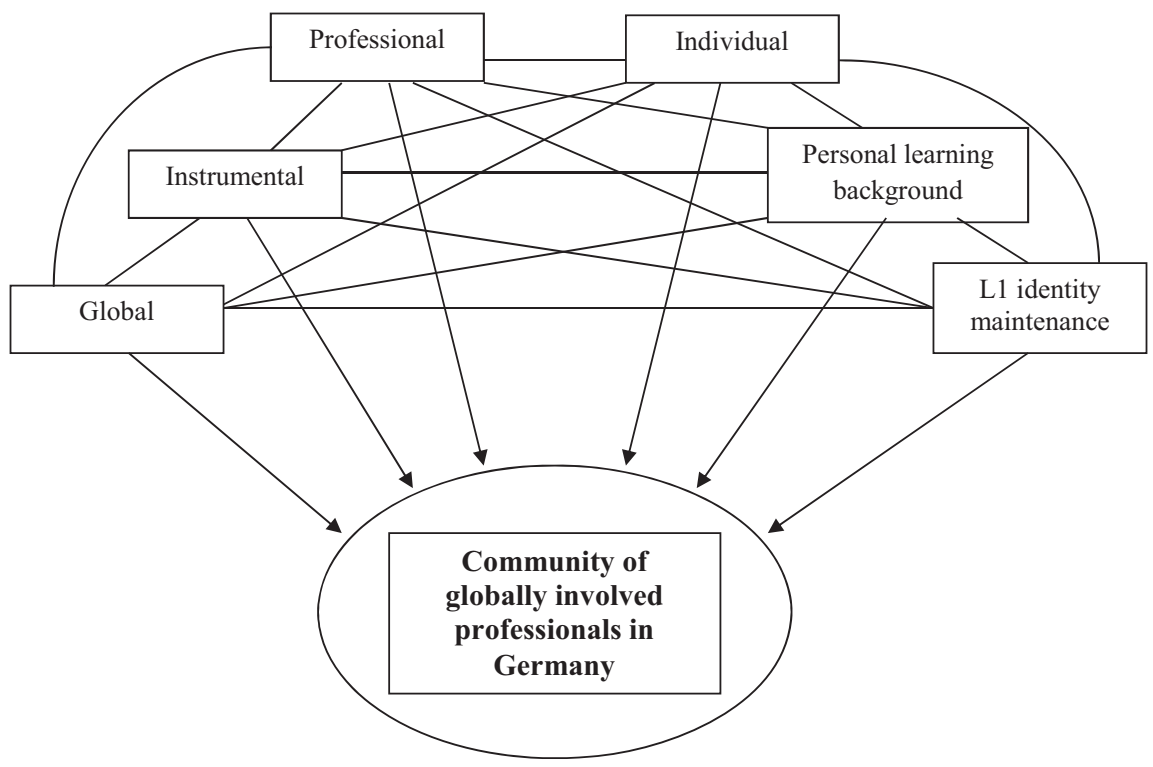

Figure 1: Factors playing a part in ESP learners' identity construction 


\section{$5 \quad$ Practical application and research outlook}

Clearly, empirical research is needed to validate this model and to possibly identify additional factors that may exist, as well as to determine if and how all the factors described may interrelate. A research project is therefore planned whereby ESP learners from different fields (e. g. Business English, Academic English, Technical English, and other less often studied kinds of ESP) will be asked, both by questionnaire and by interview, about their personal perceptions of identity and identity construction with a view to learning and using ESP. A mixed methods approach (e. g. Ecarius/Miethe 2011) using both quantitative and qualitative research methods will then help to analyse and provide a clearer picture of how identity construction in ESP can take place in various individuals and what the main factors are in promoting or hindering such construction.

It seems obvious, though, that ESP acquisition and identity construction are likely to go hand in hand in some way. So, from a practical point of view, if linguistic achievement is to be promoted, it may be argued that identity development is equally desirable and deserves to be promoted by language educators. Of course, this can be a sensitive issue if one is to avoid manipulation and intrusion into a learner's personal space. Hence, language educators may be best advised to promote identity construction and development simply by providing opportunities for students to reflect on and discuss identity issues while they learn, and by raising awareness. It is not expected that identity construction will per se depend on a teaching method or methods as the active ingredient in this process is thought to be the student, not the teacher - although he or she can offer help and encouragement.

Practical classroom activities to promote identity construction and development may include raising awareness of the instrumental benefits of knowing ESP, discussing the pros and cons of professional and lifestyle choices, doing case studies of working professionals abroad and possibly promoting social contact with such professionals, inviting speakers who are experts in the field, exploring opportunities for language practice outside the classroom, and comparing German and English-speaking professional cultures with a view to encouraging students' desire to construct professional identity to the same level in L2 as in L1. In addition, identity issues may be of relevance in language counselling (a service that many schools and universities offer) and counsellors should therefore be aware of them. Testing the effectiveness of these practical activities could of course also be the topic of further research.

In this manner, identity construction will ideally become a powerful tool to aid the L2 learning process and help ESP students on their path to becoming global professionals. 


\section{References}

Benwell, Bethan / Stokoe, Elizabeth (2006). Discourse and identity. Edinburgh: Edinburgh University Press.

Block, David (2006). Identity in applied linguistics. In: Omoniyi, Tope / White, Goodith (eds.): Sociolinguistics of identity. London: Continuum. 34-49.

Block, David (2007). Second language identities. London: Continuum.

Carroll, David W. (1999). Psychology of language. 3rd edition. Pacific Grove, CA: Brooks/Cole.

Carroll, John B. / Sapon, Stanley M. (1959). Modern Language Aptitude Test (MLAT). New York: Psychological Corporation.

Clément, Richard (1980). Ethnicity, contact and communicative competence in a second language. In: Giles, Howard / Robinson, W. Peter / Smith, Philip M. (eds.): Language: social psychological perspectives. Oxford: Pergamon Press. 147-154.

Coetzee-van Rooy, Susan (2006). Integrativeness: untenable for world Englishes learners? World Englishes 25: 437-450.

Czisér, Kata / Kormos, Judit (2009). Learning experiences, selves and motivated learning behaviour: A comparative analysis of structural models for Hungarian secondary and university learners of English. In: Dörnyei, Zoltán / Ushioda, Ema (eds.): Motivation, language identity and the L2 self. Bristol: Multilingual Matters. 98-119.

Dörnyei, Zoltán (2005). The psychology of the language learner. Mahwah, NJ: Lawrence Erlbaum Associates.

Dörnyei, Zoltán (2009). The L2 motivational system. In: Dörnyei, Zoltán / Ushioda, Ema (eds.): Motivation, language identities and the L2 self. Bristol: Multilingual Matters. 9-42.

Dörnyei, Zoltán / Csizér, Kata / Németh, Nóra (2006). Motivation, language attitudes and globalization. Clevedon: Multilingual Matters.

Ecarius, Jutta / Miethe, Ingrid (eds.) (2011). Methodentriangulation in der qualitativen Bildungsforschung. Opladen: Barbara Budrich.

English Language Teachers' Association of Frankfurt (2008). ELTAF members' survey. Online: www.eltaf.de (last retrieval [13.04.2013]).

Eschmann, Detlef (2005). Englischlernende an Volkshochschulen. In: Ahrens, Rüdiger / Weier, Ursula (eds.): Englisch in der Erwachsenenbildung des 21. Jahrhunderts. Heidelberg: Universitätsverlag Winter. 263-288.

Farrell, Lesley (2000). Ways of doing, ways of being: Language, education and 'working' identities. Language and Education 14/1: 18-36.

Gardner, Robert C. (1985). Social psychology and second language learning. London: Edward Arnold.

Gardner, Robert C. / Lambert, Wallace E. (1959). Motivational variables in second language acquisition. Canadian Journal of Psychology 13: 266-272.

Giddens, Anthony (1991). Modernity and self-identity. Cambridge: Polity Press. 
Giles, Howard / Byrne, Jane L. (1982). An intergroup approach to second language acquisition. Journal of Multilingual and Multicultural Development 3/1: 17-40.

Giles, Howard / Johnson, Patricia (1981). The role of language in ethnic group relations. In: Turner, John C. / Giles, Howard (eds.): Intergroup behaviour. Oxford: Blackwell. 199-243.

Giles, Howard / Reid, Scott / Harwood, Jake (eds.) (2010). The dynamics of intergroup communication. New York: Lang.

Glaser-Segura, Daniel A. / Mudge, Suzanne / Bratianu, Constantin / Dumitru, Ionela (2010). Development of professional identity in Romanian business students. Education \& Training 52/3: 198-213.

Gordon, Cynthia / Luke, Melissa (2012). Discursive negotiation of face via email: Professional identity development in school counseling supervision. Linguistics and Education 23/1: 112-122.

Granger, Colette A. (2004). Silence in second language learning. Clevedon: Multilingual Matters.

Helmich, Esther / Derksen, Els / Prevoo, Mathieu / Laan, Roland / Bolhuis, Sanneke / Koopmans, Raymond (2010). Medical students' professional identity development in an early nursing attachment. Medical Education 44/7: 674-682.

Howatt, Anthony P. R. / Widdowson, Henry G. (2004). A history of English language teaching. Oxford: OUP.

Hutchinson, Tom / Waters, Alan (1987). English for Specific Purposes. Cambridge: CUP.

Jenkins, Jennifer (2006). English pronunciation and second language speaker identity. In: Omoniyi, Tope / White, Goodith (eds.): Sociolinguistics of identity. London: Continuum. 75-91.

Joseph, John E. (2004). Language and identity: National, ethnic, religious. Basingstoke: Palgrave Macmillan.

Kachru, Braj (1992). The Other Tongue: English across cultures. Champaign, IL: University of Illinois Press.

Li, Lixin (2013). Theoretical base and problems in Business English Teaching in China. Online: http://www.esp-world.info/Articles_8/Li.htm (last retrieval [08.04.2013]).

MacIntyre, Peter D. / Baker, Susan C. / Clément, Richard / Donovan, Leslie A. (2003). Talking in order to learn: Willingness to communicate and intensive language programs. The Canadian Modern Language Review 59/4: 589-607.

Norton, Bonnie (1997). Language, identity, and the ownership of English. TESOL Quarterly 31/3: 409-429.

Oerter, Rolf / Dreher, Eva (2008). Jugendalter. In: Oerter, Rolf / Montada, Leo (eds.): Entwicklungspsychologie. 6. Aufl. Weinheim: Beltz. 271-332.

Pavlenko, Aneta (2006). Bilingual selves. In: Pavlenko, Aneta (ed.): Bilingual minds. Clevedon: Multilingual Matters. 1-33.

Risager, Karen (2006). Language and culture. Clevedon: Multilingual Matters. 
Ryan, Stephen (2006). Language learning motivation within the context of globalisation: An L2 self within an imagined global community. Critical Enquiry in Language Studies 3/1: 23-45.

Ryan, Stephen (2009). The ideal L2 self and Japanese learners of English. In: Dörnyei, Zoltán / Ushioda, Ema (eds.): Motivation, language identities and the L2 self. Bristol: Multilingual Matters. 120-143.

Swales, John M. (2000). Languages for specific purposes. Annual Review of Applied Linguistics 20: 59-76.

Taguchi, Tatsuya / Magid, Michael / Papi, Mostafa (2009). The L2 motivational self system among Japanese, Chinese and Iranian learners of English: A comparative study. In: Dörnyei, Zoltán / Ushioda, Ema (eds.): Motivation, language identity and the L2 self. Bristol: Multilingual Matters. 66-97.

Tajfel, Henri / Turner, John C. (1979). An integrative theory of intergroup conflict. In: Austin, William C. / Worchel, Stephen (eds.): The social psychology of intergroup relations. Monterey, CA: Brooks Cole. 33-47.

Thomas, Alexander (1992). Grundriß der Sozialpsychologie, Band 2: Individuum - Gruppe Gesellschaft. Göttingen: Hogrefe.

Ushioda, Ema (2006). Language motivation in a reconfigured Europe: Access, identity, autonomy. Journal of Multilingual and Multicultural Development 27/2: 148-161.

Ushioda, Ema / Dörnyei, Zoltán (2009). Motivation, language identities and the L2 self: A theoretical overview. In: Dörnyei, Zoltán / Ushioda, Ema (eds.): Motivation, language identities and the L2 self. Bristol: Multilingual Matters. 1-8.

Vogt, Karin (2011). Fremdsprachliche Kompetenzprofile. Tübingen: Narr.

Wenger, Etienne (1998). Communities of practice: Learning, meaning and identity. Cambridge: CUP.

Worth, Robin A. (2007). Learner resistance in the university foreign language classroom. The University of Wisconsin, Madison. UMI Dissertations Publishing, No. 3245650. 


\title{
Constructing German Learner Identities in Online and Offline Environments
}

\author{
Alice Chik (Hong Kong)
}

This article discusses the findings from an on-going school-based online exchange project between students from Hong Kong and Berlin. The former are proficient and confident English speakers, and have taken on the challenge to study German as an additional foreign language in after-school classes. While English cultural and popular cultural products are freely circulated in the Hong Kong media, there is very limited access to German cultural and popular cultural products. When it comes to language learning, it is possible to view the participants as having a split have and have-not identity between the two languages. Yet, how do participants see themselves as learners and users of German? Drawing on online writing practice artefacts, self-portraits, and interview data from the projects, I will argue that sociocultural-economic binaries do not necessarily define or limit students' creativity and ingenuity in appropriating available resources in language learning. This article will conclude with implications for modern language teaching in secondary school settings and language teacher education.

\begin{abstract}
本文討論一個以香港和柏林學生之間在線交流研究項目的結果。英語文化和流行 文化產品在香港媒體是廣泛流通, 但德語文化和流行文化產品在香港媒體的流通 則非常有限。此研究項目中的香港學生均是熟練和自信的英語作為第二語言學習 和使用者, 並接受在課後班學習德語作為一個附加外語的挑戰。以第二或外語語 言學習來說, 這些學生可以說是擁有兩種分裂的語言身份認同。這些德語學生又 如何看待自己作為德語學習者和使用者的身份? 本文採用的數據包括在線寫作例 子、自畫像和訪問。我會在文中闦釋社會、文化、或經濟條件不一定定義或限制 學生的創造力和劃撥語言學習資源。本文將總結此研究項目結果對現代語言教學 和語言教師培訓的蘊涵。
\end{abstract}

\section{$1 \quad$ Introduction}

Though there has been increasing interest in multilingualism in the last decade, especially in European contexts, the situation in East Asia might have moved in a different direction. In a global context in which English is fast becoming the only second or foreign language learned and used by many learners due to its lingua franca status, the sociocultural spaces for learning and using other modern and Asian languages are fast becoming more limited. While the teaching and learning of English is almost a given in many East Asian educational systems, as witnessed in the provision of English classes in formal curricula, additional modern languages are usually not offered (Nunan 2003). In Hong Kong, English is a required academic subject starting from Primary One and students also have to pass a public examination aimed at the assessment of their English skills at the end of Secondary Six in order to progress to local tertiary Bachelor's Degree programmes. However, modern languages such as French or German are usually not offered at schools at all. Many tertiary students, 
especially those reading for a discipline in Humanities, usually take modern or Asian languages to fulfill the foreign language requirement. At secondary or primary levels, students will have to pay for private tuition if they wish to learn an additional language. In East Asian contexts, especially in Hong Kong, it is also a challenge for learners to construct personal spaces to effectively and efficiently learn a third language, an issue addressed by Humphreys/Spratt (2008) who claim that there is almost no social presence of modern languages in the community. It is then worthy to explore the learning and acquisition of languages other than English to further our understanding and challenges of modern language teaching in East Asia.

\section{The teaching and learning of a third language}

In the present chapter, I adopted Jessner's (2008: 18) definition of third language (L3) as "the third language that the speakers had contact with during her/his lifetime" to highlight the exposure a learner has towards a language. It is drastically different from Hammarberg's (2010: 97) definition of L3 as "a nonnative language which is currently being used or acquired in a situation where the person already has knowledge of one or more L2s in addition to one or more L1s". The latter definition highlights the complexity of multilingualism in contexts as a learner can simultaneously use and acquire an additional language to his/her existing knowledge of L1s and L2s. Given the social status of English in Asian contexts, Jessner's (2008) definition is more representative of L3 learning. Studies on the teaching and learning of a third language frequently focus on three scenarios: children growing up with three languages, bilingual children learning an L3, and bilingual migrant children learning an L3 in new socio-linguistic environments. One issue that Jessner (2008) raised was the isolation of the teaching of an L3 in the classroom regardless of increases in immersion programmes or content-based learning. L3 teaching and learning is essentially being treated as a separate subject and disconnected from other academic subjects.

This isolation of teaching an L3 as an academic subject is problematic in itself, but it could also lead to other relevant problems in language learning. Csizér/Kormos (2008) found that among the 1777 Hungarian learners (aged 13 and 14) surveyed, German-learning students had a lower level of linguistic selfconfidence than the English-learning students. They attributed the main factor to be students' limited exposure to German media products, even though German is the major language spoken in the neighbouring countries. The lower level of linguistic self-confidence in turn led to a lower motivation to learn German. Access to media products and artifacts, such as popular music and films, is found to be the main contributor to students' motivation to learn English (Csizér/Kormos 2009). Investigating foreign language learning motivation in 
Hong Kong, Humphreys/Spratt (2008) surveyed 259 undergraduates who were learning French, German and Japanese. They found that although the 65 students learning German had high integrative motivation to learn, they had very low 'linguistic self-confidence', and had a high level of anxiety as beginners. Yet, we should focus on the L2 status among the learners from Hong Kong. Originally proposed by Williams/Hammarberg (2009/1998, cited in Falk and Bardel 2010), the status factor of learners' L2 will directly impact the acquisition of the L3, as learners use a prior L2 as an orientation while learning an L3. This may be especially relevant in the East Asian contexts, as most students would have learned English as their L2, and they have greater and easier access to English media products than German media products. English is also being perceived as having a higher status, being the lingua franca in Asia.

\section{Digital practices and second and foreign language learning}

Dörnyei and Csizér (2005: 2) argue that "intercultural contact is both a means and an end in L2 studies", and for many L2 learners, the major means for intercultural contact could well be through their digital practices. The current generation of learners are users of Web 2.0 tools in their daily lives: they are on Twitter, Facebook, YouTube, blogs and wikis. The increasing use of Web 2.0 technologies in daily life is already part of popular cultural practices. There have now been a number of studies investigating out-of-class second and foreign language (SFL) learning in digital environments (Black 2007; Kuure 2011; Lamy/Hampel 2007; Lee/Barton 2009; Leppänen et al. 2009; Thorne et al. 2009). In this environment that offers various media, young people are actively orchestrating a range of literacies to make meaning, construct knowledge and identities with and through multiple media, a practice that O'Brien (2006) terms "multimediating". Literacy practices are blurred, or "dissolved" (Alvermann/Eakle 2007), especially in the digital age, because young people are actively "completing projects to help explain themselves, their interest, their pleasures, and the worlds they inhabit or would like to inhabit" (O'Brien 2006). When L2 learners are readily using different digital tools for SFL learning, there is a need to "examin[e] the social processes that hinder or facilitate the mobility of specific literacies across contexts" (Sheehy 2009: 144f.). This is especially relevant for the analysis of the transfer between L2 and L3 digital practices.

\section{$4 \quad$ Methodology}

The present article draws on a school-based online exchange project between secondary school students from Berlin and Hong Kong, however, this chapter only reports findings on the ways the Hong Kong participants viewed themselves as language learners of German. In Hong Kong, all students are required 
to take English as a compulsory subject from Primary One (First Grade), and they have to pass the English examination at the end of their secondary education in order to enter university. It is debatable whether English is a second or foreign language in Hong Kong, as English learning contexts vary greatly among secondary school students (Poon 2010). Participants from the present study were Secondary Three ( $9^{\text {th }}$ Grade) students $(\mathrm{N}=8$, all females) from a government-subsidized English-medium school. Studying in an English-medium school means that other than Chinese Language and Chinese History, all other subjects are taught in English. German is offered as an additional third language for selected students in an after-school curriculum. The offering of German is unusual for a school that runs a local curriculum. The school is located in an affluent neighbourhood and most students come from affluent socioeconomic family backgrounds. All participants have taken overseas leisure trips with their family or have joined study tours organized by the school. It is safe to assume that these participants come from more privileged backgrounds than the average secondary school student from Hong Kong.

However, coming from privileged backgrounds does not necessarily guarantee access to linguistic and cultural resources. English is listed alongside Chinese as an official language. All government and major corporation websites are bilingual in Chinese and English. According to the 2011 Population Census, $3.5 \%$ of the total population of about 7.2 million speak English as their usual language, with another $46.1 \%$ who speak it as another language. In addition to mass media in Chinese, people in Hong Kong also have free access to Englishlanguage mass media: two TV channels and three radio stations. The two local TV channels frequently broadcast popular contemporary American and British drama and sitcom programmes, for instance, CSI, Modern Family, and Downtown Abbey - to name but a few. In addition, TV viewers can subscribe to cable $\mathrm{TV}$ to watch premium channels like BBC, CNN, HBO, Fox and ESPN, etc. Films in English are the staples of Hong Kong cinemas. Locally published newspapers and magazines in Hong Kong English are also readily available for free or at very low cost. In the daily life of an average Hong Kong resident, English media products are easily accessible.

However, access to German in Hong Kong is much more limited. According to the German Federal Foreign Office, the estimate number of German citizens living in Hong Kong is about 3.000 (Federal Foreign Office 2012). Though German is offered in universities, it is usually not offered in government-funded or -subsidized local schools. Learners who are interested in learning German have to pay for private lessons at the Goethe-Institut, community colleges or private tutoring. Deutsche Welle-TV, the German news TV station, is only available from cable TV subscription. Newspapers printed in German and magazines are available only in selected newsstands and bookstores in the central business area. German films are occasionally available at selected 
cinemas and during film festivals. In other words, German is not part of the general linguistic landscapes of Hong Kong.

The Hong Kong school is matched with a secondary school in Berlin. The Berlin school offers Mandarin Chinese as an additional option. The project team included eight secondary school students and three English undergraduates on the Hong Kong side, eleven secondary school students and three pre-service English teachers on the Berlin side. The Hong Kong participants had been learning German for three years in an afterschool programme and had classes twice a week. After three years of learning German, some of the participants had already decided to drop the course at the end of the year. Some had decided to continue in the following year, and prepare for the British IGCSE German Examination. The project activities included workshops, a survey, blogging, video conferencing, self-portrait drawing, and focus group interviewing. Given the diverse data sources from both Hong Kong and German participants, this chapter focuses on the ways Hong Kong secondary school students see themselves as German learners in online and offline environments. The findings are drawn from data collected from a survey, from blogging, self-portrait drawing and focus group interviewing (table 1). All participants attended two workshops, and during the first workshop students completed a survey on their preferred German learning strategies and the potentials of using German popular cultural texts when learning German. In the project, each participant set up a personal blog and uploaded three to four entries over a 6-week period. The entries were written in English or German. Before the second video conferencing session, all participants drew their self-portraits as English and German learners. At the end of the project, participants discussed the challenges of learning German as a third language in Hong Kong. The interview was conducted in a mix of Cantonese and English and was then was transcribed and translated into English.

Table 1: Activities conducted for the Hong Kong participants

\begin{tabular}{|l|l|}
\hline Workshop 1 & Survey, set up personal homepages and blogs \\
\hline Blogging & $\begin{array}{l}\text { Entry 1: My hobby (English), } \\
\text { Entry 2: My neighbourhood (English/German) }\end{array}$ \\
\hline Video conference 1 & Video conferencing in one group \\
\hline Workshop 2 & Self-portrait drawing \\
\hline Blogs & Entry 3: Language learning stories (German) \\
\hline Video conference 2 & Video conferencing in three sub-groups \\
\hline
\end{tabular}

The focus of the analysis was to gain a general picture of the process of learning German as a third language and the ways participants positioned themselves in online and offline environments. Thus, a qualitative approach was adopted to 
create the narratives of learning. The data analysis focused on the 'subject reality', in other words, on "findings on how 'things' or events were experienced by the respondents" (Pavlenko 2007: 165). This is a dominant position in narrative inquiry that pays attention to what was told by the participants and I did not try to establish that what was told was an objective or accurate truth (Barkhuizen et al. 2013; Riessman 2008). Content analysis and open coding was used to extract themes from the oral and written narratives (Lieblich et al. 1998), and the themes were discussed with the participants in the focus group interview at the end of the project.

\section{$5 \quad$ Findings and Discussion}

During the first workshop, all participants (HKS-8) took a survey on their digital habits for English and German language learning. All participants were avid users of digital tools and social media (e. g. Facebook, MSN, and YouTube), and they were no strangers to using the English interface on these websites or applications. All participants also reported that they regularly consume English print and media texts like novels, TV programmes, popular music and video games. Participants all agreed that they found using non-text book materials to learn English is more enjoyable and relevant to their daily lives. However, their personal habit for learning German painted a very different picture. Over the years, participants regularly took German classes after school and on Saturdays. The language course was provided by the Language Centre of a nearby university, and focused on helping beginner learners to acquire German. Participants used a German learning textbook with some supplementary materials provided by the teachers. Participants did not appear to devote additional energy and time to searching for German print and media texts (Table 2). The reading materials were a couple German magazines distributed by the teacher. The biggest surprise was the absence of German popular music. While the participants were experts in English pop, they appeared to have very limited (or no) knowledge of German pop music. Among the participants, one (HKS2) never made any attempt to look for additional materials for learning German.

Table 2: Activities that participants had done prior to the project

\begin{tabular}{|c|c|c|c|c|c|}
\hline $\begin{array}{c}\text { Reading } \\
\text { German } \\
\text { comics/ } \\
\text { books/ } \\
\text { magazines }\end{array}$ & $\begin{array}{c}\text { Watching } \\
\text { German TV } \\
\text { programs or } \\
\text { movies }\end{array}$ & $\begin{array}{c}\text { Listening to } \\
\text { German } \\
\text { songs }\end{array}$ & $\begin{array}{l}\text { Visiting } \\
\text { websites in } \\
\text { German }\end{array}$ & $\begin{array}{l}\text { Playing } \\
\text { video games } \\
\text { in German }\end{array}$ & $\begin{array}{l}\text { Using German } \\
\text { in e-mail/ } \\
\text { online } \\
\text { messengers/ } \\
\text { SMS }\end{array}$ \\
\hline $\begin{array}{l}\text { HKS1, } \\
\text { HKS7, } \\
\text { HKS8 }\end{array}$ & HKS6, HKS4 & None & $\begin{array}{l}\text { HKS5, } \\
\text { HKS6, } \\
\text { HKS3, HKS4 }\end{array}$ & None & None \\
\hline
\end{tabular}


Another question addressing participants' preference of popular cultural text use in the classroom also revealed interesting results (Table 3). All participants considered films to be the best popular cultural texts for learning German. They were not particularly keen on using digital tools, like video games or websites. The participants preferred films because it was possible to watch German films with English subtitles, so they did not have to worry too much about using a bilingual dictionary all the time.

Table 3: Popular cultural texts preferred by participants (the number at the end indicated participants' priority).

\begin{tabular}{|c|l|l|l|l|l|l|}
\hline Comics & \multicolumn{1}{|c|}{$\begin{array}{c}\text { TV } \\
\text { programs }\end{array}$} & \multicolumn{1}{|c|}{ Movies } & Songs & Animations & $\begin{array}{c}\text { Video } \\
\text { games }\end{array}$ & \multicolumn{1}{|c|}{ Websites } \\
\hline HKS1-2 & HKS3-1 & HKS1-1 & HKS5-1 & HKS2-3 & HKS6-1 & HKS1-3 \\
HKS3-3 & HKS2-2 & HKS2-1 & HKS4-3 & HKS2-3 & HKS4-2 & \\
& HKS5-2 & HKS4-1 & HKS7-3 & HKS8-3 & & \\
& HKS6-2 & HKS7-1 & & & & \\
& HKS7-2 & HKS8-1 & & & & \\
& HKS8-2 & HKS3-2 & & & & \\
& & HKS5-3 & & & & \\
\hline
\end{tabular}

The participants also liked the idea of using film and TV presentations as an inclass activity to replace the regular listening and fill-in-the-blank activities. In the last question on how popular cultural texts help when learning German, almost all stated that these texts could integrate German into their daily lives.

One explicit point that the participants voiced during the project was the disconnectedness of German from their daily lives. Even when some participants did not mention this explicitly, their drawings of self-portraits as German learners illuminated the psychological distance. Participants were asked to draw themselves as learners of German on an A4 paper and write a short explanation for the drawing. This exercise was modeled after Kalaja et al. (2008) on using self-portraits among Finnish pre-service teachers. In the selfportraits produced by the students in Hong Kong, the most striking commonality was that almost all participants drew only one single character in their selfportraits, either a human figure or a metaphor. Four participants drew human figures (HKS1, HKS5, HKS6, and HKS8) while the other four (HKS2, HKS3, HKS4 and HKS7) drew metaphorical figures (e. g. bird, fish, insect, and water tank). In the human-figure drawings, none of the figures was holding anything. The figure drawn by HKS8 was the most telling (figure 1). The girl in the drawing was visibly unhappy, and she was holding a very stiff pose. The girl was also wearing her school uniform because learning German "happened in school and it was difficult" (HKS8). Other participants also drew their figures 
wearing school uniforms (rather than casual clothing) as an indicator of the place that for them was linked to learning German: school.

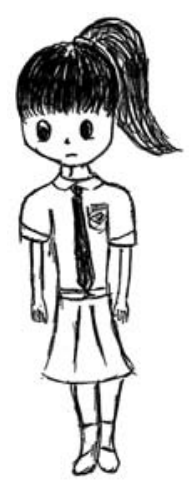

Figure 1: An unhappy looking HKS8

Participants who drew non-human figures did so based on the themes of "working hard" and "slowness" (figure 2). The picture of a caterpillar eating a leave was drawn by HKS7. She felt that her learning process was slow as the walking pace of a caterpillar but "[she] hope[d] that one day, [she could] turn into a butterfly which indicates I can learn German very well!" (HKS7). Other participants shared the same sentiment that they were not progressing fast enough. Unlike HKS8, participants who drew their self-portraits as an insect or a bird or a fish were not necessarily unhappy. In fact, from figure 2 , it was clear that the participant projected a better future for her process of learning German.

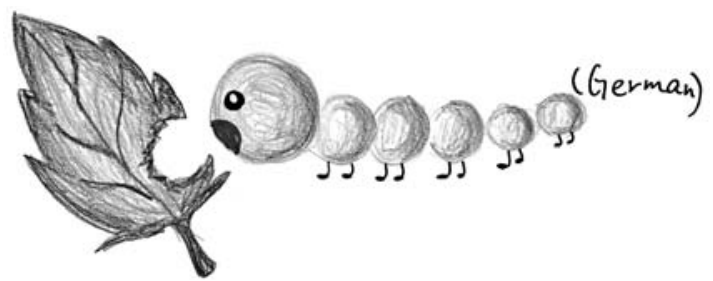

Figure 2: HKS7 drew her self-portrait as a hungry caterpillar

The participants in general indicated that their experiences while learning German had been challenging because the learning was limited to the classroom. They also did not find strong reasons to extend learning to their personal lives, because they were not familiar with German popular culture. When we did the call for participation, the eight participants were enthusiastic because this was a good chance to make some friends in Germany. For the project, we invited both the participants from Hong Kong and those from Berlin to blog about their hob- 
bies, neighbourhood and what they did to learn English, German or Chinese. During the first workshop, participants set up their personal homepages and blogs. On their homepages, participants were asked to write a short selfintroduction in either English or German. Among the eight participants, only two chose to write in German (HKS1 and HKS2). This was a breakthrough, as HKS2 was the only student who had no exposure to German media products in the month prior to the project, yet she chose to start a blog only in German. Some students reported that they were worried that their German was not "good enough" and the students from Berlin would not "understand [their] broken German" (HKS3). At the beginning, they were also shy to upload photographs of their own because "[they did] not know if the Berlin students want[ed] to see [their] face" (HKS3). After the first round of checking out the websites of the students from Berlin, those from Hong Kong were happy to see "real faces" (HKS4). It was at this point that the concepts of communicating with "real teenagers in German" kicked in, even though one could argue that the whole project was artificially constructed. The issue of authenticity can be further discussed in a different paper, but the motivation factor of communicating for what the participants perceived as an "authentic purpose" is more important.

The participants from Hong Kong were free to choose the language of blogging, and the research team encouraged everyone to blog in their target language. Three participants (HKS1, HKS2, and HKS3) started blogging in German from the first entry on their hobbies. Others wrote two blog entries in German on their neighbourhood and language learning strategies beyond the classroom (figure 3; cf. next page). 


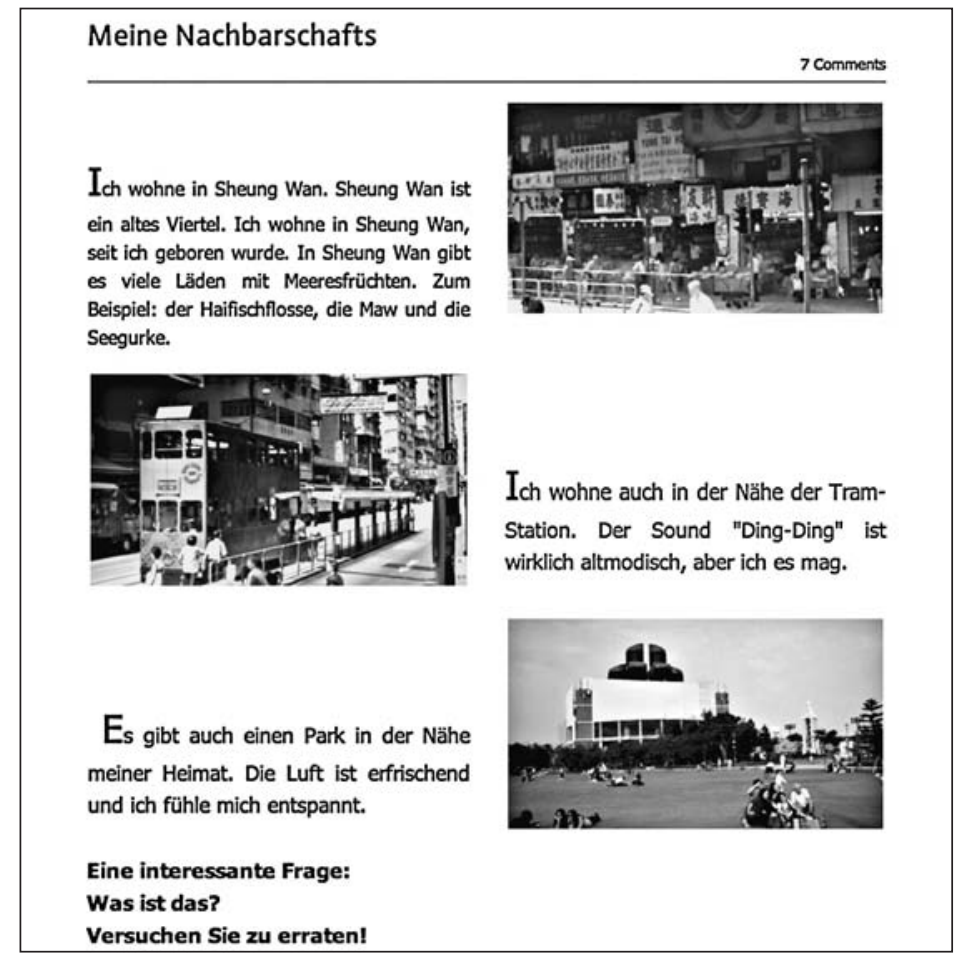

Figure 3: The blog entry on 'My Neighbourhood' by HKS1

The second journal entry on introducing a neighbourhood was an intersection between personal and academic purposes. During the focus group interview, participants mentioned that they did not think much about the neighbourhood they grew up in, and thought they had to think quite hard to say something about it. In addition, the requirement for visual elements made the task quite challenging because they wanted to show their German partners something unique about Hong Kong. They were motivated since some Berlin partners left them German comments, and they said they did not expect to feel happy about receiving comments, but they did. Writing in German to an authentic audience was something they thought they could not achieve in the classroom, because they had been completing writing tasks for their German teacher and the teacher alone. The task of writing was important because it allowed participants to produce German at their own pace. It was a "safe" exercise because "[They could] think about what [they] want to say and get some help from the dictionary" (HKS2). 
One observable point is that the Hong Kong participants were happy to put phrases like "*Please tell me my mistakes, I would like to correct it! Thanks!" (HKS2) into their blogs. This was quite different from their previous statements that underscored their need to "learn" German in the classroom. When asked about her code-switching in German and English, HKS2 was smiling and claimed that everyone on the project knew both languages. So it was natural that if she couldn't find the German phrases to express herself, she could codeswitch and no one would find it strange. In their online modes, these Hong Kong students were happy to ask for help and guidance in their writing. Many of them were also more confident in expressing themselves in writing. During the interview, they agreed that reading and writing activities were the "safer" activities and that they were happy to participate because they could complete the tasks in their own paces without worrying about making mistakes. The use of a blog was considered an ideal situation because they could now use photographs and possibly later find some German songs on YouTube to share with others.

Participants pointed out that they were less afraid of making mistakes than they normally were in their classroom writing, because trying to tell the Berlin students about their stories (for instance, their neighbourhood) was more important than getting the grammar right. But an interesting aspect raised by HKS7 pointed to the self-monitoring in blogging, "[she had been] more careful in [her] writing because [she had] wanted to express [her] ideas clearly, so [she had] checked the short passage several times before uploading it". All participants found that they had to use the dictionary more frequently because the new blog topics challenged their needs to adjust the tone and vocabulary. The challenges to write to an unfamiliar yet enthusiastic audience became "a thrilling experience" (HKS8) (figure 4; cf. next page). 


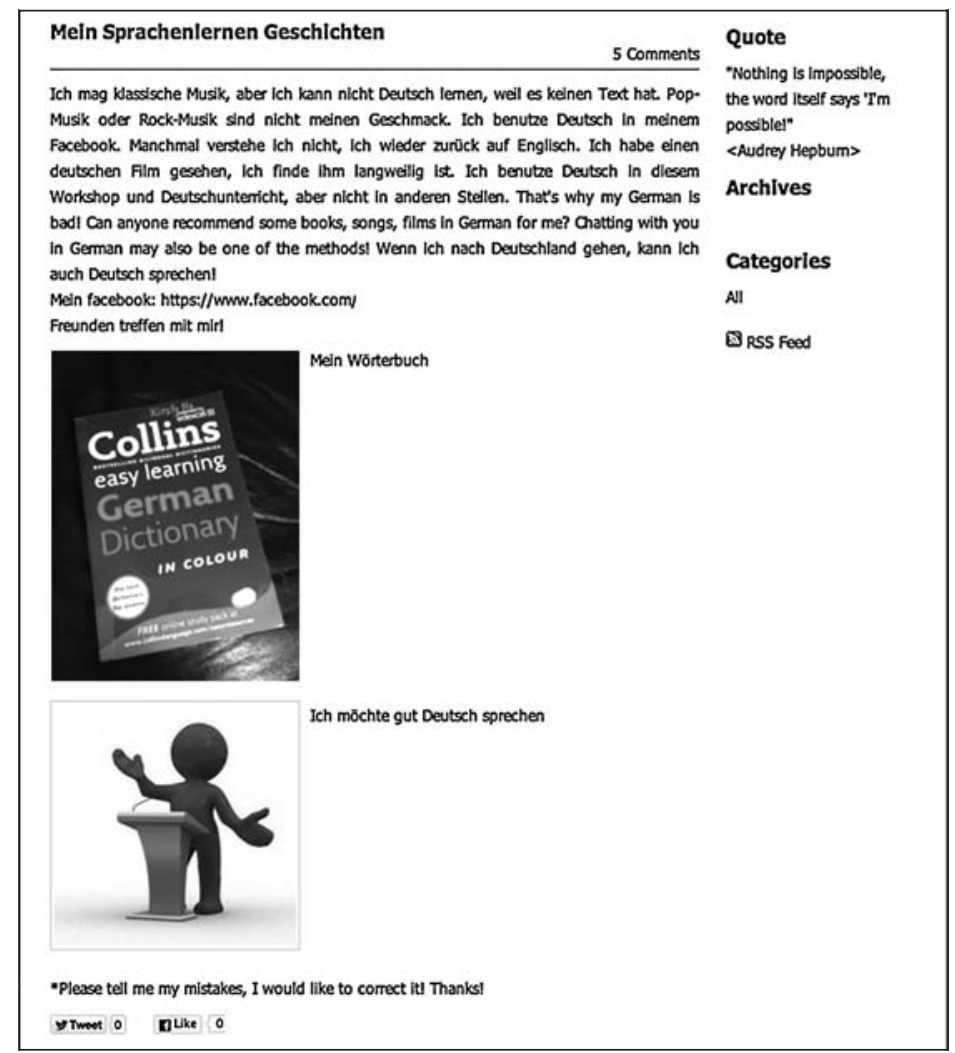

Figure 4: A blog entry on 'Learning a language' by HKS2.

Though McLaughlin (1990) pointed out that multilingual learners use different strategies to learn, the participants from Hong Kong appeared to be quite conservative at first in limiting their learning of German mainly to the classroom. It could be viewed that when the participants did not have a working knowledge of German popular culture, they found it difficult, if not impossible, to find media texts of personal interest as additional learning materials. Some tried to search for German pop singers, but were then frustrated by the random songs they found on YouTube. Some commented that the German films they went to see were "very serious" (HKS5). These teenagers were struggling to find the 'right' popular culture, and felt that they couldn't achieve it on their own. The self-portraits drawn by the participants also confirmed the ways these learners of German differentiated the locales of learning English and German, respectively: everyday practices to learn English but classroom-based practices for learning German. The challenge to find entry points to access German media 
texts was certainly not rendered easier by their varying levels of proficiency. The finding aligned with Csizér/Kormos (2008) that access to foreign language media texts enhances linguistic self-confidence. When faced with the daunting task of searching for media texts, many of the participants opted to give up. However, the participants' engagement with the online exchange showed that they were not indifferent to enjoying pop culture and digital practices in German, it was simply that they have yet to discover texts and practices of interest.

\section{Conclusions}

The present study confirmed that limited access to foreign language media texts could deter learners from fully engaging in and enjoying their L3 learning. Research on New Literacies Studies shows that SFL learners are capable of organizing and managing their SFL learning through different leisure and interest-driven digital practices, and these practices yield satisfactory learning experiences. The issue here with L3 learning is how to help learners transfer their skills of L2 digital practices to L3 digital practices. In the Asian contexts, an L3 is typically less common and less frequently used in sociocultural contexts, and thus an L3 student is essentially isolated except from his or her formal learning environment. In this case, the learners from Hong Kong have almost no or very limited exposure to German in their natural environments, for instance, at home or in school or in leisure activities. They could be viewed as 'underprivileged' in their learning of German. This is similar to many adults who are learning a foreign language for recreational purposes. For instance, for an adult Hong Kong learner studying French in Hong Kong, or a Japanese learner studying Italian in Japan, the best-case scenario that would see them surrounded by French or Italian in a non-threatening situation could well be in a French or Italian restaurant. Of course, this could be taking the argument to an extreme, but in linguistically poor environments, the challenges for modern language teachers would have to be helping learners to recreate a linguistically rich and sustainable environment for their learning process. Of course it may not be easy for L3 learners to have access to speech communities of their target language in their immediate social networks. L3 learners may still be able to construct media- and language-rich environments in order to enhance their learning progress.

In this article, we have come to understand young L3 learners' need for popular cultural texts in their L3. We have also seen practices to bring L3 learning and use into their social worlds; however, they need additional help and skills to achieve this goal. The responsibility of modern language teachers then may well be to help learners to anchor learning in foreign language media products and artefacts. 


\section{References}

Alvermann, Donna E. / Eakle, A. Jonathan (2007). Dissolving learning boundaries: The doing, re-doing, and undoing of school. In: Dennis Thiessen / Alison Cook-Sather (eds.): International Handbook of Student Experience in Elementary and Secondary School. Dordrecht: Springer. 143-166.

Barkhuizen, Gary / Benson, Phil / Chik, Alice (2013). Narrative Inquiry in Language Teaching and Learning Research. London: Routledge.

Black, Rebecca W. (2007). Digital design: English language learners and reader reviews in adolescent fan fiction. In: Knobel, Michele / Lankshear, Colin (eds.): A New Literacies Sampler. New York: Lang. 115-136.

Csizér, Kata / Kormos, Judit (2008). The relationship of intercultural contact and language learning motivation among Hungarian students of English and German. Journal of Multilingual and Multicultural Development 29/1: 30-48.

Csizér, Kata / Kormos, Judit (2009). Modelling the role of inter-cultural contact in the motivation of learning English as a foreign language. Applied Linguistics 30/2: 166-185.

Dörnyei, Zoltan / Csizér, Kata (2005). The effects of intercultural contact and tourism on language attitudes and language learning motivation. Journal of Language and Social Psychology 24/1: 1-31.

Falk, Ylva / Bardel, Camilla (2010). The study of the role of the background languages in third language acquisition. The state of the art. International Review of Applied Linguistics in Language Teaching 48/2-3: 185-219.

Federal Foreign Office (2012). Hong Kong. Online: http://www. auswaertiges-amt.de/EN/ Aussenpolitik/Laender/Laenderinfos/01-Nodes/Hongkong_node.html (last retrieval [10.07.2013])

Hammarberg, Björn (2010). The languages of the multilingual: Some conceptual and terminological issues. IRAL 48: 91-104.

Humphreys, Gillian / Spratt, Mary (2008). Many languages, many motivations: A study of Hong Kong students' motivation to learn different target languages. System 36/4: 313- 335.

Jessner, Ulrike (2008). Teaching third languages: Findings, trends and challenges. Language Teaching 41/1: 15-56.

McLaughlin, Barry (1990). The relationship between first and second languages: Language proficiency and language aptitude. In: Harley, Bridget / Allen, Patrick / Cummins, Jim / Swain, Merrill (eds.): The Development of Second Language Proficiency. Cambridge: Cambridge University Press. 158-174.

Kuure, Leena (2011). Places for learning: Technology-mediated language learning practices beyond the classroom. In: Benson, Phil / Reinders, Hayo (eds.): Beyond the language classroom. Basingstoke: Palgrave Macmillan. 35-46.

Lamy, Marie-Noëlle / Hampel, Regine (2007). Online Communication in Language Learning and Teaching. Basingstoke, UK: Palgrave Macmillan.

Lee, Carmen / Barton, David (2009). English and global identities on Web 2.0: The case of Flickr.com. In: Tam, Kwok-Kan (ed.): Englishization in Asia: Language and Cultural Issues. Hong Kong: Open University of Hong Kong Press. 1-31. 
Leppänen, Sirpa / Pitkänen-Huhta, Anne / Piirainen-Marsh, Arja / Nikula, Tarja / Peuronen, Saija (2009). Young people's translocal new media uses: A multiperspective analysis of language choice and heteroglossia. Journal of Computer-Mediated Communication 14/4: 1080-1107.

Lieblich, Amia / Tuval-Mashiach, Rivka / Ziber, Tamar (1998). Narrative Research: Reading, Analysis and Interpretation. Thousand Oaks, CA: Sage.

Nunan, David (2003). The impact of English as a global language on educational policies and practices in the Asia-Pacific region. TESOL Quarterly 37/4: 589-613.

O’Brien, David (2006). "Struggling” adolescents' engagement in mutlimediating: Countering the institutional construction of incompetence. In: Alvermann, Donna E. /. Hinchman, Kathleen A. / Moore, David W. / Phelps, Stephen F. / Waff, Diane R. (eds.): Reconceptualizing the Literacies in Adolescents' Lives. 2nd edition. Mahwah, NJ: Lawrence Erlbaum. 29-46.

Pavlenko, Aneta (2007). Autobiographic narratives as data in applied linguistics. Applied Linguistics 28/2: 163-188.

Poon, Anita Yuk Kang (2010). Language use, language policy and planning in Hong Kong. Polity Studies Series of Current Issues in Language Planning 11/1: 1-66.

Riessman, Catherine Kohler (2008). Narrative Methods for the Human Sciences. Los Angeles, CA: Sage.

Sheehy, Margaret (2009). Can the literacy practices in an after-school programme be practised in school? A study of literacies from a spatial perspective. Pedagogy, Culture, \& Society 17/2: 141-160.

Thorne, Steven L. / Black, Rebecca W. / Sykes, Julie L. (2009). Second language use, socialization, and learning in Internet interest communities and online gaming. The Modern Language Journal 93: 802-821. 



\section{Multilingual Virtual Talking Books (MuViT) - A Project to Foster Multilingualism, Language Awareness, and Media Competency}

\section{Daniela Elsner (Frankfurt/Main)}

This paper introduces the theoretical framework and the main objectives of the European Comenius project MuViT (Multilingual Virtual Talking Books). The project engages primary pupils across the world in multilingual and audiovisual reading and writing processes through the use of information technology resources. MuViT contains software with five digital storybooks in five different languages (English, German, Russian, Spanish, and Turkish), an authoring tool for story production and broadcasting, and a web community. The paper first sketches the necessity of new and innovative learning and teaching methods in line with a digitalized and multilingual society. In a second step it details on the objectives and tools developed within the MuViT project. Thirdly it illustrates which research questions and activities are connected with the project. First findings from exploratory research in Germany and in Turkey concerning the use of the program show the potential of the MuViT tools for multilingual and media education.

Der Beitrag erläutert den theoretischen Hintergrund und die angestrebten Ziele des EU Comenius Projektes MuViT (Multilingual Virtual Talking Books). Das Projekt fördert die Mehrsprachigkeit und Medienkompetenz europäischer Sprachenlerner in der Grundschule durch den Einsatz von mehrsprachigen, digitalisierten Bilderbüchern. Im Rahmen des Projektes wurden fünf Geschichten für den Computer in fünf Sprachen (Deutsch, Englisch, Russisch, Spanisch, Türkisch) entworfen, eine Authoring Tool zum Selbsterstellen und Publizieren eigener Geschichten sowie die MuViTWeb Community kreiert. Im ersten Teil des Aufsatzes wird die Notwendigkeit neuer und innovativer Lehr- und Lernformen im Kontext einer digitalisierten und mehrsprachigen Gesellschaft aufgezeigt. Im zweiten Teil des Beitrags werden die Ziele und Materialien beschrieben, die im Zentrum des Projektes stehen. Abschließend wird aufgezeigt, welche Forschungsfragen und -aktivitäten mit dem länderübergreifenden Projekt verbunden sind. Erste Ergebnisse aus explorativen Studien in Deutschland und in der Türkei weisen auf ein hohes Potenzial der Materialien und deren Verwendung im Unterricht im Hinblick auf die Entwicklung von Mehrsprachigkeit und Medienkompetenz hin.

\section{Changes in our society - changes in our classrooms}

As a consequence of migration, global economic expansion, and increasing mobility, our society can be characterised by growing cultural and linguistic diversity. The Eurobarometer 2006 (European Commission 2006) states that 56 per cent of all EU-citizens speak at least one foreign language in addition to their mother tongue. 28 per cent indicate that they even master two foreign languages apart from their first language. Many internet websites can be 
optionally chosen and read in different languages, besides many of them making use of a mixture of languages on one and the same page, to present different products internationally or simply illustrate content in a 'multicultural' and thus open-minded manner. A view into common YouTube blogs shows how people today, being at different places all over the world, mediate from one language to another when discussing a video clip or a song, or when explaining to others difficult aspects of online tutorials. It becomes obvious that plurilingualism has turned from an exception to a norm.

Linguistic diversity goes hand in hand with the rapid development of new information technology, people all over the world communicate with each other, exchange information, read the same texts being in completely different places and speaking different languages. The new generation - the so called "digital natives" (Prensky 2001) are constantly connected with friends via smartphone, tablet or a computer, at any time of the day being at different locations in any country in the world. This generation cannot imagine anymore, how it was, when communication was still restricted to face-to-face or at least voice-to-voice situations, and what it meant for working processes when people exchanged or received information through printed texts only.

Most students today are more motivated to read multi-codal texts from a computer screen than mono-codal texts without images from paper in hand, and they feel far more comfortable when typing on a keyboard than writing in a spiral notebook. According to a recent statistic by the EU, more than 75 per cent of EU-citizens use a computer for communication and information exchange with other users on a regular basis - with this trend still growing (cf. Eurostat 2012).

These societal changes have also entered European classrooms, as different surveys stress the prevalence of children already at primary level using computers in a frequent mode. In addition, classrooms host children who bring along completely different linguistic preconditions, ranging from almost equally high levels of proficiency in two or more languages to deficient levels of competence in one or more languages spoken by the child. In Germany, for instance, many pupils often speak two mother languages other than German, such as Turkish and Kurdish. In most cases these children are not alphabetised in those languages and thus not able to read and write in their L1(s). When they enter kindergarten or school, these children do not possess a large lexicon in German in addition, as they have not gathered any literacy experiences in the language of their surrounding until this point, which often leads to grammatical difficulties in all of their available languages. Those children need extra support with regard to their L2 but also in their L1. In other cases children are proficient speakers in at least two languages and able to read and write in their available 
languages - as their parents speak with and read to them in both languages from very early on.

It is a commonplace that new concepts for learning and teaching are needed, that include and further develop pupils' different linguistic and technological qualifications.

\section{Consequences and demands}

Long ago educational policies reacted to the fact that our world has become linguistically and culturally diverse and that communication increasingly takes place in a multilingual way. On a European level, multilingualism is therefore a defined educational goal. In 2001, the European Year of Languages, the Council of Europe has called for the 'retention and promotion of multilingualism within the European Union' (Council of Europe 2001). In 2008 the European Commission declared multilingualism as "an asset for Europe and a shared commitment" (European Commission 2012: 3). The German 'Integrationsplan' calls on educators to see individual plurilingualism in our schools as a chance for the development of mutual understanding, tolerance and respect, and motivates teachers to incorporate different languages into their daily teaching routines (cf. Federal Ministry of Germany 2007).

In the same vein, foreign language pedagogy proclaims that "the potential of migration-induced plurilingualism [...] should be fully utilised" (Kollmeyer 2007: 258, translation D. E.) and that "linguistic diversity has to be regarded as an opportunity and not a task" (Luchtenberg 1997: 121, translation D.E.). Language acquisition research has confirmed that a successful integration of the mother tongue can facilitate second language learning for plurilingual children (cf. Caprez-Krompàk 2007; Gogolin 1988; Videsott 2011), and that an appreciation and integration of pupils' first languages supports pupils' identity formation in multilingual surroundings (cf. Cummins et al. 2005; Cummins 2001; Videsott 2011). Moreover, studies in the field of tertiary language learning suggest that pupils especially benefit from their plurilingualism with regard to learning new languages, when the learning environment affords them to make use of their available languages, e. g. by offering tasks that include a variety of languages (e.g. Gibson/Hufeisen 2007; Singleton/Aronin 2007). Hélot/Young (2002) demonstrate how, apart from the plurilinguals, even monolingual pupils may profit from such multilingual approaches in teaching and learning with regard to the development of language awareness. They not only learn more about different language systems but, by comparing these to their own, they learn more about their own mother tongue on top (cf. Hélot/Young 2002). 
As a consequence, several suggestions for the integration of pupils' family languages into first or foreign language classrooms and the interconnection between modern foreign languages have been made. However, most of these ideas are restricted to simple comparisons of the languages on the word or chunk level and are only suitable for pupils at secondary level. Only few suggestions consider an active integration of heritage languages at primary school level (cf. Elsner 2010; Esteve 2004; Preker-Franke/Preker 2011; Reif-Breitwieser 2004).

A definite lack of methodological guidelines for the application and implementation of multilingualism in primary schools and the unanswered question how to include other languages apart from the target language can be named as the most prominent reasons for the absence of other languages in the foreign and native language classroom (e. g. Kollmeyer 2007). Neither curricula nor teacher training or advanced teacher education programmes offer concrete practical suggestions or teaching materials for the realisation of this issue in language classrooms so far. An often rather deficient awareness of languages, coupled with insecurity in dealing with unknown languages, are essential reasons why, apart from the well-known and often criticised "monolingual habitus of the German school" (cf. Gogolin 1994), multilingualism is still ignored as a learning objective and a learning prerequisite in most classrooms to date. In a nutshell, even though most teachers strongly agree with the educational recommendations concerning a multilingual approach, they are not empowered to practically transfer this into their classrooms, since they have not been equipped with the appropriate knowledge, skills, and materials.

It is for the same reason that teachers are very hesitant when it comes to the integration of new media into their teaching and learning surroundings. More than 81 per cent of our pupils in the age between 8 and 16 use a computer at home on a daily basis, for playing games, learning, social interaction or information purposes (cf. IconKids \& Youth 2008), but only 33 per cent of our pupils use computers at school at least once a week (Medienpädagogischer Verbund Südwest 2010: 29). Many teachers admit that they do not feel sufficiently prepared for the integration and use of technology in their classrooms and thus hardly ever incorporate technology-based activities such as internet research, online-games, web-quests or edutainment software. Teachers feel that they need further training and/or explicit methodological guidelines for working with the new media in their specific classrooms (Grossmann 2008: 14; Wiedwald et al. 2007: 49). Not very surprisingly, blackboard and print media are still the media of teachers' first choice and the prime media to be worked with in schools. In contrast to this, pupils at home tend to gather multimodal experiences with digital texts on their computer, their smartphone or tablet. Many studies highlight how the attractive potential of new media is causing a threat to the young generation's functional literacy skills, with images, graphics and sounds substituting reading and writing as a means of transmission of 
information. Especially the chaotic flow of information can be misleading for pupils whose critical thinking skills have not yet been sufficiently trained (for further information about the use of media see http://www.onair.medmediaeducation.it/project.aspx, website of the European media education project OnAir).

Education policy makers all across Europe therefore see the necessity of media education from the early years on and are keen to integrate it as a crucial part of education in their schools. Yet, the development and implementation of clear structures for media education vary widely across Europe. "There seems to be a huge gap between those members who have been implementing media education for years, and those without any sustainable infrastructure for its integration and implementation in their schools." (Lemmen 2005: 1). While the UK, the Netherlands, and some of the new member states such as Hungary provide good examples of media education, other countries, including Germany, have just started to integrate the development of media competence as one crosssubject objective into their regional curricula. Nonetheless, teachers are rather left alone when it comes to concrete examples of good practice. In consequence, this often leads to ignorance or refuse of technical devices in the classroom.

The European Comenius project MuViT has been initiated based on this set of problems and needs. It seeks to combine both aspects elaborated on: The integration of many languages into our classrooms in combination with the use of new media. As UNESCO (2011) points out: "New information and communication technologies (ICT) can serve not only to encourage linguistic diversity and multilingual education but also to raise awareness of linguistic and cultural traditions throughout the world and to inspire solidarity based on mutual understanding, tolerance and dialogue."

MuViT is designed to animate primary school pupils to read, write, compare, and work with texts in different languages on the computer. The following chapter will depict the project and its objectives.

\section{The European Comenius Project MuViT}

\subsection{Project Objectives}

The project name MuViT stands for Multiliteracy Virtual, pointing out the central ideology of the project which can be summarized as follows: Language learning should emerge from the cultural, linguistic, and digital experiences that learners bring into our classrooms and aim at the further development of a broad range and new forms of literacies, including functional, visual and multimodal literacies, media competencies, plurilingual awareness and critical thinking skills, altogether understood as multiliteracies (e. g. Elsner 2012). With this in 
mind, the MuViT team-members, being researchers in the field of first, second and/or foreign language acquisition, teacher educators, teachers, or ITspecialists from Germany, Spain, Turkey, Latvia, and Russia, searched for a simple-to-use tool which could put this multiliterate notion into practice by enhancing multilingual learning and likewise the development of language awareness through the use of new media. Since a start in January 2011, the goal has been to develop, implement, and evaluate a concept for the use of digitalised, multilingual talking books that allow learners to simultaneously and autonomously work in their first, their second and a modern foreign language on the computer screen (MuVit player), alongside an authoring tool enabling children to produce and share their own multilingual storybooks within the MuViT web-community.

\subsection{MuViT products in detail}

The MuViT player consists of six age-appropriate stories in English, German, Russian (Latin and Cyrillic alphabet), Spanish and Turkish. The illustrated stories are read out by native speakers and a simultaneous highlighting function (similar to karaoke) is available in which words of the written story are marked at the moment they are spoken in order to support text comprehension.

Two pages of vocabulary introduction precede each story. On the first page key-words of a story are displayed within a context in order to facilitate the understanding of the story. The second page repeats important vocabulary separately, helping pupils to memorize and pronounce important key words. On both pages, as well as on the following pages, children can switch between languages at all times.

Subsequent to the stories in multiple languages, tasks related to text comprehension, language awareness, cross-linguistic comparisons, and language reflection follow. With these, pupils are supposed to be sensitised for different languages by doing cross-linguistic comparisons and to develop conscious awareness of rules in their available, targeted and chosen languages. Following the tasks the pupils are asked with which languages they have dealt within the software. Moreover, they are supposed to grade the difficulty of the stories and tasks in different languages. With the help of application protocols automatically recorded by the software, teachers are given the opportunity to gain insight into their pupils' ability to handle the different languages and, if necessary, provide an impetus to work more intensively on one or the other language or try out new languages. The software is primarily designed for primary school pupils, however, the stories are also suitable for the transition to secondary level.

The additionally developed Authoring Tool allows children from all over the world to write, record, and design their own stories for publication. The tool 
enables all learners to upload their stories, accompanying images and audio files. These stories can be exchanged with others, translated, and distributed within the MuViT Web Community, a registration system and forum for pupils and teachers all over the world.

A teacher handbook and materials for in- and pre-service teacher training workshops (cf. Wildemann et al. in this volume) have been developed in order to provide methodological guidelines for the use of the MuViT products in the classroom. All of the materials, including the software, tutorials for using the software and the authoring tool and teacher-training handbooks can be found on the MuViT website: www.muvit.eu.

\section{Research in the context of the MuViT project}

The central objective of the MuViT project is to foster the development of multilingualism with a special focus on language awareness and multilingual identity formation. Primary level pupils shall deal with their own and other languages in order to gain a basic or initial knowledge of structures and inventories of different languages. At the same time MuViT is supposed to arouse pupils' interest in and curiosity of other languages. Moreover, dealing with numerous languages and obtaining multilingual awareness will improve the status of minority languages in the classroom, too. The MuViT software encourages the user to actively code-switch which is already a part of plurilingual pupils' existing daily discourse-behaviour. In addition, the work with the digital texts is supposed to support pupils in the development of functional literacies - understood as reading and writing skills - in all of their spoken or learned languages. Besides these language-oriented goals, MuViT intends to challenge pupils to explore and use the media application (software, authoring tool and community) self-determinedly. MuViT therefore contributes to the development of media competencies as described by Vollbrecht (2001): Students should become able to apply media as a means of communication.

\subsection{Exploratory Research during the project lifetime}

Within the funded period of the project (1/2011-12/2012) there had been little time for research investigating whether the MuViT products and their implementation in schools can fulfill the targeted aims. However, our project group started with some exploratory research in all of the participating countries using different research methods, such as questionnaires, interviews and observations in order to gather first information on pupils' exposure to the software and their overall perception. 


\subsubsection{Research questions}

The observations and interviews should primarily fathom the potential of the software and give an answer to the following questions:

- Do mono- and plurilingual pupils make use of the different languages offered in the software?

- How often and why do pupils switch languages?

- What do pupils talk about when using the software in pairs?

- How do pupils like the MuViT programme overall?

- How easy/difficult/motivating do they find the handling of the medium?

\subsubsection{Research design}

The first inquiries with the software were conducted in spring of 2012 based on a pre-release version of the prototype MuViT 1.0. Subject groups were 69 fourth grade pupils from four primary schools in Frankfurt, Germany and Istanbul, Turkey. Both, the German and the Turkish group, are similar due to the high level of multilingualism in all classrooms. In opposition to the Frankfurt classes with their several different language combinations (Russian-German, ArabicGerman, Serbo-Croatian-German), the group from Istanbul was more homogenous since the majority of this class is growing up bilingually with German and Turkish. Both classes also included monolingual German or Turkish children. MuViT could be deployed in the Frankfurt classes and pupils were observed and partially filmed while working with the software in class. Observations concentrated on code-switching behaviour of the pupils (How often and when?) and their behaviour while solving the tasks (Can they solve the tasks? Do they need help? Do they make use of different languages?), as well as during working with the software (Is the software self-explaining or do pupils need help?). Selected pupils were then interviewed using a semi-structured interview guideline that focused on pupils' experiences and asked for their overall perception of the programme (How did you like the work with the programme? Was it easy or difficult? How many stories did you read? How did you choose them? What did you like/didn't you like? Is there anything you would like to improve?) and their reasons for choosing and switching languages. In another classroom in Frankfurt and in a school in Istanbul pupils were asked to work with MuViT in pairs. Their interaction was recorded and analysed with regard to the question if their communication showed evidence of language awareness. Questionnaires were given out to the pupils in order to determine pupils' language background and their attitude towards their own and other languages. 


\subsubsection{First Results}

Naturally, we are aware that the data generated within the exploratory research study cannot deliver any generalisable results but it allows for construction of hypotheses (e. g. Kleemann/Krähnke/Matuschek 2009).

\section{MuVit arouses interest and curiosity for different languages}

Clearly positive is the pupils' estimation that the MuViT software has aroused their interest and curiosity for other languages. All of the observed and interviewed pupils confirmed that they liked working with the software and that they would appreciate to work with this or similar materials in the classroom in future. In dealing with the software it was observed that the interest in what the pupils described as "exotic" languages was particularly high. For instance, German and Turkish pupils liked listening to (passages of) a story in Russian so as to experience "how Russian actually sounds because I have never heard Russian before". They also mentioned that they would welcome the integration of other languages than those already offered in the language-menu. They listed such languages as Chinese, Japanese, or French. Bearing in mind that those are "important" (Chinese) and "beautiful" (French) languages they expressed the wish to consciously listen to and learn them. When asked what she would do with the software if she had more time, a German-Turkish girl replied that she would pick a Russian sentence from a story and listen to it so often that she would learn it by heart. This way, she explained, "I would be able to speak a real Russian sentence and that would be very cool". Generally speaking, the listening experiences enabled by the software seem to hold a huge fascination.

\section{MuVit contributes to the development of language awareness and identity formation}

When pupils were working with the software, it was interesting to observe that the mother tongue or the dominant language for bilingual children, respectively (usually German, being the language of instruction at all schools) is used as the major reference language. It serves as a reassurance to check if passages, sentences, or words were understood correctly. Comprehension backup, consequently, is a common occasion for code-switching. It is not surprising that the reference language for bilingual pupils in Germany and in Turkey, e. g. Turkish-German children, is always German. This can be explained by the fact that there has not been a systematic alphabetisation of the second language in a school context and Turkish in the case of the Istanbul group is merely seen as a foreign language and will not become a medium of instruction. The children explained this by saying that "German is our better language because we know more words, read more in German und are able to write more." 
An entirely different picture could be drawn in interactive situations with the software when, for instance, a monolingual German-speaking pupil worked with a German-Turkish speaking child. The alleged advantage that a monolingual German-speaking pupil experiences in the classroom at school, a context in which plurilingualism is perceived as a problem and whose curricular premises and educational goals are based on monolingualism, makes way for a new experience: plurilingualism is perceived as an advantage in this setting. The bilingual girl realises that her knowledge in two languages is not a handicap here but helps her when dealing with the software. She is the one dominating and controlling the interaction thanks to her plurilingual competence, helping and giving advice to her monolingual German-speaking friend. After working with MuViT, a pupil at the Istanbul school stated that her stronger language is German, but added proudly: "By the way, I can also speak Turkish!" Throughout the questionnaire, prior to the testing, she had not mentioned her Turkish skills. By dealing with MuViT she has obviously experienced that her plurilingual knowledge is definitely to be valued as beneficial and that Turkish can be just as valuable as other languages.

It can be assumed that the above depicted situations have a positive effect on the development of language awareness on a "social-educational level" (e. g. Breidbach et al. 2011: 14). Future studies will deal not only with this aspect but also with the question of how identity formation of plurilingual pupils is influenced by working with MuVit.

In addition, it could be seen that pupils actively analyse the rules of the languages that they read the stories in. One monolingual German pair, for example, stops at a certain point in a story and talks about negation aspects in the English language (see Wahl 2012: 56):

Pupil 1: "Da ist schon wieder das t" ("There is the t again", translation D. E.)

Pupil 2: "Doesn't, he does not want to go to school. Right."

Without being asked to, pupils autonomously discuss grammatical aspects of a language, which in the long run, should contribute to the development of language awareness on a "linguistic-systematic level" (Breidbach et. al. 2011: $14)$.

Pupils consciously make use of different languages when working with MuViT 'Code-switching', as defined by Gumperz (1973), refers to the alternate use of two or more languages in the same utterance or conversation. It is the skill to switch between two or more languages due to communicative necessities during a conversation in a way that is appropriate for that particular situation. For children and teenagers with a so-called migration background, in particular, code-switching is a daily necessity and a useful strategy as it allows them to 
compensate linguistic deficits in one language by resorting to their knowledge in another language. On the other hand, this form of 'language contact' is very challenging and is based on creativity, linguistic knowledge, and language competence.

MuViT explicitly invites users to an unbiased approach to code-switching with its range of different languages. And the observations could show that all children, no matter if they are mono- or plurilingual, make use of the different languages. However, it could be seen that the plurilingual children switch more often between the different languages offered in the programme than the monolingual children. Comprehension difficulties or insecurities are frequently observed when switching to the reference language within a story for the purpose of making sure that the content is understood correctly (see Wahl 2012: 56):

Pupil 1: Also warte, warte, warte, warte. Also, erst mal sagt er, sagt von ihm die Mutter, 'willst du heute zu die Schule gehen?' Und er sagt 'ja'. Und die Mutter sagt, ähm, 'geh wie jeden Tag', also nein (reads), just like, 'ich liebe diesen Tag', sagt sie glaub ich.

Pupil 2: Dad, da steht 'Dad'. Seinen Papa, nicht den Tag.

Pupil 1: 'Day' ist Tag.

Pupil 2: Ach so.

Beyond comprehensive aspects, stylistic features prompt the young users - no matter if they are mono- or plurilingual - to switch to another language: The magic spell in the story 'Magic Maddox' ends with a rhyme ('Abracadabra, one and two, there is a goldfish in my shoe') whereupon two girls in Turkey checked this page of the story systematically in all other languages to find out whether the magic spell rhymes in all of them (it only does in Turkish!). A giant, ugly and dangerous looking dog, ironically named 'Sugarplum', plays a key role for the punch line of the story 'Mister Miller'. The funny surprise effect is a reason for these girls to check the name of the dog in all other languages.

Altogether, it can be concluded that comprehension insecurities as well as stylistic finesses such as funny names, exaggerations or contrasts (to the picture information) can act as stepping-stones for code-switching. In the pupils' reflections they serve for the purpose of language comparison, which suggests the assumption that $\mathrm{MuViT}$ - at least on a receptive level - in fact contributes to an unbiased practice of code-switching. Unlike in daily school situations, codeswitching can be used as a conscious strategy for language comparisons in the context of the software and subsequently make a valuable contribution to the development of language awareness. This assumption shall also be followed up in future studies. 
MuVit contributes to media education

The MuViT software was presumed to have a positive influence on the development of media competencies. However, our observations could show that the handling of the programme was no challenge to the pupils at all as they had no problems or questions when working with the programme. However, pupils clearly pointed out the deficits of the software, such as the lack of adaptability with regard to difficulty (choice of levels) or more language options. This shows that pupils are able to reflect on what media might be useful for. A higher learning potential is expected for the Authoring Tool where apart from handling the PC and software the development of presentation competencies and publishing skills play a vital role. Since the activation of this tool on the Internet platform has just started, this assumption will be a subjectmatter for future studies.

\section{Summary and further research}

The EU-funded Comenius-Project MuViT has been developed against the background of the two key changes in our society which strongly influence people's daily discourse and information behaviour. The MuViT tools have been invented to make a significant contribution to multilingual and media education in school. Based on first classroom observations and recorded interactional situations as well as first interviews with the target group of fourth graders in Turkey and Germany, preliminary insights into the application and benefits of the multilingual software have been gained, leading to various theses that need to be further explored. Supposedly, working with the software has a positive impact on the identity formation and the development of language awareness of mono- and plurilingual children alike. The integration of languages such as Turkish seem to be of particular value in immigrant societies such as Germany, in which the reputation of immigrant languages is rather poor among the majority of the population, because it is associated with a low social status.

As it enhances an unbiased dealing with the software and supports codemixing strategies, MuViT can help to equalise the linguistic relations in classrooms on the individual level of personal learning experiences. A larger research project, concerned with the question if MuViT fosters language awareness on the linguistic-systematic and the social-educational level, is currently being undertaken by Viviane Lohe, University of Frankfurt, who is working on her $\mathrm{PhD}$ with the title Developing Language Awareness in Primary School Children through Multilingual Virtual Talking Books.

The observations could also show that pupils read and listen to the texts with great interest and that they do this in different languages of their choice. It needs to be further researched how pupils' reading, writing, and listening skills in 
different languages may benefit from working with the programme and in how far the multimodal input (audio-visual and different languages) may be especially suitable for foreign language learners. Financing for a larger interdisciplinary project titled Wirksamkeit von multimodal-mehrsprachigem Input für das fremdsprachliche Textverstehen bei ein- und mehrsprachigen Schülerinnen und Schülern in der Grundschule (Effect of multimodal-multilingual input on foreign language text comprehension of mono- and plurilingual pupils at primary level) has just been applied for at the Ministry of Education in Germany. The project will start in August 2013 and will be conducted by three researchers from Frankfurt University, Daniela Elsner, Ilonca Hardy and Astrid Jurecka.

Furthermore, it will be interesting to see how pupils use their available languages when reading and listening to the stories in rather unknown or newly learned languages. Observations made in this context allow for the conclusion that most often the dominant language spoken at school serves as a reference language for the children in case of doubt and when comprehension problems occur. This also gives an important hint to the meaning of previously learnt languages for further language learning. Especially cooperative settings seem to trigger the use of different languages when working with the programme. However, which constellations and tasks are the most effective ones when using the software is a question that is being researched in a project funded by the Federal State of Hesse, Germany and undertaken by Daniela Elsner, Ilonca Hardy and Judith Bündgens-Kosten, all Frankfurt University, and titled Bedeutung der Erst- und Zweitsprache bei Lernern der Fremdsprache Englisch für die kooperative Bearbeitung textbasierter Lernaufgaben. (Meaning of L1 / L2 for learners of English as a foreign language during cooperative and textbased learning processes.)

Last but not least, MuVit does not only cater to pupils but also to teachers as it gives them the chance to learn more about their own and other languages, including some family languages of their pupils by using a computer programme. Raising awareness for the fact that there is a multilingual reality inside and outside the classroom and that, besides the dominant school language, other languages exist that are worth learning about. This might help to counteract the current dominance of the school language as the only language of education. If this were to be accomplished, a small but important step towards a more balanced relation of languages in a modern, multilingual, technologydriven, transmigration society would be taken. 


\section{References}

Breidbach, Stephan / Elsner, Daniela / Young, Andrea (2011). Language Awareness in teacher education: Cultural-political and socio-educational dimensions. In: Breidbach, Stephan / Elsner, Daniela / Young, Andrea (eds.) (2011): Language Awareness in teacher education. Cultural-political and socio-educational dimensions. Frankfurt/M.: Lang. 1122.

Caprez-Krompàk, Edina (2007). Die Bedeutung der Erstsprache im Integrationsprozess. terra cognita 10: 72-75.

Cummins, Jim (2001). Language, Power and Pedagogy: Bilingual Children in the Crossfire. Clevedon: Multilingual Matters.

Cummins, Jim / Bismilla, Vicki / Cohen, Chow / Patricia Sarah / Giampapa, Frances / Leoni, Lisa / Perminder, Sandhu / Sastri, Padma (2005). ELL Students Speak for Themselves: Identity Texts and Literacy Engagement in Multilingual Classrooms. Online: http://steinhardt.nyu.edu/scmsAdmin/media/users/ccm246/Haynes_2010/ELLidentityTexts.pdf__last retrieval [20.03.2013]).

Elsner, Daniela (2010). „Ich habe was, das du nicht hast...“ Oder: Welchen Mehrwert hat die Mehrsprachigkeit für das Fremdsprachenlernen. IMIS Beiträge 37/2010: 99-120.

Elsner, Daniela (2012). Developing Multiliteracies, Plurilingual Awareness \& Critical Thinking in the Primary Language Classroom with Multilingual Virtual Talking Books. Encuentro Journal 20/2011-12: 1-12.

Esteve, Olga (2004). Language Awareness im mehrsprachigen Kontext Kataloniens: Lernaktivitäten zum Aufbau eines ganzheitlichen Sprachlernkonzeptes. Fremdsprache Deutsch 31/4: 44-47.

European Commission (2006). Eurobaromenter Spezial: Die Europäer und ihre Sprachen. Zusammenfassung. (Eurobaromenter Spezial 243 / Welle 64.3 - TNS Opinion \& Social) Online: http://europa.eu.int/comm/public_opinion/archives/ebs/ebs_243_sum_de.pdf (last retrieval [20.03.2013]).

European Commission (2012). Key Data on Teaching Languages at School in Europe 2012. Brussels: EACEA.

Eurostat Press Office (2012). Computerkenntnisse in der EU27 in Zahlen: Pressemitteilung zur E-Skills Woche 2012. Online: http://epp.eurostat.ec.europa.eu/cache/ity_public/426032012-ap/de/4-26032012-ap-de.pdf (last retrieval [20.03.2013]).

Federal Ministry of Germany (2007). Der Nationale Integrationsplan 2007: Neue Wege Neue Chancen. Online: http://www.bundesregierung.de/Content/DE/Publikation/IB/Anlag en/nationalerintegrationsplan, property=publicationFile.pdf (last retrieval [20.03.2013]).

Gibson, Martha / Hufeisen, Britta (2007). Überlegungen zur Tertiärsprachenproblematk aus der Sicht der Psycholinguistik und der Fehleranalyse. Zeitschrift für Fremdsprachenforschung 18/1: 27-41.

Gogolin, Ingrid (1988). Erziehungsziel Zweisprachigkeit: Konturen eines sprachpädagogischen Konzepts für die multikulturelle Schule. Hamburg: Bergmann \& Helbig.

Gogolin, Ingrid (1994). Der monolinguale Habitus der multilingualen Schule. Münster: Waxmann. 
Grossmann, Schimon (2008). Supplementing Textbooks with Computer-Based Resources in the Primary EFL-Classroom. (unveröffentlichte Masterarbeit). Freiburg: Pädagogische Hochschule Freiburg.

Gumperz, John J. (1973). The communicative competence of bilinguals: Some hypotheses and suggestions for research. Language in Society 2/1: 143-154.

Hélot, Christine / Young, Andrea (2002). Bilingualism and Language Education in French Primary Schools: why and how should migrant languages be valued? International Journal of Bilingual Education and Bilingualism 5/2: 96-112.

IconKids \& Youth (2008). CHIP-Studie: Kids am Computer. Online: http://www.chip.de/ downloads/CHIP-Studie-quot-Kids-am-Computer-quot_30865353.html (last retrieval [20.03.2013]).

Kleemann, Frank / Krähnke, Uwe / Matuschek, Ingo (2009). Interpretative Sozialforschung. Wiesbaden: Verlag für Sozialwissenschaften.

Kollmeyer, Katrin (2007). Englischunterricht als Fenster zur Mehrsprachigkeit. In: Elsner, Daniela / Küster, Lutz / Viebrock, Britta (eds.): Fremdsprachenkompetenzen für ein wachsendes Europa: Das Leitziel «Multiliteralität». Frankfurt/M.: Lang. 257-268.

Lemmen, Ruth (2005). Europe. Media ed. Teach film, media and filmmaking. Online http://mediaed.org.uk/media-literacy/media-literacy-in-europe (last retrieval [20.03.2013]).

Luchtenberg, Sigrid (1997). Language Awareness: Anforderungen an Lehrkräfte und Probleme bei ihrer Ausbildung. Fremdsprachen Lehren und Lernen 26/1: 111-126.

Medienpädagogischer Forschungsverband Süd-West (2010). KIM-Studie 2010. Online: http://www.mpfs.de/fileadmin/KIMpdf10/KIM2010.pdf (last retrieval [20.03.2013]).

Prensky, Mark (2001). Digital Natives, Digital Immigrants. On the Horizon 9/5: 1-6. Online: http://www.marcprensky.com/writing/prensky $\% 20 \% 20$ digital $\% 20$ natives, $\% 20$ digital $\% 20 \mathrm{i}$ mmigrants\%20-\%20part1.pdf (last retrieval [20.03.2013]).

Preker-Franke, Annegret / Preker Philipp (2011). Englisch und Mehrsprachigkeit: Kopiervorlagen für die Klassen 8-10. Stuttgart: Klett.

Reif-Breitwieser, Susanne (2004). Wir sprechen zehn Sprachen: Bericht über ein multilinguales Sprachenprojekt in mehreren Teilen. Fremdsprache Deutsch 31/4: 30-25.

Singleton, David / Aronin, Larissa (2007). Multiple Language Learning in the Light of the Theory of Affordances. Innovation in Language Learning and Teaching 1/1: 83-96.

UNESCO (2011). Multilingualism on the internet. Online: http://www.unesco.org/new/index. php?id=50219 (last retrieval [20.03.2013]).

Videsott, Gerda (2011). Mehrsprachigkeit aus neurolinguistischer Sicht: Eine empirische Untersuchung zur Sprachverarbeitung viersprachiger Probanden. Stuttgart: ibidem.

Vollbrecht, Ralf (2001). Einführung in die Medienpädagogik. Weinheim: Beltz.

Wahl, Lisa (2012): Bearbeitungsprozesse einer textbasierten Lernaufgabe im Englischen. Ein Vergleich zwischen Kindern mit und ohne Migrationshintergrund. Unveröffentlichte Wiss. Hausarbeit i.R. der ersten Staatsarbeit. 
Wiedwald, Christian / Büsching, Nicole / Breiter, Andreas / Nöckel, Kerstin (2007). Pädagogische Medienentwicklungsplanung am Beispiel des Schulaufsichtsbezirks Frankfurt am Main: Zwischenbericht zur Mediennutzung in Schulen der Stadt Frankfurt am Main aus Sicht der Lehrkräfte. Bremen: Institut für Informationsmanagement. 


\title{
Belgian CLIL Teachers' Professional Identity
}

\author{
Eva De Mesmaeker / Katja Lochtman (Brussels)
}

\begin{abstract}
Content and language integrated learning (CLIL) is a generic term referring to a teaching approach whereby content is taught through a second or foreign language. For Belgian teachers entering the CLIL classroom, this dual-focused approach is a challenge, since they are either trained as content or language experts. This demands a professional (re)orientation which current teacher training programs in Belgium do not yet offer. How CLIL teachers interpret this multiple role and how they deal with it in practice, constitutes their professional identity. Insight in CLIL teachers' professional identity is expected to be useful in helping them to cope with the 'CLIL-challenge' by contributing to the development of teacher training programs for CLIL.

Bilinguales Lernen ist ein Unterrichtsansatz, der das Unterrichten eines Sachfachs in einer Fremdsprache anvisiert. Diese Herangehensweise, im Zuge derer sowohl Fachinhalte als auch Sprache zu unterrichten sind, scheint aber immer noch eine große Herausforderung für belgische Lehrerinnen und Lehrer zu sein, weil sie oft entweder als Sachfach- oder als Fremdsprachenlehrkräfte ausgebildet werden. Folglich wird von ihnen in einem CLIL-Programm eine gewisse professionelle Umorientierung erwartet. Dieser Beitrag untersucht die professionelle Identität bzw. die Auffassungen von CLILLehrkräften in Bezug auf diese duale Rolle und die entsprechende Unterrichtspraxis. Die Ergebnisse bieten neue Erkenntnisse für die Ausbildung von Lehrkräften in einem bilingualen Unterricht.
\end{abstract}

\section{$1 \quad$ Introduction}

Content and language integrated learning (CLIL) is a generic term referring to a teaching approach whereby content is taught through a second or foreign language. Compared to other forms of foreign language teaching (FLT), "in essence, its distinctiveness lies in an integrated approach, where both language and content are conceptualized on a continuum without an implied preference for either" (Coyle 2007: 545). This drive for integration is "a powerful pedagogic tool which aims to safeguard the subject being taught whilst promoting language as a medium for learning as well as an objective of the learning process itself" (Coyle 2002: 27). For Belgian teachers entering the CLIL classroom, however, the integration of content and language learning implies a significant change in their mindset, since they are either trained as content or language experts. They have to deal with a situation that moves away from the traditional view on education where each discipline tends to stand on its own. As a result, we see that content teachers, who are not familiar with second language acquisition theories, predominantly focus on subject matter, 
while language teachers display an overemphasis on linguistic form (Coyle 2007).

While "the study of teacher cognition has established itself on the research agenda in the field of language teaching and provided valuable insight into the mental lives of language teachers" (Borg 2003: 81), research into teachers' attitudes and beliefs in CLIL is still rather scarce but is getting increasingly more attention. The aim of the present exploratory study is to contribute to understanding how CLIL-teachers perceive their professional experience, how they interpret their (multiple) roles and how they think they deal with it in practice. To answer this question, we would like to describe CLIL teachers' perceptions of their professional identity, a concept that is closely related to quality and innovation in education (Lamote/Engels 2010). Research has shown that teachers' perceptions of their own professional identity strongly affect their efficacy and professional development as well as their ability and willingness to cope with educational change and to implement innovations in their own teaching practice (Beijaard et al. 2004; Lamote/Engels 2010). This study is the first to examine CLIL teacher beliefs and attitudes in the Belgian context.

When a school wants to introduce CLIL education, it has to take a number of aspects into consideration, such as when to start, how many hours, which languages, etc. (Van de Craen et al. 2008). These decisions depend on various local factors and on language policies. In officially trilingual Belgium where the majority languages are Dutch and French, it is therefore logical that schools mainly concentrate on Dutch and French. Contrary to such geopolitical considerations, a number of Belgian schools have also adopted English as the favourite second language.

\section{CLIL pedagogy}

In order to provide a basis for bringing together the different facets of CLIL, Coyle $(2002,2007)$ developed the 4C's conceptual framework, in which she expounds the interrelationship between content (subject knowledge), communication (language knowledge), culture and cognition. According to Coyle (2007), however, a cohesive view of CLIL pedagogies is still lacking. She states that two general observations can be made about European CLIL in the last two decades. On the one hand, CLIL pedagogies have been highly influenced by language acquisition theories that favor language teaching perspectives. On the other hand, transmission-oriented approaches to CLIL in certain contexts have encouraged teachers to focus primarily on content delivery. As Lyster (2007: 1) points out, traditional teaching methods indeed "tend to separate language development from general cognitive development", ignoring the relationship between form and meaning, and between language learning and content learning. However, as Freeman (2002: 6) puts it: "when applied to language as 
subject matter, PCK [pedagogical content knowledge] becomes a messy and unworkable concept".

With the European Framework for the professional development of CLIL teachers (Marsh et al. 2010), an attempt has been made to formulate the professional competences a CLIL teacher is expected to acquire. It aims to provide a set of principles and ideas for designing CLIL professional development curricula. Nevertheless, this document states that the integrative nature of CLIL is highlighted as a major challenge in the development and implementation of a teacher education curriculum. We might thus conclude that applying CLIL the way it is conceptualized by researchers suggests significant changes in the scope of teachers' responsibilities. Teachers undertaking CLIL would need to develop multiple types of expertise: among others, in the content subject, in the target language, with regard to best practices in teaching and learning and in the integration of the previous three. But, off-the-shelf CLIL materials still are in short supply (Mehisto et al. 2008). Teachers would therefore often spend considerable time developing their own course material and/or adapting existing learning resources. Furthermore, changing the medium of instruction would place increased organizational and cognitive demands on CLIL teachers, as much as on their students (Mehisto 2008). The integration of content and language teaching would not be "self-evident" and it seems to require considerable training for both teachers and pupils (Gajo 2007: 578).

In Belgium, teachers are trained to teach just one subject, i. e. a content subject or a language. In the absence of formal training on integrating content and language teaching, teachers' beliefs could become a crucial factor in guiding their pedagogical classroom practices (Tan 2011) and could be viewed as a lens through which teachers perceive and filter external information (Beijaard et al. 2004; Lamote/Engels 2010). Therefore, it is the aim of the present study to investigate CLIL teachers' perceptions of their professional identity.

\section{The professional identity of teachers}

Teachers' beliefs, and therefore their professional identities, are found to have an influence on their teaching practice (Borg 2003). Sammons et al. (2007) even found a relationship between aspects of teachers' professional identity and pupils' attainment in English and mathematics. In general, we can say that the concept of professional identity refers to the perception that teachers have of themselves as teachers. It appears from the existing research, however, that the concept of identity has been difficult to define in a clear-cut way (Borg 2003). In their review of research on teachers' professional identities, Beijaard et al. (2004) point out that the concept has been used with different meanings and definitions in more general literature as well as in the domain of teaching and teacher education. On the one hand, authors have drawn on the definition of 
identity as used in the social sciences and philosophy, relating it to concepts of self (Erikson 1968) and to what is termed as personal identity (Korthagen 2004) and self-understanding (Kelchtermans 2005). However, as Lamote/Engels (2010: 4) put it, "in an occupation where who one is as a person is so much interwoven with how one acts as a professional, both sides cannot be separated". "It seems unlikely that the core of the personal will not impact on the core of the professional" (Loughran 2006: 112). Furthermore, Beijaard et al. (2004) argue that a teacher's professional identity is not unitary but consists of sub-identities formed through a teacher's different contexts and relationships. "Some of these sub-identities may be broadly linked and can be seen as the core of teachers' professional identity, while others may be more peripheral" (Beijaard et al. 2004: 122). Essential for teachers is that these sub-identities do not conflict, but that they more or less harmonize.

Another feature of professional identity is the idea that the construct is dynamic and can be seen as an ongoing process of interpretation and reinterpretation. This corresponds to the notion that teacher development never stops and can best be seen as a process of lifelong learning (e. g. Day 2002). In this study teachers' professional identity will be described in terms of the CLIL teacher's perceptions of task orientation, professional orientation and selfefficacy, three indicators for which we find support in the literature on the professional identity of teachers (Lamote/Engels 2010).

\subsection{Professional orientation}

By professional orientation we mean perceptions of teachers about their professional role(s) in a broader sense, not limited to their classroom practice but with regard to organizational aspects of the school context. In that sense, professional orientation is not a stable indicator of professional identity, since views of the teaching profession and the role of teachers have changed over time. One example is that a teacher's role has changed from transferring knowledge to facilitating learning processes. Moreover, during the past decades, the roles and responsibilities of teachers have broadened extensively (OECD 2005). Present-day teachers are expected to be concerned with collaborative work in the school team, to contribute to school policy making, to expand their knowledge by looking for other theories and methods, to collaborate with parents and external partners, and so much more. This view of the teacher who reflects and acts on a broader level, showing him- or herself to be creative, an innovator and team player contributing to school development, corresponds to Hoyle's (1980) conception of the 'extended professional', whereby extended professionals are expected to be more flexible with regard to educational innovations. As we mentioned in the introduction, CLIL teachers are facing significant challenges in the scope of their responsibilities. In this study we 
therefore assume professional orientation to be an important descriptor for CLIL teachers' professional identity.

\subsection{Task orientation}

In line with the conceptualization of teachers' professional identities by Lamote/Engels (2010), we consider task orientation as referring to personal theories teachers have with regard to their core task. Task orientation refers to teachers' answers to the questions: 'What do I want to achieve with my pupils and how do I want to do this?' Like professional orientation, task orientation is influenced by the prevailing knowledge and beliefs about the goals and teaching methods at a certain time. The current reforms regarding teaching methods are strongly dominated by constructivist as opposed to behaviorist theories, resulting in two different views of knowledge, learning and teaching methods and a pupil-oriented approach vs. a content-oriented approach. From the perspective of a content-oriented ideology, the teacher focuses on discipline in the classroom, on preparing pupils to take their responsibilities in society and on developing competences and skills in core subjects. The pupil-oriented view mainly emphasizes pupil involvement, personal development of pupils, creativity and active knowledge acquisition. In our attempt to capture current beliefs about good education, we build on Denessen's (1999) construct of task orientation, which distinguishes between three different aspects of teaching: (1) the educational goals, (2) the instructional emphasis and (3) the pedagogical relation between teachers and students, for each of which he describes a more studentoriented approach vs. a more content-oriented approach.

With regard to the specific task orientation of CLIL teachers, characterized by a dual-focused approach on content and language learning, the following documents may serve as a reference: the European Framework for CLIL Teacher Education (Marsh et al. 2010), An observation tool for effective L2 pedagogy in CLIL (de Graaff et al. 2007) and the Standard for Bilingual Education (European Platform 2012). The proposed items tend to capture a more vs. a less integrated approach of content and language learning.

\subsection{Self-Efficacy}

Tschannen-Moran/Woolfolk Hoy (2001: 783) define a teacher's self-efficacy as "a judgment of his or her capabilities to bring about desired outcomes of student engagement and learning, even among those students who may be difficult or unmotivated". This definition builds on Bandura (1977) who introduced the conceptual framework and asserted that efficacy beliefs were more powerful than a person's actual abilities for the task at hand. Teachers' sense of efficacy 
has proven to be a powerful construct, related to many meaningful educational outcomes such as teachers' motivation (persistence, commitment, enthusiasm) and instructional behavior in the classroom. Teachers with high levels of selfefficacy would work longer with students that struggle, would recognize student errors and would attempt new teaching methods that support students (Guskey 1988) and they would be more willing to adopt instructional innovation (Guskey/Passaro 1994). In addition, a high level of self-efficacy is said to contribute to important student outcomes such as achievement, motivation and their own sense of efficacy (Tschannen-Moran et al. 1998). Self-efficacy beliefs turn out to be affected by four major sources - previous successful experiences, indirect experiences (e. g. modeling by someone else), persuasion by others and emotional feedback (Tschannen-Moran/Woolfolk Hoy 2001). Research has also shown that teachers' self-efficacy beliefs are situation-specific in that a teacher can hold divergent efficacy beliefs for different teaching situations. Efficacy beliefs may vary within teachers depending upon the subject area, characteristics of students, the number of course preparations they face, and whether they are teaching outside the field of expertise (Ross et al. 1999; Tournaki/Podell 2005; Tschannen-Moran/Johnson 2011).

\section{The study}

\subsection{Method}

\section{Participants}

The participants are Belgian secondary school CLIL-teachers $(\mathrm{N}=80)$ from 45 schools in Flanders (5), Brussels (9), and Wallonia (31) who completed a questionnaire in the period between May-June 2012. The distribution of male and female respondents was $38,8 \%$ and $61,3 \%$, respectively. The average age was $39(\mathrm{SD}=9.3)$, the average amount of experience in secondary education was 9 years $(\mathrm{SD}=7.9)$ and the average amount of experience in CLIL education was 4.5 years $(\mathrm{SD}=3.2)$. For $60 \%$ of the respondents the CLIL target language is their mother tongue.

\section{Research tool}

The participants completed an online survey which can be split up in two larger sections. In the first section respondents were asked for information concerning several background variables on the one hand: age, gender, mother tongue, language knowledge, academic background, teaching experience in secondary education and more specifically in CLIL education and school contextual variables on the other hand: CLIL target language, CLIL subjects, teaching materials being used, professionalization and training, professional support and collegial interaction. The second section consisted of several scales, for which 
respondents had to answer every statement on a five-point Likert scale. The scales measuring the dimensions of professional identity - task orientation, professional orientation and self-efficacy - are described below.

\section{Professional orientation}

Professional orientation of the CLIL-teachers was measured using the 13-item scale developed by Jongmans/Beijaard (1997). We decided to convert this scale - originally scoring on a four-point scale - to a five-point Likert scale, in conformity with the other scales being used. The final score reflects the levels of professional orientation. Based on Hoyle's (1980) conception, a high score (strongly agree) stands for an 'extended professional'. A sample item is: 'It is important for teachers to attend in-service training courses'.

\section{Task orientation}

For the assessment of the general task orientation of CLIL-teachers we used the Views on education scale developed by Denessen (1999). This scale has 25 items, which are subdivided into three areas: educational goals, instructional emphasis and pedagogical relationship. Sample items are: 'It is the school's responsibility to teach students a critical attitude toward society' (educational goals - personal and social development); 'If children want to make something of their lives, they need to learn a lot at school' (educational goals - career development); 'Students can also learn a great deal from each other' (instructional emphasis - process); 'I believe competition between students is important to foster learning' (instructional emphasis - product); 'I think it is important that students are above all diligent and make an effort' (pedagogical relationship - discipline); 'I think student participation in school matters is important' (pedagogical relationship - involvement).

To measure the task orientation of CLIL teachers we developed items for several areas of the CLIL-approach: learning goals, didactic approach, feedback and evaluation, language awareness strategies. All task orientation items had to be read as statements starting by 'I believe it is my responsibility as a CLIL teacher...'. Sample items in the different areas are: ' ... to set clear content goals as well as language goals for each lesson' (CLIL learning goals); ' ... to opt for learning activities that offer opportunities for language development' (in terms of the four language skills: speaking, writing, reading, listening (didactic approach)); '... to give corrective feedback on pupils' oral language production' (feedback); ' . . to make pupils aware of the differences and similarities between languages' (language awareness strategies). Depending on a high score (strongly agree) or a low score (strongly disagree), respondents could be regarded as more 
or less oriented towards an integrated approach of content and language learning.

\section{Self-efficacy}

CLIL teachers' efficacy beliefs for more general aspects of teaching were measured using the Teachers' Sense of Efficacy Scale (short version) (Tschannen-Moran/Woolfolk Hoy 2001). This measure assesses three dimensions of the underlying construct: sense of efficacy for instructional strategies, sense of efficacy for stimulating pupils' involvement and sense of efficacy for classroom management. Each statement had to be read from this viewpoint, 'As a CLILteacher, to what extent do you feel able ...' and had to be scored on a 5-point response scale $(1=$ not at all, $2=$ very little, $3=$ somewhat, $4=$ quite a bit, $5=\mathrm{a}$ great deal. Sample item: '... to motivate students who show little interest in school work?'

For the assessment of teachers' sense of efficacy about CLIL instructional strategies, a 17-item survey was composed based on the same documents used for the development of the CLIL task orientation survey. The aim was to cover several aspects with regard to effective CLIL pedagogy. The statements had to be read from the viewpoint: 'As a CLIL-teacher, to what extent do you feel able ...'. Sample items are: '... to reach the core goals of the course by using authentic materials in the target language'; '... to use didactic strategies that push the language output of pupils in the target language'; '.. to use corrective feedback to make pupils aware of their linguistic errors'; '... to use a level of language proficiency that complies with the students' level of comprehension'; '... to set assignments and tests to evaluate the pupils' progress with regard to language as well as the subject area'. Additionally, CLIL- teachers were asked about their perceived pedagogical role within the CLIL classroom on a 7-point Likert scale ranging from $1=$ 'I see myself exclusively as a content teacher' to 7 $=$ 'I see myself exclusively as a language teacher'.

Another important contextual variable that was added to the questionnaire is that of collegiality among teachers. Teacher collegiality, which can be described as the quality and impact of professional relationships whereby teachers openly and continually investigate and critique school/classroom practice with a view to improvement (Lieberman/Miller 1999), is accepted as essential to professional practice. Recent research has suggested that developing the ability of school personnel to function as professional collegial communities may result in substantive school improvement and success (DuFour 2004; Goddard et al. 2007; Little et al. 2003). Strengthening interpersonal relations among teaching personnel is thought to influence a school's professional culture and lead to teachers increasing their involvement and ownership (Andrews/Lewis 2002). 
Furthermore, collegiality is said to play a significant role in improving teaching and instructional practices and fostering innovation (Hopkins et al. 1998).

From the assumption that improvement in teaching is a collective endeavor rather than an isolated event, Little (1990) created a model explaining a school's journey from independence to interdependence. Her model included four forms of collegiality: storytelling and scanning for ideas, aid and assistance, sharing and joint work, which she put on a continuum ranging from activities that are compatible with teacher independence and autonomy to activities that require interdependent action and are believed to have great potential to build a schoolwide culture of collegiality. Based on Little's model, we developed a scale with 14 items, measuring the level of collegial interaction. Sample items are: 'I exchange ideas on education with colleagues', 'Sometimes I ask a colleague for help with regard to my teaching practice', 'I swap teaching materials with colleagues', 'Within our CLIL team we share responsibility for the design of our CLIL courses'.

\subsection{Results and discussion}

We start the description of the results with a report of the factor structure of each instrument. For all of the scales a principal component analysis (PCA) with orthogonal rotation (varimax) was conducted. The Kaiser-Meyer-Olkin measure verified the sampling adequacy for the analysis. The internal consistency of the scales and subscales was verified by a test of alpha reliability and by checking the theoretical framework.

\section{Professional orientation}

A PCA of the items assessing the professional orientation of CLIL teachers provided us with three components based on the Kaiser criterion, which we labeled 'professionalization' ( 5 items, $\alpha=0.867$ ), 'team policy' ( 3 items, $\alpha=$ 0.629 ) and 'cross-curricular cooperation' ( 5 items, $\alpha=0.724$ ).

\section{Task orientation}

First, we performed a PCA for the three general dimensions of task orientation. For the items measuring objectives of education, the PCA provided two components based on the Kaiser criterion. By analogy with the theoretical concept taken from Denessen (1999), we labeled component 1 as 'personal and social development' ( 6 items, $\alpha=0.832$ ) and component 2 as 'career development' (4 items, $\alpha=0.844)$. A PCA of the items assessing the pedagogical relationship revealed a three component structure, which we reduced to two components as only two items were related to component 3. In accordance with Denessen's re- 
search (1999), we named the first component 'discipline' (5 items, $\alpha=0.752$ ) and the second component 'involvement' ( 3 items, $\alpha=0.731$ ). A PCA of the items measuring the instructional emphasis showed a structure with two components, the first of which we referred to as 'process' ( 4 items, $\alpha=0.758$ ) and the second 'product' ( 3 items, $\alpha=0.418$ ). Since the internal consistency of the 'product' scale scores under the acceptable standard of $\alpha \geq .60$, we excluded it from further analysis.

Another PCA was conducted for the 16 items assessing CLIL-teachers' task orientation, which provided us with four components based on the Kaiser criterion. Since the fourth component consisted of only two items, we decided to reduce the number of factors to three. After an interim analysis of internal consistency, the three-factor structure seemed to be the best solution. We labeled them: 'language awareness strategies' ( 6 items, $\alpha=0.843$ ), 'goals and instruction' ( 6 items, $\alpha=0.795$ ), and 'stimulating input and output' ( 4 items, $\alpha=$ $0.711)$.

\section{Self-efficacy}

First, a PCA for the 12 items assessing teachers' sense of efficacy about the general aspects of teaching revealed a three-factor structure. By analogy with Tschannen-Moran/Woolfolk Hoy (2001), we named the first component 'sense of efficacy for instructional strategies' ( 3 items; $\alpha=0.691$ ), the second 'sense of efficacy for stimulating pupils' involvement' (4 items, $\alpha=0.762)$ and the third component 'sense of efficacy for classroom management' (5 items, $\alpha=0.837$ ).

Based on a second PCA, teachers' sense of efficacy about CLIL instructional strategies, measured on a five-point Likert scale, was split into four components based on the Kaiser criterion. We labeled them: 'form and meaning-focused processing' ( 6 items, $\alpha=0.842$ ), 'scaffolding and feedback' ( 5 items, $\alpha=$ $0.810)$, 'selecting and adapting material' ( 3 items, $\alpha=0.754$ ) and 'stimulating input and output' ( 3 items, $\alpha=0.734$ ).

\section{Collegiality}

A PCA was conducted for the 14 items informing about collegiality in the school team, which provided a three-factor structure. In line with Little's model on collegiality (1990), we labeled the first component 'Sharing, aid and assistance' ( 5 items, $\alpha=0.735$ ), the second 'Joint responsibility for work' (5 items, $\alpha=0.830$ ) and the third 'Storytelling and scanning for ideas' ( 4 items, $\alpha=$ $0.835)$. 
Table 1 (cf. next page) summarizes the internal consistency, explained variance, mean and standard deviation for different subscales measuring professional identity of CLIL-teacher. The scores of CLIL-teachers on the professional orientation scales turned out to be moderate to low (Figure 1: M (= mean score) items). Acording to Jongmans/Beijaard (1997, based on Hoyle 1980), a low score on these scales represents a 'restricted professional' profile, teachers who are primarily focused on the classroom and mainly concerned with their own teaching activities, didactic behaviors and subject matter. If we consider the different subscales of professional orientation, we notice that the rather low score particularly pertains to what we labeled the 'cross-curricular cooperation' scale, with items asking for teachers' beliefs about the importance of cooperation between colleagues and cross-curricular tasks. This low score is in line with the low score of CLIL teachers on the scale assessing collegiality in their school context. Keeping in mind Little's model (1999), we can say that CLIL teachers, in average, seem to be at one end of the curriculum, i. e. the 'independence' side (as opposed to the 'interdependence' side), implying that they are working autonomously in a school culture with little collegial interaction or feelings of joint responsibility about the development and practice of the CLIL curriculum. Rather moderate are the scores on the professionalization subscale, which indicates that the teachers are open to innovation, prepared to read professional journals and follow extra training. 
Table 1: Internal consistency, explained variance, mean and standard deviation for different subscales measuring professional identity of CLIL-teachers

\begin{tabular}{|c|c|c|c|c|c|c|}
\hline Area & Scale & $\begin{array}{c}N \\
\text { items }\end{array}$ & $\begin{array}{c}C \\
\text { alpha }\end{array}$ & $\begin{array}{c}\% \\
\text { explained } \\
\text { variance }\end{array}$ & $\begin{array}{c}M \\
\text { items }\end{array}$ & $\begin{array}{c}S D \\
\text { items }\end{array}$ \\
\hline \multicolumn{7}{|l|}{ Task orientation (general) } \\
\hline \multirow[t]{2}{*}{ Educational goals } & $\begin{array}{l}\text { Personal and social } \\
\text { development }\end{array}$ & 6 & 0.832 & 37.1 & 3,34 & 0,56 \\
\hline & Career development & 4 & 0.844 & 23.6 & 2,69 & 0,76 \\
\hline \multirow[t]{2}{*}{ Instructional emphasis } & Process & 4 & 0.758 & 35.7 & 3,50 & 0,48 \\
\hline & Product & 3 & 0.418 & 20.3 & 3,06 & 0,66 \\
\hline \multirow[t]{2}{*}{ Pedagogical relationship } & Discipline & 5 & 0.752 & 33.4 & 3,31 & 0,49 \\
\hline & Involvement & 3 & 0.731 & 26.5 & 2,92 & 0,58 \\
\hline \multirow[t]{3}{*}{ Task orientation CLIL } & Language awareness strategies & 6 & 0.843 & 37.7 & 3,11 & 0,64 \\
\hline & Goals and instruction & 6 & 0.795 & 10.2 & 3,13 & 0,62 \\
\hline & Stimulating input and output & 4 & 0.711 & 9.1 & 3,31 & 0,59 \\
\hline \multirow[t]{3}{*}{ Professional orientation } & Professionalization & 5 & 0.867 & 43.3 & 3,07 & 0,65 \\
\hline & Cross-curricular cooperation & 5 & 0.724 & 10.2 & 2,56 & 0,91 \\
\hline & Team policy & 3 & 0.629 & 8.0 & 2,95 & 0,68 \\
\hline \multirow[t]{3}{*}{ Self-efficacy (general) } & Class management & 5 & 0.837 & 42.9 & 2,97 & 0,57 \\
\hline & Student engagement & 4 & 0.762 & 12.5 & 2,53 & 0,55 \\
\hline & Instructional strategies & 3 & 0.691 & 8.5 & 2,86 & 0,57 \\
\hline \multirow[t]{4}{*}{ Self-efficacy (CLIL) } & $\begin{array}{l}\text { Form and meaning focussed } \\
\text { processing }\end{array}$ & 6 & 0.842 & 42.4 & 3,55 & 0,69 \\
\hline & Scaffolding and feedback & 5 & 0.810 & 9.5 & 3,76 & 0,60 \\
\hline & Selecting and adapting material & 3 & 0.754 & 6.8 & 3,67 & 0,68 \\
\hline & Stimulating input and output & 3 & 0.734 & 6.3 & 3,73 & 0,67 \\
\hline \multirow[t]{3}{*}{ Collegial interaction } & Sharing, aid and assistance & 5 & 0.735 & 36.2 & 2,90 & 0,65 \\
\hline & Joint responsibility & 5 & 0.830 & 17.3 & 2,17 & 0,98 \\
\hline & $\begin{array}{l}\text { Storytelling and scanning for } \\
\text { ideas }\end{array}$ & 4 & 0.835 & 11.6 & 2,72 & 0,85 \\
\hline Professional support & & 4 & 0.747 & & 2,34 & 0,88 \\
\hline
\end{tabular}

CLIL-teachers' scores on the general task orientation scales are rather moderate as well, although more tending towards a pupil-oriented approach to teaching (Table 1: $\mathrm{M}$ items). They seem to emphasize personal and social development of their students and are in favor of a process-oriented approach in the classroom. Concerning the student-teacher relationship, on the other hand, CLIL-teachers seem not to agree with pupils' taking too much part in decision-making at school, but rather want to maintain discipline in the classroom. With regard to the specific CLIL task orientation subscales, the same moderate results hold true. Teachers neither agree nor disagree markedly with the statements that, for example, raising language awareness by using different strategies or setting clear content and language goals, are part of their responsibilities as a CLIL teacher. 
With reference to their self-efficacy, CLIL teachers attribute themselves rather low scores on the scales measuring their sense of efficacy beliefs about the general aspects of teaching (Figure 1: $\mathrm{M}$ items). Especially with regard to the subscale 'student engagement' $(\mathrm{M}=2.53)$, indicating that one is able to keep students motivated for school work and to help them appreciate the value of learning, CLIL teachers believe to have rather little influence. Remarkably, the opposite is true for teachers' sense of efficacy about the CLIL approach, for which they attribute themselves moderately high scores on the different subscales, ranging from 3.55 to 3.76 .

When asking respondents about the CLIL subjects they taught at their school, we noticed that part of the teachers indicated that they teach both content classes and language classes within the CLIL program. By language classes we mean English, French or Dutch as a foreign language, but also conversation classes or writing and reading courses in the target language. Apart from teaching biology and chemistry, for example, some teachers indicated that they provided language-based courses. In the analysis, teachers were therefore divided into two groups on the basis of whether they only taught a content class $(\mathrm{N}=41)$, or whether they also taught a language class in addition to teaching a content class $(\mathrm{N}=21)$.

The results of the scale where respondents had to indicate their perceived pedagogical role within the CLIL classroom on a 7-point Likert scale ranging from 1 = 'I see myself exclusively as a content teacher' to $7=$ 'I see myself exclusively as a language teacher', show that teachers' beliefs about their respective roles as mainly content teachers or mainly language teachers depend on the types of courses they teach (figure 1). Those respondents who teach only content classes see it as their principal pedagogical role to teach content, whereas those who also teach a language course have a much more balanced, 'integrated' approach to teaching content and language. As research has shown, these perceptions may have implications for the actual instructional practices of these teachers. Especially in the case of the content teachers, whose pedagogical beliefs show little regard for the important role of language in the teaching and learning process, their classroom practices may limit students' (language) learning opportunities. In fact, both content and language teachers can offer expertise to CLIL, but as for example Mehisto (2008: 104) concludes from extensive lesson observations and debriefing sessions with teachers in Estonian CLIL programs, "many teachers appear to have a mindset that does not readily leave room for taking on an expanded role as both a teacher of content and language". It is possible that many content teachers do not recognize that content acquisition is inextricably tied to language learning and that every content lesson needs to be a language lesson. As Lyster (2007: 5) remarks on the strategic manipulation of language in immersion and content-based classrooms "[s]uch an approach requires a great deal of systematic planning and does not 
necessarily come naturally to content-based teachers". Indeed, different teachers may have very different representations of the role and function of language in learning and teaching, with "far reaching consequences for the language ecology of their classroom and therefore also for the language opportunities which arise in them" (Dalton-Puffer 2008: 12).

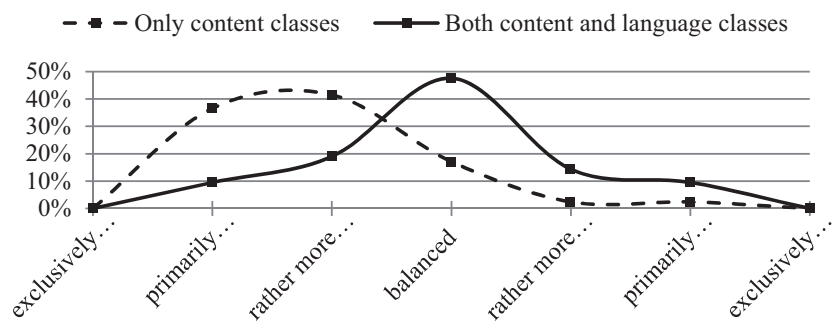

Figure 1: Percentages of teachers teaching only content classes and those teaching both content and language classes who see themselves as either more a content teacher or a language teacher.

\section{Conclusion}

The main focus of the present study was to describe Belgian CLIL teachers' perceptions of their professional identity. More specifically, our aim was to explore CLIL teachers' beliefs about the professional characteristics of the content and language integrated approach. In this article, we report on quantitative data collected through an online survey. This survey was distributed among secondary CLIL teachers in both the Dutch and the French-speaking community of Belgium.

Three professional characteristics were considered as representations of teachers' professional identity: their professional orientation, task orientation and self-efficacy. For the measurements of these characteristics, we made use of multiple item scales that were found valid and reliable in previous research. As both the construct self-efficacy and task orientation demanded specific items with regard to the integrated approach of CLIL, we developed separate scales for the assessment of teachers' sense of efficacy about CLIL instructional strategies and task orientation for goals, methods and strategies that are considered necessary for the multiple focus in CLIL.

Results show that CLIL teachers in the Belgian context describe themselves as having a moderate view on general task orientation, tending towards a pupiland process-oriented approach of teaching. The same moderate scores appear for the scales measuring CLIL specific instructional orientations, where respondents seem to have no marked opinion about the use of language awareness strategies, 
goals and instructional methods for the integrated approach or the stimulation of input and output. With regard to their sense of efficacy, CLIL teachers attribute themselves moderately low scores for self-efficacy with regard to the general aspects of teaching, as opposed to moderately high scores for teachers' efficacy with regard to specific aspects of CLIL teaching. Since self-efficacy is regarded as a powerful construct related to teachers' motivation and behavior in the classroom as well as contributing to important student outcomes (Tschannen-Moran et al. 1998), further research is needed to shed light on how these efficacy beliefs are established and what factors may lead to their improvement. As a positive linear correlation was found with the level of collegiality in the school context, opportunities for raising self-efficacy beliefs undoubtedly lie in helping school teams to function as professional collegial communities, working together in an atmosphere of joint responsibility (Andrews/Lewis 2002).

Another interesting finding concerns the CLIL teachers' perceived pedagogical role within the CLIL classroom. Teachers who provide both content and language classes in the CLIL curriculum seem to have a more balanced, 'integrated' view on teaching content and language as opposed to teachers who exclusively teach the content subject they were trained for. A greater focus on the integration of content and language in educational literature and teacher training could help to change the fixed mindset, separating language learning form content learning, for future generations of teachers. This is also a plea for the integration of general pedagogy and subject specific pedagogy, which are often treated as separate areas of expertise in Belgian teacher training programs.

Finally, we deal with the limitations linked to the method that was used in this study. First, when using self-reporting instruments, there is always the risk that respondents provide 'socially' desirable answers. Second, the length of the questionnaire (estimated time: $40 \mathrm{~min}$.) might have been responsible for respondents not to be as reflective and accurate until the last question. Furthermore, the closed structure of a questionnaire with set answering categories leaves little room for specifications or nuances. Although we added space for remarks and several open questions, most data was gathered through multiple choice questions and multiple item Likert scales, so that respondents only had to tick boxes to state their beliefs. In their review study, Beijaard et al. (2004) assert that "a structured though open method of data collection" to capture teachers' professional identity seems to be most desirable. They further emphasize the relevance of stories and narratives in research on teachers' professional identity. Therefore the present quantitative study should be extended with qualitative research, comprising interviews with teachers, classroom observations and the use of stimulated recall (see also Borg 2003). Also relevant for further research is the exploration of teachers' professional identity from the perspective of other stakeholders. Since professional identity is socially defined, shaped through interaction with others and with the 
environment, it would be interesting to explore perspectives of all those involved (school management, educators, trainers, parents, students, policy makers, etc.).

\section{References}

Andrews, Dorothy / Lewis, Marian (2002). The experience of a professional community: Teachers developing a new image of themselves and their workplace. Educational Research 44/3: 237-254.

Bandura, Albert (1977). Self-efficacy: towards a unifying theory of behavioral change. Psychological Review 84/2: 191-215.

Beijaard, Douwe / Meijer, Pauline C. / Verloop, Nico (2004). Reconsidering research on teachers' professional identity. Teaching and Teacher Education 20/2: 107-128.

Borg, Simon (2003). Teacher cognition in language teaching: A review of research on what language teachers think, know, believe, and do. Language Teaching 36: 81-109.

Coyle, Do (2002). Relevance of CLIL to the European Commission's language learning objectives. In: CLIL/EMILE, the European dimension: Action, Trends and Foresight Potential. Public Services Contract DG EAC. Strasbourg: European Commission.

Coyle, Do (2007). Towards a connected research agenda for CLIL pedagogies. The International Journal of Bilingual Education and Bilingualism 10/5: 543-562.

Dalton-Puffer, Christiane (2008). Outcomes and processes in content and language integrated learning (CLIL): current research from Europe. In: Delanoy, Werner / Volkmann, Laurenz (eds.): Future Perspectives in English Language Teaching. Heidelberg: Carl Winter. 1-19.

Day, Christopher (2002). School reform and transitions in teacher professionalism and identity. International Journal of Educational Research 37: 677-92.

De Graaff, Rick / Koopman, Gerrit Jan / Anikina, Yulia / Westhoff, Gerard (2007). An observation tool for effective L2 pedagogy in CLIL. The International Journal of Bilingual Education and Bilingualism 10/5: 603-624.

Denessen, Eddie (1999). Opvattingen over onderwijs. Leerstof - en leelinggerichtheid in Nederland. Leuven: Garant Uitgevers.

DuFour, Richard (2004). What is a "professional learning community?". Educational Leadership 61/8: 6-11.

Erikson, Erik (1968). Identity, Youth and Crisis. New York: W.W. Norton \& Company.

European Platform (2012). Standard for Bilingual Education. Haarlem: European Platform.

Eurydice (2006). Content and Language Integrated Learning (CLIL) at School in Europe. Brussels: Eurydice.

Freeman, Donald (2002). The hidden side of the work: Teacher knowledge and learning to teach. Language Teaching 35: 1-13.

Gajo, Laurent (2007). Linguistic knowledge and subject knowledge: How does bilingualism contribute to subject development? International Journal of Bilingual Education and Bilingualism 10/5: 563-81. 
Goddard, Yvonne L. / Goddard, Roger D. / Tschannen-Moran, Megan (2007). A theoretical and empirical investigation of teacher collaboration for school improvement and student achievement in public elementary schools. Teachers College Record 109/4: 877-896.

Guskey, Thomas R. (1988). Teacher efficacy, self-concept, and attitudes toward the implementation of instructional innovation. Teaching and Teacher Education 4: 63-69.

Guskey, Thomas R. / Passaro, Perry D. (1994). Teacher efficacy: A study of construct dimensions. American Educational Research Journal 31/3: 627-43.

Hopkins, David / Beresford, John / West, Mel (1998). Creating the conditions for classroom and teacher development. Teachers and Teaching: Theory and Practice 4/1: 115-141.

Hoyle, Eric (1980). Professionalization and deprofessionalization in education. In: Hoyle, Eric / Megarry, Jacquetta (eds.): World Yearbook of Education 1980, Professional Development of Teachers. London: Kogan Page. 42-57.

Jongmans, Kees / Beijaard, Douwe (1997). De professionele oriëntatie van leraren en hun betrokkenheid bij het schoolbeleid. Pedagogische Studiën 74/2: 97-107.

Kelchtermans, Geert (2005). Teachers' emotions in educational reforms: Self-understanding, vulnerable commitment and micropolitical literacy. Teaching and Teacher Education 21: 995-1006.

Korthagen, Fred (2004). In search of the essence of a good teacher: Towards a more holistic approach in teacher education. Teaching and Teacher Education 20: 77-97.

Lamote, Carl / Engels, Nadine (2010). The development of student teachers' professional identity. European Journal of Teacher Education 33/1: 3-18.

Lieberman, Anne / Miller, Lynne (1999). Teachers: Transforming their World and their Work. New York: Teachers College Press.

Little, Judith W. (1990). The persistence of privacy: autonomy and initiative in teachers' professional relations. Teachers College Record 91/4: 509-536.

Little, Judith W. / Gearhart, Maryl / Curry, Marnie / Kafta, Judith (2003). Looking at student work for teacher learning, teacher community and school reform. Phi Delta Kappan 85/3: 184-192.

Loughran, John (2006). Developing a Pedagogy of Teacher Education. Understanding Teaching and Learning about Teaching. New York: Routledge.

Lyster, Roy (2007). Learning and Teaching Languages Through Content, a Counterbalanced Approach. Amsterdam: John Benjamins.

Marsh, David / Mehisto, Peeter / Wolff, Dieter / Frigols Martin, Maria J. (2010). European Framework for CLIL Teacher Education: A Framework for the Professional Development of CLIL teachers. Graz: European Centre for Modern Languages.

Mehisto, Peeter (2008). CLIL Counterweights: recognizing and decreasing disjuncture in CLIL. International Journal of Bilingual Education and Bilingualism 1/1: 93-119.

Mehisto, Peeter / Marsh, David / Frigols Martin, Maria J. (2008). Uncovering CLIL, Content and Language Integrated Learning in Bilingual and Multilingual Education. Oxford: Macmillan Education.

OECD (2005). Teachers Matter. Attracting, Developing and Retraining Effective Teachers. Paris: OECD Publishing. 
Ross, John A. / Cousins, Bradley / Gadalla, Tahany / Hannay, Lynne (1999). Administrative assignment of teachers in restructuring secondary schools: the effect of out-of-field course responsibility on teacher efficacy. Educational Administration Quarterly 35: 782-804.

Sammons, Pam / Day, Christopher / Kingston, Alison / Gu, Qing / Stobart, Gordon / Smees, Rebecca (2007). Exploring variations in teachers' work, lives and their effects on pupils: Key findings and implications from a longitudinal mixed-method study. British Educational Research Journal 33/5: 681-702.

Tan, May (2011). Mathematics and science teachers' beliefs and practices regarding the teaching of language in content learning. Language Teaching Research 15/3: 325-42.

Tournaki, Nelly / Podell, David, M. (2005). The impact of student characteristics and teacher efficacy on teachers' predictions of student success. Teaching and Teacher Education 21: 299-314.

Tschannen-Moran, Megan / Johnson, Denise (2011). Exploring literacy teachers' self-efficacy beliefs: potential sources at play. Teaching and Teacher Education 27:751-761.

Tschannen-Moran, Megan / Woolfolk Hoy, Anita (2001). Teacher Efficacy: capturing an elusive construct. Teaching and Teacher Education 17: 783 - 805.

Tschannen-Moran, Megan / Woolfolk Hoy, Anita / Hoy, Wayne K. (1998). Teacher efficacy: its meaning and measure. Review of Education Research 68: 202-248.

Van de Craen, Piet / Ceuleers, Evy / Lochtman, Katja / Allain, Laure (2008). An interdisciplinary approach to CLIL learning in primary schools in Brussels. In: Dalton-Puffer, Christiane / Smit, Ute (eds.): Empirical Perspectives on CLIL Classroom Discourse. Frankfurt/M.: Lang. 253-274. 
III.

Teacher Education and the Development of Multiliteracies 



\title{
Formations hybrides en milieu pluriculturel: comment concilier théories, pratiques et contraintes
}

\author{
Jean-Paul Narcy-Combes / Marie-Françoise Narcy-Combes \\ (Paris, Nantes)
}

\begin{abstract}
Après un tour d'horizon sur les théories sur lesquelles il s'appuie, cet article décrit quatre dispositifs d'apprentissage hybrides ou à distance où le savoir se construit collaborativement dans un contexte plurilingue et pluriculturel. Les résultats confirment les théories de références et soulignent la complexité de l'entreprise.

Following a brief state of the art, this article describes four distance or blended learning environments based on collaborative work in a mutilingual and multicultural context. The results are in keeping with what the literature describes and they highlight the complexity of such attempts.
\end{abstract}

\section{Problématique de l'article ${ }^{1}$}

L'objectif de cette réflexion exploratoire est de faire un état des lieux non exhaustif des conceptions actuelles sur l'apprentissage des langues et des contenus disciplinaires dans un contexte de plurilinguisme et d'interactions internationales, pour décrire quelques applications qu'elles ont inspirées dans nos universités. La problématique théorique sera présentée initialement, suivie par l'étude comparative de ces applications pour dégager les voies les plus prometteuses dans notre contexte, les problèmes que ces voies soulèvent néanmoins et les recherches qu'elles imposent. Chacun des projets a été accompagné d'un dispositif de recherche-action. Des données empiriques ont été recueillies et analysées, ou bien sont en cours d'analyse, cependant le présent article ne se donne pas pour objet de communiquer sur ce point, faute de place pour le faire.

\subsection{Relations transductives}

Trois relations transductives ${ }^{2}$ seront utiles pour comprendre la problématique de cet article. La première est celle que Simondon (1989) voit entre homme, outils

\footnotetext{
${ }^{1}$ Cette problématique doit beaucoup au travail de Lelouda Souliou, doctorante à Paris 3, et les auteurs lui sont reconnaissants de son travail et l'encouragent à vite publier.

${ }^{2}$ La transductivité dans une relation implique qu'aucun des éléments ne saurait exister sans les autres.
} 
ou technologie, et société. Simondon, comme d'autres (voir Narcy-Combes 2005) considère que le langage est une technologie. A la suite de cette relation, on pourrait en postuler une seconde entre langage, culture et savoir(s) car aucun de ces éléments ne saurait se concevoir sans les autres. En effet, la production langagière ne se fait pas pour elle-même: l'objectif des interactants est de négocier du sens sur un sujet donné, dans un domaine précis. Il restera à comprendre, dans cette optique, l'incidence de la connaissance du contenu disciplinaire traité par l'apprenant quand il travaille en L2, afin de déterminer des pratiques d'enseignement-apprentissage. Enfin, une troisième relation transductive existe entre parole, langue et langage. Le langage est la faculté que les humains ont développée pour communiquer, mais également organiser leur monde. La réalisation physique en est la parole ou le discours. Les groupes humains ont donné des réalisations physiques différentes au langage, les langues. En conclusion, il ne nous parait pas possible de dissocier l'apprentissage langagier de celui des disciplines ou des contenus et il ne nous parait pas plus possible de le faire en dehors de tout contexte culturel. Il nous reste à comprendre ce que signifie faculté de langage et production langagière dans un contexte plurilingue.

\subsection{Positionnement sur le plurilinguisme}

La compétence plurilingue et pluriculturelle a des bases empiriques suffisamment solides pour ne plus être récusée et avoir un pouvoir explicatif convaincant (voir dans Cenoz/Gorter 2011). On voit dans Coste et al. (1997) qu'elle est définie comme la compétence à communiquer et interagir d'un individu maîtrisant un certain nombre de langues à des niveaux différents et possédant l'expérience de plusieurs cultures. Cette compétence n'est pas caractérisée par la ressemblance entre les compétences mais par la différentiation entre des éléments distincts d'une compétence globale. Ceci conduit à l'acceptation de la nature partielle et déséquilibrée des compétences langagières des locuteurs plurilingues.

Selon Dewaele/Pavlenko (2003), cette compétence est un système complexe, dynamique et évolutif qui établit des liens entre les compétences d'un individu et les contextes où ces compétences s'appliquent, les différentes habiletés langagières d'un individu au sein de cette multicompétence, et entre cette multicompétence et les situations d'emploi.

Dans le cas des langues, la complexité implique une grande diversité de facteurs: la psychologie humaine, la nature des mécanismes de perception, l'effet des principes pragmatiques, le rôle de l'interaction sociale dans la communication, le caractère des mécanismes d'apprentissage, et donc les phénomènes d'identités et d'attitudes. Cette diversité conduit à adopter une perspective dite «émique » et donc à chercher un compromis méthodologique 
entre le qualitatif et le quantitatif (voir dans Cenoz/Gorter 2011) et à relativiser les regards et les positionnements.

\section{L'acquisition d'une troisième langue et le plurilinguisme}

En recherche en acquisition des langues (RAL), les regards se sont portés de la langue sur l'apprenant, l'interaction et son contexte et sur les processus. L'acquisition est un système complexe, dynamique où les variables interagissent et s'influencent mutuellement (de Bot et al. 2007). Le contexte influence la langue en raison des modifications qu'il impose au code (Larsen-Freeman/Cameron 2008). L'émergentisme envisage la langue en tant que système de processus issu de l'interaction entre des processus biologiques et environnementaux. Il postule des principes provenant des systèmes où le rôle de la temporalité des événements exerce un impact considérable sur les résultats et les développements et où l'interaction entre les composants est vue comme provoquant un changement global, pouvant être considéré comme inédit, irréductible et imprévisible.

Les approches socioculturelles et écologiques jouent également un rôle important dans la RAL de façon compatible avec l'émergentisme ou la Théorie des systèmes dynamiques (Dynamic Systems Theory, DST) (van Lier 2004), ce qui rejoint ce qui a été dit ci-dessus concernant une approche à la fois 'émique' et 'éthique'.

La question qui se pose aujourd'hui, consiste à déterminer si la langue est traitée selon un module spécifique ou non, ce qui d'après Randall (2007) se confirme au niveau conceptuel et processuel, avec des séparations au niveau des formes. On peut penser que le contenu conceptuel nécessite peut-être une réorganisation permanente pour l'accommodation des concepts ou de leurs organisations puisque les contextes culturels les voient différemment.

\section{Les modèles plurilingues}

Le modèle dynamique du plurilinguisme (Herdina/Jessner 2006) décrit un développement de la langue caractérisé par six facteurs: la non-linéarité, la réversibilité, la stabilité du système langagier, l'interdépendance, la complexité et le changement qualitatif. Les changements linguistiques d'un individu avec le temps, mais aussi l'interaction de facteurs variés conduisent à la complexité et au caractère autodynamique du modèle. Ces facteurs comprennent de même: l'attitude plurilingue et les compétences métalangagières, le processus d'acquisition d'une langue, la motivation, la compétence perçue pour la langue, la confiance en soi, l'anxiété de la langue dans un système plurilingue (caractéristiques émique et éthique). Williams/Hammarberg (1998) ont proposé une distinction entre deux types de rôles joués par les langues/cultures 2 (L/C 2), lors de l'acquisition d'une L/C 3. Une langue active au cours du processus de communication en L3 peut jouer, soit un rôle instrumental en vue de gérer la 
situation communicative, soit un rôle fournisseur, c'est-à-dire offrant un matériau langagier pour la production orale en L/C 3. D'après Herdina/Jessner (2006), la distinction traditionnelle entre alternances codiques, pour ce qui est du bilinguisme, et transfert en RAL, a des justifications historiques. Toutefois, sur le plan méthodologique, elle n'est pas validée pour ce qui est de la recherche sur le transfert dans les systèmes linguistiques multiples. Une position holistique conduit à voir transfert, mélange de codes et alternance codique comme différentes positions sur un même continuum. Sans trop entrer dans le débat théorique, nous postulerons que le concept d'activation partielle d'une autre langue que celle attendue avec adaptation plus ou moins adéquate au fonctionnement de la langue cible est un concept opératoire pour une compréhension cohérente des phénomènes ${ }^{3}$.

Williams (2002: 29), affirme que « translanguaging ${ }^{4}$ is a natural way of developing and strengthening both languages whilst simultaneously gaining a deeper understanding ». C'est une façon d'établir des ponts entre les codes dans la communication quotidienne, scolaire ou sociale. Il reste à mesurer l'efficacité de ces pratiques sur l'acquisition. Enfin, selon Canagarajah (2007), le terme 'codemeshing' souligne la fluidité entre les codes qui sont traités comme les parties d'un système intégré.

Au-delà de notre adhésion au principe de non-modularité du cerveau, du postulat que toute production langagière est une modalité de l'intercompréhension et de l'alternance codique et que l'activation d'un code ne va pas de pair avec la désactivation des autres, nous garderons en mémoire que la multicompétence langagière n'est pas dissociable des contenus véhiculés dans des contextes variables.

On observe que la sociolinguistique considère les problèmes cognitifs, on parle même de sociolinguistique cognitive (Geeraerts et al. 2010). Ces regards croisés et pluriels répondent à la complexité des phénomènes plurilingues intimement liés à la pluralité des individus, des contextes et des activités sociales (tâches).

De cette situation est né le concept d'éducation plurilingue (emploi de deux ou plus de deux langues dans un contexte où l'objectif des écoles est le plurilinguisme et la multilittératie en lien avec la multimodalité. Dans Cenoz/Gorter (2011), on voit qu'un curriculum intégré offre les meilleures conditions pour développer une sensibilité métalinguistique qui renforce l'acquisition des différentes langues. Le plurilinguisme réceptif (intercompréhension des langues parentes par exemple) est perçu comme un moyen d'accroître la mobilité et la

\footnotetext{
${ }^{3}$ Narcy-Combes 2011 (conversation privée).

${ }^{4}$ Input dans une langue, tâche dans une autre.
} 
communication entre les Européens. Néanmoins une approche globale implique un grand degré de coordination entre tous les enseignants dans le cadre d'un curriculum intégré (Elorza/Muñoa 2008).

Il faudra vérifier que mélanges et changements de codes pour des besoins langagiers ou afin d'être plus créatifs contribuent de façon positive aux développements des identités plurilingues et pluriculturelles comme l'affirment Kramsch/Whiteside (2007).

On ne saurait ignorer les aspects que la multimodalité apporte au plurilinguisme (Kalantzis/Cope 2007). La technologie multimédia et des médias, dont le web 2.0, permet une littératie multimodale plus riche qu'auparavant (voir dans Cenoz/Gorter 2011). Avant de mesurer les effets de ces pratiques quand elles se combinent, nous allons aborder les notions de CLIL, de connaissance distribuée et de dispositif qui complèteront notre réflexion.

\subsection{Enseignement « bilingue » (langage/contenu)}

A la suite de Gajo (2006), nous définirons l'enseignement bi-/plurilingue comme la mise en place de dispositifs permettant des apprentissages disciplinaires et linguistiques par le recours total ou partiel à une L2 dans le cadre d'une ou de plusieurs disciplines non linguistiques (DNL). Cette définition semble implicitement supposer que la L2 est en place, ou que les problèmes que pose l'acquisition de L2 sont traités à part. En ce qui concerne cet enseignement, selon Cummins (1994), l'école doit pouvoir soutenir le développement langagier des apprenants en transformant leur répertoire langagier, fonctionnel du point de vue de la communication quotidienne et de la socialisation première (Basic intercommunication skills ou BICS) en un répertoire qui permet le développement des fonctions cognitivo-langagières liées à la construction des savoirs disciplinaires en situation formelle (Cognitive academic language proficiency ou $C A L P)$. Les langues de scolarisation devraient donc être acquises avec une compétence $C A L P$. Les recherches sur l'éducation bilingue montrent que travailler des concepts en deux langues permet d'enrichir l'intention et l'extension de ce concept, mais il importe qu'une des deux langues soit familière à l'élève pour que cet effet bénéfique puisse opérer. Les études sur les apprentissages bilingues (immersion, EMILE ou CLIL) rappellent par ailleurs qu'un pidgin de classe se crée si on n'est pas attentif à la structuration de la langue (Dalton-Puffer 2007). Dans la mesure où nous œuvrons dans l'enseignement supérieur, nous nous sommes penchés sur les effets de ce type d'enseignement en Europe. Dans Smit/Dafouz (2013), on perçoit un certain nombre de points faibles que nous avons cherché à éviter dans nos formations. 
- En France, l'obstacle principal vient probablement de la perception que les enseignants disciplinaires ont de leurs compétences langagières, en particulier en production.

- Les enseignants ont des cultures professionnelles, et donc des stratégies pédagogiques différentes, et leur insécurité n'est pas la même. Les travaux écrits et les présentations orales sont interprétés différemment selon les disciplines et les enseignants.

- Les retours sur la langue ne font pas partie des préoccupations des experts en contenu, ce qui n'est pas sans effets.

Il est nécessaire de prendre en compte et d'enseigner la cohésion et la cohérence des discours scientifiques et académiques. Si les étudiants n'ont pas la possibilité de se voir offrir une formation en langue spécifique, il y a peu de chances pour que les objectifs linguistiques puissent être atteints. La CALP (voir plus haut) est toujours implicitement considérée comme allant de soi, alors que ce n'est pas le cas.

\subsection{Positionnement sur la connaissance distribuée et la co- construction des connaissances}

Connaissance distribuée et co-construction des connaissances sont issus d'une théorisation psychologique développée initialement par Hutchins (1995), qui, inspiré par la sociologie, les sciences de la cognition et les théories de Vygotski (1934-1997), postule que le savoir résulte d'une réflexion et d'un travail coordonnés entre humains, artefacts et environnement.

Le savoir humain et la cognition sont distribués entre les individus, les objets et les supports qu'offre notre environnement. De ce fait, les processus de construction de savoir sont également distribués entre les individus, entre le mental et le physique et le social, et dans une relation où le temps joue un rôle. Les humains pensent en collaboration avec d'autres et grâce à des outils et dispositifs fournis par le contexte (Salomon 1997). Dans notre domaine, la Communication Médiée par Ordinateur s'est beaucoup appuyée sur ce concept et nous a ouvert beaucoup de pistes (Bertin et al. 2010; Lamy/Hampel 2007; Mangenot 2001; White 2003, etc.). Ce concept nous a paru crucial comme le montreront les projets qui vont suivre.

\section{Synthèse: les points qui ont guidé la mise en place des pro- jets}

Pour mettre en place les projets qui font l'objet de la seconde partie, nous nous sommes appuyés sur les points suivants: 
Le dispositif mis en place en fonction du contexte d'enseignement/apprentissage s'appuiera sur les technologies de l'information et de la communication (TIC) qui permettent la multicanalité utile dans notre domaine (son, image, texte) et sont sources de multiréférentialité (cf. Lancien 1998). Leur souplesse et leur adaptabilité facilitent la solution à un grand nombre de problèmes de terrain. Comme partie intégrante de la vie des publics auxquels le dispositif est proposé, elles sont aussi incontournables.

Les tâches tiendront compte de la relation entre discours, contenu, culture et permettront de répondre à la pluralité des individus, des contextes et des activités sociales.

Nous chercherons un compromis méthodologique entre le qualitatif et le quantitatif et relativiserons nos positionnements.

Les projets sont appuyés sur le concept d'éducation plurilingue. Ceci a conduit dans les différents exemples à l'adoption d'un curriculum intégré impliquant plusieurs langues, ce qui a amené les enseignants à coordonner leurs actions.

Nous avons cherché à vérifier que les mélanges et changements de codes pour des besoins langagiers ou afin d'être plus créatifs contribuent de façon positive aux développements des identités plurilingues et pluriculturelles des individus (Kramsch/Whiteside 2007).

\section{$3 \quad$ Projets}

Nous allons présenter maintenant quatre projets différents mais qui sont la déclinaison du même concept adaptable en fonction des contextes, de contraintes et des attentes.

\subsection{Master 2 à distance (Paris 3-DFLE): Dispositifs et TIC: de la classe au distanciel}

Ce module est décrit dans les détails, ce qui permettra de comprendre son architecture et son fonctionnement et, comme il apparaitra plus bas, de comprendre plus vite en quoi les autres modules ressemblent ou diffèrent de celui-ci.

\section{Objectifs:}

- Construire le concept de dispositif conçu comme un cadre organisateur. Construire le cadre (théories et méthodologie), et les concepts liés aux TIC et à la Formation Ouverte et à Distance (FOAD). 
- Exploiter les ressources existantes dans différents contextes d'apprentissage. Apprendre à ébaucher une maquette pertinente dans un contexte donné pour un public cible précis.

\section{Organisation:}

- Les outils à disposition des étudiants, le forum et les documents, permettent que même à distance se crée un esprit de 'corps' et que la coopération facilite le travail. Le tuteur répond aux questions des étudiants au fur et à mesure qu'elles sont formulées.

\section{Quatre thèmes ${ }^{5}$ :}

- Dix articles scientifiques en français permettent de construire une connaissance sur chaque thème. Les étudiants recherchent les données sur chaque thème et construisent leur connaissance. La collaboration (forum) permet de compléter l'information, d'échanger questions et réponses sur les problèmes rencontrés. L'enseignant y participe.

- Les étudiants sont invités à partager des informations au fur et à mesure que des données sont recueillies pour faciliter le repérage des uns et des autres dans les documents et à poser des questions autant pratiques que théoriques et à y répondre.

La synthèse en deux pages du travail sur chaque thème est personnelle. Elle reflète l'état de la compréhension du thème. L'analyse d'un dispositif est aussi un travail plus personnalisé. Selon le calendrier, les étudiants envoient leurs synthèses à l'enseignant qui fournit une rétroaction personnalisée. Un échange personnel a eu lieu spontanément par mail pour des remerciements et demandes de précisions (socialisation/affects).

\section{Critères pris en compte pour le travail}

- Les lectures et la participation à des débats sur forum permettent de moduler la note.

- Rédaction de quatre notes de synthèse personnelle ( 2 pages avec nombre de mots).

- Analyse d'un dispositif (cinquième note), un modèle de grille est fourni.

5 1) Acquisition / production langagière/caractéristiques individuelles, 2) De la classe de langue à d'autres formes de regroupement, 3) Une approche par tâches, 4) Apport des TIC: un regard critique. 


\subsection{Maroc (Université Hassan II - Mohammedia)}

Cette formation s'adresse à des étudiants en master 1 de didactique des langues qui se destinent à l'enseignement du français au Maroc.

Ce dispositif est essentiellement à distance, avec un séminaire de trois jours en présentiel en fin de parcours (ce séminaire, avec une visioconférence à miparcours, est la différence majeure avec le module précédent. Ces deux éléments créent un lien socio-affectif important).

Un forum est ouvert, pas sur une plateforme mais sur une liste commune, ce qui le rend moins efficace. Le séminaire en présence des deux enseignants permet un cadrage final, des échanges, et une évaluation sommative correspondant à l'application des théories rencontrées.

\subsection{Nantes (LEA-Faculté des Langues)}

Deux projets plurilingues en Master 2 LEA responsable logistique International. Cette formation pilote s'adresse à des étudiants de Master 2 Langues Etrangères Appliquées qui étudient à un haut niveau une autre langue en plus de l'anglais. L'objectif est de réunir les conditions qui permettraient de dépasser le cloisonnement de l'enseignement des langues à l'université, de se rapprocher des conditions d'emploi en situation professionnelle, et donc de les inciter à s'appuyer sur les répertoires multiples à leur disposition.

\subsubsection{Premier projet: la revue de presse multilingue}

En début d'année, 5 groupes de 4 étudiants sont constitués de façon à chaque groupe rassemble quatre langues (français et l'anglais commun à tous).

Les étudiants sont invités à choisir une thématique logistique dans une liste de 15 et à réfléchir à une question de recherche à partir de ces thématiques. Ils ont trois mois pour collecter des articles, académiques et managériaux, récents, complémentaires et contradictoires, en français, anglais, allemand, espagnol, chinois, selon les groupes. Un suivi est assuré sur une plate-forme Moodle et/ou en présentiel par une équipe de cinq enseignants en fonction des compétences de chacun.

$\mathrm{Au}$ terme du projet, les étudiants remettent le résultat de leurs travaux sous forme de rapport: 
- 2 pages de synthèse (réponses aux questions) en 4 langues en fonction des groupes,

- 10 pages de revue de presse (développant les réponses et prenant appui sur les articles collectés) rédigées en anglais et italien, ou allemand, ou espagnol suivant les groupes,

- la bibliographie des articles collectés et, en annexe, une copie des articles.

Le projet se termine par une présentation orale de la revue de presse, en langue anglaise et en présence de professionnels de la logistique.

\subsubsection{Projet Lexlogil}

Parallèlement, en réponse à un besoin des professionnels de la logistique, les étudiants contribuent à la constitution d'un lexique plurilingue de la logistique. Les termes et expressions sont en lien avec la thématique retenue pour la revue de presse. Au début du semestre, chaque étudiant reçoit un terme en français, à partir duquel il rédige une fiche en français, une en anglais et une en LV2, selon le schéma complexe qui les amène à rédiger trois fiches en trois langues sur le même terme. Sont à la disposition des étudiants:

- un forum pour le partage de références bibliographiques ou de sitographiques intéressantes et pour la création de bibliographies et sitographies personnelles,

- un forum d'entraide pour les problèmes informatiques, techniques ou linguistiques.

Le suivi est assuré par la même équipe d'enseignants, à distance. Les fiches sont remises en fin de parcours. Un retour linguistique est donné par les enseignants de langues, et le contenu est validé par des professionnels. Chaque fiche comprend le terme en français, sa traduction, sa catégorie grammaticale, la définition, des exemples d'emploi en contexte, des notes linguistiques éventuelles, la date de création et le nom de l'auteur et enfin les sources, avec des renvois à des documents graphiques ou vidéo. L'objectif est à terme d'élaborer un outil suffisamment complet pour répondre à la demande des entreprises de logistique. 


\subsection{Projet Siegen-Paris: « Technologies de l'Information et de la communication [TIC] et enseignement des langues » (ins- piré du module à distance de Paris 3 ci-dessus)}

Une unité d'enseignement (Master 2 Professionnel) hybride commune aux Universités de Siegen et Paris 3 a été conçue par Dagmar Abendroth-Timmer, Jean-Paul Narcy-Combes et Jose Aguilar, avec appui sur la plateforme Moodle de l'Université de Siegen.

Il s'agit d'un module pilote qui réunit 21 (11 à Paris et 10 à Siegen), étudiants volontaires, mais notés à la fin du semestre (Paris 3: 7 francophones natifs, 4 locuteurs natifs d'autres langues et Siegen: 9 germanophones natifs, 1 hellénophone native). Le travail en tandems est organisé par les tuteurs qui gèrent la plateforme et les utilisateurs, assurent la médiation pédagogique des 10 tandems franco-allemands, les aspects techniques et l'évaluation.

Le travail à fournir est semblable à celui des modules de Paris 3 ou du Maroc (cinq tâches par tandem à soumettre aux tuteurs selon un calendrier). Le corpus de 30 articles scientifiques issus de revues internationales est plus conséquent que dans le module à distance de Paris 3 (acquisition, didactique des langues et TIC, en allemand, anglais, espagnol et français).

La production des 5 tâches par les tandems leur imposait de:

- Choisir 3-5 articles (corpus), trouver de nouveaux articles,

- Négocier le partage des tâches de lecture,

- Sélectionner les idées à conserver dans chaque article, structurer le plan.

Les négociations se faisaient à distance (forums, chat, Moodle, Skype) et les échanges étaient soumis aux tuteurs (via Moodle). Chaque tandem avait le choix a) de la langue b) des modalités de rédaction de la synthèse c) de la fréquence/modalité des rencontres avec le partenaire. La seule consigne était de rédiger la synthèse. La participation des étudiants aux visioconférences n'était pas obligatoire.

\subsection{Résultats et analyse}

L'interculturalité est une dimension réelle et concrète dans deux des projets sur quatre (Nantes et Siegen-Paris). En ce qui concerne le projet Maroc, elle a certainement agit sur la relation entre les tuteurs et les étudiants. Le module à distance de Paris 3 sert de groupe témoin sur ce point, la construction du savoir y est la plus efficace, mais les effets de contexte sont tels que nous ne pouvons pas affirmer que cela confirme certaines recherches. 
Dans les quatre modules, la participation des apprenants est remarquable, dans le module à distance le taux d'abandon est quasi nul, ce qui surprend dans cette formation.

Les tuteurs ont noté la richesse des échanges, la multiplicité de facettes à partir desquelles ces échanges ont été construits, et le fort engagement des participants, qui a été moins évident sur le forum avec le Maroc (pas un vrai forum, mais un échange de mails sur une liste). Ceci confirme que l'organisation matérielle et technologique est cruciale.

L'espace virtuel est un milieu qui favorise un débat légitime sur diverses questions (académiques, pédagogiques, culturelles, techniques, personnelles, ...) sans trahir le caractère institutionnel, et l'asynchronie joue un rôle très positif au niveau de la construction des connaissances en évitant la pression communicative des échanges en présence.

Les TIC jouent un rôle clé et permettent un accès aux multilittéracies, et un enrichissement collectif, tout en favorisant le développement personnel de chacun.

L'examen des rôles de l'enseignant confirme les écrits sur le travail hybride et à distance (Bertin et al 2010; Lamy/Hampel 2007; White 2003). Ils sont tour à tour tuteurs et médiateurs (dans le cadre des tandems et pour la technologie), organisateurs (pour les contenus, le calendrier, l'animation), régulateurs (de la situation d'apprentissage prise en charge par les étudiants), fournisseurs de savoir (dans le choix des documents).

Dans le contexte international (Siegen-Paris et Maroc), cela impose une négociation préalable très précise selon les cultures d'enseignement, en particulier au niveau de l'évaluation.

En ce qui concerne les points positifs (communs ou non) selon les étudiants:

Les projets montrent des points convergents (ou pas): le mode de travail par la réalisation de projets change le mode de relation entre les tuteurs et les étudiants (pas de dimension autorité-sanction). L'enseignant joue un rôle d'animateur, sauf en visioconférence où le rapport demeure plus traditionnel. Les TIC apportent un plus indéniable sur le plan de l'accès aux informations, aux langues différentes, à l'établissement de rapports plus 'visuels', même si dans certains cas (mais pas toujours) elles ont dû faire l'objet d'un apprentissage spécifique. Elles ne sont pas un obstacle, au contraire, à l'établissement de relations authentiques.

La collaboration entre pairs est effective dans tous les cas mais jouait sans doute un rôle plus grand dans le projet Siegen-Paris qui, plus que les autres, suscitait le besoin de négociation, des efforts de compréhension, et le besoin de la médiation enseignante dans un climat de confiance général. L'apprentissage 
par la réalisation d'un projet donne des résultats positifs. A cela s'ajoute une double dimension formative: une métaformation combinée à une formation en langue.

\section{Points négatifs (communs ou pas):}

Les limites observées concernent tout d'abord l'utilisation des forums qui doivent avoir un fonctionnement efficace: il manque un forum commun pour le projet Siegen-Paris, mais d'autres forums posent problème. Celui du Maroc est lent à démarrer et les étudiants sont loin d'en utiliser le potentiel, les étudiants de Nantes le boudent (mais ils créent des forums parallèles sur les réseaux sociaux, pour traiter des mêmes questions!), celui de Paris 3 a été très efficace.

Le travail en tandem ou en groupe est enrichissant mais complexe. Il génère parfois des résistances au niveau de l'évaluation, l'harmonisation et l'objectivité semblent difficiles à réaliser, mais il semble que ce problème ne soit pas uniquement lié à ce type de dispositif.

\section{Discussion et conclusion}

Les expériences ont pu être menées à terme à la satisfaction de tous, ce qui est logique dans la mesure où elles ont été mises en place par des enseignant-chercheurs qui se sont appuyés sur les résultats de la recherche. Les effectifs limités expliquent aussi la réussite.

Les résistances sont venues, sans surprise, des problèmes interpersonnels et interculturels que suscitent toute interaction, collaboration ou échange, et, sur un autre plan, de l'investissement accru que réclament ces organisations à la fois de la part des étudiants et de celle des enseignants. Si les étudiants s'investissent davantage, le travail de suivi est exigeant, et les tandems sont plus lourds à gérer à distance que des individus isolés.

Des résultats remarquables, supérieurs aux résultats obtenus en présentiel, ont été obtenus au niveau du savoir dans les modules nationaux, et solides dans les modules internationaux, ce qui correspond aux résultats décrits dans nos sources. Introduire le plurilinguisme sans le transnational est moins efficace (cas de Nantes), mais introduire le plurilinguisme et le transnational impose une coordination complexe et lourde difficilement applicable avec des effectifs lourds.

Les dispositifs ont évité, par contre, les faiblesses des dispositifs DNL traditionnels car le domaine était celui des enseignants de langues (sciences du langage), sauf à Nantes (domaine: logistique) où les différences de cultures professionnelles, et donc de stratégies pédagogiques, et de ce fait d'insécurité, ont été ressenties, bien que les spécialistes de logistique y soient à l'aise en anglais. En ce qui concerne les travaux écrits et les présentations, à Nantes, les spécia- 
listes en contenu étaient très sévères sur la qualité technique, mais acceptaient un anglais qu'ils pouvaient comprendre. Les spécialistes de langue étaient plus au fait de la langue, quoique tolérants à l'oral, mais acceptaient un exposé structuré et intéressant pour eux.

Il n'y a pas eu de problèmes sérieux de retours sur la langue, les étudiants avaient un niveau avancé et le géraient entre eux dans le projet Siegen-Paris. C'était également le cas à Nantes, où l'entrée en Master 2 est conditionné au niveau en langue, un peu moins avec le Maroc.

Il est néanmoins nécessaire de prendre en compte et de valider la cohésion et la cohérence des discours scientifiques et académiques (c'est le rôle du tuteur dans ses retours). En ce qui concerne ces discours, la $C A L P$ (voir plus haut) est toujours implicitement considérée comme allant de soi, alors que ce n'est pas le cas comme l'a montré le dispositif marocain où certains étudiants avaient du mal à formuler leurs interventions dans une langue structurée avec cohérence et dans un discours adapté.

En conclusion, ces expériences confirment la faisabilité de tels projets, leur pertinence et leur attractivité, mais également la complexité de leur fonctionnement. Généraliser à des promotions entières ce type de formation semble encore prématuré, il convient, de ce fait, de procéder à d'autres recherches-actions. Par contre, leur potentiel est très grand et ils paraissent une solution séduisante à la construction d'un enseignement européen intégré.

\section{Bibliographie}

Bertin, Jean-Claude / Gravé, Patrick / Narcy-Combes, Jean-Paul (2010). Second language distance learning and Teaching: theoretical perspectives and didactic ergonomics. Hershey (Penn.): IGI Global.

Canagarajah, Athelstan Suresha (2007). Lingua franca English, multilingual communities, and language acquisition. Modern Language Journal 91: 921-937.

Cenoz, Jasone / Gorter, Durk (2011). A Holistic Approach in Multilingual Education: Introduction. Special issue Toward a Multilingual Approach in the Study of Multilingualism in School Contexts. The Modern Language Journal 95: 3.

Coste, Daniel / Moore, Danièle / Zarate, Geneviève (1997). Compétence plurilingue et pluriculturelle. Strasbourg: Conseil de l'Europe.

Cummins, Jim (1994). Knowledge, power, and identity in teaching English as a second language. In: Genesee, Fred (éd.): Educating second language children: The whole child, the whole curriculum, the whole community. Cambridge: CUP. 103-125.

Dalton-Puffer, Christiane (2007). Discourse in Content and Language Integrated Learning (CLIL) classrooms. Amsterdam-Philadelphia: John Benjamins. 
Dewaele, Jean-Marc / Pavlenko, Aneta (2003). Productivity and lexical diversity in native and non-native speech: A study of cross-cultural effects. In: Cook, Vivian J. (éd.): Effects of the second language on the first. Clevedon, England: Multilingual Matters. 120-141.

de Bot, Kees / Lowie, Wander / Verspoor, Marjolijn (2007). A dynamic systems theory approach to second language acquisition. Bilingualism 10: 7-55.

Elorza, Itziar / Muñoa, Inmaculada (2008). Promoting the minority language through integrated plurilingual language planning: The case of the ikastolas. Language, Culture and Curriculum 21: 85-101.

Gajo, Laurent (2006). Types de savoir dans l'enseignement bilingue: problématicité, opacité, densité. Education et sociétés plurilingues. Juin 2006: 75-87.

Geeraerts, Dirk / Kristiansen, Gitte / Peirsman, Yves (éds.) (2010). Advances in cognitive sociolinguistics. Berlin: de Gruyter Mouton.

Herdina, Philip / Jessner, Ulrike (2006). A Dynamic Model of Multilingualism, Perspectives of Change in Psycholinguistics. Clevedon: Multilingual Matters.

Hutchins, Edwin (1995). Cognition in the Wild. Cambridge, MA: MIT Press.

Kalantzis, Mary / Cope, Bill (2007). Language education and multiliteracies. In: May, Stephen / Hornberger, Nancy H. / Creese, Angela / Martin, Peter / Duff, Patricia (eds.): Encyclopedia of language and education: Vol.1: Language policy and political issues in education. New York: Springer. 195-211.

Kramsch, Claire / Whiteside, Anne (2007). Three fundamental concepts in SLA and their relevance in multilingual contexts. Modern Language Journal 91: 905-920.

Lancien, Thierry (1998). Le multimédia. Paris: Clé international.

Lamy, Marie-Noëlle / Hampel, Régine (2007). Online Communication in Language Learning and Teaching. New York: Palgrave.

Larsen-Freeman, Diane / Cameron, Lynne (2008). Complex systems and applied linguistics. Oxford: Oxford University Press.

Mangenot, François (éd.) (2001). Interactivité, interactions et multimédia. Notions en Questions. $\mathrm{n}^{\circ}$ 5, Lyon: ENS Editions.

Narcy-Combes, Jean-Paul (2005). Didactique des langues et TIC, vers une recherche-action responsable. Paris: Ophrys.

Piaget, Jean (1970). Psychologie et épistémologie. Paris: Gonthiers Denoël.

Randall, Mick (2007). Memory, Psychology and Second Language Learning. AmsterdamPhiladelphia: John Benjamins.

Salomon, Gavriel (1997). Distributed cognitions: Psychological and educational considerations. Cambridge: Cambridge University Press.

Simondon, Georges (1989). L'individuation psychique et collective. Paris: Aubier.

Smit, Ute / Dafouz, Emma (2013). AILA Review, Vol 25. Integrating Content and Language in Higher Education. Amsterdam/Philadelphia: John Benjamins.

van Lier, Leo (2004). The ecology and semiotics of language learning: A sociocultural perspective. Boston: Kluwer Academic. 
Vygotski, Lev (1997). Pensée et langage. 3ème édition. Paris: La Dispute.

White, Cynthia (2003). Language Learning in Distance Education. Cambridge: CUP.

Williams, Cen (2002). Extending bilingualism in the education system. Education and lifelong learning committee ELL-06-02. Online: http://www.assemblywales.org/3c91c7af 00023d820000595000000000.pdf (dernier accès [13.03.2013]).

Williams, Sarah / Hammarberg, Björn (1998). Language switches in L3 production: Implications for polyglot speaking model. Applied Linguistics 19: 295-333. 


\title{
Ein Beitrag zur Sensibilisierung für eine Mehrsprachigkeitsdidaktik in der Lehrerbildung
}

\author{
Anja Wildemann / Mahzad Hoodgarzadeh / Olga Esteve / \\ Rebecca Walter (Koblenz-Landau, Barcelona)
}

Throughout the two-year EU project MuViT (Multiliteracy Virtual), in addition to the language software for pupils, a Teacher Education Curriculum for pre- and in-service teacher training has been developed. This new tool is based on the principles of the multiliteracy pedagogic approach and reflexive learning derived from experiences. Prospective or already active language teachers get the opportunity to expand their technical and pedagogical knowledge as well as their repertoire in the area of multilingual multi-media teaching. The fundamental reference points of such teacher education are the personal experiences and attitudes of the teacher students, which will be analyzed and, if necessary, redirected during all training phases.

A lo largo de los dos años de duración del proyecto MuViT (Multiliteracy Virtual), aparte del desarrollo de un software específico para el alumnado, se desarrolló un Currículum para la Formación inicial y Continua del Profesorado. El enfoque adoptado se basa en los principios de la multiliteracidad y del aprendizaje reflexivo y experiencial. El Currículum ofrece a futuros profesores o a profesores ya en activo la oportunidad de ampliar su conocimiento tecnológico y pedagógico así como sus estrategias en el área de la enseñanza multilingüe y multimodal. Los elementos clave que fundamentan dicho currículum son las experiencias personales y las propias actitudes que serán objeto de análisis y, si es necesario, de redirección a lo largo de las fases del programa de formación.

\section{$1 \quad$ Einleitung}

Unterricht, der Lernende auf eine aktive und kritische Teilnahme in unserer medialen, mehrsprachigen und transkulturellen Gesellschaft vorbereitet, fördert die Entwicklung multiliteraler Kompetenzen (MuViT Group 2011).

Die Förderung multiliteraler Kompetenzen als Anliegen eines Sprachunterrichts, der die gesellschaftlichen und individuellen Veränderungen aufgreift, setzt nicht nur die frühen konzeptionellen Überlegungen der New London Group (1996) um, sondern implementiert darüber hinaus Aspekte des Language AwarenessAnsatzes (vgl. Hawkins 1984; Luchtenberg 2010). In Anlehnung daran wurde innerhalb des MuViT-Projektes (siehe auch den Beitrag von Elsner in diesem Band) ein Curriculum für die Lehrerfort- und -ausbildung entwickelt. Es zielt darauf ab, sowohl fachliches und fachdidaktisches Wissen zu vermitteln als auch eine Veränderung der Einstellung gegenüber Mehrsprachigkeit langfristig zu erwirken. Ausgangspunkt ist die Tatsache, dass in mehrsprachigen Lehr-LernKontexten mindestens zwei Sprachen eine Rolle spielen, wir es aber nicht selten mit einem Konglomerat aus diversen Erst-, Zweit- (je nach Aufenthaltsort) und 
modernen Fremdsprachen $\mathrm{zu}$ tun haben. Für diese heterogenen Formen vorhandener Mehrsprachigkeit gibt es zwar bereits Vorschläge für sprachensensible Unterrichtskonzepte (vgl. Krumm 2010; Neuner 2003; Oomen-Welke 1999, 2010; Wildemann 2010), jedoch finden sie sich kaum in der Unterrichtsrealität wieder. Eine Erklärung dafür ist die Tatsache, dass unter Sprachlehrkräften nach wie vor Wissens- und Kompetenzlücken vorhanden sind, die zumeist auf eine fehlende oder unzureichende Qualifizierung für den Unterricht in mehrsprachigen Lerngruppen zurückzuführen sind. Die Divergenz von Forderungen der Europäischen Kommission nach einer europäischen Mehrsprachigkeit (vgl. Europäische Kommission 2008: 5) und schulischer Sprachhegemonie ist eine unmittelbare Folge dieses Ausbildungsdesiderates (vgl. Hansen 2010). Eine weitere Konsequenz einer fehlenden Qualifizierung offenbart sich nicht selten in den Einstellungen von Lehrerinnen und Lehrern gegenüber Mehrsprachigkeit (vgl. Cantone 2011; Pünter 2012). Besonders bedenkenswert ist dabei die Erkenntnis, dass sich subjektive Theorien häufig schon frühzeitig verfestigen und nur schwer veränderbar sind (vgl. Blömeke 2005; Rank 2012). Eine gelingende Lehrerbildung muss daher bereits im Studium nachhaltig an den subjektiven Theorien der angehenden Lehrkräfte ansetzen und das Gelernte mit den eigenen Erfahrungen und Einstellungen koppeln. Nur wenn Lehrkräfte im Rahmen von Fortbildungen erleben, dass sie ihr dort neu erworbenes Wissen in der Unterrichtspraxis erfolgreich anwenden können, sind sie bereit, ihre Einstellungen entsprechend zu überdenken und anzupassen. Aus diesem Grund basiert das hier vorgestellte MuViT Teacher Education Curriculum (vgl. MuViT Group 2012) auf Ansätzen des reflexiven Erfahrungslernens und geht spiralcurricular von den Unterrichtserfahrungen, Vorstellungen und Präkonzepten zukünftiger und bereits tätiger Lehrkräfte aus. Primäres Ziel ist es, Lehrkräfte für eine Mehrsprachigkeitsdidaktik zu sensibilisieren und sie mit konkreten Formen der Umsetzung vertraut zu machen (vgl. Esteve 2010; Wildemann 2010, 2011).

\section{Prinzipien des MuViT Teacher Education Modells}

\subsection{Reflexives Erfahrungslernen als Ausgangspunkt}

Bisherige Forschungsergebnisse zur Professionalisierung von (angehenden) Lehrkräften zeigen uns, dass Teacher Education Konzepte, welche auf der Transmission von theoretischen Kenntnissen basieren, nur geringfügige qualitative Veränderungen in der eigenen Unterrichtspraxis zur Folge haben (vgl. Korthagen 2001; Lankes 2008; Lipowsky 2011; Schumann/Eberle 2009). Ein Grund dafür scheint die zu geringe Verbindung zwischen Theorie und praktischer Realisierung zu sein. Die unmittelbare Folge daraus ist, dass es Lehrkräften häufig nicht gelingt, das in der eigenen Unterrichtspraxis Wahrgenommene mit dem erworbenen fachlichen und fachdidaktischen Wissen zu verknüp- 
fen und schließlich anzuwenden. Eine erfolgreiche Lehrerbildung bedarf daher einer fortwährenden Verzahnung von subjektiver und schulischer Ausgangssituation, fachlichem und fachdidaktischem Input sowie der konkreten Anwendung in der eigenen Unterrichtspraxis. Diesbezüglich schlägt das reflexive Erfahrungslernen eine Brücke zwischen Theorie und Praxis und bietet daher eine gute Grundlage für eine nachhaltige Lehrerbildung. Hierbei bilden die Erfahrungen jeder Lehrperson und die kritische Reflexion über ihre eigene Praxis den Ausgangspunkt. Das beinhaltet zugleich eine Berücksichtigung der Vorund Einstellungen, welche Ziel des reflexiven Erfahrungslernens sind und nach denen die Lehrperson sich bedeutsam aus- und fortbildet (vgl. Esteve et al. 2010), wenn:

- eine klare Verbindung zu den eigenen Erfahrungen, welche sowohl erlebte Lehr- und Lernsituation implizieren als auch die eigenen Vorstellungen darüber, was es bedeutet Sprachen zu lehren und lernen, geschaffen wird;

- ausgehend von dieser Bewusstmachung ein Reflexionsprozess bei den Lehrer/-innen gefördert wird, der auf den Aufbau eines signifikanten didaktischen Wissens im Bereich der Mehrsprachigkeitsdidaktik ausgerichtet ist;

- auf der Basis der Reflexionsprozesse die Lehrer/-innen mit neuen methodischen Verfahren konfrontiert und weiterhin dazu aufgefordert werden, die eigenen subjektiven Theorien neu zu durchdenken.

Nach dieser Auffassung wird der Aus- und Fortbildungsprozess global als ein kognitiver dreiphasiger Prozess verstanden, der ausgehend von der kritischen Analyse der eigenen Erfahrungen und subjektiven Theorien (Sensibilisierung) über eine darauf folgende kritische Auseinandersetzung mit neuen Inhalten und neuem Wissen (Kontrastphase) zur fundierten Neubeschreibung der eigenen Denk- und Handlungsweisen führen soll (Evaluation und Neubearbeitung).

\subsection{MuViT Teacher Education}

Das innerhalb des MuViT-Projektes entwickelte Teacher Education Curriculum baut auf dem Lehrerbildungsmodell des reflexiven Erfahrungslernens auf, erweitert es jedoch um drei Prinzipien, deren Legitimation mit der derzeitigen Ausgangssituation im Folgenden zu begründen ist. Bisher verfolgen europäische Schulsysteme oft eine sprachliche Homogenität (vgl. Holzbrecher 2011; Katsarou 2009) sowie eine eher traditionelle Lehre, die den methodischdidaktischen Einsatz des Computers im Unterricht kaum bis gar nicht beinhaltet (vgl. Frederking/Jonas 2008). Hierbei konzentriert sich der Sprachenunterricht zudem auf die „modernen“ Fremdsprachen und ignoriert zumeist andere Fremdund Herkunftssprachen, die die Schüler/-innen in unsere Klassenräume mitbringen. Lehrkräfte begründen dies häufig mit mangelndem Wissen über die Herkunftssprachen der Schüler/-innen. Eine Einbeziehung der Sprachen in den 
Unterricht erscheint für viele nicht möglich, weil sie selber nicht über die (vermeintlich) erforderlichen Sprachkompetenzen verfügen und die Sprachen nicht sprechen oder verstehen können (vgl. Elsner 2010). Des Weiteren machen Lehrkräfte darauf aufmerksam, dass sie sich nicht ausreichend für eine Integration und Nutzung von Computern im Unterricht ausgebildet fühlen und demzufolge Weiterbildungskurse benötigen (vgl. Grossmann 2008: 14; vgl. KIM-Studie 2006: 30ff., siehe auch KIM-Studie 2010). Für den Deutschunterricht kommen Frederking und Jonas zu vergleichbar alarmierenden Ergebnissen (vgl. Frederking/Jonas 2008) und attestieren den Lehrkräften gar eine „regressive Verweigerungshaltung“ (ebd.: 1). Ersichtlich wird an den genannten Daten die Erfordernis einer wirksamen Lehrerbildung. Lehrkräfte müssen sowohl für einen medienintegrativen als auch für einen mehrsprachigkeitsorientierten Sprachunterricht qualifiziert werden.

In Anlehnung an diese Überlegungen basiert das hier vorgestellte Teacher Education Modell auf den drei zentralen Prinzipien: kompetenzorientierte study outcomes, Prozess- sowie Praxisorientierung. Diese werden im Rahmen der Aus- bzw. Fortbildungsmodule aufs Engste miteinander verzahnt.

\subsubsection{Kompetenzorientierte study-outcomes}

Die study outcomes beschreiben, was Lehrkräfte am Ende der Aus- bzw. Fortbildung erlernt haben sollen. In Anlehnung an den Weinert'schen Kompetenzbegriff (vgl. Weinert 2001) betreffen die study outcomes nicht nur das neu erworbene Wissen, sondern auch die Anwendung erlernter Inhalte in unterschiedlichen Lehr-Lern-Konstellationen. Konkret heißt dies, dass Lehrkräfte die Fähigkeit zur Gestaltung und Durchführung eines für die eigene Unterrichtspraxis bedeutungsvollen, an Mehrsprachigkeit orientierten Sprachunterrichts erlangen sollen. Dazu bedarf es jedoch auf Seiten der Lehrkräfte entsprechender Kompetenzen, die sich in Bezug auf Einstellungen, Kenntnisse und Fähigkeiten wie folgt ausdifferenzieren lassen:

\section{Einstellungen:}

- Ein Bewusstsein über die sprachheterogene Zusammensetzung von Lerngruppen im Sprachunterricht entwickeln.

- Die Notwendigkeit, sprachliche Heterogenität bei der didaktischen Planung zu berücksichtigen und anzuerkennen.

- Eine Einstellung zu Interkultureller Erziehung und zum mehrsprachigen Lernen entwickeln. 


\section{Kenntnisse:}

- Ein Grundlagenwissen zu Mehrsprachigkeit, Language Awareness und Multiliteralität aufbauen.

- Kenntnisse über Erst- und Zweitspracherwerbsverläufe besitzen.

- Über Methodenkenntnis zum medienintegrativen Sprachunterricht verfügen.

\section{Fähigkeiten:}

- Erwerb grundlegender Fähigkeiten und Fertigkeiten, mit den heterogenen Spracherfahrungen von Schüler/-innen umzugehen.

- Kompetenz, sprachenförderliche Unterrichtssettings unter Einbeziehung verschiedener Medien zu realisieren.

- Fähigkeit, sprachliche Lernprozesse zu reflektieren und zu bewerten.

Primäres Anliegen des MuViT Teacher Education Ansatzes ist es, Lehrkräfte dafür zu sensibilisieren, Mehrsprachigkeit als Ausgangspunkt des sprachlichen Lernens zu verstehen. Deshalb ist jede Lehrkraft im Zuge der Fortbildung aufgefordert, ein eigenes, für ihre derzeitige Lehrsituation didaktisch passendes Unterrichtskonzept zu entwickeln. Es ist einerseits ihre Aufgabe, die verschiedenen multiliteralen Dimensionen des Lernens, wie funktionale, visuelle, digitale und multimodale Literalität (vgl. Wildemann 2010; Wildemann i. Dr.) $\mathrm{zu}$ integrieren. Andererseits sollen die sprachlichen Voraussetzungen der eigenen Schülerschaft berücksichtigt werden.

\subsubsection{Prozess- und Praxisorientierung}

Prozessorientierung in der Lehreraus- und -fortbildung zielt darauf ab, bei den Lehrkräften einen Entwicklungsprozess, der die Aneignung neuer didaktischmethodischer Kenntnisse beinhaltet, zu initiieren. Ein solcher Prozess ist nur dann wirksam, wenn er aus Sicht der Lehrkräfte als Bereicherung oder Erweiterung ihrer bisherigen Erfahrungen, Einstellungen und Kenntnisse verstanden wird (vgl. Esteve et al. 2010).

Im Zuge der Praxisorientierung werden die Lehrkräfte angeleitet, Umsetzungen für den eigenen Unterricht zu entwickeln, zu erproben und deren Ergebnisse zu reflektieren. Innerhalb der modularisierten Aus- oder Weiterbildung wird an Praxisbeispielen aufgezeigt, wie Unterrichtseinstiege und -verläufe gestaltet werden können. Darüber hinaus gibt es eine Praxisphase, in der die Lehrkräfte ihre Unterrichtskonzepte in ihren Klassen anwenden. Durch die reflexive Erprobungsphase wird die Umsetzung des erworbenen fachlichen und fachdidaktischen Wissens unmittelbar erfahrbar, und es findet eine bedeutsame 
Verzahnung von eigenen Erfahrungen und Kenntnissen mit dem neu Erlernten vor dem Hintergrund der aktuellen Lehrsituation statt.

\section{$3 \quad$ Modulares Lernen im Rahmen der MuViT-Lehrerbildung}

\subsection{Die MuViT-Pyramide}

Um den angeführten Prinzipien gerecht zu werden, wurde ein modulares Curriculum konzipiert, das auf die Befähigung der Lehrkräfte zur Gestaltung eines an Mehrsprachigkeit orientierten Sprachunterrichts abzielt. Da ein besonderes Augenmerk auf der Unterrichtsgestaltung liegt, die für die Lehrkraft in ihrer derzeitigen Situation realisierbar und daher bedeutsam ist, können die einzelnen Inhalte des Fortbildungscurriculums flexibel kombiniert, d. h. an die individuellen Bedürfnisse adaptiert werden. Insgesamt umfasst das MuViTCurriculum vier Module, wobei sich das Basismodul (Reflexives Lernen) durch die gesamte Qualifizierungsphase hindurchzieht und daher obligatorisch ist. Die weiteren Module beinhalten theoretische Grundlagen (Modul 1), eine Einführung in die MuViT Software (Modul 2) und schließlich das anwendungsorientierte Modul 3. Sie sind in der MuViT-Pyramide zusammengefasst. 


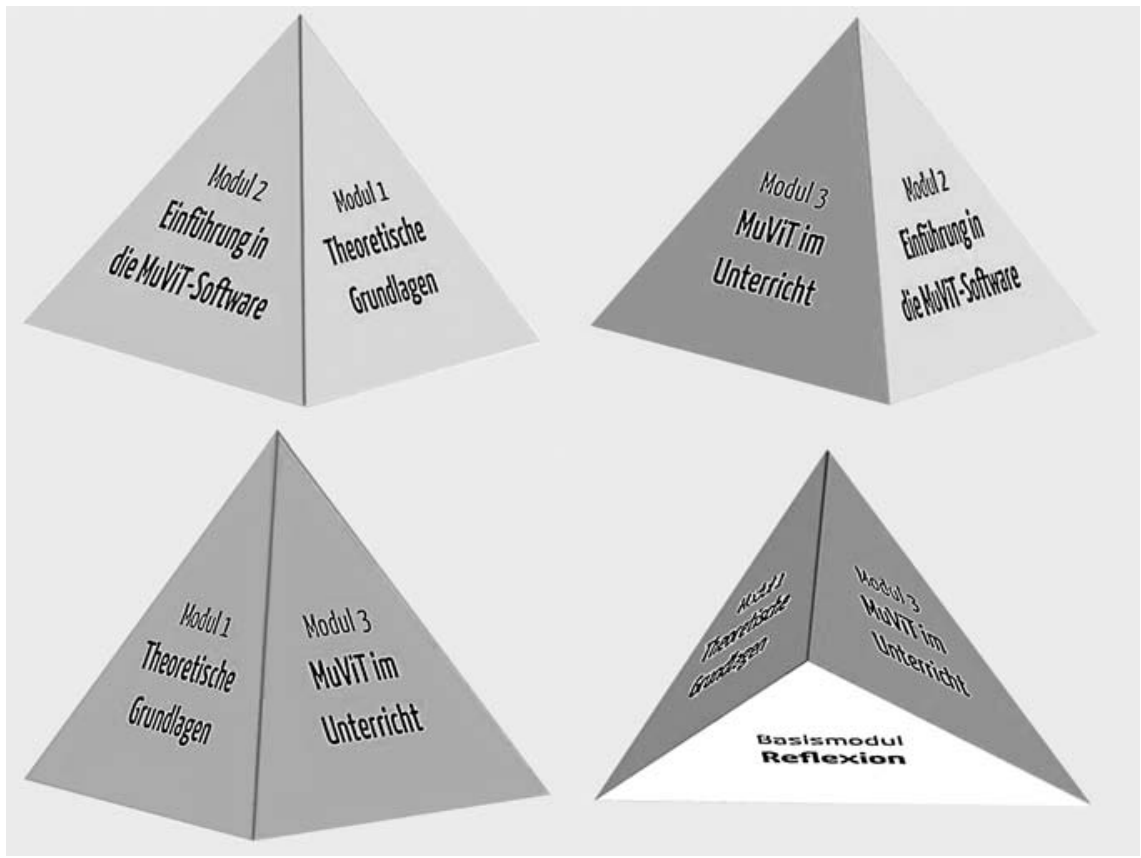

Abbildung 1: Die MuViT-Pyramide

\subsection{Das Basismodul: Reflexives Lernen}

In dem MuViT Teacher Education Curriculum ist das reflexive Lernen als Basismodul integraler Bestandteil der Qualifizierung. Es zielt darauf ab, den Lernprozess während der gesamten Dauer der Qualifizierung zu unterstützen und bildet das Fundament der MuViT-Pyramide (siehe Abb. 1). Es setzt sich aus drei Bausteinen zusammen, die den Phasen Sensibilisierung, Kontrast, Evaluierung und Neubearbeitung des oben beschriebenen Befähigungsprozesses entsprechen. Im ersten Baustein (Initialreflexion/Sensibilisierung) findet eine Analyse der eigenen Lehrpraxis bzw. der verinnerlichten Lehrkonzeption statt. Die Lehrkräfte werden dazu angeregt, gemeinsam mit anderen Lehrer/-innen über die eigenen Erfahrungen als Sprachlerner sowie über die Erfahrungen als Sprachlehrer im schulischen Sprachunterricht mit heterogenen Gruppen zu sprechen. Sie sollen somit sowohl die Lehrer- als auch die Lernerperspektive einnehmen. In den bisher im Rahmen des MuViT-Projektes durchgeführten Lehrerfortbildungen konnten wir feststellen, dass ein nicht unerheblicher Teil der Lehrkräfte bis dahin noch nie gezielt über seine subjektiven Theorien und Haltungen gesprochen hat. Folglich fehlte ihnen zumeist ein Bewusstsein dafür, 
welche Vorstellungen und Konzepte hinsichtlich eines mehrsprachigen Klassenzimmers sie verinnerlicht haben und unbewusst durch ihre Handlungen zum Ausdruck bringen. Die Form der Versprachlichung trägt somit dazu bei, dass Lehrkräfte sich ihrer Einstellungen bewusst werden und für einen reflexiven Lernprozess bereit sind. Die Versprachlichung zielt aber auch auf einen Perspektivenwechsel ab, in dessen Verlauf die Lehrer/-innen das sprachliche Lernen aus der Sicht der Lernenden nachvollziehen. Dazu eignet sich auch die Begegnung mit anderen, fremden Sprachen aus der MuViT Software.

Um die Lehrkräfte zum Nachdenken und Sprechen über ihre subjektiven Erfahrungen, Vorstellungen und Haltungen anzuregen, wurden folgende Reflexionsfragen entwickelt:

- Wie habe ich Sprachen gelernt?

- Wie ist die Situation in meinen/unseren mehrsprachigen Klassen?

- Welche Erfahrungen habe ich/haben wir mit solchen Klassen gesammelt?

- Wo liegen die Herausforderungen?

- Was verstehe ich unter einem mehrsprachigen Klassenzimmer?

Der zweite Baustein (Monitoring) findet während des weiteren Fortbildungsverlaufs statt und ist daher mit den theoretischen Inhalten verbunden, die im Modul II behandelt werden. Das Monitoring zielt auf zwei Aspekte ab: Erstens soll eine kritische Auseinandersetzung mit den theoretischen Grundlagen und ein Abgleich mit den eigenen Einstellungen erfolgen. Zweitens soll ausgehend von jener kritischen Reflexion der theoretischen Inhalte zu Mehrsprachigkeit, Language Awareness und Multiliteralität eine erste Umsetzung des bisher Erlernten stattfinden. Dies kann durch Handlungsvorschläge für den eigenen Unterricht und eine konkrete, auf die eigene Klasse abgestimmte Unterrichtsplanung geschehen. In dieser Phase werden Lehrkräfte ebenfalls durch Reflexionsfragen dazu angeregt, ihr Wissen und ihre Haltungen kritisch zu hinterfragen:

- Welche Ideen/Ansätze aus dem Gelernten sind für mich/meinen Unterricht/für meine Klasse relevant - und warum?

- Welche Ideen sind relevant für mich/uns in Bezug auf die Realisierung und Förderung von Mehrsprachigkeit im eigenen Unterricht? Was bedeutet dies für meinen/unseren Unterricht? 
Schließlich wird anhand des dritten Bausteins (Evaluation) der Transfer in die eigene Unterrichtspraxis und die kritische Analyse der eigenen Unterrichtsvorschläge sowie deren Erprobung angestrebt. Dieser Baustein ist mit den Zielen und Inhalten des dritten Moduls verbunden und beinhaltet die reflexive Auseinandersetzung mit der bisherigen Einstellung zur Mehrsprachigkeit. Die Reflexion wird durch folgende Fragen angeregt:

- Inwieweit hat sich meine eigene Einstellung zur Mehrsprachigkeit und meine Unterrichtskonzeption verändert?

- Was hat sich nach der Anwendung von MuViT an der Haltung der Schüler/ -innen geändert? Woran kann ich das feststellen?

- Wie findet jetzt die Sprachvermittlung zwischen den Schüler/-innen statt?

- Welche interkulturellen und mehrsprachigen Aushandlungsprozesse lassen sich beobachten?

- Was war für mich im gesamten Prozess wichtig?

Der begonnene reflexive Lernprozess endet nicht an dieser Stelle, denn die Einbindung weiterer Handlungsalternativen ermöglicht den Erwerb zusätzlicher Perspektiven, die wiederum Gegenstand von Analyse und Reflexion sind und ihrerseits einen neuen Reflexionszyklus ermöglichen. Hierfür sind weiterführende Reflexionsfragen vorgesehen, die auf eine kontinuierliche Fortsetzung des MuViT-Unterrichtskonzeptes abzielen:

- Was plane ich für die nächsten Monate/für das nächste Schuljahr in Bezug auf die Förderung der Mehrsprachigkeit im eigenen Unterricht?

- Welche Unterrichtsresultate kann ich/können wir (als Kollegium) nachweisen und anderen zur Verfügung stellen?

Unterstützt wird das reflexive Lernen im Basismodul durch ein Begleitportfolio. Es dient in dem MuViT Teacher Education Curriculum zur Dokumentation der dargestellten Lernphasen.

\subsection{Modul 1: Theoretische Grundlagen}

In diesem Teilbereich werden die theoretischen Grundlagen, auf denen das Modell einer Mehrsprachigkeitsdidaktik nach MuViT aufbaut, vorgestellt und gemeinsam mit den Lehrkräften erarbeitet. Dazu gehören die thematischen Schwerpunkte: Mehrsprachigkeit, Language Awareness und Multiliteralität. Für die Lehrkräfte wird somit ein theoretisches Gerüst geschaffen, in das sie ihre bisherigen Erfahrungen und ihr Wissen integrieren können und mit dem sie befähigt werden, das neu Erlernte in pädagogisch-didaktische Handlungen umzusetzen. 
Ziele des Moduls sind:

- Sensibilisierung für Mehrsprachigkeit, Heterogenität sowie Förderung der Interkulturalität im eigenen Unterricht (Baustein 1).

- Erweiterung der Kenntnisse zu Language Awareness, Aufbau theoretischen Wissens sowie die Aneignung von Fachtermini (Baustein 2).

- Kennenlernen von Grundlagen der Multiliteralitätspädagogik und verschiedener Dimensionen von Literalität sowie Aspekten des digitalen Lernens (Baustein 3).

Im Einklang mit dem beschriebenen reflexiven Ansatz erfolgt in Modul 3 schließlich eine Auseinandersetzung mit den theoretischen Grundlagen. Hier findet gleichfalls eine alternierende Reflexion in Bezug auf die eigenen Kenntnisse und Erfahrungen statt, die wiederum mit dem neu erworbenen Wissen abgeglichen werden. Im Zentrum steht dabei aus Sicht der Lehrkräfte stets die Frage, wie das neu Erlernte sinnvoll in die eigene Handlungspraxis umgesetzt werden kann.

Folgende Reflexionsfragen leiten diesen interaktiven Prozess von Aneignung und Transfer an:

- Was verstehe ich unter dem Begriff „Mehrsprachigkeit“"?

- Wie kann meiner Ansicht nach Mehrsprachigkeit gefördert werden?

- Welche theoretischen Hintergründe zu Language Awareness kenne ich?

- Welche Aspekte interessieren mich am meisten - und warum?

- Was verbinde ich mit dem Begriff „Multiliteralität“?

- Welche Ziele und Vorteile hat meiner Ansicht nach das digitale Lernen?

\subsection{Modul 2: Einführung in die MuViT Software}

Um die Talking Books (digitale, mehrsprachige Geschichten) ${ }^{1}$ und das Authoring Tool, mit dem selbst mehrsprachige Geschichten verfasst werden können, für den eigenen Unterricht zu nutzen, erfolgt in diesem Teilbereich zunächst eine Einführung in die Software. Die Lehrkräfte werden mit deren Funktionen und Nutzungsweisen vertraut gemacht. Sie haben zudem die Gelegenheit, eigene Lernerfahrungen mit der MuViT Software zu sammeln (Baustein 1 und 2). Im ersten Schritt sollen die Lehrer/-innen an den sicheren Umgang mit der Software herangeführt werden. Außerdem erfolgen eine vertie-

\footnotetext{
${ }^{1}$ Die Talking Books sind unter dem Titel My first stories im Oldenbourg Verlag, München erhältlich.
} 
fende Auseinandersetzung mit den Multiliteracy-Dimensionen (siehe MuViT Group 2012) und schließlich der Aufbau digitaler Kompetenzen. Wiederum bieten Reflexionsfragen einen Leitfaden für die individuelle Auseinandersetzung mit dem bereits Gelernten:

- Was haben die MuViT-Aufgaben gemeinsam?

- Was kann durch die MuViT bei meinen Schüler/-innen gefördert werden?

- Wie stehen Multiliteracy und Language Awareness zueinander?

- Welche Vorteile bringt meiner Meinung nach Multiliteracy im Hinblick auf Language Awareness?

- Welche Kompetenzen werden durch Multiliteracy gefördert?

\subsection{Modul 3: MuViT im Unterricht}

In diesem Modul werden angehende und bereits tätige Lehrkräfte angeleitet, Umsetzungen für den eigenen Unterricht $\mathrm{zu}$ entwickeln und diese in einer individuellen Praxisphase zu erproben. Folglich findet ein weiteres Mal eine enge Verzahnung von eigenen Erfahrungen und Kenntnissen und dem neu Erlernten statt. Durch die Erprobung im eigenen Unterricht wird die Relevanz der Inhalte für Lehrkräfte nachvollziehbar. Da der Transfer des Gelernten in die eigene Unterrichtspraxis besonders relevant für den Erfolg einer Qualifizierung ist, setzt sich das letzte Modul aus sechs Bausteinen zusammen, die wiederum bedarfsbezogen variiert werden können: In einem ersten Schritt erfahren Unterrichtende, wie sie die MuViT Software in ihren Sprachunterricht einführen können (Baustein 1). Hier sind verschiedene Varianten möglich, die innerhalb der Gruppe thematisiert werden. Anschließend erfolgt die Entwicklung einer Unterrichtseinheit (Baustein 2), die auf die eigene Lerngruppe zugeschnitten ist. Diese soll anschließend in einer Praxisphase (Baustein 5) in der eigenen Klasse oder einer Praktikumsklasse erprobt und später mit den anderen Fortbildungsteilnehmerinnen und -teilnehmern ausgewertet werden (Baustein 6). Da nicht immer die Möglichkeit der eigenen Erprobung besteht, können Lehrkräfte alternativ Ideen und Anregungen am Beispiel einer vorbereiteten Unterrichtseinheit (Baustein 3) ableiten. Die Einsatzmöglichkeiten des Authoring Tools (Baustein 4) stellen hier noch einmal ein gesondertes Fortbildungselement dar, da es ggf. zusätzlicher Inhalte zu schreibbezogenen Fähigkeiten bedarf, auf die dann entsprechend eingegangen wird.

Das letzte Fortbildungsmodul zielt vor allem darauf ab, dass Lehrkräfte Möglichkeiten kennen lernen, wie sie Schülerinnen und Schüler zum Lesen und Hören von Texten in verschiedenen Sprachen motivieren und zum sinnvollen und verantwortungsvollen Umgang mit dem Computer anregen können. Gleichzeitig sollen Lehrkräfte konkrete Handlungspraktiken, mit denen sie die Akti- 
vitäten zur Förderung von Mehrsprachigkeit in eine Unterrichtseinheit bzw. in ein ganzheitliches didaktisches Sprachlehrkonzept einbetten können, kennen lernen und erproben.

Während sich die Lehrkräfte in den vorangegangenen Modulen auf eine reflexive Weise mit den theoretischen und konzeptuellen Grundlagen auseinandergesetzt haben, werden sie nun mit der Analyse von Einsatzmöglichkeiten, denen die theoretischen Inhalte zu Grunde liegen, konfrontiert. Beispielsweise sollen sie in Kleingruppen einen Videoausschnitt, in dem Grundschüler/-innen mit der MuViT Software arbeiten, analysieren. Ihre Aufgabe ist es, herauszuarbeiten, wie die Schüler/-innen mit der Software bzw. mit den Language Awareness Aktivitäten umgehen und welche sprachlichen Aktivitäten dort stattfinden.

Für die analytische Arbeitsphase eignen sich folgende Reflexionsfragen:

- Was genau machen die Schüler/-innen? Wer hilft wem? In welchen Sprachen wird gesprochen? Was für ein Ziel hat diese Aufgabe für die Sprachentwicklung der Schüler/-innen?

- Welche Kompetenzen sollen die Schüler/-innen erlangen und wie tragen die Aufgaben dazu bei? In welcher Reihenfolge sind die Aufgaben konzipiert?

- Welche mehrsprachigen Elemente sind in dieser didaktischen Einheit vorhanden?

- Welche Rolle spielt meiner Ansicht nach die Sprachreflexion in Bezug auf die zu erreichenden Kompetenzen?

Die reflexive Aufbauphase kann ihrerseits durch folgende Reflexionsfragen unterstützt werden:

- Was habe ich Neues erfahren und gelernt?

- Was kann ich daraus für meinen Unterricht nutzen?

- Welche offenen Fragen sind noch zu klären?

Schließlich können folgende Reflexionsfragen den Erprobungs- und Evaluationsprozess begleiten:

- Wie habe ich MuViT in meinem Unterricht eingesetzt?

- Wie ging es mir als Lehrende/r in dem MuViT-Unterricht?

- Was konnte ich bei meinen Schüler/-innen beobachten?

- Würde ich MuViT wieder einsetzen? Wenn ja, wie?

Um das pädagogische und didaktische Handeln und Denken in der Unterrichtspraxis reflexiv zu verbessern und um Alternativen sichtbar zu 
machen, die den Lehrkräften in ihrem Lern- und Weiterbildungsprozess weiterhelfen können, wird die weitere Arbeit mit dem Portfolio empfohlen.

\section{$4 \quad$ Wirkung in der Lehrerbildung}

Das beschriebene Teacher Education Programm wurde innerhalb von Pilotkursen in Deutschland und Spanien erprobt. Dabei zeigte sich zum einen, dass durch das Modell eine tiefergehende Reflexion über Inhalte und Umsetzungsmöglichkeiten eines multilingualen Sprachunterrichts erreicht werden konnte. Zum anderen stellte sich heraus, dass die Lehrkräfte im Anschluss an das Modul 1 (Theoretische Grundlagen) in der Lage waren, Ideen für die Praxis innerhalb der Arbeit mit dem Sprachenportfolio zu integrieren. Es entstanden dadurch fundierte und begründete Praxisvorschläge für den eigenen Unterricht, deren Aktivitäten gemäß dem MuViT-Projekt auf der Grundlage von Language Awareness beruhen. Ein vorläufiges Ergebnis ist zudem, dass der Kompetenzanstieg im Hinblick eines an Mehrsprachigkeit orientieren Unterrichts dann bei Lehrkräften höher ausfällt, wenn die Fortbildung in professionsgemischten Teams (z. B. Deutsch-, Fremdsprachen- und Herkunftssprachenlehrkräfte) erfolgt. In welchen Teilbereichen (z. B. sprachliche Expertise) die verschiedenen Expertengruppen hierbei konkret voneinander profitieren, wird derzeit noch untersucht.

\section{Abschlussbemerkungen}

Das hier skizzierte MuViT Teacher Education Curriculum ermöglicht einerseits eine integrale Weiterbildung der Lehrkräfte, weil es eine kontinuierliche Wechselbeziehung zwischen dem Eigenen und Neuen darstellt. Es dient dazu, sich über das eigene mentale Sprachlehrkonzept bewusst zu werden und dieses ggf. zu modifizieren. Andererseits beinhaltet die auf das reflexive Erfahrungslernen ausgerichtete Gestaltung der Module eine unmittelbare Verzahnung der eingangs dargestellten drei Kompetenzdimensionen - Einstellungen, Kenntnisse und Fähigkeiten. Damit wird ein Scaffolding gewährleistet, bei dem die Lehrkräfte in ihrer Rolle als Lernende unterstützt werden. Sie erhalten neben theoretischem Input immer wieder Denkanstöße, die sie dazu an-regen sollen, alte Sichtweisen zu überdenken und das Gelernte in neue Denkstrukturen zu integrieren. Das vorliegende Teacher Education Modell dient damit primär zur Sensibilisierung von Lehrkräften für einen Sprachunterricht, der Mehrsprachigkeit zum Ausgangspunkt und als Bildungsziel hat. 


\section{Bibliographie}

Blömeke, Siegrid (2005). Lehrerausbildung - Lehrerhandeln - Schülerleistungen. Perspektiven nationaler und internationaler empirischer Bildungsforschung. Berlin: Forschungsabteilung der Humboldt-Universität zu Berlin. Online: http://edoc.hu-berlin.de/ humboldt-vl/139/bloemeke-sigrid 3/PDF/bloemeke.pdf (letzter Aufruf [23.03.2013]).

Cantone, Katja (2011). Mehrsprachigkeit und ihre Bedeutung in der Lehrerausbildung. In: Elsner, Daniela / Wildemann, Anja (Hrsg.): Sprachen lernen - Sprachen lehren. Perspektiven für die Lehrerbildung in Europa. Language Learning - Language Teaching. Prospects for Teacher Education across Europe. Frankfurt/M.: Lang. 23-35.

Elsner, Daniela (2010). Englisch lernen als dritte Sprache: Was unterscheidet den zwei- oder mehrsprachigen vom einsprachigen Fremdsprachenlerner? Praxis Fremdsprachenunterricht 2/2009: 4-8.

Esteve, Olga (2010). Desconstruir per tornar a construir: cap a una metodología per al tractament integrat de llengües a les escoles. In: Guasch, Oriol (Hrsg.): El Tractament Integrat de Llengües. Barcelona: Grao. 145-163.

Esteve, Olga / Melief, Ko / Alsina, Ángel (Hrsg.) (2010). Creando mi profesión. Una propuesta para el desarrollo profesional del profesorado. Barcelona: Editorial Octaedro.

Frederking, Volker/Jonas, Hartmut (Hrsg.) (2008). Neue Medien im Deutschunterricht eine Zwischenbilanz. Online: http://www.medid.de/home. (letzter Aufruf: [20.04.2013]).

Grossmann, Schimon (2008). Supplementing Textbooks with Computer-Based Resources in the Primary EFL-Classroom. (unpublished Master Thesis). Freiburg: Pädagogische Hochschule Freiburg.

Hansen, Georg (2010). Mehrsprachigkeit der Europäer - Programmatik und empirische Realität. In: Krüger-Potratz, Marianne / Neumann, Ursula/Reich, Hans H. (Hrsg.): Bei Vielfalt Chancengleichheit. Münster: Waxmann. 306-315.

Hawkins, Eric (1984). Awareness of Language. An Introduction. Cambridge.

Holzbrecher, Alfred (Hrsg.) (2011). Interkulturelle Schule. Eine Entwicklungsaufgabe. Schwalbach: Wochenschau Verlag.

NLG - New London Group (1996). A Pedagogy of Multiliteracies: Designing social Futures. Harvard Educational Review 66/1. 1-30. Online: http://wwwstatic.kern.org/filer/blog Write44ManilaWebsite/paul/articles/A_Pedagogy_of_Multiliteracies_Designing_Social_F utures.htm (letzter Aufruf [15.02.2013]).

Katsarou, Eleni (2009). A Multiliteracy Intervention in a Contemporary „Mono-Literacy“ School in Greece. The International Journal of Learning 16/5: 55-65.

KIM-Studie (Medienpädagogischer Forschungsverband Südwest) (2006). Kinder + Medien, Computer + Internet. Basisuntersuchung zum Medienumgang 6- bis 13-Jähriger. Online: http://www.mpfs.de/fileadmin/KIM-pdf06/KIM2006.pdf (letzter Aufruf [22.03.2013]).

KIM-Studie (Medienpädagogischer Forschungsverband Südwest) (2010). Kinder + Medien, Computer + Internet. Basisuntersuchung zum Medienumgang 6- bis 13-Jähriger. Online: http://www.mpfs.de/fileadmin/KIM-pdf10/KIM2010.pdf (letzter Aufruf [20.03.2013]).

Korthagen, Fred (2001). Linking Practice and Theory. The Pedagogy of Realistic Teacher Education. London: Lawrence Erlbaum Associates. 
Krumm, Hans-Jürgen (2010). Erziehungsziel Mehrsprachigkeit. In: Krüger-Potratz, Marianne / Neumann, Ursula / Reich, Hans H. (Hrsg.): Bei Vielfalt Chancengleichheit. Münster: Waxmann. 289-295.

Lankes, Eva-Maria (Hrsg.) (2008). Pädagogische Professionalität als Gegenstand empirischer Forschung. Münster: Waxmann.

Lipowsky, Frank (2011). Theoretische Perspektiven und empirische Befunde zur Wirksamkeit von Lehrerfort- und -weiterbildung. In: Terhart, Ewald / Bennewitz, Hedda / Rothland, Martin (Hrsg.): Handbuch der Forschung zum Lehrerberuf. Münster: Waxmann. 398-417.

Luchtenberg, Sigrid (2010). Language Awareness. In: Oomen-Welke, Ingelore /Ahrenholz, Bernt (Hrsg.): Deutsch als Zweitsprache. 2. Aufl. Schneider: Baltmannsweiler. 107-117.

MuViT-Group (2011). MuViT - Multilingual Virtual Talking Books. Project Flyer. Brussels: European Commission. Online: www.mu-vit.eu (letzter Aufruf [16.05.2013]).

MuViT-Group (2012). Grundlegende Informationen für die Lehrerfortbildung. Online: www.mu-vit.eu/Teacher Materials/Login: teacherMuViT (erhältlich in den Sprachen Deutsch, Englisch, Spanisch, Russisch, Türkisch) (letzter Aufruf [16.05.2013]).

Neuner, Gerhard (2003). Mehrsprachigkeitskonzept und Tertiärsprachendidaktik. In: Hufeisen, Britta/Neuner, Gerhard (Hrsg.): Mehrsprachigkeitskonzept - Tertiärsprachenlernen Deutsch nach Englisch. Straßburg: Europäisches Fremdsprachenzentrum. 13-34.

Oomen-Welke, Ingelore (1999). Sprachen in der Klasse. Praxis Deutsch 09/1999: 14-23.

Oomen-Welke, Ingelore (2010). Didaktik der Sprachenvielfalt. In: Oomen-Welke, Ingelore / Ahrenholz, Bernt (Hrsg.): Deutsch als Zweitsprache. 2. überarb. Aufl. Schneider: Baltmannsweiler. 479-492.

Pünter, Mathias (2012). Mehrsprachigkeit im Deutschunterricht aus der Sicht von Lehrkräften. Einstellungen, Erfahrungen und Wünsche. Eine empirische Untersuchung an einer niedersächsischen Grundschule. In: Elsner, Daniela / Wildemann, Anja (Hrsg.): Mehrsprachigkeit und Unterrichtsforschung. In der Reihe Papers of Excellence. Ausgewählte Arbeiten aus den Fachdidaktiken. Aachen: Shaker. 57-91.

Rank, Astrid (2012). Wie sind Studentinnen des Grundschullehramts auf sprachliche und kulturelle Heterogenität vorbereitet? Zeitschrift für Grundschulforschung 5/2: 79-93.

Schumann, Stephan / Eberle, Franz (2009). Überlegungen zur Erfassung von langfristigen Effekten der Professionalität von Lehrenden. In: Zlatkin-Troitschanskaia, Olga / Beck, Klaus/ Sembill, Detlef / Nickolaus, Reinhold / Mulder, Regina (Hrsg.): Lehrerprofessionalität. Bedingungen, Genese, Wirkungen und ihre Messung. Weinheim und Basel: Beltz. 718727.

Weinert, Franz E. (2001). Leistungsmessungen in Schulen. 2. Aufl. Weinheim und Basel: Beltz.

Wildemann, Anja (2010). „Eigentlich spreche ich nur Kurdisch und Deutsch“. Sprachinteresse und Sprachenselbstbewusstsein mehrsprachiger Schülerinnen und Schüler. In: Merklinger, Daniela / Jantzen, Christoph (Hrsg.): Lesen und Schreiben: Lernerperspektiven und Könnenserfahrungen. Freiburg i. B.: Fillibach. 215-232.

Wildemann, Anja (2011). Multiliteralität als Ausgangspunkt und Zielperspektive auf dem Weg in die Schrift. In: Hüttis-Graff, Petra / Wieler, Petra (Hrsg.): Übergänge zwischen 
Mündlichkeit und Schriftlichkeit im Vor- und Grundschulalter. Freiburg i. B.: Fillibach. 273-290.

Wildemann, Anja (i. Dr.). Multiliterale Kompetenzen fördern. In: Dirim, Inci / Oomen-Welke, Ingelore (Hrsg.): Deutschunterricht im mehrsprachigen Klassenzimmer. Stuttgart: Fillibach bei Klett. 


\title{
Zur Ausbildung und Entwicklung adaptiver Lehrkompetenz von angehenden Fremdsprachen- lehrerinnen und -lehrern durch Videofeedback
}

\section{Claudia Frevel (Siegen)}

\begin{abstract}
The ability of self-reflection plays a central role in the development and construction of a professional identity. This paper proposes a concept for the apprenticeship of preservice teachers, which focuses especially on the initiation of self-reflection by means of video-feedback. The experience of a student teacher serves as an example of how the conscious and reflective teaching approach can lead to an increase in teaching skills and therefore to the formation of a professional identity.

Para el desarrollo y la formación de una identidad profesional la capacidad de reflexión figura un papel importante. El siguiente artículo tematiza un concepto para la formación de profesores. Este concepto inicia procesos de reflexión a base de feedback por video. El ejemplo de una profesora en práctica muestra hasta que punto el trato consciente y reflexivo de acciones del profesor en clase le facilita a una competencia adaptiva y desarrolla por lo tanto su identidad profesional.
\end{abstract}

\section{$1 \quad$ Einleitung}

Die Fähigkeit zur Selbstreflexion spielt eine zentrale Rolle bei der Entwicklung und Ausbildung einer beruflichen Identität. Diese setzt sich in Anlehnung an Krewer/Eckensberger (1998) zusammen aus: dem (kognitiven) beruflichen Selbstkonzept, dem (affektiven) beruflich-professionellen Selbstwert und dem (konativen bzw. handlungsleitenden) beruflichen Selbstvertrauen. Da der Identitätsentwicklung immer ein dialogisch-kommunikativer Austausch zwischen der eigenen Perspektive und den durch die soziale Umwelt widergespiegelten Perspektiven zugrunde liegt (vgl. Mead 2005), kann ein derartiger Kommunikationsprozess in der Lehrerausbildung gezielt gefördert und auf beruflich relevante Aspekte des Selbstkonzeptes bezogen werden. Selbstreflexive Prozesse, die berufliche Selbstkonzepte stärker ins Bewusstsein rücken, beeinflussen die Ausbildung berufsspezifischer Einstellungen. Sie lenken die Bewertung des eigenen Lehrerhandelns, sie bestimmen die Qualität der eigenen Unterrichtspraxis etc. Reflexion im Lehrerberuf wird insofern als die Schlüsselbedingung zur nachhaltigen Unterrichtsentwicklung erachtet (Helmke 2009: 118), als dass sie zur Entwicklung eines professionellen Lehrer-Selbstkonzeptes beitragen kann. In der Diskussion um die Reform der Fremdsprachenlehrerausbildung herrscht inzwischen Konsens über die Notwendigkeit, dass selbstreflexive Prozesse während der Ausbildung noch stärker und gezielter zu initiieren sind, da z. B. eigene Sprachlernerfahrungen sich unmittelbar auf die Vorstellung über Aufgaben und Funktionen des Fremdsprachenlehrers auswirken 
(vgl. Caspari 2003). Es macht insofern Sinn, Erfahrungswissen und theoretische fachdidaktische und fachwissenschaftliche Erkenntnisse während des Lehramtsstudiums systematisch zu verknüpfen (vgl. Schocker-v. Ditfurth 2001).

In meinem Beitrag werde ich nachfolgend ein Konzept für die zweite Ausbildungsphase des Fremdsprachenlehrers im Fach Spanisch (Referendariat) vorstellen, das explizit darauf abzielt, angehende Fremdsprachenlehrer/innen in eine intensive Auseinandersetzung mit der eigenen Unterrichtspraxis zu führen und in diesem Zusammenhang ihr berufliches Selbstkonzept zu reflektieren. Dazu werden auf der Grundlage von Unterrichtsnachbesprechungen und Videofeedback Reflexionsprozesse initiiert, die zum Ziel haben, die Lehrperson zu einem kritischen und distanzierten Nachdenken über das eigene unterrichtspraktische Handeln zu führen und über die Einstellungen, die diesem möglicherweise zugrunde liegen. Anhand von Gesprächsmitschnitten wird untersucht, inwieweit ein bewusster und reflektierter Umgang mit den im Unterricht praktizierten und beobachteten Handlungen einen sukzessiven Kompetenzaufbau im Sinne einer ,adaptiven Expertise“ (siehe Kap. 3 weiter unten; vgl. auch Herzog 2011: 68) ermöglicht.

\section{Das Ausbildungskonzept}

Gegenstand des vorliegenden Beitrags sind berufsbegleitende Reflexionsgespräche, die Referendarinnen und Referendare des Faches Spanisch über den eigenen Unterricht führen. Diese Gespräche finden in Form von Unterrichtsnachbesprechungen (NB) statt, die regulär im Anschluss an eine durchgeführte Unterrichtsstunde zwischen Seminar- und/oder Fachleiter/in, die den Unterricht eingesehen haben, und der Referendarin/dem Referendar durchgeführt werden. Ziel dieser Besprechungen ist es unter anderem, die Lehrperson zur kritischen (Selbst-)Reflexion über das eigene unterrichtspraktische Handeln anzuleiten und anzuregen.

Das Fachseminar Spanisch am Zentrum für schulpraktische Lehrerausbildung (ZfsL) in Siegen stellt im Rahmen der fachdidaktischen und fachpraktischen Ausbildung ein erweitertes Konzept bereit, das eine intensive Auseinandersetzung mit dem durchgeführten Unterricht auf der Grundlage eines Videomitschnitts vorsieht. Dazu wird der eingesehene Unterricht auf Video aufgenommen und der Lehrperson im Anschluss an den Unterrichtsbesuch mit dem Auftrag ausgehändigt, das Unterrichtsvideo zwei Mal in Ruhe anzuschauen und drei bis vier Szenen oder Situationen zu markieren. Über diese führen dann die Fachleiterin und die Referendarin/der Referendar ein zweites Reflexionsgespräch. Die Arbeit mit Video beruht auf Freiwilligkeit und dient in erster Linie dazu, weiterführende Reflexionen der Lehrperson $\mathrm{zu}$ initiieren und $\mathrm{ihr} \mathrm{zu}$ 
ermöglichen, die eigene Unterrichtspraxis aus einer Außenperspektive zu erfahren.

Der eingesehene Unterricht wird insofern zwei Mal aus unterschiedlichen Retrospektiven besprochen: Während die erste Nachbesprechung (NB I) zu der Unterrichtsstunde in Form eines strukturierten Feedback- und Beratungsgesprächs erfolgt, bei dem Referendar/in und Fachleiter/in das erlebte und beobachtete Unterrichtsgeschehen vor dem Hintergrund des unmittelbar Erfahrenen rekonstruieren, analysieren und reflektieren, findet die zweite Nachbesprechung (NB II) zu der gleichen Stunde anhand des Unterrichtsvideos und mit einer zeitlichen Distanz statt.

\section{Die Daten}

Im Rahmen einer Studie, die die Universität Siegen in Kooperation mit dem ZfsL-Siegen durchführt, wird zurzeit dieses Ausbildungskonzept evaluiert. Dazu wurden in einem Zeitraum von vier Jahren (Februar 2009 bis Februar 2013) die in diesen Nachbesprechungen geführten Gespräche (NB I und NB II) von zehn Referendarinnen/Referendaren zu drei unterschiedlichen Ausbildungszeitpunkten aufgenommen, und zwar zu Beginn (1. UB), zur Hälfte (2./3. UB) und zum Ende des Ausbildungszeitraums (4./5. UB) (vgl. Tabelle 1).

Tabelle 1: Aufnahmezeitpunkte

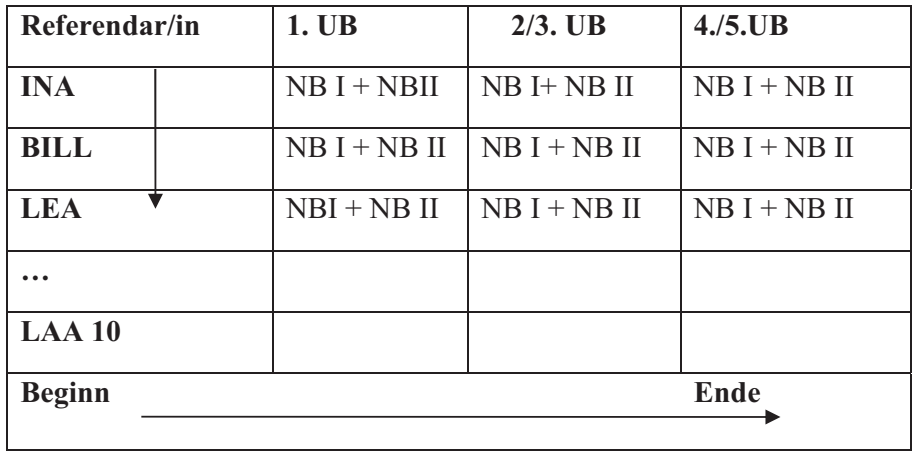


Diese Gespräche werden transkribiert und inhaltsanalytisch ausgewertet. Ziel ist es, in einer Langzeitstudie aufzuzeigen, inwieweit sich Überzeugungen und Vorstellungen über die eigene Berufspraxis und somit das berufliche Selbstkonzept verändern und wie sich in diesem Ausbildungskontext Handlungskompetenzen nachhaltig (aus-) und weiterbilden (vgl. Abendroth-Timmer 2011; AbendrothTimmer/Frevel 2013). Bevor ich nachfolgend aus der Studie ein Fallbeispiel vorstelle, werde ich im folgenden Kapitel zunächst den theoretisch-methodischen Rahmen skizzieren.

\section{Theoretisch-methodischer Rahmen}

\subsection{Erfahrungswissen und Reflexion}

Die Ausbildung von Lehrerprofessionalität ist an die Entfaltung einer Erfahrungsbasis gebunden, die sich durch die reflexive Verarbeitung unterschiedlicher Kontexte und Wissensbestände entwickelt und in die Entwicklung eines differenzierten beruflichen Selbstkonzeptes mündet. Auf die Frage, welches Wissen und welche Kompetenzen Lehrpersonen zur Ausbildung von Professionalität benötigen, betont Walter Herzog (2011), dass es offensichtlich diagnostisches (analytisches) Wissen ist, das Berufspraktiker bzw. Lehrpersonen brauchen, weniger aber technisches (synthetisches) Wissen: „Die Situation des Unterrichts ist $\mathrm{zu}$ komplex, als dass Lehrerinnen und Lehrer aus wissenschaftlichen Erkenntnissen direkten Nutzen für die Anleitung zum Handeln ziehen könnten" (ebd.: 63). Wissenschaftliches Wissen als Grundlage für die berufliche Praxis sei daher unzureichend, Lehrpersonen benötigten zusätzliche Wissensbestände, um erfolgreich handeln zu können (ebd.: 64). Dieses zusätzliche Wissen lasse sich als Erfahrungswissen bündeln, das sich aus Alltagswissen (Erfahrungen im Umgang mit Menschen), Beobachtungswissen (während der eigenen Schulzeit erworben) und Berufswissen (alltägliche Erfahrungen in der Lehrertätigkeit) zusammensetzt. Herzog (ebd.: 68) betont, dass dieses Erfahrungswissen zwingend einer reflexiven Kompetenz bedürfe, die von wissenschaftlichen Wissensformen angeleitet werden müsse.

Wichtig an diesem Ansatz ist die Einsicht, dass Erfahrungswissen und wissenschaftliches Wissen unterschiedlich strukturiert sind: Erfahrungswissen orientiert sich nicht an Logik, sondern am Beispielhaften ${ }^{1}$. Es ist nicht diszipli-

1 Siehe auch Beck et al. (2008): Die Autoren betonen in Anlehnung an die intuitiven Verhaltenstheorien nach Groeben, Wahl et al. (1988) und Heider (1958), dass ,diese Theorien zeigen, dass das pädagogische, das diagnostische und das didaktische Wissen von Lehrpersonen nicht lexikalisch oder lehrbuchartig gespeichert ist, sondern in situationsspezifischer handlungsorientierter Form auf Grund der durch das Unterrichten als Lehrperson gemachten Erfahrung“ (ebd.: 23). 
när, sondern situations- und fallspezifisch organisiert. So sind es die erlebten Situationen und vergleichbaren Fälle, an die sich der Praktiker erinnert und die er in sein Erfahrungsnetz einbindet (vgl. Herzog 2003). Experten agieren auf der Basis von Erinnerungen an ähnliche Situationen (vgl. Schön 1983: 138), sie richten ihr Handeln an typischen Situationen und exemplarischen Handelsläufen aus (vgl. Berliner 1992; Bromme 1992: 149). Um der Wirklichkeit bzw. der Komplexität des beruflichen Alltags eine Ordnung zu geben, nutzt der Praktiker die Kraft der Analogie und der Metapher (Herzog 2011: 64 ff.). Es reicht somit nicht, der Lehrperson theoretisches Wissen über das Unterrichten anzubieten, sondern es geht vielmehr darum, eine Investigationshaltung zu vermitteln, die ihr hilft, den eigenen „Standpunkt zu dezentralisieren, neue Sichtweisen zu generieren und der Wirklichkeit alternative Deutungen abzugewinnen" (ebd.: 69) $)^{2}$. Im Referendariat muss Erfahrungswissen insgesamt Teil des beruflichen Selbstkonzeptes werden. Videostimulierte Reflexionsgespräche können einen Beitrag dazu leisten, das neue Erfahrungswissen auf das bisherige Selbstkonzept zu beziehen und dieses zu rekonstruieren.

Damit erweist sich das Paradigma von der Anwendung des Theoriewissens in der Praxis als problematisch, denn die Annahme, dass zwischen Wissen und Handeln eine deduktive Beziehung bestehe, kommt eher einer technologischen Interpretation von Theorie und Praxis gleich (Messner/Reusser 2000).

Darauf hebt auch Schüpbach (2007) ab, wenn er betont, dass das Wissen sich im Handeln manifestiere, ,es erwächst aus dem Handeln, aus der Praxis und kann allenfalls nachträglich in der reflexiven Auseinandersetzung mit den Erfahrungen explizit formuliert werden“ (ebd.: 50) ${ }^{3}$. Professionelles Wissen kompetenter Lehrpersonen spiegelt sich zugleich in ihren Entscheidungen und Urteilen, in ihren Überlegungen und Begründungen des unterrichtlichen Handelns, in ihren Fragestellungen und Wahrnehmungen zum Unterrichtsverlauf. Hier manifestiert sich praktisch gewordenes Handlungswissen (vgl. Messner/Reusser 2000: 282).

Wissenschaftliche Forschungsergebnisse aus der Lehrerbildung finden sich diesem Ansatz folgend als Reflexionsgrundlage in der Ausbildung wieder, nicht aber als Anleitung. Interessant ist, dass dem wissenschaftlichen Wissen damit eine konkrete spezifische Funktion zukommt, indem es als „Grundlagenwissen [...] für den Praktiker Hintergrundwissen darstellen [kann], mit dem er Probleme in seinem Arbeitsfeld besser durchschauen und daraufhin Lösungen

\footnotetext{
${ }^{2}$ Siehe hierzu auch das Konzept der Aktionsforschung (Altrichter/Posch 1998).

${ }^{3}$ Mit diesem Ansatz, dem „Wissen in/aus der Praxis“ ist das Konzept der subjektiven Theorien eng verbunden, bei dem der handelnde und seine Handlungen reflektierende Mensch im Zentrum steht.
} 
suchen kann." (Dann 1994: 175, zitiert nach Beck et al. 2008: 53). Für den Reflexionsprozess bedeutet dies insofern, dass es ,abgestützte und verlässliche ,Anstöße von außen'“ (Schüpbach 2007: 55) braucht, damit sich Lehrerinnen und Lehrer bei der Reflexion über ihr berufliches Handeln nicht im Kreise drehen. „Das Theoriewissen ist deshalb für den Praktiker, der sein Lehrerwissen entwickeln und professionalisieren will, unverzichtbar" (Schüpbach 2007: 55). Um in diesem Sinne professionelle Handlungskompetenz zu entfalten, ist es wichtig, dass die Reflexion neue Handlungsoptionen eröffnet, um das eigene Handlungsrepertoire zu überprüfen und zu erweitern.

Professionalität ist vor dem Hintergrund dieses Ansatzes somit ein Individualmerkmal und eine qualitative Eigenschaft der einzelnen Lehrkraft (vgl. Herzog 2011: 66), die professionell handelt, „wenn sie fähig ist, die vielschichtige Wissensbasis ihres Lehrerberufs flexibel zu nutzen, situativ zu verdichten und durch Reflexion ihre Erfahrungen kontinuierlich zu erweitern" (Herzog 2011: $68)$.

\subsection{Situiertes Lernen und adaptive Lehrkompetenz}

Für die Lehrerbildung bedeutet dies, dass es gilt, Lernsituationen zu schaffen, in denen zukünftige Lehrerinnen und Lehrer die Gelegenheit erhalten, die verschiedenen Wissensformen und ihr vor diesem Hintergrund entwickeltes Selbstkonzept, zu reflektieren und zu vernetzen, da dieses ihr Handeln begründet. Dies findet z.B. im „Situierten Lernen“ (vgl. Beck et al. 2008) statt.

Das Konzept des Situierten Lernens beruht auf dem Paradigma der Situated Cognition ${ }^{4}$ und besagt, „dass beruflicher Wissenserwerb nur dann erfolgreich ist, wenn er problemorientiert und in möglichst authentischen Situationen erfolgt" (Schüpbach 2007: 57). Ausgangspunkt ist, dass Wissen immer als Ergebnis einer individuellen Konstruktion und Lernen als ein aktiver konstruktiver Prozess in einem spezifischen Handlungskontext und im Rahmen sozialer Transaktionen zu sehen ist (Beck et al. 2008: 55).

Daher lässt sich der Prozess des Erwerbs professioneller Kompetenz eher als Lernen denn als Entwicklung beschreiben (vgl. Herzog 2011: 68), da der Entwicklungsbegriff eine „zu homogene Vorstellung vom Prozess der Lehrerwerdung" (ebd.: 68) suggeriert. Herzog (ebd.) plädiert daher dafür, professionelle Kompetenz als adaptive Expertise zu beschreiben.

\footnotetext{
${ }^{4}$ Weiterführende Literatur zur „Situated Cognition“ findet sich in Beck et al. (2008), siehe auch Schüpbach (2007)).
} 
Adaptiv ist die Kompetenz von Lehrerinnen und Lehrern dann, wenn sie fähig sind, nicht nur aus ihren beruflichen Erfahrungen zu lernen, sondern ihre Kompetenzen durch Lernen auch zu erweitern.

Erst die auf „Reflexion beruhende Integration jener Wissensformen, die wir als Basis von Lehrerprofessionalität erkannt haben: Alltagswissen, Beobachtungswissen, Berufswissen und wissenschaftliches Wissen" (ebd.: 68) führen zur Ausbildung professioneller Kompetenz im Sinne einer adaptiven Expertise.

Das oben beschriebene Ausbildungskonzept am Studienseminar Siegen entspricht diesen Prinzipien des situativen Lernens. Ziel ist es, die Referendarinnen und Referendare zu einer adaptiven Lehrkompetenz, wie sie Schön (1983) in seinem ,reflective practitioner" charakterisiert, zu befähigen.

Nach Beck et al. (2008) ist eine adaptive Lehrperson in der Lage,

Unterschiede bei den Lernenden und Schlüsselmomente in Lehr-Lern-Prozessen wie Nicht-Verstehen, Abschweifen oder Störungen sensibel wahrzunehmen und mit angemessenen didaktischen Maßnahmen darauf zu reagieren. (...) ,Adaptiv-sein' bedeutet aber auch, Situationsmomente und Handlungsalternativen im Lehr-Lerngeschehen - sowohl in der Unterrichtsplanung als auch während des Unterrichts - zu antizipieren und bereit zu sein zu reagieren, wenn eine Handlungsanpassung an eine neue Situation erwünscht bzw. erforderlich ist (ebd.: 39).

Inwieweit berufliches Lernen in dieser Form im Ausbildungszeitraum stattfindet, bzw. inwieweit es den Referendarinnen und Referendaren gelingt, Professionalität im Sinne einer adaptiven Expertise aufzubauen, wird zu untersuchen sein.

Im Rahmen dieses Beitrags geht es in erster Linie darum zu erfassen, wie die Lehrpersonen die Qualität und Effizienz ihres unterrichtlichen Handelns und ihrer Selbstwirksamkeit in den Nachbesprechungen beschreiben und beurteilen. Es wird gefragt, auf welches Wissen in den Nachbesprechungen jeweils zugegriffen wird und wie sich wahrgenommene und reflektierte Handlungskompetenzen im Ausbildungszeitraum im Sinne einer adaptiven Expertise entwickeln und entfalten. Nachzeichnen lässt sich dieser Lernprozess anhand der Gesprächsaufnahmen, die von den Nachbesprechungen (I und II) zu den verschiedenen Ausbildungszeitpunkten (1. UB bis 5. UB) erstellt wurden.

Im folgenden Beispiel werde ich Ina vorstellen. Von Ina liegen sowohl schriftliche Reflexionstexte als auch Gesprächsaufnahmen vor. $\mathrm{Zu}$ den schriftlichen Reflexionstexten zählen ein Selbstporträt sowie die im Anschluss an die Nachbesprechungen erstellten Zusammenfassungen, in welchen sie die in den Gesprächen jeweils thematisierten Beratungsaspekte sowie mögliche Handlungsalternativen niederschrieb. Diese Texte liegen in Form eines ausbildungsbegleitenden Portfolios (PORT) vor. Zugleich wurden die Nachbesprechungen (I und II) mit Hilfe eines Audiogerätes aufgenommen und transkribiert. 
Ausgewertet und analysiert werden die Reflexionstexte und -gespräche mit Hilfe des Programms MAXQDA.

\section{INA}

\subsection{Das berufliche Selbstkonzept}

Inas Lehrerpersönlichkeit wird geprägt durch einen hohen Anspruch an sich selbst. In den zwei Jahren des Referendariats nimmt sie die Möglichkeit der Videoreflexion gezielt wahr, da sie, wie sie sagt, durch das Videofeedback über sich selbst am meisten lerne:

weil ich mich dann halt da noch mal sehe. Weil ich mich von der Außenperspektive wahrnehme (...). Aber am meisten würde ich sagen, lerne ich da über mich selbst. [INA/2.UB/NB II: 147-153]

Intensiv setzt sie sich im Rahmen der Ausbildung mit sich und ihrer Lehrerpersönlichkeit auseinander. Sensibel und selbstkritisch reflektiert sie ihr Verhalten und ihre unterrichtlichen Handlungen, wenn es darum geht, Schülerinnen und Schüler z. B. in das Unterrichtsgeschehen einzubinden oder zur fremdsprachlichen Kommunikation anzuregen und zu aktivieren.

Getragen wird Inas Anspruch durch ein positives Schul- und Lehrerbild, das sich in ihrer Schulzeit als Schülerin bildete. In ihrem Portfolio [PORT] betont sie:

Insgesamt hat mir meine eigene Schule ein hohes Maß an Identifikationsmöglichkeiten eröffnet, so dass ich mich bis heute tief verbunden fühle sowohl mit der Schule im Allgemeinen als auch mit meinen ehemaligen - sehr engagierten - Lehrern. Diese positive Erfahrung, dass Schule nicht nur ein Ort des Lernens ist, sondern vielmehr ein ganz zentraler „Lebensraum“, zusammen mit dem Wunsch, mit jungen Menschen arbeiten zu wollen, sind die beiden wohl wichtigsten Faktoren für meine Entscheidung, den Lehrerberuf zu wählen. [INA/PORT: 4]

Diese Erfahrung, die Ina in und mit Schule machte, entspricht in hohem Maße ihrem Engagement und ihrer Bereitschaft, den Ausbildungsprozess aktiv (mit-) $\mathrm{zu}$ gestalten und kommt in ihrem Selbstkonzept deutlich zum Tragen: Kompetent, aber nicht allwissend, authentisch, offen, konsequent und fair im Umgang mit den Schülerinnen und Schülern, so beschreibt sie ihre Vorstellung davon, wie sie als Lehrerin wirken möchte:

Ich möchte als Spanischlehrerin vor allem kompetent wirken auf die Schülerinnen und Schüler. Das bedeutet aber nicht, dass ich auch gleichzeitig ,allwissend“ erscheinen möchte. Dass ich beispielsweise nicht jede Vokabel wissen kann, versteht sich von selbst, da ich keine Muttersprachlerin bin. Ich denke, dass die SuS es als weitaus sympathischer empfinden, wenn man „Schwächen“, sofern man dies so bezeichnen möchte, offen zugibt. Das Wichtigste, so glaube ich, ist es, authentisch zu bleiben. (...) Insge- 
samt möchte ich, dass die SuS etwas bei mir lernen und zwar mit Freude und Spaß. Die $\mathrm{SuS}$ sollen merken, dass ich konsequent und fair bin. [INA/PORT: 16]

Dabei hat Ina stets das Ziel des Fremdsprachenerwerbs im Blick, und zwar die Lernenden zu kompetenten Sprecherinnen und Sprechern auszubilden, die sich im Zielland angemessen verständigen können. Wortschatzarbeit und viel Kommunikation im Unterricht bilden daher für sie eine feste Grundlage für den schulischen Fremdsprachenunterricht (vgl. INA/PORT: 21).

Für diesen schulischen Fremdsprachenunterricht ist meines Erachtens die Wortschatzarbeit von besonderer Bedeutung. Zwar sollten grundlegende grammatische Kenntnisse vermittelt beziehungsweise vorhanden sein, aber diese sind für die Kommunikation im Ausland nicht so bedeutsam wie das Vokabular. Wenn man grammatikalische Fehler macht, wird man von den Einheimischen trotzdem verstanden, wohingegen fehlendes Vokabular weitaus schwerwiegendere Verständigungsprobleme verursacht. Ich glaube, dass die SuS von Anfang an zum Sprechen angeleitet werden sollten, um ihnen so die Hemmungen zu nehmen und ihnen bei einem Auslandsaufenthalt Erfolgserlebnisse zu ermöglichen. Wenn sie merken, dass sie sich im Zielland verständigen können, werden sie positiv bestärkt. Außerdem sollte die Zeit im ersten Lernjahr insbesondere in der Oberstufe dafür genutzt werden, den SuS kommunikative Erfahrungen zu ermöglichen, da sie im zweiten und dritten Lernjahr in Anbetracht der Vorbereitung auf das Abitur sehr viel (schriftliche) Textarbeit leisten müssen. [INA/PORT: 21]

Das hier skizzierte berufliche Selbstkonzept Inas findet sich in ihren Reflexionen (den Nachbesprechungen (I) und (II)) auf unterschiedlichen Ebenen wieder. So fokussiert sie in den Gesprächen häufig Unterrichtssituationen, in denen sie eine Diskrepanz spürt in Bezug auf ihren Anspruch, einen schülerorientierten Unterricht mit vielen Sprechanlässen zu organisieren, und ihrer Wirksamkeit, z.B. jeweilige Unterrichtsphasen durch eine angemessene Gesprächsführung oder Intervention durchzuführen. In ihren Reflexionen wird deutlich, wie wichtig es ihr ist, die Lernenden in alle Phasen des Unterrichts einzubinden, sie zu fremdsprachlicher Kommunikation anzuregen und $\mathrm{zu}$ aktivieren. Vor allem in den Videoreflexionen nimmt sie diesbezüglich die Schülerreaktionen sehr sensibel wahr. Anhand der Gespräche lässt sich vom 1. bis 5 . UB nachverfolgen, wie gezielt sie ihren eigenen Lernprozess steuert, um die Wirksamkeit ihrer unterrichtlichen Handlungen zu steigern. Dabei wird deutlich, wie sie zunehmend eine adaptive Lehrkompetenz aufbaut.

Wie sie die Wirksamkeit ihrer Intervention und Gesprächsführung im Nachhinein einschätzt und reflektiert, werde ich nachfolgend an einem Beispiel skizieren. 


\subsection{Reflexionen zur Selbstwirksamkeit}

\subsubsection{Die Textarbeit}

Im Rahmen ihres ersten UBs beschäftigt sich Ina in ihrer Reflexion vor allem mit der Wirksamkeit ihrer Interventionen, die sie im Nachhinein als wenig effektiv registriert. Als sie im Anschluss an den Unterricht von der Fachleiterin aufgefordert wird, die gerade durchgeführte Stunde zu kommentieren, gibt sie folgendes Statement ab:

INA: ich habe mich ein bisschen geärgert, dass ich da so ein bisschen durch gehetzt bin, ich denke, es hätten auch zwei Gruppen gereicht, um die heute vorzustellen, dann hätte ich eventuell z. B. den exemplarischen Text vielleicht doch noch mal lesen sollen, denn im Nachhinein kam halt heraus ,puesto de trabajo“ und so, das war dann doch nicht klar, da hätte ich genauer nachfragen müssen, nicht: „habt ihr das verstanden?“ oder „gibt es Fragen?“ und, ja, das halt hab ich direkt gemerkt. [INA/1.UB/NBI: 17]

In ihrer Kommentierung der Stunde spricht Ina drei Themenbereiche an: Sie sei „gehetzt“, (Thema: Umgang mit der zur Verfügung stehenden Zeit), sie erwähnt die Präsentationsphase, da „hätten auch zwei Gruppen gereicht“ (Thema: Ergebnissicherung) und sie greift eine Arbeitsphase auf, die sie im Nachhinein als wenig effektiv erlebt hat (Thema: Textbearbeitung).

In Bezug auf die Textarbeit spürt sie ein deutliches Unbehagen. Sie stellt fest, dass sie den Text hätte lesen und den Wortschatz klären müssen, denn ,im Nachhinein kam halt heraus ,puesto de trabajo und so, das war dann doch nicht klar“. Ihre Intervention „habt ihr das verstanden?“ und ,gibt es noch Fragen?“ erlebt sie im Nachhinein als wenig wirkungsvoll, da hätte sie „deutlicher nachfragen müssen“".

Alle die von ihr genannten Aspekte: Zeitdruck, Textarbeit, Ergebnissicherung und die Form ihrer Intervention hängen unmittelbar miteinander zusammen und lassen sich auf geringes Erfahrungswissen zurückzuführen. Schülerleistungen und Voraussetzungen richtig einzuschätzen und den Unterricht daraufhin $\mathrm{zu}$ planen, fällt ihr noch schwer, wie der folgenden Äußerung implizit zu entnehmen ist:

INA: ich hätte mir Zeit für die Zwischensicherung nehmen sollen, also gerade bei dem Text habe ich das gemerkt, obwohl ich den schon so konzipiert habe, dass er eigentlich einfach war und auch die Frage von der Sophia mit dem agitador, das war eigentlich genau das, was wir letzte Stunde vorentlastet hatten, aber da hätte ich mir eigentlich insgesamt mehr Zeit nehmen müssen, das fand ich jetzt selber nicht gut. [INA/1.UB/NB I: 36]

In der kritischen Auseinandersetzung mit ihrem Verhalten thematisiert sie vordergründig, dass sie sich nicht genug Zeit genommen habe, um den Text mit den Schülerinnen und Schülern zu besprechen. Dahinter verbirgt sich jedoch ihre Einschätzung, dass er, der Text, ,eigentlich einfach war" und daher für die Schülerinnen und Schüler leicht zu verstehen sein musste. Eine inhaltliche 
Sicherung hatte sie - wohl aus diesem Grunde - in ihrer Planung nicht vorgesehen. Zwar registriert sie in der Unterrichtssituation selbst Unsicherheit auf Seiten der Lerngruppe, sie vermag jedoch nicht, ihr Handeln unmittelbar anzupassen. Eine angemessene Intervention findet insofern hier nicht statt. In der Reflexion wird ihr nun im Nachhinein bewusst, dass sie sich für die Textarbeit hätte Zeit nehmen müssen.

Inwieweit Ina diesen fachdidaktischen Aspekt realisiert hat, macht sie zu einem späteren Zeitpunkt (2. UB) deutlich. Auf die Frage, was sie seit dem ersten Unterrichtsbesuch verändert habe, antwortet sie:

INA: ja, also Textarbeit war beim ersten Mal, glaub ich, Thema. Da habe ich keine Rückfragen zum Inhalt gestellt, das mache ich jetzt auf jeden Fall anders, ich hab immer eine inhaltliche Sicherung bevor ich dann darüber hinausgehend eine Frage stelle (...).

[INA/2.UB/NB II: 139]

Hier zeigt sich, wie sie eine Erfahrung aus dem ersten UB ,denn im Nachhinein kam halt heraus (...) das war dann doch nicht klar", und ihre Schlussfolgerung, „da hätte ich mir Zeit nehmen müssen“ in konkretes fachdidaktisches Handlungswissen überführt: „das mache ich jetzt auf jeden Fall anders, ich hab immer eine inhaltliche Sicherung, bevor ich dann darüber hinausgehend eine Frage stelle“.

\subsubsection{Der Faktor Zeit}

Ein weiterer Aspekt, der sich aus dem ersten UB und Inas Reflexion über die Wirksamkeit ihrer Intervention ergibt, hat mit dem Faktor Zeit zu tun.

So registriert Ina ein weiteres Mal, als sie die Stunde anhand des Videos (etwa eine Woche später) bespricht, dass ihre Form der Intervention, Schülerverständnis zu sichern, eine geringe Wirksamkeit hat. Es geht diesmal um die Initiierung einer Arbeitsanweisung:

INA: einmal bei 3 Minuten 40 und einmal bei 14 Minuten 30, da geht's beides Mal um eine Arbeitsanweisung (...) ich habe den Arbeitsauftrag (...) eben nur noch mal vorgelesen und hab dann vale? gefragt und dann kam natürlich nichts und habe dann einfach bien gesagt und hab weiter gemacht, das finde ich jetzt so im Nachhinein nicht so gut. (...) Vale finde ich so im Nachhinein so nichtssagend, ich hätte besser gesagt: gebt das noch mal mit eigenen Worten wieder oder: gibt es Fragen oder ich hätte speziell was fragen müssen, aber nicht dieses. ... Das ist so ein bisschen wie: alles klar? - ja, gut. Und da sind mit Sicherheit Schüler gewesen, denen nicht alles klar war, so. [INA/1.UB/NBII: 13-32]

Sie bemerkt, dass der Ausdruck vale wenig Aufforderungscharakter hat, wenn es darum geht, sicherzustellen, ob eine Anweisung von der Lerngruppe tatsächlich verstanden wurde. Das sei so „nichtssagend“, da hätte sie genauer nachfragen müssen, denn „da sind mit Sicherheit Schüler gewesen, denen nicht alles klar 
war“: Auch hier kommt ihr Selbstverständnis, dass alle Schüler in den Lernprozess einzubeziehen sind, zum Tragen und korreliert mit ihrer Beobachtung, wenig wirksam gehandelt zu haben.

Dass Ina sowohl den Text als auch die Arbeitsanweisungen nicht sichert bzw. dass sie diesbezüglich die Form der (Nicht-)Intervention als wenig effektiv erlebt, lässt sich aber nicht nur mit fehlendem Erfahrungswissen über Voraussetzungen von Schülerleistungen erklären. Auch der Zeitdruck, den sie empfindet, 'verführt' sie zu dieser Form der Flüchtigkeit, denn sie möchte die Stunde gemäß der Planung zum Abschluss bringen: ,ich wollte heute unbedingt noch dazu kommen, dass sie [die Schülerinnen und Schüler] irgendetwas präsentieren [INA/1. UB/NB I: 36].“

Was hier deutlich wird, ist etwas, das sich bei vielen Referendarinnen und Referendaren nicht nur zu Beginn der Ausbildung beobachten lässt und auf die Prüfungs- bzw. Bewertungssituation zurückzuführen ist: So stehen die Lehrpersonen in den 'Lehrproben' unter dem Druck, die Stunde gemäß des Stundenentwurfs durchzuführen und zu beenden, um zu demonstrieren, dass sie fähig sind, Planung und Durchführung aufeinander abzustimmen. Schülerreaktionen werden in kritischen Situationen zwar wahrgenommen, doch fällt es in der Regel schwer, von der ursprünglichen Planung abzuweichen. Hinzu kommt, dass die Erfahrung fehlt, angemessen einschätzen zu können, wie viel Zeit Schülerinnen und Schüler für einzelne Arbeitsphasen tatsächlich benötigen. Ina macht in dieser Stunde also 'Tempo', sie setzt sich und die Schülerinnen und Schüler unter Druck. In der abschließenden schriftlichen Reflexion resümiert sie dazu Folgendes:

Wie sich sowohl in der Nachbesprechung als auch bei der Videoanalyse gezeigt hat, neigte ich dazu, die Schülerinnen und Schüler unter Zeitdruck zu setzen. Daraus resultierte, dass die gut gestalteten Materialien an manchen Stellen „zu kurz" kamen. So wurde beispielsweise der Text (...) nicht hinreichend mit den Schülerinnen und Schülern besprochen (...). [INA/PORT/1.UB: 12-20]

Interessant ist nun, dass sich für Ina in ihrem 2. UB das Zeitproblem noch einmal wiederholt, diesmal allerdings mit umgekehrten Vorzeichen. Hatte sie in ihrem ersten UB zu wenig Zeit, weil sie die Bearbeitungszeit für die Schülerinnen und Schüler zu knapp bemessen hatte, verfügt sie diesmal über ein Zuviel an Zeit:

INA: ich hatte das Gefühl, dass die das am Anfang viel schneller verstanden hatten, als ich das eingeplant hatte, ahm, irgendwann sagte auch die Johanna, (...) wir sind eigentlich durch, ja, das hat mich etwas verwundert, die waren recht schnell, und auch bei den Aufgaben, (...) ich hatte jetzt gedacht, da gebe es noch mehr Nachfragen das hat besser geklappt, als ich dachte, (...) ich hab zuhause überlegt, ob dieser Arbeitsauftrag klar ist, und hab gedacht, das würde länger dauern, bis die das verstanden haben, aber das hat irgendwie sehr schnell geklappt und deswegen hatte ich zum Schluss auch mehr Zeit als gedacht (...). [INA/2.UB/NB I: 28$]$ 
Offensichtlich ist auch hier wiederum, dass es ihr (noch) an Erfahrung fehlt, um richtig einschätzen zu können, wie viel Zeit die Schülerinnen und Schüler für die Bearbeitung der Aufgaben benötigen. Zugleich vermag sie nicht in dieser Situation eine adäquate Handlungsanpassung vorzunehmen. Sie schiebt zwar am Ende der Stunde eine ungeplante Phase nach:

INA: (...) und die letzte Phase, das ist keine Frage, die ist aus der Not heraus entstanden, weil ich zu viel Zeit hatte. Ganz klare Sache. [INA/2.UB/NBI: 96]

Doch erlebt sie diese im Nachhinein anhand der Videoreflexion als wenig effektiv:

INA: das war das zum Schluss, wo ich auf einmal zu viel Zeit übrig hatte und dann selber Fragen gestellt habe, ist natürlich lehrerzentriert, keine Frage (...). [INA/2.UB/NB II: 55]

\subsubsection{Adaptive Lehrkompetenz}

Inwieweit es Ina schließlich gelingt, Situationsmomente in der Unterrichtsplanung zu antizipieren sowie in der Unterrichtssituation selbst ihr Handeln einer neuen Situation anzupassen, falls dies erforderlich ist, macht sie in ihrem 5. UB deutlich. Sie kommentiert z.B. die Textarbeit in der Nachbesprechung (I) mit folgenden Worten:

INA: (...) zu der zweiten Phase, wo es um die, ja Erarbeitung des Modelltextes ging, die hat meiner Ansicht nach sehr gut funktioniert, der Modelltext war gut gewählt mit den neuen Strukturen, sprich: dem periphrastischen Futur und dieser Modelltext ist meiner Ansicht nach auch gut zum Tragen gekommen, indem eben die Schüler Fragen an diesen Text gestellt haben, das setzte dann eben auch ein Textverständnis voraus und offensichtlich waren sie auch in der Lage das zu lösen, das heißt, sie haben den Text auch verstanden (...). [INA/5.UB/NB I]

Zufrieden stellt Ina fest, dass der Modelltext gut gewählt und gut zum Tragen gekommen sei. Die Schülerinnen und Schüler „haben den Text auch verstanden“, da sie den Arbeitsauftrag, Fragen an den Text zu stellen, ohne erkennbare Schwierigkeiten lösen konnten. Ina teilt hier implizit mit, dass sie inzwischen sehr wohl einschätzen kann, ob ein Text dem Lernstand ihrer Lerngruppe angemessen ist, bzw. dass sie ihren Unterricht auf zu erwartende Schülerreaktionen und -leistungen abzustimmen und auch Unterrichtssituationen im Nachhinein (wiederum auf der Grundlage von Schülerreaktionen und -ergebnissen) richtig einzuschätzen und zu beurteilen vermag.

Auch in Bezug auf mögliche Schwierigkeiten, die die Lerngruppe mit einer Aufgabe haben könnte, vermittelt Ina, dass sie diese im Vorfeld entsprechend zu antizipieren vermag. In der Nachbesprechung (I) erklärt sie z. B., dass eine Bildbeschreibung für Lernerinnen und Lerner im ersten Lernjahr schwierig sei, 
INA: (...) in der ersten Phase als es um die Bildbetrachtung ging, war es mal wieder etwas schleppend, das hatte ich allerdings schon, ja, vorausgeahnt, (...) ich glaube, dass es sehr schwierig ist für Lerner gerade im ersten Lernjahr (...). [INA/5.UB/NBI: 15]

da sie die Bildbeschreibung für eine anspruchsvolle kommunikative Aufgabe halte. Sie erwartete daher nicht, dass die Schülerinnen und Schüler sich unmittelbar äußern könnten und räumte ihnen eine entsprechende (Denk-)Zeit ein.

(...) ich hab auch diesmal ihnen erst Zeit eingeräumt und habe nicht direkt ... ja, direkt Rückmeldung von Schülerseiten erwartet (...). [INA/5.UB/NBI: 15]

Dass es Ina nicht nur gelingt, Situationen oder Schwierigkeiten in der Unterrichtsplanung zu antizipieren oder Situationen im Nachhinein adäquat zu beurteilen, sondern dass sie auch während des Unterrichts zu reagieren vermag, wenn eine Handlungsanpassung erforderlich ist, auch das vermittelt Ina in der Nachbesprechung zu ihrer Stunde:

INA: Dann zum Schluss noch eine Bemerkung zu dem sistema formal (...) ich habe mich dazu entschlossen, anders als es eigentlich geplant war (...) doch ganz kurz wenigstens eine Sache aufzugreifen, die mir während des Omniumkontakts beim Beobachten der Schüler aufgefallen ist, nämlich, ja, die Verneinung. Wie verneint man beim unmittelbaren Futur, wo gehört das Verneinungswort „no“ hin, das habe ich bei ganz vielen gehört, dass das eben falsch war, dass sie das vor den Infinitiv gesetzt haben und von daher schien mir das sinnvoll, damit sich das nicht verfestigt in ihren Köpfen, das aufzugreifen und kurz zu thematisieren (...). [INA/5.UB/NB I: 15]

Als sie während des Unterrichts feststellt, dass die Lerngruppe Schwierigkeiten mit der Verneinung im Spanischen hat, weicht sie von ihrer ursprünglichen Planung ab und schiebt am Ende der Stunde eine Korrekturphase ein. Hier zeigt sich ganz deutlich, dass ein Lernprozess in Bezug auf eine adaptive Lehrkompetenz stattgefunden hat. Sie nimmt während des Unterrichts ein Problem wahr und vermag unmittelbar mit einer didaktischen Maßnahme darauf zu reagieren.

Auch im Umgang mit der zur Verfügung stehenden Zeit, scheint Ina einen echten Lernprozess erfahren zu haben. So nimmt sie in der Videoaufzeichnung (NB II) an sich ein verändertes, entspanntes Verhalten wahr und stellt anerkennend fest:

INA: (...) man sieht wirklich, die Beteiligung ist wieder sehr gering und, ja, positiv fand ich jetzt hier an meinem Verhalten, dass ich warte, ich halte sozusagen die Stille aus, das fand ich schon ganz gut (...). [INA/5/NBII: 10]

Sie bewahrt Ruhe, hält die „Stille“ aus, obwohl diese Anlass geben könnte, zu intervenieren. Abwarten, Schülerinnen und Schülern Zeit geben, Situationen zulassen, so könnte die Erkenntnis lauten, die sie aufgrund vorangegangener Erfahrungen gemacht hat. Ihrem Verhalten liegt Bewusstheit zugrunde: Sie reagiert auf die Schülerinnen und Schüler, indem sie - statt in Aktionismus zu verfallen - die Stille bewusst aushält. 


\section{$6 \quad$ Fazit}

Zusammenfassend lässt sich festhalten, dass sich auf der Grundlage der Nachbesprechungen (I und II) am Beispiel der Referendarin Ina ein Lernprozess im Sinne einer adaptiven Lehrkompetenz nachzeichnen lässt. Sie kann Diskrepanzen zwischen ihrem professionellen Selbstkonzept und ihrer Selbstwirksamkeit verringern, indem sie ihr neues Erfahrungswissen angeleitet durch die Reflexionsgespräche und den Videoimpulsen in ihr professionelles Selbstkonzept und schließlich auch in ihr handlungsleitendes berufliches Selbstvertrauen überführt. Selbstkritisch beobachtet und reflektiert Ina ihre Wirksamkeit in Bezug auf das Unterrichtsgeschehen. Dabei vermittelt sie in den Gesprächen implizit, dass sie sich zunehmend in der Lage sieht, nicht nur den (individuellen) Lernstand ihrer Schülerinnen und Schüler zu antizipieren und ihre Planung angemessen darauf abzustimmen, sondern dass sie zugleich auch während des Unterrichts Handlungsanpassungen vorzunehmen vermag, wenn eine Situation dies erforderlich macht. In und durch ihre Reflexionen vermittelt Ina eindrucksvoll, dass ein gezielter Selbstlernprozess stattgefunden hat. Der bewusste und reflektierte Umgang mit den im Unterricht praktizierten und in der Retrospektive wahrgenommenen und thematisierten Handlungen haben in ihrem Fall zu einem sukzessiven Kompetenzaufbau im Sinne einer adaptiven Lehrkompetenz geführt. Es lässt sich insofern hier nachverfolgen, wie sich Erfahrungswissen und die jeweilige reflexive Verarbeitung in einem zunehmenden praktischen Handlungswissen manifestiert.

\section{Bibliographie}

Abendroth-Timmer, Dagmar (2011). Reflexive Lehrerbildung: Konzepte und Perspektiven für den Einsatz von Unterrichtssimulation und Videographie. Zeitschrift für Fremdsprachenforschung 22/1: 3-41.

Abendroth-Timmer, Dagmar / Frevel, Claudia (2013). Analyse handlungsleitender Kognitionen anhand videogestützter Reflexionsprozesse angehender Spanischlehrender in verschiedenen berufsbiographischen Kontexten. In: Riegel, Ulrich / Macha, Klaas (Hrsg.): Videobasierte Kompetenzforschung in den Fachdidaktiken. Münster, New York, Berlin: Waxmann. 133-149.

Altrichter, Herbert / Posch, Peter (1998). Lehrer erforschen ihren Unterricht. Eine Einführung in die Methoden der Aktionsforschung. Bad Heilbrunn: Klinkhardt.

Beck, Erwin / Baer, Matthias / Guldimann, Titus / Bischoft, Sonja (2008). Adaptive Lehrkompetenz, Analyse und Struktur, Veränderbarkeit und Wirkung handlungssteuernden Lehrerwissens. Münster, New York, München, Berlin: Waxmann.

Berliner, David C. (1992). The Nature of Expertise in Teaching. In: Oser, Fritz / Dick, Andreas / Patry, Jean-Luc (Hrsg.): Effective and Responsible Teaching. The New Synthesis. San Francisco: Jossey-Bass. 227-248. 
Bromme, Rainer (1992). Der Lehrer als Experte. Zur Psychologie des professionellen Wissens, Bern: Huber.

Caspari, Daniela (2003). Fremdsprachenlehrerinnen und Fremdsprachenlehrer. Studien zu ihrem beruflichen Selbstverständnis. Tübingen: Narr.

Frevel, Claudia (2011). Über Unterricht reden. In: Claudia Frevel / Franz-Josef Klein / Carolin Patzelt (Hrsg.): Gli uomini si legano per la lingua. Stuttgart: ibidem. 525-547.

Groeben, Norbert / Wahl, Diethelm / Schlee, Jörg / Scheele, Brigitte (1988). Das Forschungsprogramm subjektive Theorie. Eine Einführung in die Psychologie des reflexiven Subjekts. Tübingen: Francke.

Helmke, Andreas (2009). Unterrichtsqualität und Lehrerprofessionalität, Diagnose, Evaluation und Verbesserung des Unterrichts. Seelze-Velber: Klett-Kallmeyer.

Herzog, Walter (2003). Zwischen Gesetz und Fall. Mutmaßungen über Typologien als pädagogische Wissensform. Zeitschrift für Pädagogik 48: 383-399.

Herzog, Walter (2011). Professionalität im Beruf von Lehrerinnen und Lehrern. In: Berner, Hans / Isler, Rudolf: Lehrer-Identität, Lehrer-Rolle, Lehrer-Handeln. Hohengehren: Schneider Verlag. 49-77.

Krewer, Bernd / Eckensberger, Lutz H. (1998). Selbstentwicklung und kulturelle Identität. In: Hurrelmann, Klaus / Dieter, Ulrich (Hrsg.): Handbuch der Sozialforschung. Weinheim u. a.: Beltz. 5. neu ausgestattete Aufl. 573-594.

Mead, George Herbert (2005). Geist, Identität und Gesellschaft aus Sicht des Sozialbehaviorismus. Frankfurt/M.: Suhrkamp.

Messner, Helmut / Reusser, Kurt (2000). Die berufliche Entwicklung von Lehrpersonen als lebenslanger Prozess. Beitrgäge zu Lehrerbildung 18/2, 157-171.

Schocker-v. Ditfurth, Marita (2001). Forschendes Lernen in der fremdsprachlichen Lehrerbildung. Grundlagen, Erfahrungen, Perspektiven. Tübingen: Narr.

Schön, Donald A. (1983). The Reflective Practitioner: how professionals think in action. London: Temple Smith.

Schüpbach, Jürg. (2007). Über das Unterrichten reden. Die Unterrichtsnachbesprechung in den Lehrpraktika - eine Nahtstelle zwischen Theorie und Praxis? Bern: Haupt Verlag. 


\title{
English as a Lingua Franca and the Envisioning of Pre- service English Language Teacher Education in the $21^{\text {st }}$ Century: Narrative Insights from İstanbul and Berlin
}

\author{
Özlem Etuş / Katrin Schultze (Istanbul, Berlin)
}

Der im Zuge der Globalisierung kontinuierlich steigende Stellenwert von Englisch als lingua franca (ELF) verlangt nach einem präziseren didaktischen Verständnis der lokalen und globalen Mechanismen, die im Bereich der Fremdsprachenlehrer(innen)bildung in Expanding Circle-Regionen wirken. Dieser Aufsatz präsentiert die Ergebnisse einer komparativ angelegten qualitativ-empirischen Studie, die anhand von Sprachlernbiografien, Einzel- und Gruppeninterviews den Zusammenhang von ELF und den professionellen Identitäten von Englischlehramtsstudierenden aus İstanbul und Berlin untersucht. Als methodologische Basis dienen Alvesson und Sköldbergs „Reflexive Methodology” (2009), Barhuizens Konzept des „Narrative Knowledging” (2011) sowie die „Grounded Theory Method" nach Strauss/Corbin (1996). Abschließend werden Zukunftsperspektiven und Aufgaben für die Englischlehrer(innen)bildung in ELF-Kontexten aufgezeigt.

Milletler-ötesi akışlar ile tanımlanan bu çağda, lingua franca olarak İngilizce'nin değişen konumu ve bunun eğitime yansımaları, İngilizce'nin uluslararası dil olarak kullanıldığ1 ortamlarda öğretmen yetiştirme sürecini etkileyen yerel ve küresel oluşumların derinlemesine incelenmesini gerektirmektedir. Bu çalışma, İstanbul ve Berlin şehirlerinde bulunan iki devlet üniversitesinde eğitimlerini sürdüren İngilizce öğretmen adaylarının lingua franca olarak İngiliz diline bakışını ve gelecekteki eğitmen kimliklerini bununla ne ölçüde bağdaştırdıklarını karşılaştırmalı bir yaklaşım ile irdelemeyi ve bu doğrultuda İngilizce'nin lingua franca olarak kullanıldığı iki ayrı ortamda yürütülmüş olan uluslararası bir araştırma projesinin bulgularını paylaşmayı amaçlamaktadır. Bu niteliksel araştırmada, dil öğrenim geçmişleri, bireysel görüşmeler ve odak grup görüşmeleri yoluyla elde edilen anlatı bütüncesinin incelenmesi sürecinde Alvesson ve Sköldberg'in "Reflexive Methodology" (2009), Barkhuzian'1n “Narrative Knowledging" (2011) ve Strauss ve Cobin'in "Grounded Theory Method" (1996) kuram ve yöntemlerinden faydalanılmıştır. Sonuç bölümünde, İngilizce’nin küresel erişiminin lingua franca ortamlarındaki öğretmen eğitimi sürecinde ne tür gelişmelere yön verebileceği tartışılmaktadır.

\section{$1 \quad$ Introduction}

Today, English as the world lingua franca possesses a status which has rarely, if ever, been reached by any other language in history. It dominates the scene as the language of media, communication technology, business and academia in various instances of transnational encounters. However, as Seidlhofer (2011: 15) discusses, despite the emergence of English as a lingua franca (ELF) - which can generally be understood as English used among speakers of different first languages - what the word 'English' actually stands for remains unchallenged, 
as standard language ideology and Inner Circle norms continue to pervade English language teaching. In her words: "English is English is English" (ibid.: 11).

The boundaries of English language use in Inner, Outer, and Expanding Circles, originating from Kachru's (1992) model, fall short to conceptualize the de-territorialized status of English as a global language. According to Berns (2005: 85), the lines between Outer and Expanding Circles become increasingly blurred and the Expanding Circle comes to represent "the rest of the world", with a growing number of people using English for a variety of purposes in various contexts. In an era marked by transnational flows and new varieties of English, it is no longer possible to define the language by geographical locations and to make a clear distinction between 'native' and 'non-native'. In a similar vein, Yano (2009) questions the issue of "connecting native English speakers exclusively to the Inner Circle" (ibid.: 247), when "the native-speaker's normproviding role, the second language speaker's norm-developing trend, and the foreign language speaker's norm-dependent nature are gradually changing" (ibid.: 248).

The emerging reality of ELF calls for a reconceptualization of the English language and the way it is being taught, and hence necessitates the empirical investigation of its possible implications for language teacher education in lingua franca settings. The article attempts to respond to that need by presenting the findings of a bilateral research project, which investigates how the processes of globalization and the status of English as a world language influence the professional identity development of prospective English teachers in two lingua franca contexts, namely Berlin and İstanbul. The research focuses on three interrelated questions: How do prospective English teachers relate to ELF in terms of their own language learning experiences? What are their beliefs and attitudes about ELF? To what extent do they envision a transition towards more ELF-sensitive teaching methodologies for the future?

Being confined to the local contexts of two state universities' ELT departments, the research findings cannot be assumed to be representative of larger populations, cultures, or nations, but - in a non-prescriptive comparative perspective - they may pave the way for new insights, emerging issues and reconceptualizations in the field and help us to seek future possibilities for language teacher education. In agreement with Adamson (2012: 647), such studies can "bring [one's own] findings into sharper relief through a conscious decision to use comparison and contrast", but they cannot and should not claim equal validity for both sides: As he puts it, "[C]omparative education is very much about the betterment of our own society rather than the somewhat patronising or hegemonic imposition of our own policies and practices on other societies" (ibid.). 


\section{Research methodology}

\subsection{Data collection}

The driving motive of this study was to explore the similarities and differences in experiences, attitudes and perspectives across the two contexts, and to capture unique personal responses, which might have wider implications for ELFsensitive discussions. Therefore, adhering to an "emergent research design" (Dörnyei 2007: 37), various resources including language learning histories (LLH), transcripts of one-to-one and focus group interviews, field notes and memos were collected in both research settings between October 2011 and March 2012. The dynamics of each local context is unique, and therefore the coresearchers set about achieving parallelism in research composition, but refrained from following a strict comparative research frame for data collection and analysis.

The narrative data collected in the first phase of research comprised a total of 68 LLHs (45 from İstanbul and 23 from Berlin), which were written by volunteer graduate and undergraduate students enrolled in the ELT departments of the two universities. The texts were generated by the question how the participants became the speakers of English they were at present. In Berlin, the participants were asked to write their stories in English (with the option to add details or comments in German), in İstanbul, the choice of language was left open to the students, but nearly all chose to write in English.

In the second phase of research, the database was complemented by oral and co-constructive narratives attained from one-to-one and focus group interviews. The interviews in both settings were conducted in the native language, transcribed and translated to English. In İstanbul, data was collected in recurrent sessions of focus-group interviews with four different groups $\left(3^{\text {rd }}\right.$ grade, $4^{\text {th }}$ grade, MA and $\mathrm{PhD}$ ), each consisting of four to six participants with a total population of 18 , comprising 20 hours of recording. The process of data collection in Berlin moved along a similar line: three focus group-discussions with BA and a fourth focus group discussion with MA students were conducted, involving 12 participants and amounting to five hours of recording time. Instead of repeated group sessions, four one-to-one interviews (with two BA and two MA students, yielding four hours of recorded data) were added to extend the inquiry, thus favouring the individual perspective over the co-constructive.

A semi-structured interviewing style was adopted. Some of the key topics and categories which emerged in the analysis of the language learning histories such as 'going abroad', 'globalization', 'speaking English', 'native speakerism' and 'the status of English as a world language' - were written on index cards and used to stimulate response in an open-ended and flexible manner. In addition, related prompt questions on ELF concerns - for instance how the 
participants conceptualize English, if they see any potential for adopting ELF approaches in language education, and how they envision ELF teacher qualifications both on a local and global scale - were used to generate further elaboration on answers and give direction to verbal interaction. There was minimal interfering in the interviewing process, yet co-researchers agreed that full scientific neutrality would neither be the case nor a desired goal. Rather, in agreement with Fontana/Frey (2005), the interview phase was regarded as "an active emergent process" (ibid.: 706), in which "interactions between two (or more) people [were] leading to negotiated, contextually based results" (ibid.: 698).

\subsection{Data analysis: practical and theoretical considerations}

The phase of data analysis posed a series of thought-provoking questions: How to make meaning of the extensive and highly complex narrative data collected in two different local contexts by parallel, yet not completely identical methods of data collection? How to give the analysis structure and depth? How to expand on the interpretation and reach shared conclusions considering the varying backgrounds of the two researchers?

First of all, the co-researchers' shared interest in professional identity formation, inspired by Norton's (2000: 5) definition of identity as "how a person understands his or her relationship to the world, how that relationship is constructed across time and space, and how that person understands possibilities for the future", offered them a research framework based upon the three interlinked dimensions of time - past, present and future - thus orienting the analysis towards three major topics: the participants' ELF experiences, their attitudes and beliefs about ELF, and their future perspectives with regard to ELF.

Secondly, Alvesson/Sköldberg's "Reflexive Methodology" (2009) was utilized to guide the analysis and obtain a multi-layered and holistic view of the qualitative data. The authors offer a helpful "quadri-hermeneutics" (ibid.: 271), a meta-theoretical reflexive framework that works on four different, yet intertwined levels of interpretation, based on the epistemological premises of four major currents of methodology and philosophy of science. Accordingly, the interpretation on level 1 is indebted to the tradition of empirically oriented currents such as Grounded Theory: it is data-driven and aims at acquiring good knowledge of the material. The interpretation on level 2 is rooted in Hermeneutics and is insight-driven, attempting to develop a more profound and meaningful interpretation. Level 3 is the level of Critical Theory and thus emancipation-driven, placing particular emphasis on the wider contexts and social processes linked to the research project. The fourth level of reflexion is based on Postmodernism and polyphony-driven; it considers issues such as 
authorship, authority and representation and focuses on the multiple voices involved in the research (ibid.: 283-285).

On level one, the LLHs and interviews were analysed by Grounded Theory, particularly by the open and axial coding procedures developed by Strauss and Corbin (1996). The data was broken apart into manageable chunks, preliminarily labelled and put together in a new way (cf. Strauss/Corbin 1996: 39) to generate codes and categories in an attempt to identify the topics and processes which were relevant to the participants' ELF experiences as learners and users of ELF. (e. g. stays abroad, communicative activities at school). However, as Alvesson/Sköldberg (2009: 68) argue, a possible disadvantage of the Grounded Theory coding process is that "real organic relations between the incidents are broken", confining the analysis to how people "perceive individual notes ('incidents')" at the expense of missing "the essential element - the melody". Therefore, the data-driven analysis was enriched by methodological strategies deriving from Qualitative Content Analysis: to this end, "progressive focusing" (Dörnyei 2007: 132), in which the flexible and cyclic processing of data is accompanied by memos involving reflections on the circumstances of data collection and analysis and real-time comments on the participants' actions, behaviours and discursive choices during the interviews, was used to expand the interpretation repertoire of the researchers.

The cyclical analysis of data gradually shifted the investigation from level 1 to level 2 and enabled a more insight-driven focus: the emergent categories derived from the analysis of LLHs helped the researchers to explore the interconnectedness between certain themes, e. g. the participants' attitudes to English and to the English language teaching profession, and to utilize these key points in interviews to generate further discussion points, such as perceptions of proficiency in English or the scope for ELF teaching in the future. Moreover, the focus group discussions allowed the participants to share ideas and experiences, occasionally challenge or support each other, but above all, they involved them in the negotiation and reconstruction of meaning. A more context-sensitive and holistic approach to the narrative data was achieved by a heightened interest in "small stories". Vásquez (2011), who differentiates between "big stories" attained from autobiographies and "small stories" unveiled in everyday conversational contexts, claims that new insights might be attained in identity sensitive narrative research by focusing on the latter, as the analysis of "narrative in context" offers further perspectives in the way participants reevaluate, negotiate and co-construct their ideas in the process of social interaction. Citing Freeman's (2007: 156) emphasis on emergent small stories in "everyday, non-interviewed life" as an object of narrative inquiry, Vásquez (2011: 539-540) argues that narratives emerging from (focus group) interviews are also types of sociocultural practices, in which the roles of researchers and the interaction between participants are accounted for in discussing not only the 
What's, but also the How's of the meaning making process. Following a hermeneutic interpretation strategy, along with the analysis of content, researchers also tried to attend to the stylistic aspects of the narratives such as the use of irony, metaphors and imagery to unravel the more hidden meanings of the data.

Through the small story narrative analysis, the focus of interpretation was shifted to level 3, as the macro contexts of the two Expanding Circle research settings were subjected to critical scrutiny, e. g. the status of English as a global lingua franca, the underlying socio-political processes and ideologies operating in English language education, and the related expectations of the teachers by society and educational institutions.

Postmodern reflective strategies were utilized on level 4 by taking into account the asymmetrical relationship between researchers and participants deriving from their roles as lecturers and students. Researchers tried to stay alert to possible effects of dominance and authority (e. g. social desirability bias), to be reflexive about their own research experience and problematize their initial interpretations to "highlight ambivalences and the potential for alternative representations" (Alvesson/Sköldberg: 276). In this vein, Barkhuizen's (2011: 57) concept of "narrative knowledging" proved to be inspiring as it signifies a process of knowledge production which involves all stages of narrative building, starting from the knowledge constructions of the participants to the reconstructions of this meaning-making process by the researchers. By looking at the data through the lens of Barkhuizen (2011), researchers were constantly reminded of the plurality of voices involved in the analytical process, of the different dimensions of time and of the uniqueness and contingency of each narrative act. Regular researcher interchanges became an important part of the project's narrative knowledging process, and as an attempt towards "investigator triangulation" (Mackey/Gass 2005: 181), a randomly selected portion of data was cross analysed to achieve interim results.

\section{Discussion of research findings}

The theoretical frame presented in the previous section offered a comprehensive picture of what ELF means to future English teachers from İstanbul and Berlin by reporting on three major concerns: the participants' experiences with ELF, their present attitudes and beliefs with regard to ELF and their expectations and aspirations towards a future of learning and teaching ELF. 


\subsection{ELF experiences}

The research findings reveal that the range of first-hand ELF experiences differs from one Expanding Circle context to another. The LLHs collected in İstanbul give evidence of the participants' very limited ELF experiences throughout their education. Very few students gathered ELF experiences outside the country, either in ESL settings or through contact with native and/or non-native speakers in other Expanding Circle countries. Their ELF experiences within the country were also limited, not going beyond a few interactions with tourists, visiting students from other countries, and the children of relatives who migrated to Inner Circle countries. In their discussions, they mentioned increased opportunities for ELF experiences, largely achieved through social network sites such as Facebook, Skype, blogs and chat rooms, but as they noted, such contacts were still not part of their everyday communication. Outside the country, ELF encounters had been possible for a few participants who found the opportunity to travel by means of Erasmus, work \& travel, and youth programmes.

Almost every LLH from Berlin entailed at least one account of a first-hand lingua franca experience and in most cases these experiences were presented as key events in the authors' language learning processes. The students' ELF experiences ranged from family holidays in Denmark or Greece to high-school years in the United States or Australia to work experiences in international companies in Italy or Germany. Many of them were part of the participants' private lives (e.g. holidays, hobbies, English-speaking partners or family members), but even more were linked to their professional selves as high-school students, university students or employees (e. g. class trips, high school years abroad, Au-pair, Erasmus, Work \& Travel, internships). Most of the latter experiences took place in semi-institutional frameworks, i. e. student exchange programmes offered by various public or private agencies, sometimes at considerable costs.

A second major difference between the participants' ELF-related experiences lies in the different teaching cultures they experienced at school. The participants in İstanbul storied a strong orientation to form-focused teaching with a low tolerance for errors, which - in their view - had limited their opportunity to develop communication skills. The participants offered many reasons for this situation: learning English with teachers who majored in other subject areas such as mathematics, social sciences, and history was a common experience cited in narratives and as some participants reasoned, lacking the required competence in speaking, grammar and vocabulary turned out to be 'safe havens' for these teachers. However, as others argue, the experiences of the ones who were taught by, in their terms, 'real' English teachers were not so different: these teachers either lacked the required skills to conduct communicative lessons or were strictly obliged to follow the syllabus and the 
materials which always take the 'standard language' as the norm. For these students, the traditional approach to language teaching was largely taken due to socio-political processes governing the educational system in Turkey: entry to secondary and tertiary education is regulated through centralized exams, which necessitate an extensive focus on standard-driven language competency in order to attain better results in multiple-choice exams.

The narratives from Berlin conveyed a picture of a communication-oriented teaching culture with a considerable range of communicative activities, such as singing, role playing or project work. Often, the participants' favourite teachers were those who chose learner-oriented topics, encouraged autonomous learning and tried to integrate 'authentic' communicative settings into the classroom. There was, however, a noticeable difference between primary and secondary school experiences: in many stories, this transition went along with a setback from highly communicative to far more grammar-focused teaching methods, and not rarely did participants talk of a 'grammar shock' when entering high school.

The following section discusses if and to what extent these early language learning experiences shape the participants' attitudes and beliefs about ELF.

\subsection{Attitudes and beliefs about ELF}

Conceptualizing English

Although former language learning experiences showed a great difference in terms of first-hand ELF encounters and teaching methodologies experienced at school, a close analysis of participant narratives revealed a shared finding: a general lack of awareness and uncertainty in defining what ELF stands for.

Nearly half of the participants in İstanbul acknowledged the status of English as a world lingua franca, but still displayed an orientation towards Englishspeaking cultures in the Inner Circle, and revealed a strong attachment to a native speaker 'ideal' when defining competency in language use. As also discussed by Seidlhofer (2011: 14), ELF is recited in many contexts, but "[it] has not really entered people's consciousness as a new and alternative concept of "English", leading on to a "conceptual gap". Focus group discussions with student teachers in İstanbul showed that this conceptual gap cannot easily be abridged without a clear definition of the function of English in their lives. While voicing out the importance of having a need-oriented conceptualization of English, they also claimed that an encompassing view on English cannot be offered when the needs, the language itself and the functions it performs are constantly changing. In the following extract from a focus group discussion, a prospective teacher pointed out the existence of different 'Englishes' at different stages of his life: 
First English was my toy something to play with, something to discover when I was a little boy, then during my school years it became a subject to be mastered along with other subjects and then it gradually turned out to be a mission, even an enemy to be defeated in the exams in order to secure a place in higher education. As I am just saying to myself that everything is $\mathrm{Ok}$, it is a friend again which will accompany me in my future professional life, nowadays, it is again becoming a new mission for me, something I should be learning again because in the call centre where I have a part time job it takes good three minutes for me just to get the credit number of someone who calls from Ireland, Wales or any other place [italics added for emphasis] (FG $4^{\text {th }}$ grade/2, 29.11.2011, 18:40).

Intrinsic motivation to "discover" the language has played a role only at his early ages. His metaphorical conceptualization of English as "subject", "mission", "enemy", and "friend" indicate that his contact with English is closely bound up with his institutional identities where English is a key to achieve success in academic and professional life. While giving merit to the communicative benefit of English in other parts of his talk, the extract shows how difficult it is to offer a new conceptualization of English when his experience with language is largely governed through socio-economic and institutional forces.

The overall picture drawn from the analysis of data collected in Berlin also revealed a lack of awareness and difficulty in defining ELF, which is vaguely associated by many participants with "an English which is spoken all over the world" or "an English which is spoken with many mistakes" (FG MA, 12.03.2012; 11:15). In the course of the interviews, however, some participants specified their first definitions, e. g. by "the best common language basis/the best common linguistic ground for speakers from different countries" (FG MA, 12.03.2012; 11:18). The latter, however, usually did not include Inner Circle speakers. The texts contain a considerable amount of ELF experiences in Outer and Expanding Circle countries with occasional references to the value of these experiences. As one participant comments, her Erasmus stay in Spain made her "lose all the embarrassment that [she] had felt before, when visiting English speaking countries" (FG MA, 12.03.2012; 11:28). Most participants in Berlin portray encounters with ELF speakers as entertaining and motivating experiences, but do not consider them beneficial for 'real' or 'proper' learning. One student, for instance, reports in his language learning history that he worked in a restaurant in Canada together with people from Haiti and Jamaica, but concludes: "In terms of language learning the place was not too helpful. The way in which the crew communicated with each other would have made the toughest sailor soil himself." (LLH B03_2011). The comment shows that this particular lingua franca setting is perceived as a source of bad influence rather than an enriching resource for language learning. 
Attitudes towards English language learning and teaching

The participants' attitudes and beliefs towards learning English are almost as much dominated by native speaker norms and Inner Circle standards as their language learning experiences in both research settings, yet in different ways and with different underlying reasons.

Participants in İstanbul expressed their aspirations for more communicative methodologies, but a reconceptualization of language teaching decentred from Inner Circle Englishes was not seen as a future possibility. A focus group interview with five student teachers offered an instance of fragmented and conflicting views on English teaching with close reference to political, sociocultural and educational underpinnings. One participant's insistence on teaching American English due to Turkey's close relation with the United States was negated by another participant who claimed that British English would continue to be the focus of interest, considering the larger share of British publishing houses on the market. Another participant displayed an awareness of Englishes, while seeing the need for "a reference point, a centre from which other decentralized forms would be emerging and ELF is there to bring a balance to this kind of divergence" (FG $4^{\text {th }}$ grade/2, 29.12.2011, 18:49). This view was challenged by another interviewee who claimed that language change and consequent "deviation from the original" had always been a natural process of any language throughout human history, whether generated by native or nonnative speakers. He, therefore, problematized any kind of "ownership", including an ELF-centred one. This critical stance towards ELF was supported by his peer who rejected all kinds of "standardization" (FG $4^{\text {th }}$ grade/2, 29.12.2011, 18:53). These discussions indicate that any understanding of ELF needs to be negotiated, supported and challenged, but eventually co-constructed by future practitioners if it is to become a more concrete entity with practical implications for teaching.

In a few participants' narratives, a change in perspective from an excessive focus on Inner Circle Englishes to the discovery of ELF concerns was discernible. The following remark of a graduate student is significant as it shows how past experiences in language learning might pave the way for present practices in teaching and offer projections for the future in becoming a professional:

At first the course books, audio visual supplementary materials and our teacher provided us an Anglophonic English. The closer we get to the British English, the better performance we proved to have. Almost mimicking the British standards in terms of articulation, grammar or daily communication was compulsory [...]. Today my tendency towards EFL standards are more flexible as the post-modern product, Global English highlights the golden 'Let it pass' rule to prioritize the cultural assets of non-native interlocutors with the unique aim of mutual intelligibility [...]. Yes we still take care of the linguistic norms but, if the message of my student is clearly conveyed, I do not play 
the Prince to correct any mistake. [...] In other words my students do not have to 'have butterflies in their stomach' but can easily feel nervous as if they are going to faint.

The data collected in Berlin also contained critical remarks about institutional language learning experiences, but these did not go beyond individual criticisms diverted to particular teachers, who treated their students unfair or offered "boring" lessons (LLH B01_2011). Macro-level considerations on foreign language policies or teacher education were not part of their arguments. Moreover, the LLHs revealed a strong belief in the strategy of immersion and agreed upon the idea that 'real' English learning can only happen abroad, in Inner Circle countries. Interestingly, in several narratives, after a considerable time spent with native speakers in countries such as Canada, the USA or the UK, starting to "dream in English" is conceived as a sign of success in language learning. As one participant recalls her experiences in the USA:

And that was the most important factor for learning the language: The everyday practice! I didn't learn English anymore, I was using it on a daily basis. At first I felt very shy, I thought my vocabulary does not suffice and it took me too long searching for words. But through the practice I became more fluent and daring. I started to watch and understand whole movies in English, I could follow math-classes at school and then it happened: My first dream in English. I think that's the point where I realized that I now master a new language. It was a whole new feeling to it. English was not a conscious act anymore but it was now just there for me and I could submerge into the culture with ease. (LLH B20_2011)

By starting to dream in English, the learner seems to have adopted an entirely new identity, characterized by feelings of mastery and individual ownership, but at the same time by the desire to fully submerge into the foreign culture.

The various stories of immersion told by participants from Berlin also hint at the existence of a 'reproductive cycle' of Inner Circle orientation and native speaker norms encouraged by the German school system: spending a high school year abroad in an Inner Circle country for attaining native-like competence in language earns learners the appreciation and recognition of peers and teachers as well as far better grades upon returning to Germany, thus encouraging them to choose English as Leistungskurs (A-levels) and later in many cases to become English teachers, who again orient themselves, their teaching, and their students towards native speaker and Inner Circle norms (e. g. LLH B20_2011).

As the discussions in this section reveal, a sharp distinction exists in the way participants position themselves with respect to ELF concerns. In Berlin, it is rather indifference and a lack of awareness that cause the student teachers' reservations than any serious objection against the notion of ELF. The participants in İstanbul, on the other hand, offer a critical perspective of the macro dynamics of language and language teaching in local and global contexts when synergizing discussions on the pedagogy of ELF. In other words, the real 
issue for them is if, and to what extent, language teaching pedagogy can generate ELF methodologies when the system in Turkey prioritizes the standard language for assessment, evaluation and placement and takes 'native speakers' as norm-abiding? The situation of the two groups can be metaphorically conceptualized as 'comfort zone vs. battle-field' in terms of language learning and teaching, unveiling multi-fold implications for their professional identity construction.

\section{Identity issues}

Research findings indicate that student teachers might experience fragmentation in terms of foregrounding different identities depending on their positioning as language learners, ELF users and/or future professionals. In the following excerpt from a focus group interview, a German student teacher talks about her Erasmus stay in Spain and shows how multiple identities are being negotiated (all names are pseudonyms):

Mona: When I spent my Erasmus time [in Spain], I remember well, that all of us Italians, French people, Germans, and so on - that we all communicated with our pretty poor Spanish - which, of course, was not as good as English - and that many interference mistakes were made, especially by the Italians, but it was still possible to understand each other, somehow. The Italians simply translated all their Italian words into a Spanish pronunciation ...

Thea: ... they gave it a Spanish "touch" (laughs)

Mona: and then they think it's Spanish (all laughing). Well, but we somehow understood each other. But the same thing happens with English, if everybody uses it as a lingua franca, lots of mistakes come into the language, e.g. "isn't it" becomes something like "not?" or "no?", you know? (Helen: Umh) So it somehow again becomes a differrent kind of English, which doesn't raise this claim to correctness anymore, but gives most importance to communication, comprehensibility... (Helen: Yes)

Interviewer: And how do you feel about this? What is it like for you when it turns into a different kind of English?

Mona: Well, personally, ehm, - actually the same is true for German - well, I don't like it that much, but that's because I study English and I want to become an English teacher. But of course, at school, if they are speaking and if you can understand them, I think it's a good thing that they can at least communicate, even if they are saying "he don't", cause here I actually say to myself: So what? Many people are saying "he don't', English speaking people as well (laughs), so it's really OK for them to say that [...] (FG MA, 12.03.2012; 11:24)

Mona's attitude towards ELF is strongly dependent on the different identity options she mentions here. As an Erasmus student, she found it legitimate and useful to use Spanish as a lingua franca, even though many mistakes occurred and people used the language in unconventional ways according to their own linguistic background. Her present professional self as student teacher of 
English, however, cannot approve of such an emergence of "a different kind of English". Finally, in her imagined future role as teacher, she uses a different yardstick again, arguing that communicative competence should be the major goal for learning and that students should not be punished for mistakes that are even made by native speakers of English.

Data collected in İstanbul generated identity-sensitive discussions largely grounded in the native/non-native dichotomy. Braine (2010: 81) argues that a precise definition of proficiency is needed to gain clear insight to the issue of native speakerism. However, the focus group interviews conducted with postgraduate participants, some of whom hold positions as lecturers or even as administrators in tertiary education, revealed that the meaning of 'proficiency' kept on changing for the interviewees depending on the identities they foregrounded in that particular moment of interaction. In one participant's view, proficiency was initially "ease in self-expression" and "confidence in joining interaction" (FG $\mathrm{PhD} / 1,20.12 .2011,11: 25)$ but it soon turned out to be "comprehensible written production with relatively few errors" and "fluency, variety in word choice, correct intonation, use of phrasal, idioms and even informal, slang expressions to create humour" (FG PhD/1, 20.12.2011, 11:27) as the topic gradually shifted from learner to teacher proficiency in English. Despite his adherence to native-speaker norms in defining his vision of an ideal English language teacher, his 'small story', conveying his deeply felt frustration on receiving complaints from his freshman students about his status as a nonnative speaker of English, helped him "back-up or elaborate on [his] argumentative point" (De Fina/Georgakopoulou 2012: 116) that professional identity formation is largely hampered by the socially and institutionally fuelled 'native speaker idealism'. His case triggered further supportive remarks from other participants who reported many instances of institutional decisions on having native speakers teach intermediate and advanced English classes; as they claim, 'fluency' defined through native speaker norms does not only restrain non-native teachers from practicing their profession at different levels, but also disadvantages them in having further responsibilities in materials development and/or testing and evaluation departments.

Focus-group interviews conducted with student teachers also portrayed tensions and restraints arising from the privileging of native speaking English teachers in many institutions. As one interviewee recalls, all of his attempts to reflect and share pedagogical concerns on the model lessons he offered in a school were constantly interrupted by the mentor who insisted on correcting his speech. This, along with many other examples, shows that student teachers have the tendency to ground their experiences on a 'native and non-native dichotomy' rather than question the quality of mentoring. 


\subsection{Future perspectives with regard to ELF}

Seidlhofer (2011: 15) claims that the changing realities of English should influence the teaching profession to reframe its role "not by thinking the what, but rather the how, the latter being relatively more manageable, more subject to local control, and providing some measure of continuity". However, the research conducted in two Expanding Circle settings revealed the complexity and uncertainty in envisioning ELF-sensitive methodologies in the absence of a clear conceptualization of what ELF stands for in the participants' professional lives.

The concerns of participants from Berlin were largely focused on the possible drawbacks involved in implementation. They imagine it to be very difficult and time-consuming to organize ELF learning environments, e. g. because "you need students or other people from non-English speaking countries" (FG MA, 12.03.2012; 11:45). They also argue that it is not desirable to foster ELF learning as this means teaching "faulty language" and "an English which is oversimplified and reduced to its very basics" (ibid.). The participants from İstanbul addressed macro-level constraints on a possible transition to "un-conventional" (e. g. FG $\mathrm{PhD} / 2,27.12 .2011,10: 46$ ) practices in (ELF) language teaching; as one participant puts it, "the days of 'would you like a cup of tea?' is over" and there is more tolerance for forms such as "informations", yet it is rather too early to "quit 'is' and teach 'you be nice, man"' (FG PhD/2, 27.12.2011, 10:46-10:53) when all the teaching materials and centralized language exams for placement, recruitment and promotion are designed to foster standard language learning.

Some of the participants in both research contexts were aware of the immediate and increasing relevance of ELF for their future students and of its potential benefits in fostering 'intercultural exchanges', but they did not go as far as developing practical approaches or visions for teaching from this when, for them, opportunities for acquaintances with varieties of English were still limited not only for teachers, but also for language learners. When talking about their desired future selves, student teachers in İstanbul voiced out their aspiration to achieve more communicative approaches to be adopted in language classes, while their peers in Berlin expressed their wish to continue with present communicative language teaching models and ideals. A common conclusion drawn from their discussions is the need for a standard as the core of their educational enterprise, with lots of exposure to meaningful and authentic discourse and occasional "colouring" of lessons with varieties of English. Participants in Berlin mainly promoted immersion as the key to successful language learning during ages of schooling, and hence supported all kinds of intercultural experiences to be gained via travel and exchange projects. While accepting the existence of increasing opportunities for transnational experiences through digital spaces and mobility, participants in İstanbul highlighted the importance of "Life-long Learning" which can help language learners pursue 
further interest in learning 'Englishes' after school and invest further on their individual resources for developing their communication skills depending on the rising needs.

\section{Conclusions and further considerations}

The research findings indicate a mainly 'norm-dependent' conceptualization of English with little envisioning for ELF-sensitive methodologies. Nevertheless, the findings also show that Expanding Circle contexts cannot readily be identified as a homogeneous unit. The participants in İstanbul displayed a critical stance to the macro politics of language education which cherishes Inner Circle English and privileges the 'native speaker ideal', but only a few envision a transformative change in pedagogical approaches, which would challenge institutionally legitimized teaching practices. The participants in Berlin were more concerned about the micro dynamic implications of shifting the focus to ELF methodologies and questioned the need for such a transition. Despite the finding that one context is marked with the 'absence' and the other with the 'abundance' of first hand ELF experiences, the participants' conceptualization of ELF was often contradictory and fragmented in both settings.

The subjects' reflective engagement with their own experiences through narrative re-constructions and small stories offered valuable insights to the complex relation between ELF and identity. The multiple identities of the participants as ELF users, language learners, graduate students and/or prospective/practicing teachers, will constantly be influenced by the social, historical, cultural and political contexts they are part of. They might be influenced by the ELF discourses on their academic studies and first-hand ELF encounters, but also praised by their friends, students, families and colleagues for 'native-like' accents. Thus, the identities they claim for themselves as individuals and as future professionals might not be in alignment with the identities assigned to them by their social communities and institutions. In this vein, fragmented and contradictory views of ELF should not necessarily be interpreted as lack of awareness, but as a proof of identity work taking place when becoming a professional.

Phillipson (2008: 250) draws attention to the potential dangers involved in an uncritical acceptance of ELF as a "culturally neutral medium that puts everyone on an equal footing". This research not only offered insight to the diversity of two Expanding Circles in terms of prospective teachers' attitudes and beliefs on ELF, but also provided both the participants and the researchers with a critical reflective framework for questioning the implications of ELF for future English language teaching and English language teacher education. As Sifakis (2007) discusses, the post-modern phase of English language education - which 
requires new concepts and methodologies to keep up with the new realms of English - calls for a consequent change of perspective in language teacher education; this "transformative perspective" to teacher education needs to be endowed with critical reflections on ELF discourse and "more extensive awareness of communication and attitudinal, cultural, policy-related, historyrelated and pedagogical concerns" (ibid.: 359). The results of this research project indicate that, in order to trigger or at least create space for such 'transformative experiences' concerning ELF, pre-service teacher education should:

- empower student teachers to focus on the self by creating opportunities for identity-sensitive reflection on their emotions, aspirations, and experiences with respect to their profession and to re-envision their roles and possible strengths as non-native English teachers.

- establish a collaborative culture of negotiating and co-constructing key concepts such as 'proficiency', 'teacher qualifications', 'communicative methodologies', or 'professionalism' through a critical lens.

- invest in bi- or plurilateral research projects (e.g. on digital platforms) in which student teachers from different Expanding Circle countries act as research partners sharing their local pedagogical concerns, exploring the possibilities of creating transformative learning experiences, and generating their own ELF corpora to see how the language can be strategically 'accommodated' and 'adjusted' to achieve intercultural communication.

\section{References}

Adamson, Bob (2012). International Comparative Studies in Teaching and Teacher Education. Teaching and Teacher Education 28/5: 641-648.

Alvesson, Mats / Sköldberg, Kay (2009). Reflexive Methodology: New Vistas for Qualitative Research. 2nd edition. London: SAGE.

Barkhuizen, Gary (2011). Narrative Knowledging in TESOL. Online: http://www.tesolmedia. com/docs/teq261888r.pdf (last retrieval [19.03.2013]). 1-25.

Berns, Margie (2005). Expanding on the Expanding Circle: Where do WE go from here? World Englishes 24/1: 85-93.

Braine, George (2010). Nonnative Speaker English Teachers: Research, Pedagogy, and Professional Growth. NY: Routledge.

De Fina, Anna / Georgakopoulou, Alexandra (2012). Analyzing Narrative: Discourse and Sociolinguistic Perspectives. Cambridge: Cambridge University Press.

Dörnyei, Zoltán (2007). Research Methods in Applied Linguistics. Oxford: Oxford University Press. 
Fontana, Andrea / Frey, James H. (2005). The Interview: from Neutral Stance to Political Involvement. In: Denzin, Norman K. / Lincoln, Yvonna S. (eds.): The Sage Handbook of Qualitative Research. 3rd edition. Thousand Oaks: Sage Publications. 695-727.

Freeman, Mark (2007). Life “on holiday”? In Defense of Big Stories. In: Bamberg, Michael G. W. (ed.): Narrative - State of the Art. Amsterdam: Benjamins. 155-164.

Kachru, Braj B. (ed.) (1992). The Other Tongue: English across Cultures. 2nd edition. Chicago: University of Illinois Press.

Mackey, Alison / Gass, Susan M. (2005). Second Language Research: Methodology and Design. Mahwah, NJ: Lawrence Erlbaum.

Norton, Bonny (2000). Identity and Language Learning: Gender, Ethnicity and Educational Change. Harlow, England: Longman/Pearson Education.

Phillipson, Robert (2008). Lingua Franca or Lingua Frankensteinia? English in European Integration and Globalization. World Englishes 27/2: 250-235.

Seidlhofer, Barbara (2011). Understanding English as a Lingua Franca. Oxford: Oxford University Press.

Sifakis, Nicos C. (2007). The Education of Teachers of English as a Lingua Franca: a Transformative Perspective. International Journal of Applied Linguistics 17/3: 355-375.

Strauss, Anselm / Corbin, Juliet (1990). Basics of Qualitative Research: Grounded Theory Procedures and Techniques. Newbury Park, CA: Sage.

Vásquez, Camilla (2011). TESOL, Teacher Identity, and the Need for "Small Story" Research. TESOL Quarterly 45/3: 535-545.

Yano, Yasukata (2009). English as an International Lingua Franca: from Societal to Individual. World Englishes 28/2: 246-255. 



\title{
Comment retracer les compétences interculturelles en interaction dans la formation en ligne des enseignants de langues? \\ Une réflexion méthodologique
}

\author{
Mark Bechtel / Maud Ciekanski (Dresden, Paris)
}

\begin{abstract}
Despite a growing interest in telecollaboration since the 2000 s, few studies actually address the question of the assessment of intercultural communicative competence (ICC) developed by students or of the identification of intercultural skills as a condition of the emergence of the role of cultural mediator at the heart of these training settings. From a methodological perspective, the article explores the possibilities and limitations of methods for analysing ICC occurrences from a corpus of written peer interaction developed throughout the action research project INFRAL (INterculturel France Allemagne en Ligne - Intercultural France Germany Online).
\end{abstract}

Trotz des wachsenden Interesses an internetbasierten Formen der Zusammenarbeit beim Fremdsprachenlernen seit den 2000er Jahren befassen sich relativ wenige Studien mit der Frage, wie die interkulturellen Kompetenzen (IKK) der Lernenden als Bedingung für die Übernahme der Rolle als „kulturelle Mittler“ evaluiert bzw. wie diese empirisch nachgezeichnet werden können. Unter einer forschungsmethodischen Perspektive befasst sich der vorliegende Artikel mit Möglichkeiten und Grenzen des Erfassens und Beschreibens von Momenten der Aktualisierung interkultureller Kompetenz anhand eines Korpus aus schriftlichen Interaktionsdaten, die im Rahmen des Aktionsforschungsprojekts INFRAL (Interculturel France Allemagne Online) entstanden sind.

\section{Introduction}

Les moyens de communication qu'offre Internet ont permis l'expansion des projets de télécollaboration mettant en contact des apprenants ou futurs enseignants de langues, résidant dans des pays différents, pour la promotion des compétences langagières et interculturelles. Ces nouvelles formes d'«hyper-mobilités » (Dervin 2007: 99) renouvellent à la fois les modalités de contact en langues et la nature des dispositifs d'immersion, en prolongeant le temps d'interaction en langue-cible, tout en évitant des voyages coûteux. Ainsi depuis une vingtaine d'années, l'intérêt pour les dispositifs de télécollaboration perdure, comme en atteste l'importante littérature du domaine (Cononelos/Oliva 1993; Furstenberg et al. 2001; Fischer 1998; Kern 1996; O’Dowd 2003; von der Emde et al. 2001) et la recherche en didactique des langues a permis de mieux définir la nature de la compétence interculturelle, donnant lieu à différentes modélisations mobilisables par les enseignants et les chercheurs (ex. Byram 1997; Caspari/Schinschke 2009; Rössler 2010). Qu'apprend-on dans ces dispositifs? O'Dowd/Ritter (2006) montrent que bien souvent les interactions qui y sont pro- 
voquées ne dépassent pas le simple échange d'information sur la culture-cible et que les apprenants ne parviennent pas toujours à exploiter le potentiel formatif des interactions. L'attitude des participants (Jáuregui/Bañados 2008), leur façon de collaborer (Audras/Chanier 2008), leur familiarité à l'utilisation des nouveaux médias (Hauck 2007), la qualité du scénario pédagogique et l'organisation des échanges entre participants, semblent jouer un rôle important pour la réussite de tels projets.

En outre, l'évaluation de la compétence interculturelle demeure souvent problématique sur les plans de la formation et de la recherche, car reposant bien souvent sur l'utilisation de référentiels, portfolios, journaux ou questionnaires visant le déclaratif des participants, a priori ou à posteriori des situations de contact vécues, et donc détaché du moment de la mise en œuvre des compétences questionnées. Or ce discours ne peut être qu'une reconstruction des situations vécues et leur dimension formative est difficilement conscientisée par les participants. Pour ces raisons, l'observation in vivo (Dervin 2007: 114) des échanges par les participants eux-mêmes à partir des traces fournies par les outils de la communication médiée par ordinateur (CMO) (conservation des écrits dans les blogues, enregistrements de conversation orale, etc.) apporteraient une possibilité intéressante d'analyser l'apprentissage interculturel en tant que processus.

L'objectif de cette étude à visée méthodologique est de contribuer à faire avancer la réflexion sur l'identification des traces de la compétence de communication interculturelle (CCI) au niveau empirique, afin d'ouvrir de nouvelles perspectives sur le plan de la formation, notamment afin de favoriser l'émergence du rôle de médiateur culturel. Pour ce faire, nous questionnerons les possibilités et les limites de retracer le développement de la CCI à partir de données verbales (issues d'interaction et de discours réflexifs d'étudiants) recueillies dans le cadre de la recherche-action INFRAL (INterculturel France Allemagne en Ligne) en 2008, impliquant une équipe franco-allemande d'enseignants et de chercheurs des universités de Brême et de Franche-Comté (Abendroth-Timmer et al. 2013). La formation a regroupé une vingtaine d'étudiants de master français et allemands, tous futurs enseignants de Français Langue Étrangère (FLE).

Après avoir présenté le dispositif de télécollaboration INFRAL, nous discuterons le modèle de la CCI de Byram (1997) dont les catégories ont servi dans de nombreuses études comme indicateurs pour l'analyse de la compétence interculturelle, au regard de la nature des composantes de la CCI et de leur observabilité, en particulier en situation d'interactions. La partie centrale de l'article consistera à analyser différents procédés permettant de retracer les manifestations de la CCI et d'en comprendre le développement, en comparant, 
pour chaque procédé d'analyse, les possibilités méthodologiques et leurs limites, à partir des données recueillies dans INFRAL.

\section{Le projet de recherche-action INFRAL: une socialisation interculturelle renouvelée}

La partie formation du projet de recherche-action INFRAL a réuni pendant dix semaines dix étudiants francophones, en master de didactique du FLE à l'Université de Franche-Comté (UFC) et neuf étudiants germanophones en master de didactique des langues romanes à l'Université de Brême (UB), tous se destinant à enseigner le FLE. L'objectif de la formation était à la fois interculturel et professionnel. Dans une approche socioconstructiviste, le scénario pédagogique proposait de travailler quatre tâches, au sein de petits groupes réunissant de façon équilibrée quatre à six apprenants de l'UB et de l'UFC (des quadrems):

- se présenter et commenter la présentation des autres membres du quadrem (étape 1),

- présenter et expliquer une expérience interculturelle personnelle et commenter celles des autres membres du quadrem (étape 2),

- échanger sur la place et la nature de l'évaluation dans les pratiques enseignantes en France et en Allemagne à partir de ses propres expériences d'apprenant et d'enseignant que complète la lecture d'articles en français et en allemand sur l'évaluation à l'école (étape 3),

- élaborer de manière collaborative une tâche pédagogique visant le développement de la compétence culturelle à réaliser en classe de FLE, en France ou en Allemagne (étape 4).

À la fin de la formation, les étudiants ont rédigé un court rapport réflexif leur permettant de revenir sur leur apprentissage et de commenter les événements qu'ils jugeaient importants pour le développement de leur CCI (étape 5).

La collaboration entre apprenants a eu lieu intégralement en ligne dans deux espaces de communication:

- un blogue ${ }^{1}$ multi-auteurs pour la communication écrite asynchrone. Sur le blogue de leur quadrem, les apprenants ont pu, autant qu'ils le souhaitaient, poster des messages et des commentaires. Ils pouvaient également lire et réagir aux messages des blogues des autres quadrems de la formation sous formes de commentaires.

\footnotetext{
${ }^{1}$ Blogger: www.blogger.com.
} 
- une plateforme audiographique synchrone pour la communication orale. Tous les quinze jours et durant quatre séances, les quadrems se sont rencontrés en ligne sur la plateforme audio-synchrone Centra ${ }^{2}$ afin d'échanger à l'oral ou à l'écrit à travers différentes modalités de communication ${ }^{3}$.

Chaque étape du scénario pédagogique s'est déroulée en cinq phases, proposant un processus discussionnel à plusieurs niveaux, articulant oral et écrit, travail collectif et individuel, entre apprenants institutionnels et enseignant (phases 1 et 5) ou entre partenaires du même quadrem (phases 2, 3 et 4):

- Phase 1: une mise en route en présentiel, au sein de chaque groupe institutionnel, permettant aux étudiants d'aborder le thème de l'étape (30 min.).

- Phase 2: les échanges entre membres d'un quadrem dans le blogue (pendant 6 jours).

- Phase 3: la rencontre sur la plateforme audio-graphique synchrone permettant aux membres d'un quadrem de construire une synthèse collective des différents échanges produits dans le blogue $(1 \mathrm{~h})$.

- Phase 4: La rédaction dans le blogue de synthèse personnelles à partir des échanges produits au cours de l'étape du scénario pédagogique, permettant aux apprenants de prendre recul, de conscientiser certaines pratiques, de verbaliser ou expliciter la façon dont les échanges ont fait échos en eux (pendant 6 jours).

- Phase 5: le bilan en présentiel, au sein du groupe institutionnel, afin de dénouer les difficultés rencontrées $(1 \mathrm{~h})$.

En résumé, la situation d'apprentissage proposée était conçue comme un ensemble de conditions et de circonstances susceptibles d'amener les apprenants à construire des connaissances à partir de leurs expériences interculturelles passées et présentes. Afin de préserver au mieux l'authenticité des échanges entre les partenaires des quadrems, il a été décidé de limiter au maximum la présence de l'enseignant-tuteur en ligne.

\footnotetext{
${ }^{2}$ Centra: http://www.centra.com/french/products.asp.

3 Clavardage, tableau blanc, système iconique pour le non-verbal, téléchargement de diaporamas.
} 


\section{Du modèle de la compétence de communication intercultu- relle à l'identification des observables}

\subsection{Qu'est-ce que la compétence de communication intercultu- relle?}

Nous définissons la compétence de communication interculturelle (CCI) comme la capacité cognitive et comportementale qui recouvre un ensemble complexe de composantes interalliées (les savoirs, aptitudes et attitudes) en termes de communication interculturelle d'un individu.

Comme pour toute compétence, nous distinguerons trois traits principaux. D'abord, la CCI se manifeste à travers des performances observables dans l'action. Il s'agit pour l'individu d'une capacité d'agir de façon appropriée aux spécificités d'un contexte interculturel. Etre compétent, c'est aussi être reconnu comme compétent, ce qui implique la mobilisation des savoirs dotés d'une légitimité sociale permettant de se comporter de façon appropriée aux yeux des membres du groupe cible. Il s'agit tout à la fois d'un construit individuellement et socialement élaboré comportant nécessairement une composante négociative qui implique pour les participants d'opérer à un certain nombre de choix qui transparaît dans un travail de négociation, d'accomodation en ce qui concerne la co-définition de la situation, leurs rôles et leurs identités respectifs (ce que Byram nomme la mise en œuvre d'une vision du monde commune). L'interculturel en tant que mise en œuvre d'une relation à soi et à l'autre particulière recouvre donc un processus de distanciation-régulation singulier. Enfin, la CCI est évolutive: le niveau et la nature de la compétence d'un individu change en fonction de sa participation aux activités sociales, des fonctions communicatives, des aptitudes selon les normes et les besoins situationnels et intentionnels qu'il est en mesure d'identifier.

Dans la littérature du domaine, le modèle le plus répandu de la CCI est certainement celui proposé par Michael Byram (2000: 9; 1997: 57ff.) qui distingue un ensemble de cinq savoirs recouvrant les savoirs à proprement parler, aptitudes et attitudes mobilisables en interaction interculturelle:

- Le Savoir être: « curiosity and openness, readiness to suspend disbelief about other cultures and belief about one's own »

- Les Savoirs: « knowledge of social groups and their products and practices in one's own and in one's interlocutor's country, and of the general processes of societal and individual interaction »

- Le Savoir comprendre: " ability to interpret a document or event from another culture, to explain it and relate it to documents from one's own » 
- Le Savoir apprendre/faire: « ability to acquire new knowledge of a culture and cultural practices and the ability to operate knowledge, attitudes and skills under the constraints of real-time communication and interaction "

- Le Savoir s'engager: " ability to evaluate critically and on the basis of explicit criteria perspectives, practices and products in one's own and other cultures and countries »

À la suite de cette définition complexe et générale de la CCI, Byram (1997: 4954) a cherché à décliner la CCI en termes d'objectifs d'enseignement-apprentissage et d'évaluation, chaque savoir correspondant à un ensemble de sous-compétences en nombre variable ( 5 pour le savoir être, 11 pour les savoirs, 3 pour le savoir comprendre, 7 pour le savoir apprendre/faire et 3 pour le savoir s'engager). Ainsi, la compétence « savoirs/knowledge » comporte une large palette de manifestations différentes allant par exemple de «historical and contemporary relationships between one's own and one's interlocutor's countries » à " processes of social interaction in one's interlocutor's country ». L'intérêt de ces souscatégories est de permettre une compréhension plus fine de la façon dont la CCI peut se réaliser en interaction. Toutefois, la nature de ces sous-compétences regroupe en fonction des savoirs mobilisés à la fois des capacités (to identify, to elicit, to use), des attitudes (willingness, readiness, interest) et des connaissances (knowledge) qui ne se manifestent pas de la même façon et dont la mobilisation dépend à la fois du contexte et de l'individu. Par conséquent, ce découpage fin n'a pas été établi à l'origine dans l'objectif de permettre l'analyse d'échanges réalisés entre interlocuteurs de cultures différentes, même si un certain nombre de chercheurs s'en sont servis afin d'identifier les manifestations d'une CCI.

\subsection{Quels observables pour la recherche?}

Si Byram (1997) lui-même ne s'intéresse pas directement à la façon dont la CCI peut être repérée en interaction, il insiste toutefois sur la nécessité d'inventer une méthode permettant de l'évaluer globalement (le choix d'une perspective holistique).

Pour Lu/Corbett (2010: 90), l'inventaire des savoirs proposé par Byram (1997) propose un cadre pertinent et utile pour déterminer les compétences attendues chez un médiateur culturel (en tant que modèle à atteindre). Ils notent toutefois que les compétences pragmatiques (qui se révèlent en action) ne peuvent pas être directement évidentes et plaident également pour une conception holistique de la formation à la CCI à travers l'action, la réflexion et la reproduction, ces prises de conscience interculturelles permettant ainsi un autre accès à la mise en œuvre de la CCI, notamment à travers le déclaratif des participants, ce qui n'est pas sans poser problème comme nous le verrons ultérieurement. Comment procéder? 
L'une des difficultés à identifier le CCI en termes d'observables provient du fait que ses manifestations dépendent grandement des situations créées et la façon dont les apprenants parviennent à y répondre. L'évaluation de la performance (Byram 1997: 105) permettrait de suivre la façon dont le savoir et les habiletés apparaissent en situation(s), mais elle peut évoluer selon les individus. Cela pose également la question de sa transférabilité à de nouvelles situations: ce que l'on sait faire dans un contexte peut ne pas être fait de façon appropriée dans un autre contexte.

Partant de ce constat, les chercheurs ont essayé d'identifier des situations ou des performances clefs capables de révéler la CCI. Il s'agit par exemple de s'intéresser à la nature des changements de perspectives (Bechtel 2009) ou à leur fréquence (Byram 1997: 108). D'autres chercheurs ont privilégié la capacité à résoudre un problème, un conflit ou une incompréhension. Toutefois, tout contexte de CCI ne fait pas forcément apparaître ce genre de situations sensibles (cf. Ciekanski, à paraître, où les objectifs de socialisation peuvent l'emporter sur des objectifs interculturels en tant que tels). La difficulté de la tâche de repérage va donc dépendre de la complexité des données à manipuler par les participants (input) et de la localisation et la fréquence des manifestations de la CCI (Byram 1997: 110).

En outre, comment répondre à la question des degrés de manifestation de la CCI? Byram (1997: 90) par exemple évoque la notion de « deep learning » et « shallow learning ». Il s'agirait alors de privilégier ce qui est le plus pertinent, le plus approprié à la situation de communication et de vérifier selon Byram la façon dont les savoirs appartiennent à l'apprentissage profond et permettent à l'apprenant de les manipuler, les utiliser à bon escient.

Néanmoins, puisque le CCI relève d'une 'agentivité', sa manifestation ne peut se limiter à la stricte performance. Ainsi, pour Byram (1997: 93), il est important de distinguer entre performance et attitude en invitant les participants à expliquer leurs choix: " it would also be possible to obtain 'evidence' from the same performance for learner's savoirs and their savoir comprendre ». Toutefois faire relever les indices de la CCI par les participants eux-mêmes requiert un fort degré de conscience de soi et d'habilité à réfléchir sur ses propres actions (cf. Ciekanski, à paraître). Enfin, comme nous l'avons rappelé, la CCI dépend du contexte social dans lequel elle prend place or les dimensions de la communication non-verbale sont importantes: la question des non-dits et le modèle de l'iceberg de la CI illustrent bien les limites de la focalisation sur la 'dimension langagière' de la CCI. Le jeu des perceptions mutuelles des identités sociales des participants est tout aussi déterminant dans le déroulé des interactions (la capacité à négocier explicitement ou implicitement son rôle, ses actes, ses dires, la perception de la différence de l'autre dans l'ajustement des phénomènes de normes et de convention de socialisation, etc.). 


\section{Retracer les compétences interculturelles en interaction dans la formation en ligne des enseignants de langues: potentiels et limites}

Le recueil des données recourt à une méthode in vivo ${ }^{4}$ pour les transcriptions des interactions écrites (blogues) et les enregistrements des interactions orales (audio-conférence), complétée par une méthode qualifiée d'in vitro (proactive ou réactive mais détachée du contexte de mise en œuvre des compétences) pour les pré- et post-questionnaires et le rapport réflexif individuel rédigé par les étudiants à l'issue de la formation leur permettant de revenir sur les moments perçus comme marquants (positifs ou négatifs) dans l'expérience proposée. Nous nous concentrons pour cette étude sur les interactions écrites dans le blogue du quadrem 4 (il s'agit du blogue le plus prolixe) ainsi que sur les rapports réflexifs de ses membres.

A partir de nos premières analyses réalisées sur le corpus INFRAL, nous proposons une lecture critique de la méthodologie utilisée dans le champ de la didactique des langues pour tracer le développement de la CCI, en mettant en regard quatre procédés différents de façon à en souligner la pertinence pour l'analyse des données verbales issues pour partie d'interactions en ligne. Pour chaque procédé, nous montrerons son fonctionnement, son potentiel et ses limites illustrés par des exemples issus de notre corpus.

\subsection{Analyse pour l'exemplification des grandes catégories du modèle de Byram}

Une des possibilités d'identifier la CCI chez les participants est pour le chercheur de reprendre les grandes catégories du modèle de Byram, et de rechercher dans le corpus pour chacune (ou pour un certain nombre de ces catégories) des passages d'échanges montrant, selon lui, que le participant a manifesté une certaine facette de la CCI. Il s'agit d'une observation à posteriori transformant les objectifs de formation et d'évaluation du modèle de Byram en indicateurs de la manifestation d'une ou plusieurs facettes de chaque savoir composant la CCI.

\subsubsection{Fonctionnement}

Pour attester de la catégorie 'attitudes' (le 'savoir-être'), c'est-à-dire d'une ouverture de soi et une curiosité envers l'autre, nous pourrions citer un certain nombre de phrases des quatre participants du quadrem 4 dans lesquelles les

\footnotetext{
${ }^{4}$ Nous reprenons à notre compte la distinction proposée par Dervin (2007: 114) pour qualifier le travail des compétences interculturelles en contexte ou hors contexte.
} 
étudiants expriment leur intérêt à connaître les autres et à travailler avec eux («Bienvenue dans Infral08! Je m'appelle ambre4_15 et je me rejouis déjà beaucoup de travailler avec vous et de lire vos petits textes. Bonne journée et à bientôt! », ambre4_1), à parler d'eux-mêmes («Si vous avez encore des questions concernant ma personne, n'hésitez pas à me demander », afbre4_2) ou leur envie de profiter de ce groupe pour apprendre mutuellement les uns des autres ( Nous avons la chance dans notre groupe d'avoir 3 nationalités différentes alors profitons de cette diversité! », afbes4_1).

Pour témoigner de la catégorie 'discover and interact' (le 'savoir apprendre/faire') nous pourrions entre autre citer un passage d'un message dans le blogue de afbes4_1 (voir annexe 1). La manifestation de cette compétence se trouve dans le fait qu'afbes4_1, après avoir essayé de comprendre les explications de son interlocuteur, (1) reprend ce qu'elle a compris (« tu dis qu'il n'y a que... »), (2) formule une affirmation sur l'autre culture en la transformant en supposition par un point d'interrogation («alors il n'y a pas d'évaluation qui sanctionne le passage de la 1ere à la 2ème année et de la 2ème année à la 3 année ?») et (3) met en relief pour l'interlocuteur son système de référence à elle (« en France, à l'école primaire il y a des 'contrôles' pour chaque année»).

\subsubsection{Potentiel}

Le potentiel de ce procédé est de pouvoir déterminer si les interlocuteurs ont manifesté, dans leurs interactions ou dans leur rapport réflexif, certaines compétences interculturelles et la nature de ces dernières. Plusieurs chercheurs ont recours à ce procédé d'analyse. Dans un projet de télécollaboration entre deux universités, l'une aux Etats-Unis (Pensylvania), l'autre en Allemagne (Gießen, puis Heidelberg), Müller-Hartmann (2005), en se basant sur les rapports des étudiants allemands, prend trois catégories du modèle de Byram une par une et résume les manifestations de la CCI en donnant des exemples issus des données verbales, sans pour autant préciser les indicateurs linguistiques retenus. Audras/ Chanier (2008) se sont concentrés dans leur projet sur deux catégories ('le savoir être' et 'le savoir-connaissance') du modèle de Byram. Le relevé des compétences observées se fonde sur le repérage d'éléments verbaux, parmi les productions d'apprenants, permettant d'établir une correspondance avec les grandes catégories de Byram, en prenant en compte le tour de parole et le contexte conversationnel. Ce sont ensuite les éléments lexicaux (entre autre la

${ }^{5}$ Le prénom des participants a été annonymisé: $\mathrm{a}=$ "apprenant", $\mathrm{m}=$ masculine, $\mathrm{f}=$ féminin, bes $=$ Besançon, brem $=$ Bremen, le premier chiffre indique le quadrem (ici quadrem 4), le deuxième permet d'identifier les membres du quadrem. 
distribution tu/vous dans le système d'adressage) qui sont repérés comme porteurs d'information sur l'existence de telle composante du modèle de la CCI.

\subsubsection{Limites}

Ce type de procédé pose plusieurs problèmes. Premièrement, pour que l'attribution d'un énoncé à une catégorie du modèle de Byram soit valide, il faudrait une plus grande concertation entre chercheurs ce qui est rarement le cas, dans la mesure où la plupart des travaux font état d'un repérage individuel (ex. Audras/Chanier 2008). Deuxièmement, on peut avancer que l'établissement de compétences en unité sécable les unes des autres est 'artificiel' au détriment de l'interrelation de plusieurs facteurs dans la CCI (Byram parle d'ailleurs de facteurs en interaction les uns les autres). Troisièmement, même si le chercheur justifie l'étiquetage des énoncés en citant comme référence un passage du corpus, l'extrait relevé appartient souvent à un contexte plus large d'échanges et il nous a semblé parfois que, en séparant l'extrait de son contexte, la CCI relevée perdait de son évidence. La question qui se pose est donc celle de la taille du grain d'analyse: faut-il mettre des étiquettes par messages ou bien par parties de messages? Quatrièmement, ce procédé qui se limite à des exemples illustrant la manifestation d'une grande catégorie de la CCI de Byram dans le corpus ne permet pas d'analyser comment les différentes catégories sont réparties par participant et par le temps. Ce procédé ne répond donc pas ici à la question de l'émergence ou du développement de la CCI. Enfin, on peut s'interroger sur le fait que la CCI que le chercheur a essayé de retracer ainsi est le résultat de la formation proposée ou si elle a été acquise auparavant par les participants. Byram (1997: 49) lui-même a souligné cette problématique:

The objectives designate a range of skills, knowledge and attitudes which may not necessarily be the outcome of learning directly related to language learning, since they may include phenomena already present in the learner before language learning begins.

Enfin, la question du relevé des manifestations à un instant donné questionne le caractère 'acquis' de la $\mathrm{CCI}$, dans la mesure où comme toute compétence elle se définit avant tout comme développementale et situationnelle - ce qui n'apparaît pas aujourd'hui peut se manifester demain, ce qui apparaît dans une situation donnée ne peut se révéler dans une autre situation.

\subsection{Analyse quantitative à l'aide des sous-catégories du modèle de Byram}

Un autre procédé d'analyse concerne le codage systématique des énoncés de chaque participant du début jusqu'à la fin de l'échange, à l'aide des sous-catégories du modèle de Byram, et de les compter. 


\subsubsection{Fonctionnement}

Le chercheur prend en compte systématiquement tous les énoncés et leur attribution à une des grandes catégories du modèle de Byram en utilisant les souscatégories de ce modèle. En transformant le résultat de cette analyse en un tableau quantitatif, on obtient une vue d'ensemble des manifestations de la CCI, en mettant en relief les catégories les plus ou moins présentes dans les échanges.

\subsubsection{Potentiel}

Le potentiel de ce procédé est que le codage nécessite une analyse systématique de l'ensemble des interactions, donc va au-delà de l'exemplification. Le procédé permet de dégager des grandes tendances dans la fréquence des manifestations de la CCI et leur répartition, de faire des comparaisons entre les participants par étape ou sur l'ensemble de l'échange ou de relier les manifestations de la CCI aux tâches du scénario pédagogique. Il permet de travailler à plusieurs sur un corpus donné, force les chercheurs à se mettre d'accord sur l'étiquetage effectué et sur l'évidence des indicateurs pour les différentes catégories.

\subsubsection{Limites}

L'analyse quantitative nécessite le comptage de l'occurrence des items pour chaque catégorie. Le problème est donc celui du double codage dans la mesure où les énoncés sont souvent la manifestation de deux ou trois catégories à la fois, puisqu'il s'agit d'une compétence complexe qui combine connaissances, aptitudes et attitudes. Cette évidence pose donc problème lorsqu'il s'agit de compter leur occurrence. Un autre problème est que les sous-catégories ne sont pas strictement séparées, elles se chevauchent, ce qui rend parfois le codage difficile. Pour nos premières analyses chacun avait procédé individuellement à son propre étiquetage pour le blogue du quadrem 4. Nous avons constaté que le nombre des messages et commentaires rélevé comme présentant une ou plusieurs compétence(s) interculturelle(s) différait. Sur les messages et commentaires relevés, très peu ont été étiqueté de façon identique à l'aide des souscatégories de Byram. Le problème provient à la fois de l'interprétation des catégories de Byram et la façon dont on les reconnait dans les données verbales du corpus, étant donné qu'une grande partie des indices peut être implicite ou sous-entendue. Un autre inconvénient de ce procédé est que la présentation du résultat de l'étiquetage dans un tableau quantitatif ne permet plus de vérifier la plausibilité du codage, le grand nombre de messages/commentaires pris en compte ne permettant plus de référer aux énoncés choisis. Enfin, une représentation quantitative de l'occurrence des manifestations de la CCI n'informe en rien sur le processus d'apprentissage, car on ne voit plus quel énoncé a provoqué 
quelle réaction chez les interlocuteurs, puisque le déroulement des interactions n'est plus accessible.

\subsection{Analyse séquentielle des données verbales choisies par le chercheur}

Au lieu de regrouper les énoncés des participants par catégories, des séquences d'interaction entre les participants peuvent être reconstituées dans les données verbales lorsqu'elles sont identifiées par le chercheur comme manifestant un moment d'apprentissage interculturel.

\subsubsection{Fonctionnement}

Afin de pouvoir reconstruire le déroulement de l'interaction dans le blogue, il est nécessaire de disposer de toutes les informations sur la nature (message, commentaire), le destinataire, la date et l'heure de l'énoncé. Les pages html des blogues fournissent ces informations. Un exemple de notre corpus illustre ce procédé (cf. annexe 2): Il s'agit d'une séquence dans l'étape 4 où les membres du quadrem 4 avaient proposé des idées pour une tâche interculturelle et sont en train de demander et de donner des précisions entre eux. Lors de la séquence, afbes4_1 avait demandé des précisions par rapport à une description de afbre4_2 que cette dernière fournit. Il s'agit ici d'une comparaison entre la France et l'Allemagne par rapport à la date de la remise des cadeaux de Noël. Afbre4_2 montre la compétence de mettre en relation deux cultures (le 'savoir comprendre') qui comporte à la fois un savoir-connaissance sur sa propre culture qu'elle communique pour les autres ( $«$ le $24 / 12$ pour la remise des cadeaux ») et un savoir-connaissance sur la culture étrangère, ainsi qu'un 'savoir apprendre'. Comme afbre4_2 n'est pas sûre de son savoir sur la culture étrangère elle utilise la stratégie de montrer que ce savoir est une supposition qu'elle aimerait faire vérifier par un natif, en l'occurrence par afbes4_1, en lui demandant si sa supposition est valide ("C'est le soir du 24 et l'élève allemand qui fête Noël en France attend des cadeaux, mais il n'y en a pas parce que les cadeaux c'est le 25. C'est vrai, non?»). La réponse du natif vérifie la supposition de afbre4_2, ce qui nous amène à supposer que cela contribue à confirmer le savoir du non-natif. En même temps, le natif donne une précision sur ce phénomène culturel quant au moment de la remise des cadeaux en faisant référence à une perspective individuelle ( en ce qui me concerne, j'ai toujours reçu mes cadeaux le matin du 25 décembre! ») tout en ouvrant la perspective à une diversité intra-culturelle ("Mais je connais des gens qui reçoivent leurs cadeaux le 24 au soir! [Ç]a dépend des familles! »). 


\subsubsection{Potentiel}

Le potentiel de ce procédé de nature qualitative est d'identifier les moments d'un apprentissage interculturel dans l'interaction en considérant chaque 'tour' comme un élément potentiel de cet apprentissage. Le chercheur peut ainsi essayer de reconstruire les stratégies employées par les participants à apprendre au niveau culturel et à aider l'interlocuteur à mieux comprendre. L'avantage de ce procédé est que les énoncés ne sont pas décontextualisés. Ils sont analysés dans le contexte de tâche pédagogique, messages et commentaires permettant à reconstruire les fils de discours et tracer le processus de co-construction propre à ce type de situation d'apprentissage par échanges. Le chercheur peut également discuter à l'aide des séquences de ce qui est laissé de côté et de ce qui n'apparaît pas au niveau des compétences interculturelles.

\subsubsection{Limites}

Comme la reconstruction séquentielle d'une interaction d'un seul message avec ses commentaires reliés demande beaucoup de place pour la description et l'analyse, ce procédé implique forcément de se focaliser sur un choix de séquences en laissant de côté de nombreuses autres, le risque étant de rester à un niveau d'exemplification et non de compréhension globale. Un autre problème est que le chercheur identifie des séquences qu'il croit être significatives pour la manifestation d'une compétence de la CCI ou d'un apprentissage interculturel chez les apprenants. Mais les apprenants auraient-ils choisi les mêmes séquences pour montrer à quels moments ils ont appris quelque chose? Le problème serait que le chercheur accorde de l'importance à certains passages qui, aux yeux de l'apprenant, n'ont pas eu de signification dans son apprentissage.

\subsection{Analyse séquentielle des données verbales choisies par les participants}

Dans ce procédé le chercheur prend en compte exclusivement les moments de l'interaction dans les blogues qui, dans les rapports réflexifs, ont été mentionnés par les participants comme moments significatifs pour l'apprentissage interculturel. Il s'agit d'événements qui ont déclenché une conscientisation ou réflexion chez l'apprenant et qui peuvent - ou non - donner lieu à un apprentissage (certains moments ou événements négatifs peuvent ainsi empêcher la mise en œuvre de certaines actions relevant de la CCI). Les participants prennent ici la posture de l'auto-ethnographe, devenant expert dans l'explicitation des situations qu'ils ont vécues. 


\subsubsection{Fonctionnement}

Le point de départ de ce procédé est le rapport réflexif d'un participant, nous prenons ici à titre d'exemple ambre4_1 (cf. annexe 3). Le participant mentionne un moment dans le travail en quadrem qui a été pour lui particulièrement important. Il s'agit d'un moment dans le blogue où il a profité de la présence d'un natif pour lui poser une question sur un phénomène culturel perçu en France lors d'un séjour, question à laquelle il a toujours voulu obtenir une réponse. Le chercheur essaie de retrouver ce moment dans la transcription des blogues, il s'en suit une analyse séquentielle comme pour le procédé précédent. L'analyse séquentielle montre que ambre4_1 manifeste ici un 'savoir-apprendre' car il prend l'initiative de décrire un phénomène de la culture étrangère, puis demande le point de vue d'un natif afin d'avoir une perspective d'un insider que celui-ci lui fournit.

\subsubsection{Potentiel}

Le croisement entre le rapport réflexif et les interactions dans le blogue permet de focaliser l'exploration des blogues sur les moments que les participants euxmêmes identifient comme significatifs pour leur apprentissage interculturel. Ainsi, le chercheur réduit le risque de sur-interpréter l'importance pour l'apprentissage interculturel des passages que seulement lui-même avait repérés comme intéressants sans que cela soit partagé par les participants eux-mêmes.

Lire les échanges à travers les yeux des participants permet en outre d'éclairer la dimension informative et formative des échanges. Les rapports réflexifs apportent un complément d'information qui n'apparaît pas dans le blogue et donc compense la part d'implicite - notamment dans la façon dont les échanges sont vécus - des données verbales.

\subsubsection{Limites}

Lorsque le rapport réflexif est le point de départ de l'identification des moments importants pour l'apprentissage aux yeux des participants, la consigne pour la rédaction du rapport réflexif doit être formulée de façon à focaliser sur cet aspect. Le problème avec la focalisation sur des 'moments critiques' est qu'elle ne garantit pourtant pas l'élicitation de ce qui était vraiment important pour les participants. Byram, qui propose lui de travailler à partir d'une « autobiographie d'expériences interculturelles clés ", offre lui-même une critique très juste de cette méthode (Byram 2005: 14):

It is problem focused, it only deals with experiences which reflect difference and there may be a tendency to focus on difficulties rather than pleasurable experiences, but 'key experiences' are not necessarily difficult or problematic. 
Même si les rapports réflexifs recueillis ne sont pas majoritairement focalisés sur des aspects problématiques, sans focalisation précise, ils restent, en règle générale, vagues et peu explicites. C'est ainsi que souvent certaines informations données ne permettent pas directement d'être reliées à des éléments discursifs dans les blogues. Une limite de ce procédé est qu'il dépend beaucoup de la capacité méta-réflexive et méta-cognitive des participants. Arrivent-ils à mettre en mots ce qu'ils ont appris et comment? Byram (2005: 14) mentionne également ce problème: "The discursive account requires a high degree of literacy and analytical skill », que de nombreux apprenants utilisateurs n'ont pas à leurs dispositions (Ruben 1989: 235). En outre, demander à un apprenant utilisateur de tenir des journaux de bord ou de rédiger un rapport réflexif à la fin de l'expérience laisse supposer que celui-ci fait preuve de franchise et de conscience de soi, alors que les compétences interculturelles sont souvent construites à partir d'apprentissages buissonniers que l'apprenant utilisateur ne souhaite pas toujours partager (cf. Ciekanski à paraître).

Un procédé combinant les deux derniers procédés est celui du 'stimulated recall'. Le chercheur identifie les moments importants ou les participants identifient eux-mêmes les éléments importants au cours de leur activité, puis les participants sont amenés à expliciter leur processus d'apprentissage. Il s'agit d'une approche contextuelle qui pourra contribuer à relativiser l'interprétation du chercheur et aider le participant à mettre en mots son expérience.

\section{Conclusion}

La lecture critique des quatre procédés d'analyse de données verbales pour retracer les compétences interculturelles a montré que chaque procédé a un certain potentiel mais en même temps des limites. La triangulation des procédés semble être un bon moyen de profiter du potentiel de chaque procédé.

Tous les procédés exposés sont confrontés à un grand défi, celui du repérage des compétences interculturelles dans des données verbales. A quel point les catégories larges du modèle de Byram aident-elles à distinguer entre les différentes composantes de la CCI pour le travail d'analyse? A quel point les souscatégories du même modèle le permettent-elles? Quels sont les indicateurs (pragma-)linguistiques et socio-pragmatiques qui aident à ce repérage? Comment peut-on limiter la subjectivité du codage par les chercheurs?

A partir de ce problème méthodologique il nous semble important de retenir que les chercheurs devront se rappeler que les compétences interculturelles ne sont pas acquises à vie, et qu'un nombre illimité de facteurs interviennent dans leur application (fatigue, sympathie-antipathie, lieux de rencontre, etc.). Nous sommes d'accord avec Zarate (2003: 113; Dervin 2010: 108), qui insiste pour parler de compétences interculturelles au pluriel, car celles-ci peuvent être 
déclinées en plusieurs phases de développement non fixées. En effet, les compétences sont instables dans la mesure où elles sont basées sur du cognitif, mais également sur de l'affectif et de l'interactif; ainsi, comme nous le soulignions plus haut: une personne qui est normalement " compétente » peut s'avérer « incompétente » dans d'autres contextes.

\section{Bibliographie}

Abendroth-Timmer, Dagmar / Bechtel, Mark / Chanier, Thierry / Ciekanski, Maud (2013). Grenzüberschreitendes Blended-Learning. Zur Förderung interkultureller Kompetenz in der universitären Französischlehrerausbildung. In: Rössler, Andrea (éd.): Standards interkultureller Kompetenz für Fremdsprachenlehrer. Landau: Verlag Empirische Pädagogik. 165-182.

Audras, Isabelle / Chanier, Thierry (2008). Observation de la construction d'une compétence interculturelle dans des groupes exolingues en ligne. Alsic 11/1 (document alsic_v11_17rec10) Online: http://alsic.revues.org/865 (dernier accès [25.07.2013])

Bechtel, Mark (2009). Empirische Untersuchung zu interkulturellem Lernen in deutsch-französischen Tandemkursen mit Hilfe der Diskursanalyse. In: Hu, Adelheid / Byram, Michael (éds.): Interkulturelle Kompetenz und fremdsprachliches Lernen. Modelle, Empirie, Evaluation. Tübingen: Narr. 139-158.

Byram, Michael (1997). Teaching and Assessing Intercultural Communicative Competence. Clevedon: Multilingual Matters.

Byram, Michael (2000). Assessing Intercultural Competence in Language Teaching. Sprogforum 18/6: 8-13. Online: http://inet.dpb.dpu.dk/infodok/sprogforum/Espr18/byram.html (dernier accès [25.07.2013])

Byram, Michael (2005). European Language Portfolio. Theoretical model and proposed template for an autobioraphy of 'key intercultural experiences'. Strasbourg, Language Policy Division: Council of Europe.

Caspari, Daniela / Schinschke, Andrea (2009). Aufgaben zur Feststellung und Überprüfung interkultureller Kompetenz im Fremdsprachenunterricht - Entwurf einer Typologie. In: $\mathrm{Hu}$, Adelheid / Byram, Michael (éds.): Interkulturelle Kompetenz und fremdsprachliches Lernen. Tübingen: Narr. 273-285.

Ciekanski, Maud (à paraître). Développer la compétence (inter)culturelle des enseignants de FLE en ligne par le biais de l'apprentissage biographique. Paris: Editions Riveneuve, coll. Actes Académiques.

Cononelos, Terri / Oliva, Maurizio (1993). Using Computer Networks to Enhance Foreign Language/Culture Education. Foreign Language Annals 26/4: 527-534.

Dervin, Fred (2007). Evaluer l'interculturel: problématiques et pistes de travail. In: Dervin, Fred / Suomela-Salmi, Eija (éds.): Evaluer les compétences langagières et interculturelles dans l'enseignement supérieur. Turku: Publications du département d'études françaises. 95-122.

Dervin, Fred (2010). Assessing intercultural competence in Language Learning and Teaching: a critical review of current efforts. In: Dervin, Fred / Suomela-Salmi, Eija (éds.): New 
Approaches to Assessing Language and (Inter-)Cultural Competences in Higher Education. Frankfurt/M.: Lang. 157-173.

Fischer, Gerhard (1998). E-mail in Foreign Language Teaching: Towards the Creation of Virtual Classrooms. Tübingen: Stauffenburg Medien.

Furstenberg, George / Levet, Sarah / English, Kathryn / Maillet, Katherine (2001). Giving a voice to the silent language of culture: The Cultura Project. Language Learning \& Technology 5/1: 55-102. Online: http://ltt.msu.edu/vol5num1/furstenberg/default.html. (dernier accès [25.07.2013])

Hauck, Mirjam (2007). Critical success factors in a TRIDEM exchange. ReCALL Journal 19/2: 202-223.

Jáuregui, Kristi / Bañados, Emerita (2008). Virtual interaction through video-web communication: A step towards enriching and internationalizing language learning programs. ReCALL Journal 20/2: 183-207.

Kern, Richard (1996). Computer-mediated communication: Using e-mail exchanges to explore personal histories in two cultures. In: Warschauer, Mark (éd.): Telecollaboration in Foreign Language Learning. University of Hawai'i: Second Language Teaching and Curriculum Center. 105-109.

Lu, Peih-ying / Corbett, John (2010). The Health Care Professional as Intercultural Speaker. In: Levine, Glenn S. / Phipps, Alison (éds): Critical and Intercultural Theory and Language Pedagogy. Florence, KY, USA: Cengage Learning. 76-94.

Müller-Hartmann, Andreas (2005). Interkulturelles Lernen in internationalen Telekollaborationsprojekten. Die Entwicklung von Erfahrungswissen angehender Fremdsprachenlehrer/ innen. Fremdsprachen Lehren und Lernen 34: 192-206.

O’Dowd, Robert (2003). Understanding the "other side": Intercultural learning in a SpanishEnglish-e-mail exchange. Language Learning \& Technology 7/2: 118-144. Online http://lt.msu.edu/vol7num2/odowd/default.html. (dernier accès [25.07.2013])

O’Dowd, Robert / Ritter, Markus (2006). Understanding and Working with ,Failed Communication' in Telecollaborative Exchanges. CALICO Journal 23/3: 623-642.

Rössler, Andrea (2010). Standards für interkulturelle Kompetenz im Spanischunterricht. Hispanorama 127: 12-19.

Ruben, Brent D. (1989). The study of cross-cultural competence: Traditions and contemporary issues. In: International Journal of Intercultural Relations 13: 229-240.

von der Emde, Silke / Schneider, Jeffrey / Kötter, Markus (2001). Technically Speaking: Transforming Language Learning through Virtual Learning Environment (MOOs). The Modern Language Journal 85/11: 210-225.

Zarate, Geneviève (2003). Identities and plurilingualism: preconditions for the recognition of intercultural competences. In: Byram, Michael (ed.): Intercultural Competence. Strasbourg: Council of Europe. Online: http://theewc.org/uploads/content/Intercultural Competence_EN.pdf [dernier accès (14.11.2013)] 


\section{Annexe 1}

Dạkke schön ambre4_1 ! Je te remercie d'avoir pris le temps de répondre à mon long messsage et tes explications sont claires et précises!

Cependant j'aimerais te poser d'autres petites questions( mais plus par rapport aux mots composés!lol! C'est la folie en allemand ces mots à rallonge!lol!Un long mot en Allemand correspond à une phrase en Français!!!!).

Bon alors mes questions:

Tu dis qu'il n'y a que des notes à partir de la troisième année mais vu que die Grundschule dure 4 ans alors il n'y a pas d'évaluation qui sanctionne le passage de la 1ere à la 2 ème année et de la 2ème année à la 3ème année?

En france, à l'école primaire il y a des 'contrôles' pour chaque année.

Je me suis renseignée sur les différents examens pour les différentes filières (Realschule, Hauptschule, Gymnasium) et donc j'ai appris que le 'Abitur' c'est seulement pour le 'lycée', c'est ça? Et pour certaines filières il faut obtenir de bonnes notes (par ex en médecine) mais pour d'autres peu importe, il suffit la moyenne?

Dernière question: en France, la moyenne c'est 10 (si on est noté sur 20), pour vous c'est

'Ausreichend'? ou entre 'Befriedigend' et 'Ausreichend'?

J'ai deviné ta note en Français: Sehrgut: die beste Note (stimmtdas?)

Bis bald!!!

\section{Annexe2}

(comment, tag:blogger.com,1999:blog-5539687492853290518.post-4453376326231905700)

\# in_reply_to(tag:blogger.com,1999:blog-5539687492853290518.post-6765937155074308720)

\# author(afbre4_2)

\# published(2008-12-07, 13:12:00.000, +01:00)

\# updated(2008-12-07, 13:12:28.193, +01:00)

\# content :

1 Bon. Je vais essayer de préciser.

2 J'ai pensé que les élèves peuvent inventer une scène dans laquelle un élève fête Noel dans une autre culture. L'élève voit comment ça se passe, mais il ne comprend pas se qui se passe.

3 Pour donner un exemple :

$4 \quad$ C'est le soir du 24 et l'élève allemand qui fête Noël en France attend des cadeaux, mais il n'y en a pas parce que les cadeaux c'est le 25. (C'est vrai, non ?)Donc il est dans cette famille et il se demande ce qui se passe.

5 Donc un groupe joue cette scène sans parler. Que celui qui est l'élève de l'autre culture parle. Il dit comment il se sent, ce qu'il voit. Pourquoi il ne comprend pas ce qui se passe.

6 Les autres regardent la scène puis ils discutent ce qu'ils ont vu. Comme cela ils apprennent qu'il y a des différences entre des culture, mais que ce n'est pas grave. Qu'il faut plutôt faire l'effort de comprendre l'autre...

$7 \quad$ J'espère que j'ai pu éclaircir ma proposition. Il y a encore d'autres questions?

\# entry(comment, tag:blogger.com,1999:blog-5539687492853290518.post-4427391316668143290)

\# in_reply_to(tag:blogger.com,1999:blog-5539687492853290518.post-6765937155074308720)

\# author(afbes4_1) \# entry

\# published(2008-12-07, 14:56:00.000, +01:00)

\# updated(2008-12-07, 14:56:05.245, +01:00)

\# content:

$1 \quad$ Salut acd(afbre4_2)!

2 Oui c'est bon j'ai compris, merci! de tout de manière on a la séance centra pour en discuter! 
3 Petite précision: en ce qui me concerne, j'ai toujours reçu mes cadeaux le matin du 25 décembre! Mais je connais des gens qui reçoivent leurs cadeaux le 24 au soir! ça dépend des fa milles! bye!

\section{Annexe 3: Extrait du rapport réflexif de ambre4_1}

„Zum anderen wurde mir insbesondere auch bei Betrachtung der unterschiedlichen Bewertungssysteme in Deutschland und Frankreich während der dritten Etappe klar, dass auch, wenn man bereits Erfahrungen mit der fremden Kultur gemacht hat und zeitweise selbst - z. B. im Rahmen eines Auslandsaufenthaltes - Teil dieser Kultur war, häufig dennoch Fragen bezüglich der Verhaltensweisen der Menschen im anderen Land offen bleiben, die nur dann geklärt werden können, wenn man bereit dazu ist, diese direkt an die fremde Kultur zu stellen. So wurde mir z. B. in der dritten Etappe bei dem bloginternen Vergleich der deutschen und französischen numerischen Bewertungsskala bewusst, dass ich - obgleich ich bereits französische Noten erhalten habe - im Grunde nur eine vage Vorstellung davon habe, was sich hinter den einzelnen französischen Noten verbirgt:

(...) [J]'aimerais bien savoir (...) ce qu'est, par exemple, la différence entre 14/20 et 15/20. De plus, je me suis toujours demandé pourquoi le maximum des points est 20 en France, car (...) on dit toujours qu'il est presque impossible d'obtenir 19/20 ou même 20/20, et ça non seulement à l'école, mais encore à l'université. Alors, comment se faitil que ces notes existent s'il est impossible de les obtenir ? Est-ce que ce n'est pas frustrant de donner toujours le meilleur de soi-même sans avoir la possibilité de recevoir le maximum des points ???

Unser französisches Quadremmitglied antwortete mir darauf wie folgt:

Pour répondre à ta question, C'est [sic] vrai qu'il est difficile d'obtenir 20 ! 20 [sic] c'est la perfection et elle est rarement atteinte! lol! Mais par exemple tu peux obtenir 20 à une interro de verbes irréguliers en Anglais ou à des interros qui ne nécessitent pas un gros effort de rédaction mais où il s'agit plus d'apprendre par coeur! Tu comprends? à [sic] la fac c'est impossible (ou quasi!!!) d'obtenir cette note car il s'agit souvent de rédiger des commentaires, des dissertations... et il y a toujours quelque chose à redire! Si tu as 12/13 de moyenne générale à la fac c'est déjà pas mal du tout! plutôt bien à mon avis!

Il n'y a pas de diférences entre 14 ou 15 ! Justeque 15 c'est un peu mieux que 14 ! logique!

lol! Que tu ais 14 ou 15, dans les deux cas tu es un bon élève! au [sic] dessus de 15 alors là tu es un très bon élève! comme [sic] quoi la frontière entre les notes ne veut pas dire grand chose!

Unter Berücksichtigung von diesem und ähnlichen Beispielen hat mir die Arbeit mit meinen Quadremmitgliedern auch gezeigt, dass man, unabhängig davon, wie sehr man sich der fremden Kultur innerlich verbunden fühlen mag, doch gedanklich im Grunde noch immer stark seiner eigenen Kultur verhaftet bleibt. 
Diese Barriere zu überwinden ermöglicht meines Erachten nach ebenfalls nur die direkte interkulturelle Interaktion, die überdies - wie obiges Beispiel verdeutlicht - auch nachdem man bereits eine gewisse Zeit im Zielland verbracht hat, eine Art Post-Enkulturation im Heimatland bewirken kann: Wenn ich in Frankreich beispielsweise mit Noten wie 12/20 nach Hause gekommen bin, so hatte ich stets das Gefühl, dass ich dringend mehr für die Universität tun müsste, um meine Leistungen zu verbessern; ein Eindruck, der auch noch nach meiner Rückkehr nach Deutschland bis zu dieser Etappe Bestand hatte. Durch den Austausch mit meinem französischen Quadremsmitglied wurde mir im Nachhinein jedoch klar, dass derartige Leistungen im Prinzip ,pas mal du tout " sind und ich mich damals keineswegs hätte schlecht fühlen müssen." 


\title{
Accompagner la formation de futurs enseignants de langue en tandem interculturel médiatisé: la sensibilisation aux fonctions du tutorat
}

\author{
Dagmar Abendroth-Timmer / Jose I. Aguilar Río (Siegen, Paris)
}

\begin{abstract}
This paper presents a theoretical reflection on the role of tutoring in distance learning (cf. Bourdet 2007). This reflection was triggered by an experimental, IC-mediated collaborative learning project (cf. Bertin et al. 2010) between Siegen and Sorbonne Nouvelle universities. ICT-mediated distance learning plus the contact with the tutors allowed students to reflect on the role of tutoring, namely its differences with traditional teacher-fronted classroom contexts (cf. Aguilar 2013). Excerpts from the qualitative and quantitative data generated by the project are used to illustrate how it allowed for the students' reflection on aspects of tutor-learner relationship, as well as on the multilingual dimension of the project.
\end{abstract}

Se presenta una reflexión teórica sobre la función del tutor en enseñanzas a distancia vía las TIC (cf. Bourdet 2007). La reflexión se basa en una experiencia piloto de aprendizaje híbrido y colaborativo (cf. Bertin et al. 2010) entre las universidades de Siegen y Sorbonne Nouvelle. Gracias a la situación de aprendizaje a distancia y al contacto con los tutores, los estudiantes reflexionaron sobre la figura del tutor, a la diferencia con situaciones pedagógicas tradicionales (cf. Aguilar 2013). Los extractos de los datos cualitativos y cuantitativos generados por el proyecto ilustran cómo éste provocó la reflexión de los participantes sobre aspectos de la relación tutor-alumno, así como sobre su dimensión plurilingüe.

\section{1 À propos du concept de «tutorat"}

Notre concept de «tutorat » se décline en trois volets: a) les rôles et fonctions du tuteur; b) le rapport du tuteur avec son propre savoir, et avec celui d'autrui; c) la conception de la formation des enseignants de langue, en vue d'une sensibilisation au statut du tuteur.

\subsection{Rôles et fonctions du tuteur}

Outre la «médiation » (Bourdet 2007), l'« accompagnement» est une fonction essentielle pour le tuteur. Elle se matérialise sur trois dimensions " technique », « disciplinaire », et " méthodologique » (Denis 2003: 28), et comporte des sousfonctions: le conseil «dans le choix adéquat d'outils de communication selon les types et les moments d'activités ", la provision de "ressources », "l'organisation ", " la négociation et la répartition des tâches ", et le "soutien affectif » (ibid.). Nissen (2009: 7-8) compare des tutorats en présentiel et à distance. L'auteur (ibid.: 10-11) observe des différences significatives entre la matérialisation 
de certains rôles en présentiel et à distance, notamment pour ce qui concerne, d'une part, le rôle social (plus prenant à distance), et, d'autre part, les rôles motivationnel, cognitif et langagier (plus importants en présentiel).

\subsection{Tutorat et rapport au(x) savoir(-faire)}

La fonction d'accompagnement est liée à l'autonomie des apprenants (cf. Barbot 2006: 42), ce qui suppose une (ré)définition du rapport des participants à une situation d'apprentissage face à des savoir(-faire). Depuis une perspective socioconstructiviste, Bertin/Narcy-Combes (2007: 446) définissent la situation d'apprentissage d'une langue comme la rencontre entre un tuteur, un apprenant et une tâche à accomplir. L'interaction entre le tuteur et l'apprenant suppose une médiation, si bien que le premier accompagne le travail du deuxième, sans lui imposer une méthode ou logique quelconques. Ainsi, parmi les rôles du tuteur, celui d'expert dans un savoir ou discipline ne figurerait pas en première ligne. Ceci remet en question la conception traditionnelle d'une relation pédagogique construite autour d'un rapport triangulaire reliant un savoir, un candidat à ce savoir et un expert (cf. Rosier 2002). L'expertise du tuteur semble être d'un autre ordre. Elle porterait sur la capacité de «créer et faciliter des situations de coopération entre étudiants de culture différente » (cf. Barbot 2006: 36), complétée par un savoir-faire en évaluation (cf. Bertin/Narcy-Combes 2007: 447). Cette expertise requiert des compétences pluridisciplinaires.

\subsection{La formation au tutorat}

Selon Denis (2003: 28), les «multiples facettes » du tuteur à distance, nécessitent une formation rassemblant «psychologie sociale, méthodologie, psychologie de l'apprentissage et informatique ». D'ailleurs, cette formation doit comporter une expérience réelle du quotidien institutionnel du futur tuteur, telles le " vécu d'un dispositif de FAD en tant qu'apprenant » (ibid.: 32), afin d'avoir un « ancrage dans la pratique », de « donner du sens à la fonction tutorale » (ibid.: 33). Les multiples facettes propres à la fonction du tuteur, l'approche socioconstructiviste du savoir et le caractère pratique de la formation, autant de principes auxquels nous avons adhéré lors de la conception du projet SiegenParis, qui fait l'objet de cet article, mais aussi en tant que tuteurs intervenant dans le dispositif dont il est question dans cet article.

\subsection{Le dispositif de formation}

Le projet Siegen-Paris a été conçu par Dagmar Abendroth-Timmer, Jose Aguilar, et Jean-Paul Narcy-Combes, et tutoré par les deux premiers. Il s'agit d'une formation hybride (cf. Bertin et al. 2010) hébergée sur la plate-forme 
Moodle de l'Université de Siegen, et proposée de manière conjointe par les universités de Siegen en Allemagne et Sorbonne Nouvelle - Paris 3 en France. 11 étudiants à Paris et 10 à Siegen y ont participé. Certains étudiants avaient l'espagnol, le grec, le persan, le russe et le turc comme langue maternelle. Tous les participants avaient un projet professionnel autour de l'enseignement des langues - français langue étrangère, 'FLE', pour les Français, Lehramt pour les Allemands.

Trois vidéoconférences ont eu lieu. Au cours de la première, les étudiants ont découvert le fonctionnement de la plate-forme Moodle, les tandems ont été organisés, le calendrier a été négocié. Chaque tandem avait cinq tâches à soumettre (cf. Narcy-Combes 2005: 166-167). Pour les trois premières tâches, chaque tandem devait produire une synthèse théorique à partir de 2-3 articles choisis dans un corpus d'une trentaine d'articles scientifiques, en allemand, anglais, espagnol et français, sur la recherche en acquisition des langues, la didactique des langues et les TICE. La quatrième tâche consistait en l'analyse d'un site internet. La dernière tâche proposait une réflexion sur les apports de la formation a) au niveau de leur réflexivité individuelle et en tandem, b) au développement des compétences professionnelles en contexte multimédia et plurilingue (cf. Abendroth-Timmer 2011). Les tandems ont géré leur travail à distance grâce aux forums et au chat que permet Moodle, ainsi que via Skype. Tous les échanges ont été soumis aux tuteurs via Moodle. Chaque tandem a décidé de la langue de travail - pour la rédaction des synthèses et la gestion des échanges -, de la fréquence et de la modalité des rencontres en ligne.

Notre analyse des données portera sur les manières dont les étudiants se sont présentés lors des interactions, leurs modes de communication, et la façon dont ils ont interagi avec l'environnement plurilingue et interculturel du dispositif. Nous relierons ces observations avec la question de la construction d'une identité professionnelle. Nous examinerons ce que les étudiants affirment avoir tiré de la formation pour ce qui concerne leur sensibilisation au métier d'enseignant de langue. Nous nous focaliserons sur les notions de tutorat, plurilinguisme et d'interculturel, telles qu'elles ont été catégorisées par les étudiants.

\section{2 Épistémologie et méthodes de recherche}

La conception de l'apprentissage à la base du projet Siegen-Paris relève de principes socioculturels et socioconstructivistes (cf. Furstenberg et al. 2001; Lantolf 2007). Quant à la formation de futurs enseignants, nous avons adhéré à l'idée selon laquelle le développement d'une identité professionelle enseignante nécessite d'une prise en main dans des conditions réalistes. Notre hypothèse était que l'expérience pratique dans notre projet pourrait promouvoir un apprentissage guidé, en autonomie et autogéré. L'utilisation des TICE a été 
conçue comme un moyen permettant aux étudiants de rencontrer des interlocuteurs-informateurs faisant partie d'une réalité culturelle, géographique et institutionnelle différente de la propre. L'union en tandems de deux participants avait pour but de déclencher un processus conjoint de réflexion sur l'apprentissage des langues, la formation des enseignants de langue, les multiples appartenances, et les multilittératies revendiquées par les étudiants. Il était attendu que le travail en tandem mène vers une réflexion formative et transformatrice.

Le projet Siegen-Paris a requis la révision des rôles traditionnellement attribués à l'enseignant (cf. Aguilar 2010): tuteur a prévalu sur enseignant, le tutorat a été retenu au dépens de l'enseignement (cf. Bertin/Narcy-Combes 2007). Dagmar Abendroth-Timmer et Jose Aguilar, tuteurs, ont supervisé les tandems, assuré une médiation entre ceux-ci et la technologie, et encadré une situation d'apprentissage, dont les aspects cognitifs ont été pris en charge par les étudiants. Les tuteurs n'ont agi ni comme des détenteurs ni comme des pourvoyeurs du savoir. Ils ont gardé certains droits institutionnels dans la mesure où ils ont suivi et évalué le travail des tandems.

Afin d'illustrer les attitudes que le projet a déclenchées chez les participants, nous présentons des extraits de données tirés de quatre tandems. Ceux-ci sont représentés de manière synthétique dans le tableau ci-dessous - les prénoms des étudiants ont été anonymisés:

Tableau 1: Les tandems d'étudiants (noms anonymisés)

\begin{tabular}{|c|l|l|l|l|} 
Tandem & \multicolumn{1}{|c|}{ A } & \multicolumn{1}{|c|}{ B } & \multicolumn{1}{|c|}{ D } \\
\hline Siegen & $\begin{array}{l}\text { Béline } \\
\text { (germanophone native) }\end{array}$ & $\begin{array}{l}\text { Panthea } \\
\text { (hellenophone native) }\end{array}$ & $\begin{array}{l}\text { Edith } \\
\text { (germanophone native) }\end{array}$ & $\begin{array}{l}\text { Leonore } \\
\text { (germanophone native) }\end{array}$ \\
\hline Paris & $\begin{array}{l}\text { Olivia } \\
\text { (francophone native) }\end{array}$ & $\begin{array}{l}\text { Ludivine } \\
\text { (francophone native) }\end{array}$ & $\begin{array}{l}\text { Lydia } \\
\text { (francophone native) }\end{array}$ & $\begin{array}{l}\text { Vera } \\
\text { (russophone native) }\end{array}$ \\
\hline
\end{tabular}

Ces choix se justifient par des critères de représentativité, fréquence dans la participation, et appréciation institutionnelle de la participation des étudiants. Outre le genre - 20 étudiantes et un étudiant -4 des 21 étudiants avaient une langue maternelle différente de l'allemand et du français. Le tandem A a cumulé le plus d'échanges (1,5 Méga octets de données écrites), tandis que le tandem B est celui qui a échangé le moins (584 Kilo octets de données écrites), les tandems $\mathrm{C}$ et $\mathrm{D}$ se situant dans autour de 750 Kilo octets de données écrites environ. Enfin, l'évaluation a varié entre 19/20 (1.0/1) (Tandem A) et 14/20 (2.3/2-) (Tandem B) - 16/20 pour le tandem C (1.7/2+) et 18/20 (1.3/1-) pour le D. Les critères d'évaluation ont porté sur: a) le respect des consignes (nombre de pages, d'articles employés); b) la qualité de l'écriture académique (citations, réfé- 
rences, style, forme, orthographe, cohérence et cohésion); c) la qualité des synthèses (capacité à mobiliser des idées et notions, à synthétiser une pensée complexe, à faire des transferts vers une vie professionnelle éventuelle).

\section{Analyse qualitative: «Internet nous a permis d'être plus autonome dans notre travail ${ }^{1}$. " (tandem $\left.\mathrm{A}, \mathrm{S} 5^{2}: 35^{3}\right)$}

\subsection{Les modes d'interaction médiatisés:}

Ici il s'agissait de réfléchir avec une inconnue qui habitait à plusieurs centaines de bornes sur un sujet qui était assez vague pour l'une comme pour l'autre. (tandem B, S 5: 7)

Après la vidéoconférence d'ouverture ( $\$ 1.3)$, les étudiants ont eu le choix de communiquer via Moodle ou Skype. Certains étudiants ont mentionné leur habitude de communiquer au quotidien grâce à des outils web (Lydia, tandem C, postQ: 85), ce qui limiterait les rencontres personnelles (Edith, tandem C, postQ: 85). La différence du dispositif par rapport à des situations pédagogiques traditionnelles (Ludivine, tandem B, S 5: 10), ainsi que sa dimension plurilingue ont demandé un travail d'adaptation, comme le signale Ludivine à propos de la difficulté additionnelle pour les participants germanophones:

$[\ldots]$ déjà que c'est tout nouveau pour nous, j'imagine que pour vous c'est encore plus compliqué puisque vous communiquez en français! (Ludivine, tandem B, forum 1:35)

Dès la première vidéoconférence, le français est apparu comme la langue de communication. Cette décision s'explique du fait que tous les participants à Siegen parlaient couramment le français. Certains étudiants ont caractérisé leur méthode de travail selon un principe de « respect pour l'autre », matérialisé dans des efforts de clarté et ponctualité (Edith, tandem C, postQ 86, Lydia/Edith, tandem C, S 5: 5). Ceci semble expliquer le choix de certains tandems de prendre des décisions via Skype, réservant donc Moodle pour l'échange de textes, ou encore d'utiliser des outils d'écriture collaborative synchrone, comme Google Drive. À ce propos, Olivia remarque que la communication écrite «force à prendre en compte le point de vue de l'autre car il est écrit (on ne peut pas faire semblant de ne pas avoir entendu ou couper la parole)»(Olivia, postQ: 87). L'on distingue une communication orale gérée par les tandems - Skype - et des

\footnotetext{
${ }^{1}$ Nous rendons les propos des étudiants selon l'orthographe originale et sans aucune correction.

${ }^{2}$ Nous utilisons les abréviations suivantes: S 5 = tâches 5 en forme de synthèse ou rapport réflexif du tandem, postQ $=$ post-questionnaire.

${ }^{3}$ Numérotation des paragraphes indiquée par le logiciel Maxqda utilisé pour l'analyse qualitative.
} 
communications orales institutionnelles, organisées et gérées par les tuteurs visioconférences. Selon certains étudiants, comparée avec la communication écrite sur Moodle, la visioconférence permettait de "prendre conscience de la dimension de groupe dans l'expérience » (Olivia, postQ: 91). Certains étudiants affirment que les visioconférences n'ont pas eu d'influence sur le travail des tandems (Edith, tandem C, postQ: 91). Quant aux communications orales gérées par les tandems, les tuteurs ont donné la consigne de garder des traces écrites des échanges, à publier sur des forums Moodle prévus à cet effet.

Pour chacune des 5 tâches, un forum a été assigné à chaque tandem. L'accès et la participation dans les forums est restée ouverte. Certains étudiants ont exprimé leur regret de ne pas avoir un forum commun:

[I]l aurait fallu un forum général et un vrai chat sur moodle. idéalement il faudrait que tout passe par la même plateforme: forum, mails, chat, etc, visio, avec possibilité de passer en mode privé. (Olivia, postQ: 82)

D'autres étudiants ont signalé que c'est grâce à l'usage différencié des médias, en fonction des besoins et préférences de communication de chaque tandem, qu'une interaction fructueuse et un travail en autonomie ont été possibles:

Durch die gleichzeitige Benutzung vieler Medien konnte ein konstanter und fruchtbarer

Charakter des Austausches gewährt werden. (Béline, postQ: 81)

Le choix et l'offre de médias semblent avoir déterminé la manière de communiquer de chaque tandem. Ce sont les étudiants qui ont défini leur(s) rôle(s) dans le projet, leur degré d'investissement et d'appropriation du dispositif.

\subsection{Le rôle des participants dans l'interaction}

[L]a relation enseignant/apprenant dans le contexte multimédial est beaucoup plus sereine et encourageante que dans le contexte d'enseignement traditionnel. (tandem B, S 5: 17)

Certains étudiants ont catégorisé les tuteurs comme des "accompagnateurs » (Olivia, tandem A, forum 2: 15). D'autres ont trouvé qu'ils «étaient à la fois les concepteurs du dispositif de formation, les tuteurs et les évaluateurs des étudiants » (tandem A, S 5: 25). Ludivine insiste sur l'équilibre dans la relation entre les tuteurs - qu'elle catégorise comme "professeurs » - et les apprenants pour ce qui concerne l'imprévisibilité du projet:

Peut être qu'un cours d'utilisation du forum nous aurait fait gagner du temps. Mais le fait que les professeurs soient autant dans l'incertitude que nous étaient vraiment drôle. (Ludivine, tandem B, postQ: 100)

Pour d'autres étudiants, la fonction des tuteurs portait moins sur l'intervention que sur la motivation et l'orientation ( $§ 1.1)$, si bien que la gestion était assurée par les étudiants. Ainsi, pour Olivia et Béline: 
La majorité des interactions avec l'enseignant gommait la dimension autorité-sanction dévolue habituellement à l'enseignant, puisqu'elles consistaient essentiellement à du guidage et des encouragements. Par ailleurs, l'aspect ludique de l'expérience, faisait des enseignants des animateurs de jeu (d'où les lapsus d'Olivia tutoyant l'enseignant). Mais les séances réelles (en groupe et en [vidéoconférence]) rétablissaient le rapport traditionnel à l'enseignant. (tandem A, S 5:28)

Certains étudiants ont souligné des fluctuations dans la relation entre les tuteurs et les apprenants. Ceci serait dû à la forme respective des différents cadres d'interaction: des tuteurs dans Moodle pour réorienter, corriger, aider, des enseignants dans le contact en présentiel ou par courriel individuel lors de l'évaluation des travaux rendus (tandem A, S 5: 25). D'après certains étudiants, afin de gérer une autonomie imprégnée d'incertitudes, et d'être ouverts à la transparence des échanges en tandems sur des forums accessibles par tous les participants sur la plate-forme Moodle «il a[urait] fallu instaurer un climat de confiance [au sein du tandem] comme le faisaient à un niveau au-dessus nos professeurs $[\ldots]$ » (tandem B, S 5: 8). Cette transparence a entraîné le besoin d'un espace privé (§3.1).

Quant à l'évaluation des tâches, les étudiants allemands demandaient plus de corrections langagières puisque le développement langagier représentait pour eux un objectif explicitement nommé dans leurs pré-questionnaires (Leonore, postQ: 105). Quant aux participants à Paris, ils comprenaient que ceci ne relevait pas des objectifs primordiaux du projet:

[Vera]: ils critiquent pas severement, ils conseillent, ils nous soutiennent, l'accent est mis sur le savoir de l'apprenant qu'il acquiere a la fin et moins sur les fautes. (...) tu l'as remarque? (...) enfin pour moi, les commentaires de nos tuteurs sont comme ca. (Vera, tandem $\mathrm{D}$, forum $3: 85$ )

Ludivine (tandem B, forum 1: 73) a caractérisé l'approche des tuteurs comme " réactif», ce qui suggère que les tuteurs ont laissé du temps aux étudiants de trouver des démarches et des solutions eux-mêmes avant d'intervenir - ce qu'ils ont seulement fait lorsqu'ils ont été directement interpellés, ou bien s'ils ont détecté des risques de fausse route. D'autres étudiants qualifient la présence des tuteurs comme constante (tandem C, S 5: 8). Les tuteurs ont guidé le travail des tandems, mais ils ont aussi établi le groupe entier en tant qu'entité discursive et sociale:

Ce qui a été le rôle de José et Dagmar, indispensable selon nous au bon développement de ces échanges. Dans ce contexte, le professeur apparaît bien comme un lien humain et social, mais a aussi le rôle essentiel d'équilibrer la conversation. Cette conversation requiert un équilibre au niveau du temps de parole, mais également au niveau de la prise de parole; les échanges ayant été principalement virtuels (Skype par écrit, chat sur Moddle, mails sur Moodle), il n'est pas très naturel de prendre la parole devant un groupe d'élèves étrangers, dont parfois on découvre le visage même après avoir travaillé plusieurs semaine en binôme. Ainsi le rôle de l'enseignant apparaît comme essentiel 
afin de mener à bien les échanges et l'organisation du travail, il (ou ils) est l'élément de liaison entre les deux groupes. (tandem C, S 5:13)

Enfin, nous reprenons les propos de Lydia et Edith, qui synthétisent les fonctions du tuteur au niveau théorique, mais aussi telles que les deux tuteurs du projet les ont matérialisées:

D'après Bertin, Narcy-Combes et Gravé, la question essentielle qui sous-tend la question du tutorat en ligne est de savoir comment il est envisageable d'aider l'apprenant dans un dispositif d'apprentissage à distance afin de répondre, au moins partiellement, à plusieurs objectifs. Tout d'abord le but est de réduire le nombre d'abandons. En l'occurrence, cet objectif ne s'applique pas tellement à notre expérience, compte tenu de sa courte durée et de la motivation (expérience volontaire) de chacun. Le deuxième objectif nous concerne plus, puisqu'il s'agit d'apporter des réponses rapides aux questions de l'apprenant, de réduire le délai entre l'activité et le feedback et d'améliorer les conditions de la rétroaction didactique. Cela a été particulièrement ressenti lors de cette expérience, grâce à l'outil de la plateforme Moodle. En effet, les enseignants ont été très présents lors de nos échanges sur les forums, en tandem ou ouverts, ainsi que par mails, pour nous conseiller et nos orienter dans nos démarches, ce qui souligne un autre objectif qui est d'offrir un suivi et un étayage personnalisés, afin de répondre aux besoins de tutorat de chaque apprenant. Un quatrième objectif est de faciliter l'apprentissage en attirant l'attention de l'apprenant sur les processus cognitifs mis en œuvre dans les activités et ses stratégies d'apprentissage. En ce qui nous concerne, Dagmar et José nous ont bien précisé que cette expérience nous permettrait de construire des savoirs théoriques (avec le travail sur les textes) et pratiques (avec les échanges et la création de fiches pédagogiques sur des sites). De plus, à chaque visioconférence de groupe, nous avons pu discuter de ce que chaque tâche nous avait apporté, ayant ainsi une approche métacognitive. Enfin, un travail en tutorat à distance doit développer l'autonomie de l'apprenant $[\ldots]$ ce que nous avons pu constater clairement lors de la mise au point en tandem sur la répartition des tâches et du déroulement du travail. (tandem C, S 5: 8)

Au cours de leur participation dans le projet, les étudiants ont agi à tour de rôle en tant qu'experts des langues ou des médias. Comme les connaissances langagières étaient distribuées inégalement (§3.1), les étudiants à Paris «étai[en]t considérée[s] comme [les] «spécialiste[s] » de la langue » (tandem B, S 5: 8). Ceci étant dit, les concepteurs ont proposé des textes en plusieurs langues, afin de permettre une négociation consensuelle, selon les compétences dans chaque tandem: «Ludivine: tu seras peut être plus à l'aise avec un texte en allemand ou en anglais? » (Ludivine, tandem $\mathrm{B}$, forum 1: 61). Ce choix visait en effet à réduire les inégalités éventuelles d'expertise de nature langagière.

Il transparaît des commentaires des étudiants que le projet comportait une véritable dimension plurilingue et transnationale. Outre les faits que plusieurs nationalités étaient représentées par les étudiants-participants, et que le corpus de textes scientifiques proposés comportaient des articles originaux rédigés en plusieurs langues européennes, les propres pratiques que les participants ont matérialisées ont montré leur conscience d'habiter un espace où plus d'une 
langue était légitime afin d'accomplir les différentes étapes menant vers les tâches demandées.

\subsection{La communication plurilingue et interculturelle}

[C]e projet a exigé de nous de communiquer à volonté, de coopérer, de comprendre et se faire comprendre. (tandem B, S 5: 5)

Le projet «a offert la possibilité de lire des textes en langues différentes (anglais, français, allemand)» (tandem C, S 5: 3). Afin de gérer ce plurilinguisme, les étudiants ont utilisé plusieurs stratégies: a) répartir les textes selon les compétences langagières natives ou aussi bien acquises: «te fatigues pas avec l'article anglais. je le ferai » (Leonore, tandem $\mathrm{D}$, forum 1: 77); b) rédiger les synthèses dans une langue bien maîtrisée (Olivia, postQ: 95); c) se procurer des traductions des textes au lieu de les lire: « je lis l'allemand laborieusement mais je triche avec google-traduction » (Olivia, tandem A, forum 2: 49). En préparation à la vidéoconférence finale, certains étudiants se sont renseignés sur les contenus des textes dans une toute autre langue grâce à la lecture des synthèses des autres tandems:

par exemple, aucune de nous ne parle l'espagnol mais nous avons pu nous appuyer sur la synthèse 3 de [deux autres étudiantes] [...]. (tandem A, S 5: 51)

Quant au choix des langues pour la communication, Olivia signale que Béline " était confrontée à des situations authentiques qui ont éveillé [son] besoin de s'exprimer dans la langue étrangère. » (tandem A, S 5: 33). Comme le signalent certains étudiants, l'on retrouve chez certains tandems des alternances codiques matérialisées dans des phrases de salutation, ce qui suggère des stratégies de compréhension mutuelle:

Nous avons choisi de parler français et très rarement l'anglais ou l'espagnol venaient se greffer lorsqu'il y avait une mauvaise compréhension. (Ludivine, postQ: 89)

Cette ouverture au plurilinguisme a été également incitée par le fait que les étudiants avaient le choix des textes pour la rédaction de leurs synthèses, ainsi que de la langue de communication lors des échanges via Skype:

Grâce au choix des langues nous pouvions choisir les langues ave[c]s lesquelles nous voulions communiquer. Ce choix des langues nous a aidé d'augmenter notre compétence de plurilinguisme. (Panthea, tandem B, postQ: 89)

D'autres étudiants, non francophones ni germanophones natifs, ont regretté que leurs langues maternelles n'aient pas été prises en compte:

[P]our moi le choix de langues ne posait aucun problème: on a choisi le français comme langue de conversation et je lisais les articles en espagnol et ma partenaire lisait en allemand et en anglais. J'aurais préféré égelement la présence de ma langue maternelle car je me sentais défavorisée par rapport aux autres. (Vera, postQ: 86) 
Ceci joue un rôle important quand la langue de travail (le français) n'est pas la langue maternelle pour aucune des deux partenaires dans un tandem:

Die Wahl der Sprache ist uns nicht schwer gefallen (französisch) und hat unsere Kommunikation auch nicht negativ beeinflusst. Allerdings ist Französisch für uns beide Fremdsprache und vielleicht haben sich unsere Texte (Synthesen) dadurch doch deutlicher von denen der anderen tandems unterschieden. (Leonore, tandem D, postQ: 89)

Certains étudiants ont constaté que la situation communicative était « réelle » et authentique pour ce qui concerne la langue et la culture (tandem A, S 5: 33). D'autres étudiants ont signalé un temps insuffisant pour discuter de questions extérieures au projet - notamment de nature interculturelle:

Wir haben uns (fast) nicht über Persönliches ausgetauscht und somit auch nicht Themen wie z.B. unsere Kenntnisse von Frankreich bzw. Deutschland angesprochen. (Edith, tandem C, postQ: 83)

Toutefois, certains étudiants signalent comment l'échange sur des contenus académiques a parfois conduit vers une réflexion interculturelle:

[I]1 est vrai que correspondre en français avec une personne dont la langue maternelle n'est pas le français et qui n'a jamais mis les pieds en France demande parfois de clarifier certains point culturels et socio-linguistiques. Que se soit intra ou extra synthèse nous avons, à plusieurs reprises eu l'occasion de discuter voir de débattre sur des questions interculturelles. (Ludivine, tandem B, postQ: 83)

D'après certains étudiants, il aurait été préférable, premièrement, de mettre plus explicitement en avant l'interculturel:

Und ich hätte eventuell Nebenaufgaben gestellt in Bezug auf interkulturelle Kompetenzen. So hätte man einen Anstoss für den interkulturellen Austausch geschaffen. Die Aufgaben hätten auch weniger umfangreich sein können, so wäre mehr Zeit für private Kommunikation geblieben. (Leonore, postQ: 100)

Deuxièmement, il aurait fallu intégrer l'interculturel davantage dans les tâches. En dehors de cette suggestion des étudiants, on constate chez certains étudiants une conscience vraisemblablement claire de ce qu'est l'interculturel:

Ich denke, dass diese Art von Kommunikation zur Entwicklung von interkulturellen Kompetenzen beitragen kann. Der Austausch über Kultur, über universitäre Strukturen, über Kenntnisse vom jeweils anderen Land o.ä. findet allerdings nicht unbedingt statt, es sei denn, diese Themen werden explizit in den Aufgabestellungen angesprochen. Die Arbeitsweise des Tandempartners lernt man bei der gemeinsamen Arbeit auf jeden Fall kennen. $\mathrm{Ob}$ diese Arbeitsweise allerdings typisch für das jeweils andere Land ist, ist meines Erachtens nach zu hinterfragen. Ich würde nicht von der Arbeitsweise einer Person, auf die charakteristische Arbeitsweise z. B. der Franzosen schließen. (Edith, tandem C, postQ: 84)

L'on retrouve cette conscience plus concrètement centrée sur l'aspect interculturel dans un projet franco-allemand dans un autre propos:

[I]l nous semble que l'interculturel est une disposition d'esprit envers l'autre et qu'elle ne se limite pas aux nationalités mais concerne toute rencontre, enfin parce que nous 
sommes déjà familières de ce type de situation et que l'interculturel France - Allemagne ne nous semble pas représenter un défi exceptionnel pour la compréhension mutuelle. (tandem A, S 5: 9)

L'apparente réussite du projet quant à la prise de conscience des défis plurilingues, culturels et pédagogiques de l'apprentissage, se manifeste dans ce récit. Ceci fait la transition de l'expérience universitaire à la réalité scolaire.

\subsection{Développer l'identité professionnelle}

[C] e projet nous a fait travailler sur les TICE et par les TICE. (tandem B, S 5: 12)

Dans leurs rapports réflexifs et dans les post-questionnaires les étudiants ont fait la liaison entre leur propre expérience et leur future vie professionnelle. Les étudiants se sont auto-catégorisés comme futurs enseignants et ont montré leur capacité d'analyser leur expérience sur ce plan professionnel futur. Les sections précédentes ont montré la manière profonde d'analyser aussi bien la fonction des tuteurs dans un tel projet que les moyens de communication. Lorsque les étudiants s'attaquent à la tâche de faire le transfert de la formation vers une réalité scolaire prospective, ils affirment que cette expérience leur a permis d'intégrer la théorie et la pratique:

En rétrospective, la conception paraît toute à fait logique. Nous avons pu apprendre les notions théoriques tout en voyant leur réalisation en pratique dans le cadre de notre formation. (tandem D, S 5: 9)

Ce projet expérimental et concret aussi bien au niveau de l'usage de différents médias qu'au niveau plurilingue et interculturel a donné aux étudiants des idées pratiques pour leur future vie professionnelle:

En effet, On peut choisir d'orienter les apprenants vers un auto-apprentissage guidé, de les accompagner dans un échange interculturel avec d'autres pays voir les deux en même temps! (tandem B, S 5: 15)

Pour conclure, certains étudiants affirment comment leur participation a contribué à ce qu'ils s'investissent davantage dans un travail académique de réflexion théorique:

L'aspect ludique et moins académique que l'enseignement traditionnel m'a donné l'envie de réaliser mon mémoire de fin d'étude sur ce thème! (Ludivine, tandem $\mathrm{B}$, postQ: 106)

\section{Conclusions}

Les extraits montrés suggèrent que les étudiants ayant participé au projet Siegen-Paris ont été sensibles aux objectifs méta-pédagogiques de formation interculturelle et plurilingue des concepteurs, d'autant qu'ils ont observé dans 
les actions des tuteurs des modèles pédagogiques pertinents et éventuellement intégrables lors de leurs expériences enseignantes futures, d'après leurs avis.

L'usage des TIC ne semble pas avoir été négativement perçu par les participants. D'une part, certains étudiants ont manifesté leurs habitudes d'internaute, ce qui semble les avoir préparés à utiliser des technologies déjà connues dans un cadre institutionnel et formatif. D'autre part, nous observons un investissement de la part des participants, si bien qu'ils donnent des indices d'appropriation du dispositif, dans la mesure où ils ont remarqué des manques, auxquels ils ont proposé, voire trouvé, des solutions techniques adéquates et adaptées à leurs besoins ponctuels. Ainsi, nous observons une attitude chez certains étudiants qui suggère que la dimension virtuelle présente dans le projet ne comportait pas une dimension d'irréalité - bien que cette dimension virtuelle limite l'expérience du réel. L'irréel correspondrait plutôt au contrat de fiction qui semble s'établir entre un enseignant et un groupe d'apprenants, observant un ordre institutionnel où serait appliquée une pédagogie traditionnelle, asymétrique, inégalitaire et peu favorable à l'autonomie des apprenants. La notion du tutorat que les deux tuteurs du dispositif se sont efforcés de transmettre par leurs actions semble avoir atteint une partie des étudiants - dans la mesure où ceux-ci affirment avoir fait une différence entre la manière de faire des tuteurs et celle d'enseignants dans un cadre traditionnel. Or, il demeure que, lors de la tâche finale, une grande partie des étudiants a catégorisé les tuteurs comme « enseignants ». Ceci est l'indice de quelque chose qui pourrait bien ressembler à de la résistance. La relation asymétrique propre d'une pédagogie traditionnelle semble ancrée dans les représentations de certains des étudiants participants. Par contre, il faut également prendre en considération le fait que les étudiants connaissaient au moins un des deux tuteurs également dans un rôle plus traditionnel dans le cadre de cours en présentiel de leurs universités.

Enfin, il est impossible de déterminer l'impact que la participation à une formation comme celle-ci peut avoir sur une pratique future, tant que l'on ne se donne pas les moyens de l'observer. Afin de corriger cette insuffisance, nous avons mis au point, en collaboration avec Lutz Küster, Jean-Paul Narcy-Combes et Katharina Wieland, une deuxième version élargie du projet Siegen-Paris. La première partie de cette nouvelle expérience reproduit celle que nous avons décrite ici. Au cours de la deuxième partie, les étudiants-participants seront mis en situation de concepteurs, afin de se rapprocher davantage des conditions professionnelles réelles d'un tuteur intervenant dans un environnement d'apprentissage de langues. 


\section{Bibliographie}

Abendroth-Timmer, Dagmar (2011). Reflexive Lehrerbildung: Konzepte und Perspektiven für den Einsatz von Unterrichtssimulation und Videographie. Zeitschrift für Fremdsprachenforschung 18: 3-41.

Aguilar Río, Jose I. (2010). Pour une analyse de la "présentation de soi » de l'enseignant de L2: Style revendiqué, aspects relationnels, décisions interactionnelles. Thèse de doctorat non publiée. Sorbonne Nouvelle - Paris III, Paris.

Aguilar Río, Jose I. (2013). The Institutional and Beyond: On the Identity Displays of Foreign Language Teachers. In: Rivers, Damien / Houghton, Stéphanie (éds.): Social Identities and Multiple Selves in Foreign Language Education. London et New York: Bloomsbury. 1331.

Barbot, Marie-Jo (2006). Rôle de l'enseignant-formateur: l'accompagnement en question. Mélanges CRAPEL 28: 29-46.

Bertin, Jean-Claude / Narcy-Combes, Jean-Paul (2007). Monitoring the Learner - Who, Why and What For? Computer Assisted Language Learning 20/5: 443-457.

Bertin, Jean-Claude / Gravé, Patrick / Narcy-Combes, Jean-Paul (2010). Second Language Distance Learning and Teaching: Theoretical Perspectives and Didactic Ergonomics. Hershey: IGI Global.

Bourdet, Jean-François (2007). Tutorat en ligne et création d'un espace formatif. ALSIC 10/1: 23-32.

Denis, Brigitte (2003). Quels rôles et quelle formation pour les tuteurs intervenant dans des dispositifs de formation à distance? Distances et savoirs 1/1: 19-46.

Furstenberg, Gilbert / Levet, Sabine / English, Kathryn / Maillet, Katherine (2001). Giving a Virtual Voice to the Silent Language of Culture: The Cultura Project. Language Learning \& Technology 5/1: 55-102.

Lantolf, James P. (2007). Sociocultural source of thinking and its relevance for second language acquisition. Bilingualism. Language and Cognition 10/1: 31-33.

Narcy-Combes, Jean-Paul (2005). Didactique des langues et TIC: vers une recherche-action responsable. Paris: Ophrys.

Nissen, Elke (2009). Quels rôles le tuteur joue-t-il en distantiel et en présentiel? Analyse des interactions verbales d'une formation hybride. In: Develotte, Christine / Mangenot, François / Nissen, Elke (coord.): Actes du colloque Epal 2009 (Echanger pour apprendre en ligne: conception, instrumentation, interactions, multimodalité), Université Stendhal Grenoble 3, 5-7 juin 2009.

Rosier, Jean-Maurice (2002). La didactique du français. Paris: PUF. 



\section{Authors}

Prof. Dr. Dagmar Abendroth-

Timmer

Universität Siegen

Fakultät 1: Philosophische Fakultät

Didaktik der spanischen und

französischen Sprache und Kultur

Adolf-Reichwein-Str. 2

57068 Siegen

Germany

abendroth@romanistik.uni-siegen.de

Jose I. Aguilar Río, maître de conférences

Université Paris III

Sorbonne Nouvelle

DFLE

46, rue St Jacques

75230 Paris cedex 5

France

Jose.aguilarrio@univ-paris3.fr

Prof. em. Dr. Gerhard Bach

FB 10 - Universität Bremen

Bibliothekstraße 1

28359 Bremen

gbach@uni-bremen.de

Jun.-Prof. Dr. Mark Bechtel

Technische Universität Dresden

Institut für Romanistik

Didaktik der romanischen Sprachen

Zeunerstr. 1c

01062 Dresden

Germany

mark.bechtel@mailbox.tu-

dresden.de
Prof. Dr. Stephan Breidbach

Humboldt-Universität zu Berlin

Philosophische Fakultät II

Institut für Anglistik und

Amerikanistik

Fachdidaktik Englisch / Foreign

Language Education

Unter den Linden 6

10099 Berlin

Germany

stephan.breidbach@hu-berlin.de

Assistant Professor Alice Chik

City University of Hong Kong

Department of English

M8100

Level 8

Run Run Shaw Creative Media

Centre

18 Tat Hong Avenue, Kowloon

Tong, Hong Kong

China

Alice.chik@cityu.edu.hk

Maud Ciekanski, maître de conférences

Université Paris 8

Laboratoire EXPERICE (Paris 8Paris 13)

2, rue de la Liberté

93526 Saint-Denis cedex

France

maud.ciekanski@univ-fcomte.fr 
Eva de Mesmaeker

Vrije Universiteit Brussel

Multi-L

Pleinlaan 2

1050 Brussels

Belgium

evdemesm@vub.ac.be

Prof. Dr. Sabine Ehrhart

Université du Luxembourg

Campus Walferdange

7220 Walferdange

Luxemburg

sabine.ehrhart@uni.lu

Prof. Dr. Daniela Elsner

Goethe-Universität Frankfurt

Didaktik und Sprachlehrforschung

IEAS

Grüneburgplatz 1

60323 Frankfurt/Main

Germany

elsner@em.uni-frankfurt.de

Prof. Dr. Olga Esteve

Universitat Pompeu Fabra

Roc Boronat

13808018 Barcelona

Spain

olga.esteve@upf.edu

Assistant Professor Özlem Etuş

İstanbul University

English Language Teaching

Department

Ebulula Mardin Cad. Park Maya Sit.

Carlton: 6, D: 14, Akatlar, Beşiktaş,

Turkey; oziletus@istanbul.edu.tr
Prof. Dr. Christiane Fäcke

Universität Augsburg

Universitätsstr. 10

86159 Augsburg

Germany

christiane.faecke@phil.uni-

augsburg.de

Dr. Claudia Frevel

Universität Siegen

Fakultät I: Philosophische Fakultät

Didaktik der spanischen und französischen Sprache und Kultur Adolf-Reichwein-Str. 2 57068 Siegen

Germany

claudia.frevel@uni-siegen.de

Prof. Dr. Claus Gnutzmann

Senator-Bauer-Str. 23,

30625 Hannover

Germany

c.gnutzmann@tu-braunschweig.de

Eva-Maria Hennig

Universität Siegen

Fakultät I: Philosophische Fakultät

Didaktik der spanischen und

französischen Sprache und Kultur

Adolf-Reichwein-Str. 2

57068 Siegen

Germany

hennig@romanistik.uni-siegen.de

Dipl.-Päd. Mahzad Hoodgarzadeh

RKW Niedersachsen; Germany

mhoodgarzadeh@hotmail.de 
Jenny Jakisch

TU Braunschweig

Englisches Seminar, Abt. Englische

Sprache und ihre Didaktik

Bienroder Weg 80

38106 Braunschweig

Germany

j.jakisch@tu-braunschweig.de

Prof. Dr. Lutz Küster

Humboldt-Universität zu Berlin

Philosophische Fakultät II:

Institut für Romanistik

Didaktik der romanischen Sprachen

und Literaturen

Unter den Linden 6

10099 Berlin

Germany

lutz.kuester@romanistik.hu-

berlin.de

Prof. Dr. Katja Lochtmann

Vrije Universiteit Brussel

Multi-L

Pleinlaan 2

1050 Brussels

Belgium

katja.lochtman@vub.ac.be

Professeurs des Universités

Jean-Paul Narcy-Combes

(Université Sorbonne nouvelle;

DILTEC) \&

Marie-Françoise Narcy-Combes

(Université de Nantes; CRINI)

2, rue du Commandant Rivière

44000 Nantes - France

jean-paul.narcy-combes@univparis3.fr

mf.narcycombes@wanadoo.fr
Professor Anne Pauwels

University of London

SOAS, Faculty of Languages and

Cultures

Thornhaugh Street

Russell Square

London WC1H 0XG

England

ap62@soas.ac.uk

Professor Martha C. Pennington

English Department

City University of Hong Kong

Run Run Shaw

Creative Media Centre

Cornwall Street

Kowloon Tong, Kowloon

Hong Kong

China

mcpenn@cityu.edu.hk

Frank Rabe

Technische Universität

Braunschweig

Englisches Seminar

Bienroder Weg 80

38106 Braunschweig

Germany

f.rabe@tu-bs.de

Professor Itesh Sachdev

University of London

SOAS

Department of Linguistics

Thornhaugh Street

London WC1H 0XG

United Kingdom

i.sachdev@soas.ac.uk 
Katrin Schultze

Humboldt-Universität zu Berlin

Institut für Anglistik und

Amerikanistik

Fachdidaktik Englisch / Foreign

Language Education

Unter den Linden 6

10099 Berlin

Germany

katrin.schultze@hu-berlin.de

Prof. Dr. Britta Viebrock

Goethe-Universität Frankfurt

Didaktik und Sprachlehrforschung

IEAS

Grüneburgplatz 1

60323 Frankfurt/Main

Germany

viebrock@em.uni-frankfurt.de

Rebecca Walter

Universitat Pompeu Fabra

Roc Boronat

13808018 Barcelona

Spain

rebecca.walter@upf.edu

Martina Weyreter

Goethe-Universität Frankfurt

Institut für Psychologie

Arbeitsbereich Pädagogische

Psychologie

Grüneburgplatz 1

60323 Frankfurt/Main

Germany

weyreter@paed.psych.uni-

frankfurt.de
Prof. Dr. Anja Wildemann

Universität Koblenz-Landau

Institut für Bildung im Kindes- und

Jugendalter

August-Croissant-Straße 5

76829 Landau

Germany

wildemann@uni-landau.de 


\title{
Kolloquium Fremdsprachenunterricht
}

\author{
Herausgegeben von Daniela Caspari, Lars Schmelter,
} Karin Vogt und Nicola Würffel

Kolloquium Fremdsprachenunterricht (KFU) publiziert Tagungsdokumentationen und thematisch ausgerichtete Sammelbände sowie einschlägige Monographien, Dissertationen und Habilitationsschriften zu allen relevanten Fragenstellungen der Fremdsprachenforschung. Insbesondere folgende Forschungsgebiete sind von Interesse: Fremdsprachendidaktik aller Sprachen, Fremdsprachenlehr- und -lernforschung, Forschungsmethoden, Mehrsprachigkeitsforschung, interkultureller Fremdsprachenunterricht, Multiliteralitätsforschung, bilingualer Unterricht, mediengestütztes Fremdsprachenlernen und -lehren, Literatur- und Textdidaktik.

Publikationsanfragen richten Sie bitte an eine/n der HerausgeberInnen, Prof. Dr. Daniela Caspari, Prof. Dr. Lars Schmelter, Prof. Dr. Karin Vogt, Prof. Dr. Nicola Würffel. Gerne berät das Herausgeberteam interessierte Autorlnnen bei Forschungsprojekten.

Prof. Dr. Daniela Caspari: http://www.geisteswissenschaften.fu-berlin.de/we05/mitarbeiter/caspari/ index.html

Prof. Dr. Lars Schmelter: http://www.romanistik.uni-wuppertal.de/personal/fachdidaktik/prof-dr-phillars-schmelter.html

Prof. Dr. Karin Vogt: http://www.ph-heidelberg.de/englisch/personen/lehrende/prof-dr-vogt.html

Prof. Dr. Nicola Würffel: http://www.ph-heidelberg.de/mediendidaktik/personen/lehrende/prof-dr-nicolawuerffel.html

Band 1 Volker Raddatz / Michael Wendt (Hrsg.): Textarbeit im Fremdsprachenunterricht - Schrift, Film, Video. Kolloquium zur Ehren von Bertolt Brandt (Verlag Dr. Kovac 1997).

Band 2 Gabriele Blell/Wilfried Gienow (Hrsg.): Interaktion mit Texten, Bildern, Multimedia im Fremdsprachenunterricht (Verlag Dr. Kovac 1998).

Band 3 Renate Fery / Volker Raddatz (Hrsg.): Lehrwerke und ihre Alternativen. 2000.

Band 4 Gisèle Holtzer/Michael Wendt (éds.): Didactique comparée des langues et études terminologiques. Interculturel - Stratégies - Conscience langagière. 2000.

Band 5 Gerhard Bach/Susanne Niemeier (Hrsg.): Bilingualer Unterricht. Grundlagen, Methoden, Praxis, Perspektiven. 5., überarbeitete und erweiterte Auflage. 2010.

Band 6 Michael Wendt (Hrsg.): Konstruktion statt Instruktion. Neue Zugänge zu Sprache und Kultur im Fremdsprachenunterricht. 2000.

Band 7 Dagmar Abendroth-Timmer/Stephan Breidbach (Hrsg.): Handlungsorientierung und Mehrsprachigkeit. Fremd- und mehrsprachliches Handeln in interkulturellen Kontexten. 2000.

Band 8 Wolfgang Zydatiß: Leistungsentwicklung und Sprachstandserhebungen im Englischunterricht. Methoden und Ergebnisse der Evaluierung eines Schulversuchs zur Begabtenförderung: Gymnasiale Regel- und Expressklassen im Vergleich. Unter Mitarbeit von Viola Vockrodt-Scholz. 2002.

Band 9 Wilma Melde/Volker Raddatz (Hrsg.): Innovationen im Fremdsprachenunterricht 1. Offene Formen und Frühbeginn. 2002.

Band 10 Gerhard Bach / Britta Viebrock (Hrsg.): Die Aneignung fremder Sprachen. Perspektiven Konzepte - Forschungsprogramm. 2002.

Band 11 Hannelore Küpers / Marc Souchon (Eds.): Appropriation des Langues au Centre de la Recherche. Spracherwerb als Forschungsgegenstand. 2002. 
Band 12 Helene Decke-Cornill / Maike Reichart-Wallrabenstein (Hrsg.): Fremdsprachenunterricht in medialen Lernumgebungen. 2002.

Band 13 Nikola Mayer: Ganzheitlichkeit und Sprache. Theorie des Begriffs und empirische Zugangswege im Gespräch mit Fremdsprachenlehrerinnen- und -lehrern. 2002.

Band 14 Brigitte Krück/Christiane Loeser (Hrsg.): Innovationen im Fremdsprachenunterricht 2. Fremdsprachen als Arbeitssprachen. 2002.

Band 15 Johannes Eckerth / Michael Wendt (Hrsg.): Interkulturelles und transkulturelles Lernen im Fremdsprachenunterricht. 2003.

Band 16 Dagmar Abendroth-Timmer/Britta Viebrock/Michael Wendt (Hrsg.): Text, Kontext und Fremdsprachenunterricht. Festschrift für Gerhard Bach zum 60. Geburtstag. 2003.

Band 17 Petra Bosenius/Jürgen Donnerstag (Hrsg.): Interaktive Medien und Fremdsprachenlernen. 2004.

Band 18 Mercedes Díez / Raquel Fernández / Ana Halbach (eds.): Debate en torno a las estrategias de aprendizaje. Debating Learning Strategies. 2004.

Band 19 Adelheid Schumann (Hrsg.): Kulturwissenschaften und Fremdsprachendidaktik im Dialog. Perspektiven eines interkulturellen Französischunterrichts. 2005.

Band 20 Christine Penman (ed.): Holistic Approaches to Language Learning. 2005.

Band 21 Steffi Morkötter: Language Awareness und Mehrsprachigkeit. 2005.

Band 22 Wolfgang Zydatiß: Bildungsstandards und Kompetenzniveaus im Englischunterricht. Konzepte, Empirie, Kritik und Konsequenzen. 2005.

Band 23 Gerhard Bach/Gisèle Holtzer (éds.): Pourquoi apprendre des langues? Orientations pragmatiques et pédagogiques. 2006.

Band 24 Andreas Grünewald: Multimedia im Fremdsprachenunterricht. Motivationsverlauf und Selbsteinschätzung des Lernfortschritts im computergestützten Spanischunterricht. 2006.

Band 25 Christiane Fäcke: Transkulturalität und fremdsprachliche Literatur. Eine empirische Studie zu mentalen Prozessen von primär mono- oder bikulturell sozialisierten Jugendlichen. 2006.

Band 26 Laurence Jeannot: Introduction des TICE en contexte scolaire et autonomie dans l'apprentissage des langues étrangères. Stratégies et compétence d'apprentissage dans des dispositifs mixtes en France et en Allemagne. 2006.

Band 27 Helmut Johannes Vollmer (Hrsg.): Synergieeffekte in der Fremdsprachenforschung. Empirische Zugänge, Probleme, Ergebnisse. 2007.

Band 28 Jean E. Conacher/Helen Kelly-Holmes (eds.): New Learning Environments for Language Learning. Moving beyond the classroom? 2007.

Band 29 Daniela Caspari/Wolfgang Hallet/Anke Wegner/Wolfgang Zydatiß (Hrsg.): Bilingualer Unterricht macht Schule. Beiträge aus der Praxisforschung. 2., durchgesehene Auflage. 2009.

Band 30 Sabine Doff/Torben Schmidt (Hrsg.): Fremdsprachenforschung heute. Interdisziplinäre Impulse, Methoden und Perspektiven. 2007.

Band 31 Daniela Elsner/Lutz Küster/Britta Viebrock (Hrsg.): Fremdsprachenkompetenzen für ein wachsendes Europa. Das Leitziel „Multiliteralität“. 2007.

Band 32 Adelheid Schumann / Lieselotte Steinbrügge (Hrsg.): Didaktische Transformation und Konstruktion. Zum Verhältnis von Fachwissenschaft und Fremdsprachendidaktik. 2008. 
Band 33 Dagmar Abendroth-Timmer: Akzeptanz und Motivation. Empirische Ansätze zur Erforschung des unterrichtlichen Einsatzes von bilingualen und mehrsprachigen Modulen. 2007.

Band 34 Christine Hélot / Britta Benert / Sabine Ehrhart / Andrea Young (éds.): Penser le bilinguisme autrement. 2008.

Band 35 Christiane Fäcke (Hrsg.): Sprachbegegnung und Sprachkontakt in europäischer Dimension. 2009.

Band 36 Dagmar Abendroth-Timmer/Daniela Elsner/Christiane Lütge/Britta Viebrock (Hrsg.): Handlungsorientierung im Fokus. Impulse und Perspektiven für den Fremdsprachenunterricht im 21. Jahrhundert. 2009.

Band 37 Karin Aguado/Karen Schramm/Helmut Johannes Vollmer (Hrsg.): Fremdsprachliches Handeln beobachten, messen, evaluieren. Neue methodische Ansätze der Kompetenzforschung und der Videographie. 2010.

Band 38 Bernd Tesch: Kompetenzorientierte Lernaufgaben im Fremdsprachenunterricht. Konzeptionelle Grundlagen und eine rekonstruktive Fallstudie zur Unterrrichtspraxis (Französisch). 2010.

Band 39 Maria Giovanna Tassinari: Autonomes Fremdsprachenlernen. Komponenten, Kompetenzen, Strategien. 2010.

Band 40 Daniela Caspari / Lutz Küster (Hrsg.): Wege zu interkultureller Kompetenz. Fremdsprachendidaktische Aspekte der Text- und Medienarbeit. 2010.

Band 41 Dagmar Abendroth-Timmer / Marcus Bär/Bàrbara Roviró / Ursula Vences (Hrsg.): Kompetenzen beim Lernen und Lehren des Spanischen. Empirie und Methodik. 2011.

Band 42 Daniela Elsner/Anja Wildemann (Hrsg./eds.): Sprachen lernen - Sprachen lehren. Language Learning - Language Teaching. Perspektiven für die Lehrerausbildung in Europa. Prospects for Teacher Education across Europe. 2011.

Band 43 Paula Krüger: Sprachlernhabitus und Bedeutungskonstruktion beim Fremdsprachenlernen. 2011.

Band 44 Lena C. Bellingrodt: ePortfolios im Fremdsprachenunterricht. Empirische Studien zur Förderung autonomen Lernens. 2011.

Band 45 Urška Grum: Mündliche Sprachkompetenzen deutschsprachiger Lerner des Englischen. Entwicklung eines Kompetenzmodells zur Leistungsheterogenität. 2012.

Band 46 Susanne Staschen-Dielmann: Narrative Kompetenz im bilingualen Geschichtsunterricht. Didaktische Ansätze zur Förderung der schriftlichen Diskursfähigkeit. 2012.

Band 47 Corinna Koch: Metaphern im Fremdsprachenunterricht: Englisch, Französisch, Spanisch. 2013.

Band 48 Karin Aguado / Lena Heine / Karen Schramm (Hrsg.): Introspektive Verfahren und Qualitative Inhaltsanalyse in der Fremdsprachenforschung. 2013.

Band 49 Erscheint in Kürze.

Band 50 Dagmar Abendroth-Timmer / Eva-Maria Hennig (eds.): Plurilingualism and Multiliteracies. International Research on Identity Construction in Language Education. 2014.

www.peterlang.com 
